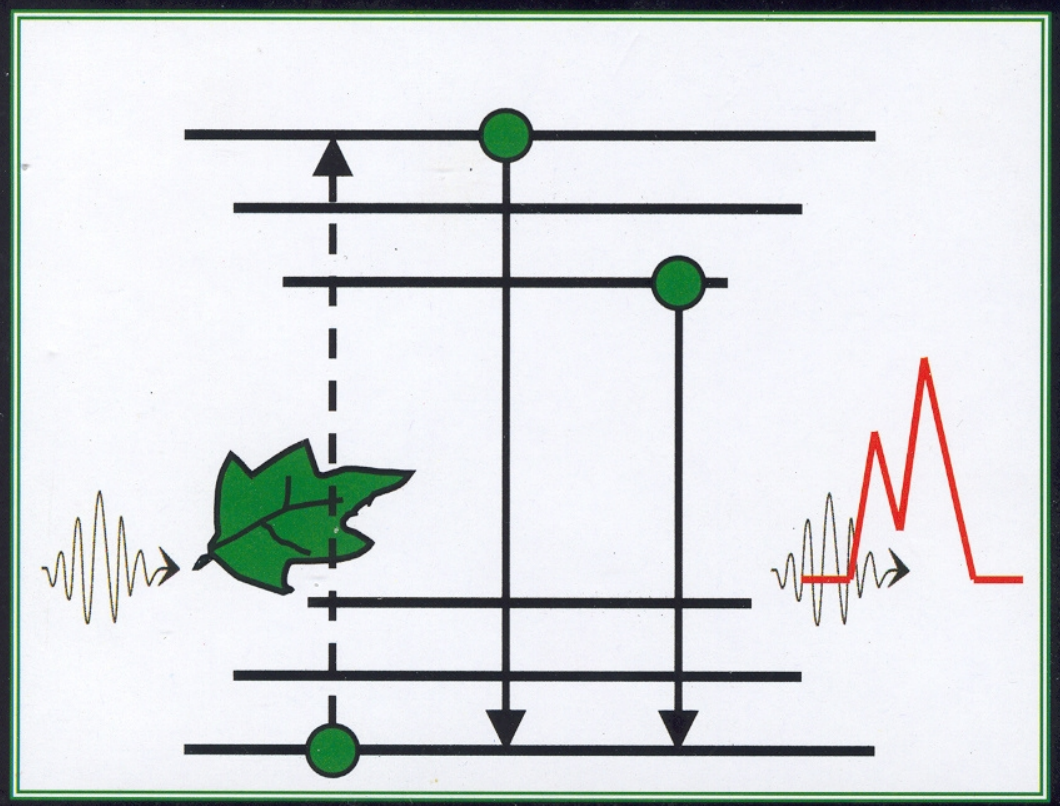

Practical Spectroscopy in Agriculture and Food Science 


\section{Practical Spectroscopy in Agriculture and Food Science}




\title{
Practical Spectroscopy in Agriculture and Food Science
}

\author{
Yuriy I. Posudin \\ National Agricultural University, \\ Kiev, Ukraine
}

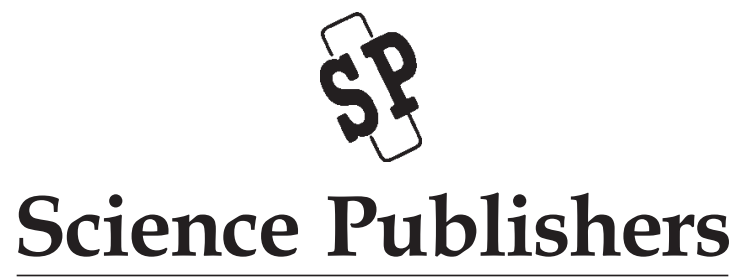

Enfield (NH) Jersey Plymouth 
CIP data will be provided on request.

SCIENCE PUBLISHERS

An imprint of Edenbridge Ltd., British Isles.

Post Office Box 699

Enfield, New Hampshire 03748

United States of America

Website: $h t t p: / / w w w . s c i p u b . n e t$

sales@scipub.net (marketing department)

editor@scipub.net (editorial department)

info@scipub.net (for all other enquiries)

ISBN 1-57808-505-5

978-1-57808-505-7

(C) 2006, Copyright reserved

All rights reserved. No part of this publication may be reproduced, stored in a retrieval system, or transmitted in any form or by any means, electronic, mechanical, photocopying or otherwise, without the prior permission.

This book is sold subject to the condition that it shall not, by way of trade or otherwise be lent, re-sold, hired out, or otherwise circulated without the publisher's prior consent in any form of binding or cover other than that in which it is published and without a similar condition including this condition being imposed on the subsequent purchaser.

Published by Science Publishers, Enfield, NH, USA

An imprint of Edenbridge Ltd.

Printed in India 


\section{Preface}

The book Practical Spectroscopy in Agriculture and Food Science introduces the students and specialists of agricultural and food science to the fundamentals of optical spectroscopy, main principles of modern spectroscopic instrumentation, advantages and practical applications of spectroscopic methods to investigation of agricultural objects such as milk and dairy products, eggs, honey, animal hair, and agronomic plants.

The significant achievements of several years of research activity by myself and my colleagues from The National Agricultural University of Ukraine are reflected in scientific articles which are published in Russian or Ukrainian. The correspondence with many people from various countries who expressed interest in this activity, provided the stimulus to publish a monograph in English, where the experience gained in the field of agricultural spectroscopy can be made available to a wide audience.

The principal sections of the material in this monograph were taught as lecture series by the author who visited the University of Georgia, USA, as a Fulbright scholar in 1996.

The author is grateful to his Ukrainian and Russian colleagues V.I. Kostenko, O.V. Tsyganjuk, P.P. Tsarenko, T.K. Gordienko, S.A. Ivanitskaya, B.M. Gopka, V.F. Lepeshenkov, A.L. Trofimenko, V.P. Polishuk, L.S. Chervinsky, N.I. Yasinetska, T.L. Zharkikh, A.D. Alyokhin, G.I. Gaydidey, M.G. Skrishevskaya, E.M. Galushko, N.G. Golubeva, M.M. Krolivets and V.A. Myasnikov.

Special appreciation is expressed to Professor Stanley J. Kays and Dr. G.G. Dull, University of Georgia, USA, who introduced the author to the fascinating world of non-destructive quality evaluation of agricultural products.

The author is also grateful to Professor Dr. H. Lichtenthaler for being given the opportunity to carry out the study of fluorescence analysis of plants in the Karlsruhe University, Germany. 


\section{vi Preface}

This book is intended for agricultural and food specialists, teachers, students and postgraduate students.

Yuriy I. Posudin 


\section{Contents}

Preface v v

1. Fundamentals of Spectroscopy 1

1.1 Optical Radiation 1

1.2 Interaction of Light and Agricultural Objects 2

1.3 Quantum Theory 3

1.4 Atomic Absorption and Emission 4

1.5 Molecular Absorption and Emission 4

1.6 Electronic Energy Levels $\quad 7$

1.7 Vibrational Energy Levels 8

1.8 Processes of Deactivation 8

1.9 Spectroscopy 10

$\begin{array}{ll}1.10 \text { Summary } & 10\end{array}$

1.11 References 10

2. Laser: Principles and Mechanisms of Action 12

2.1 Principle of Action 12

2.2 Pumping 12

2.3 Spontaneous Emission 12

2.4 Stimulated Emission 13

2.5 Absorption 13

2.6 Inversion of Population 14

2.7 Components of Laser 15

2.8 Formation of Laser Radiation $\quad 15$

2.9 Properties of Laser Radiation 18 
2.10 Summary 18

2.11 References 19

3. Spectroscopic Methods 20

3.1 Absorption/Transmission Spectroscopy 20

3.2 Light Reflection 22

3.3 Luminescence 24

3.4 Scattering 25

3.5 Summary 26

3.6 References 28

4. Spectroscopic Analysis of Milk and Dairy Products 29

4.1 Composition of Milk 29

4.2 Infrared Spectrophotometry of Milk and Dairy Products 30

4.3 Instrumentation 30

4.3.1 Optical Density of Milk $\quad 30$

4.3.2 Infrared Spectrophotometry of Milk and Dairy Products

4.4 Results of Infrared Spectrophotometry of Milk and Dairy Products 33

4.5 Infrared Spectrophotometer for Milk Analysis 38

4.6 Near-infrared Spectroscopy of Milk and Milk Components

4.7 Instrumentation 41

4.8 Results of Near-infrared Spectroscopy of Milk 42

4.9 Fluorescence Spectroscopy of Milk and Milk Components 47

4.10 Instrumentation $\quad 52$

4.11 Results of Fluorescence Spectroscopy of Milk 53

4.11.1 Whole Milk 53

4.11.2 Proteins 54

4.11.3 Fat Acids $\quad 56$

4.11.4 Vitamins 57

4.12 Identification of Milk Fluorophores 62

4.12.1 Selection of Excitation Wavelength 62

4.12.2 Decomposition of Fluorescence Spectra 64 
4.13 Fluorometers for Milk Analysis 66

4.13.1 Fluorometer for Determination of Protein and Fat Content in Milk 66

4.13.2 Laser-induced Fluorometry of Milk 66

4.14 Laser Light Scattering by Milk Particles 69

4.14.1 Theory of Light Scattering 69

4.14.2 Application of Theory of Light Scattering to Milk Particles $\quad 71$

4.15 Instrumentation $\quad 74$

4.16 Results of Light Scattering by Milk Particles 74

4.17 Summary $\quad 75$

$\begin{array}{ll}4.18 \text { References } & 79\end{array}$

5. Spectroscopic Analysis of Eggs $\quad 81$

5.1 Properties of Eggs 81

5.1.1 Structure and Contents of the Egg 81

5.1.2 Eggshell Colour and Pigmentation $\quad 81$

5.2 Current Problems of Egg Quality Evaluation 83

5.3 Absorption/Transmission Spectroscopy of Eggs in Visible Part of Spectrum 83

5.4 Fluorescence Spectroscopy of Eggs 84

5.5 Defectoscopy of Eggshell 85

5.6 Instrumentation 85

5.7 Results of Spectroscopic Analysis of Eggs and Eggshells 87

5.7.1 Reflectance Spectra 87

5.7.2 Transmission Spectra $\quad 87$

5.7.3 Fluorescence Spectra $\quad 88$

5.7.4 Defectoscopy of Eggshell $\quad 89$

5.8 Summary 90

5.9 References 91

6. Spectroscopic Analysis of Honey 93

6.1 Properties of Honey 93

6.1.1 Definition of Honey 93

6.1.2 Composition of Honey 93

6.2 Spectroscopic Analysis of Honey 94

6.3 Honey Samples 94 
6.4 Instrumentation $\quad 95$

6.5 Results of Spectroscopic Analysis of Honey 96

6.6 Summary 101

6.7 References 103

\section{Spectroscopic Analysis of Animal Hair and Bird} Feathering 105

7.1 Properties of Animal Hair-covering 105

7.1.1 Structure of Hair-covering 105

7.1.2 Colouration of Animal Hair-covering 106

7.2 Hair Samples 106

$\begin{array}{ll}7.3 \text { Instrumentation } & 108\end{array}$

$\begin{array}{ll}\text { 7.3.1 Spectrometer } & 108\end{array}$

7.3.2 Laser Diffractometry 110

7.4 Results of Spectroscopic Analysis of Animal Hair 111

7.4.1 Microphotometry and Microspectrophotometry of Horse Hair 111

7.4.2 Microfluorometry of Horse Hair 117

7.4.3 Laser Diffractometry of Animal Hair and Nasolabial Mirror 126

7.5 Selection in Cattle-breeding 131

7.6 Laser Control of Bird Feathering 132

$\begin{array}{ll}7.7 \text { Summary } & 136\end{array}$

$\begin{array}{ll}7.8 \text { References } & 136\end{array}$

\section{Spectroscopic Analysis of Agronomic Plants 138}

8.1 Laser Spectrofluorometry of Agronomic Plants 138

8.1.1 Photosynthesis and Fluorescence of Chlorophyll 138

8.1.2 Fluorescence Properties of Agronomic Plants 139

8.1.3 Plant Samples 140

8.2 Instrumentation 140

8.3 Results of Laser Spectrofluorometry of a Single Leaf 143

8.3.1 Fluorescence Indices 143

8.3.2 Fluorescence Emission Spectra during Greening of Etiolated Leaves 143

8.3.3 Effect of Pre-illumination 151

8.3.4 Fluorescence Emission Spectra of Vegetables 152 
8.3.5 Effect of the Leaf Side 154

8.3.6 Effect of Leaf Nodal Position and Age 161

8.3.7 Effect of Fertilization 162

8.3.8 Effect of Plant Diseases 162

8.4 Fluorescence Induction Kinetics 166

8.4.1 Theory of Fluorescence Induction Kinetics $\quad 166$

8.4.2 Plant Samples 167

8.5 Instrumentation 168

8.6 Results of Detection of Chlorophyll Fluorescence
Kinetics

8.6.1 Fluorescence Indices 169

8.6.2 Determination of Pigments 170

8.6.3 Effect of Leaf Nodal Position and Age 172

8.6.4 Development of a Leaf 174

8.6.5 Upper and Lower Leaf Sides 176

8.7 Vector Method of Fluorescence Analysis of Plants under Stress Conditions 176

8.7.1 Introduction 176

8.7.2 Principle of Vector Method 178

8.7.3 Application of Vector Method 179

8.8 Summary 184

8.9 References 186

$\begin{array}{ll}\text { Conclusion } & 189\end{array}$

$\begin{array}{ll}\text { Index } & 192\end{array}$ 


\section{Fundamentals of Spectroscopy}

\subsection{OPTICAL RADIATION}

Optical radiation (light in wide meaning of the word) includes the electromagnetic waves that have a wavelength from about $300 \mathrm{~nm}$ (ultraviolet region), through the visible region (400-700 nm, that can be perceived by human vision), and into the infrared region (from $760 \mathrm{~nm}$ to $2,500 \mathrm{~nm}$ ).

Optical radiation has a dual nature because it is found both as waves and particles. According to the classical interpretation, electromagnetic radiation consists of changing electric and magnetic fields that propagate through space forming an electromagnetic wave (Fig. 1.1). This wave is

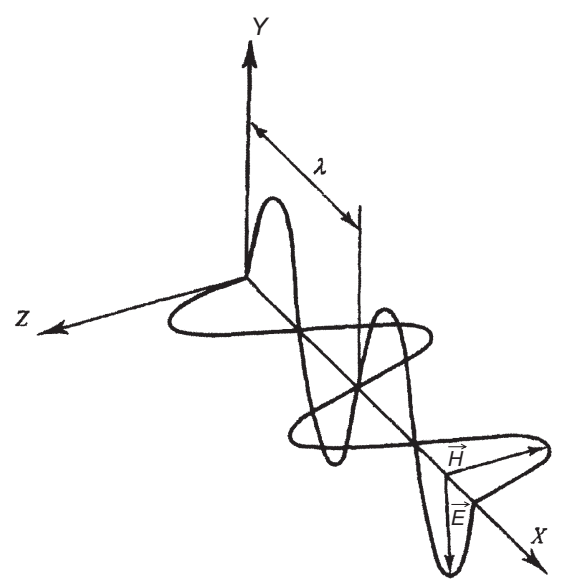

Fig. 1.1 Electromagnetic radiation consists of changing electric and magnetic fields that propagate through space forming an electromagnetic wave 
characterized by the amplitude, wavelength and polarization. In terms of the quantum theory, electromagnetic radiation consists of particles called photons - discrete packets (quanta) of electromagnetic energy that move at the speed of light. Each photon is characterized with its energy $E=h v$ and momentum $p=h / \lambda$, where $h$ is Planck's constant, $v$ is the frequency, and $\lambda$ is the wavelength of the electromagnetic wave. Dualism of light is presented by the formula $E=h v$, where the energy $E$ is related to the particle, and frequency $v$ is associated with the wave. That is why photon is depicted graphically as a wavy arrow (Fig. 1.2).

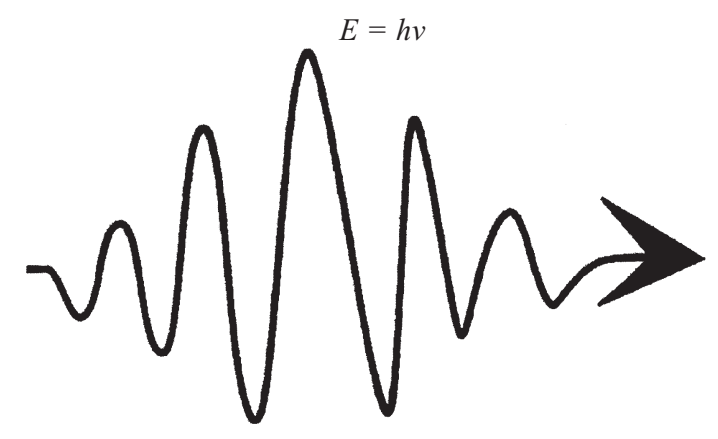

Fig. 1.2 Graphical depiction of photon as a wavy arrow

\subsection{INTERACTION OF LIGHT AND AGRICULTURAL OBJECTS}

An optical radiation that interacts with agricultural objects takes part in some processes: it can be absorbed by this object and transmitted by it, reflected by its surface, scattered by structural elements, and re-emitted by internal pigments (Fig. 1.3) [Ingle and Crouch, 1988; Analytical Instrumentation Handbook, 1997]. If we neglect the participation of scattering and re-emission, this interaction can be described by the following equation:

$$
I_{0}=I_{a}+I_{t}+I_{r}
$$

where $I_{0}$ is the total intensity of radiation incident on the object; $I_{a^{\prime}} I_{t}$, and $I_{r}$ describe the intensity of radiation absorbed, transmitted and reflected correspondingly by the object.

Dividing the equation (1.1) on $I_{0}$, it is possible to obtain the following expression:

$$
1=\frac{I_{a}}{I_{0}}+\frac{I_{t}}{I_{0}}+\frac{I_{r}}{I_{0}}=\alpha+\tau+\rho
$$




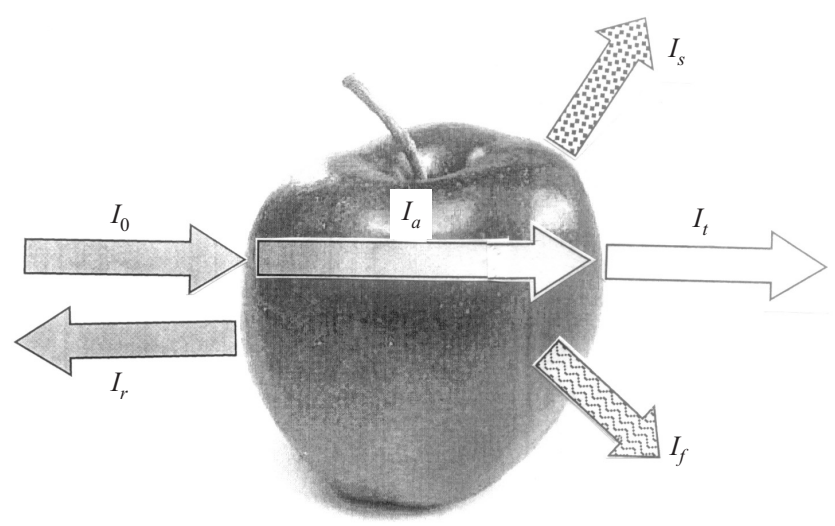

Fig. 1.3 An interaction of optical radiation $I_{0}$ with agricultural objects: absorption $I_{a}$ and transmission $I_{t}$ of it by this object, reflection $I_{r}$ by its surface, scattering $I_{s}$ by structural elements, re-emission $I_{f}$ by internal pigments

where $\alpha=\frac{I_{a}}{I_{0}}$ is the coefficient of absorption (or absorptance), $\tau=\frac{I_{t}}{I_{0}}-$ coefficient of transmission (or transmittance), and $\rho=\frac{I_{r}}{I_{0}}-$ coefficient of reflection (or reflectance).

\subsection{QUANTUM THEORY}

The principal ideas of quantum theory were developed over a period of time from 1900 till 1930. According to Planck's hypothesis, absorption and emission of light by atoms and molecules occurs not continuously but by discrete portions - quanta, the energy of which is equal:

$$
E=h v
$$

where $h=6.63 \cdot 10^{-34} \mathrm{~J} \cdot \mathrm{s}-$ Planck's constant, $v$ - frequency.

Quantum of electromagnetic radiation (in a strict sense - of light) is called photon (from Greek phos, photos - light).

In 1913, N. Bohr postulated that atoms and molecules have only certain stationary states which correspond to the discrete number of allowed values of energy $E_{i}(i=1,2,3 \ldots)$. The change of this energy is related to quantum (intermittent) transition from one stationary state to another (Fig. 1.4a); it is impossible to occupy the intermediate state between stationary states (Fig. 1.4b). 
(a)
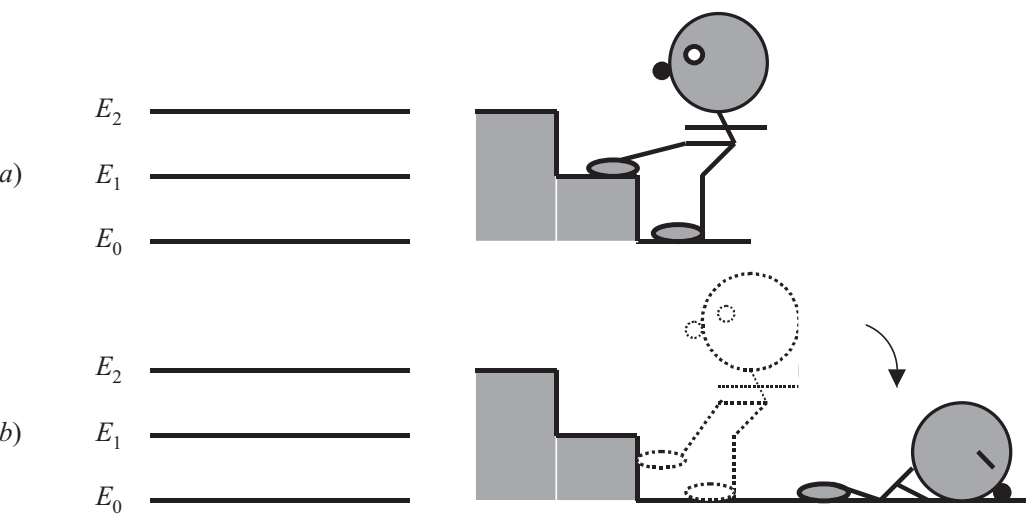

Fig. 1.4 The change of energy is related to quantum (intermittent) transition from one stationary state to another $(a)$; it is impossible to occupy the intermediate state between stationary states $(b)$

\subsection{ATOMIC ABSORPTION AND EMISSION}

Let us discuss the two-level energy structure of an atom. It consists of ground level $E_{0}$ and excited level $E_{1}$. Absorption or emission of photon takes place if the energy of photon is equal exactly to the energy difference between state with energy $E_{i}$ and state with energy $E_{j}$ :

$$
E_{i}-E_{j}=h v
$$

Absorption of photon with energy $h v=E_{1}-E_{0}$ by atom promotes it from the ground state to excited state (Fig. 1.5). Atoms are in excited state about $10^{-8} \mathrm{~s}$, and than they relax to their ground state. A downward transition is accompanied with emission of photon of energy $h v=E_{1}-E_{0}$ (Fig. 1.6). A plot of the decrease in radiant power during absorption (Fig. 1.7a) or emission (Fig. 1.7b) as a function of frequency (Fig. 1.7c) or wavelength (Fig. 1.7d) is called absorption (or emission) line.

\subsection{MOLECULAR ABSORPTION AND EMISSION}

A molecule is quite a complex system which consists of a number of atoms that participate in three types of motion: electronic (movement of electrons around nuclei), vibrational (oscillation of nuclei around the equilibrium state), and rotational (rotation of the molecule as a whole system in space). That is why the energy $E$ of a molecule is associated with the corresponding types of energy: 

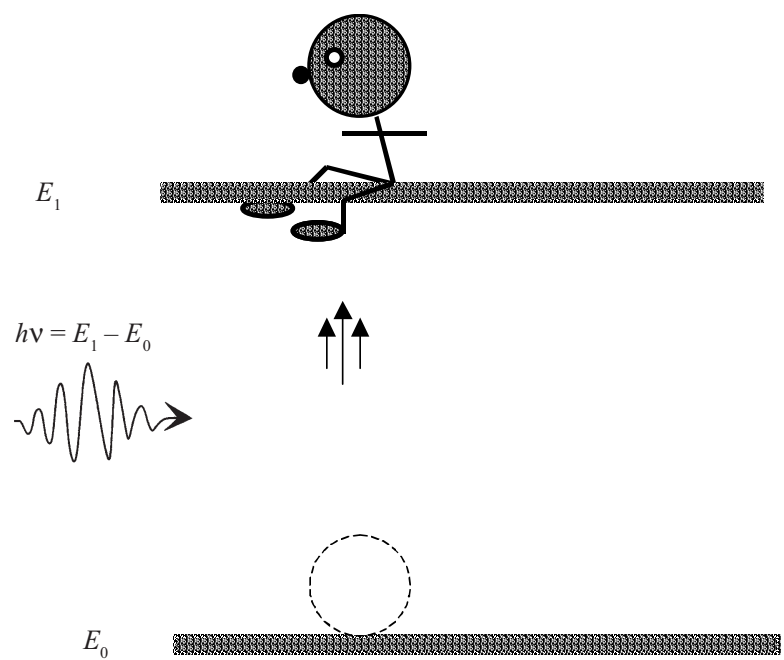

Fig. 1.5 Absorption of photon with energy $h v=E_{1}-E_{0}$ by atom promotes it from the ground state to excited state

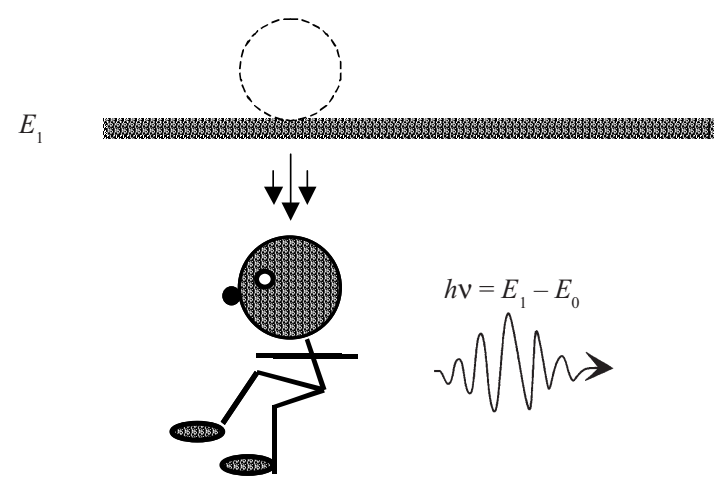

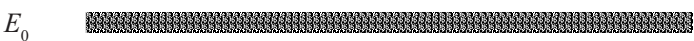

Fig. 1.6 A downward transition is accompanied with emission of photon of energy $h v=E_{1}-E_{0}$

$$
E=E_{e l}+E_{v i b r}+E_{r o t}
$$

where $E_{e l}$ is the electronic energy of molecule, $E_{v i b r}$ - the energy associated with vibrations of atoms in the molecule, $E_{\text {rot }}$ - the energy due to rotational motions of atoms within the molecule. 
6 Practical Spectroscopy in Agriculture and Food Science

(a)

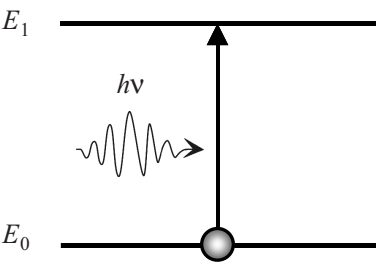

(c)

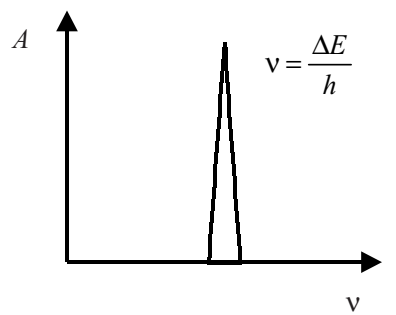

$h v=E_{1}-E_{0}=\Delta E$

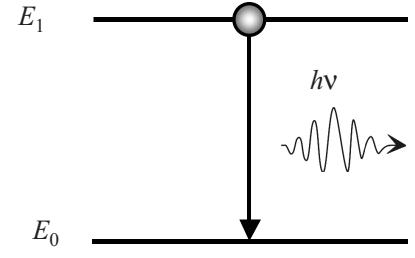

(b)

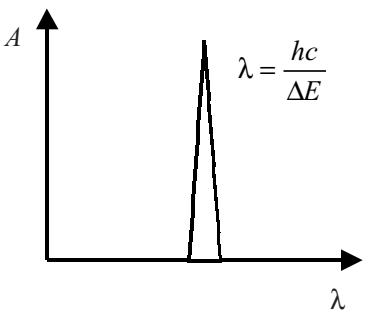

Fig. 1.7 A plot of the decrease in radiant power during absorption $(a)$ or emission $(b)$ as a function of frequency $(c)$ or wavelength $(d)$ is called absorption (or emission) line

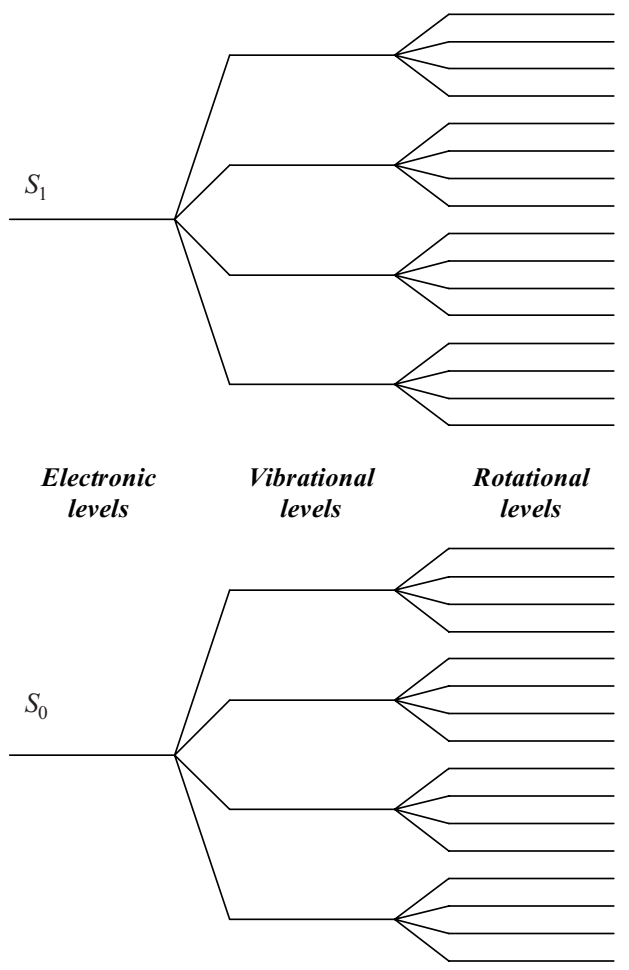

Fig. 1.8 The total energy of a molecule has certain discrete values which are characterized by a set of electronic, vibrational and rotational levels 
The total energy $E$ has certain discrete values which are characterized by a set of electronic, vibrational and rotational levels (Fig. 1.8). The transitions between these levels are accompanied by the formation of broad bands of absorption (Fig. 1.9).

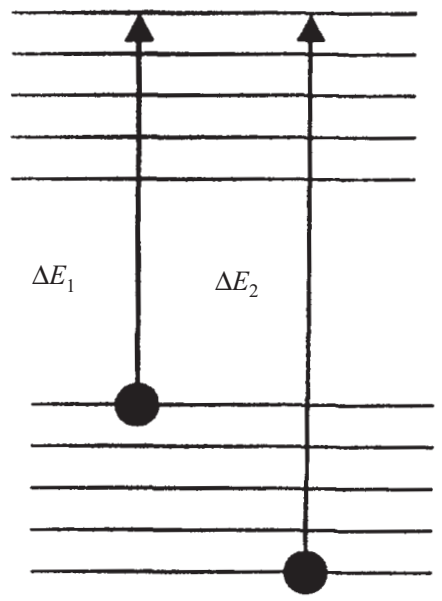

(a)

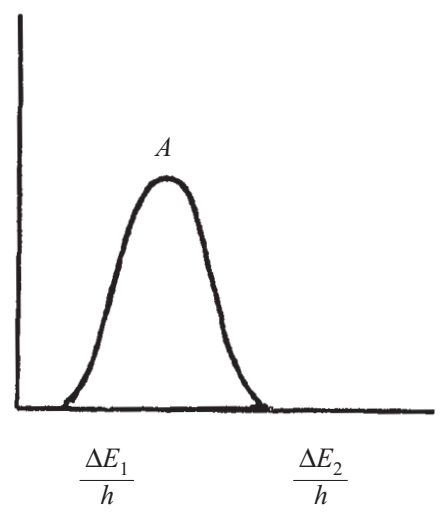

(b)

Fig. 1.9 The transitions between electronic, vibrational and rotational levels are accompanied by the formation of broad bands of absorption

The process of molecular emission is characterized by the transition of excited molecules between sublevels; the maximum of emission band is shifted to the long-wave region in comparison with the maximum of absorption band (Fig. 1.10).

\subsection{ELECTRONIC ENERGY LEVELS}

The fact is that each electron of molecule moves not only in orbital motion, but also rotates around its own axis. The energy of rotation is quantized also. The angular momentum of electron is called spin. According to the Pauli Exclusion Principle no two electrons in an atom can fit in an orbital; furthermore, the two must have opposed spin states. A molecular electronic state in which all electron spins have opposed electron spins is called a singlet state. If the two electrons are parallel, such a molecular state is called a triplet state. The average lifetime of an excited singlet state is $10^{-5}-10^{-8} \mathrm{~s}$, and that of an excited triplet state from $10^{-4} \mathrm{~s}$ to several seconds. The whole set of energy levels of the molecule is called energy level diagram (Fig. 1.11). 


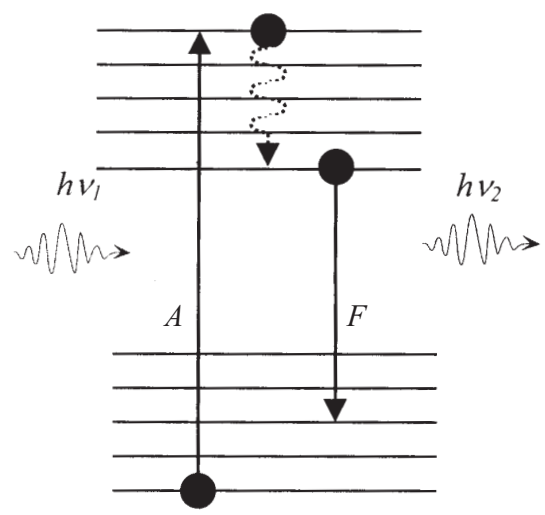

(a)

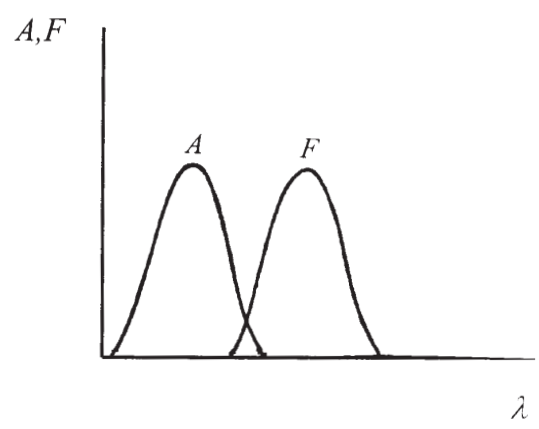

(b)

Fig. 1.10 The maximum of emission band is shifted to the long-wave region in comparison with the maximum of absorption band

\subsection{VIBRATIONAL ENERGY LEVELS}

A molecule is a flexible structure whose atoms participate in vibrational motion. Vibrational energy of such a system is quantized. The allowed energies of vibration are given by:

$$
E_{v i b r}=(v+1 / 2) h v
$$

where $v=0,1,2, \ldots-$ vibrational quantum number.

Quantum theory states the following selection rules: the only allowed vibrational transitions are those in which $\Delta v= \pm 1$. The transition from energy state 0 to 1 for any of the vibrational states $\left(v_{1}, v_{2}, v_{3}, \ldots\right)$ is considered a fundamental, if $\Delta v= \pm 1$. The transition from ground energy state to a state $v_{i}=2,3, \ldots$, is called an overtone. Transitions from ground energy state to a state $v_{i}=1$ and $v_{j}=1$ simultaneously are known as combinations [Handbook of Near-Infrared Analysis, 1992].

\subsection{PROCESSES OF DEACTIVATION}

Molecules in excited state can pass to a lower-energy state through nonradiative and radiative processes [Ingle and Crouch, 1988; Analytical Instrumentation Handbook, 1997].

Nonradiative processes involve: 


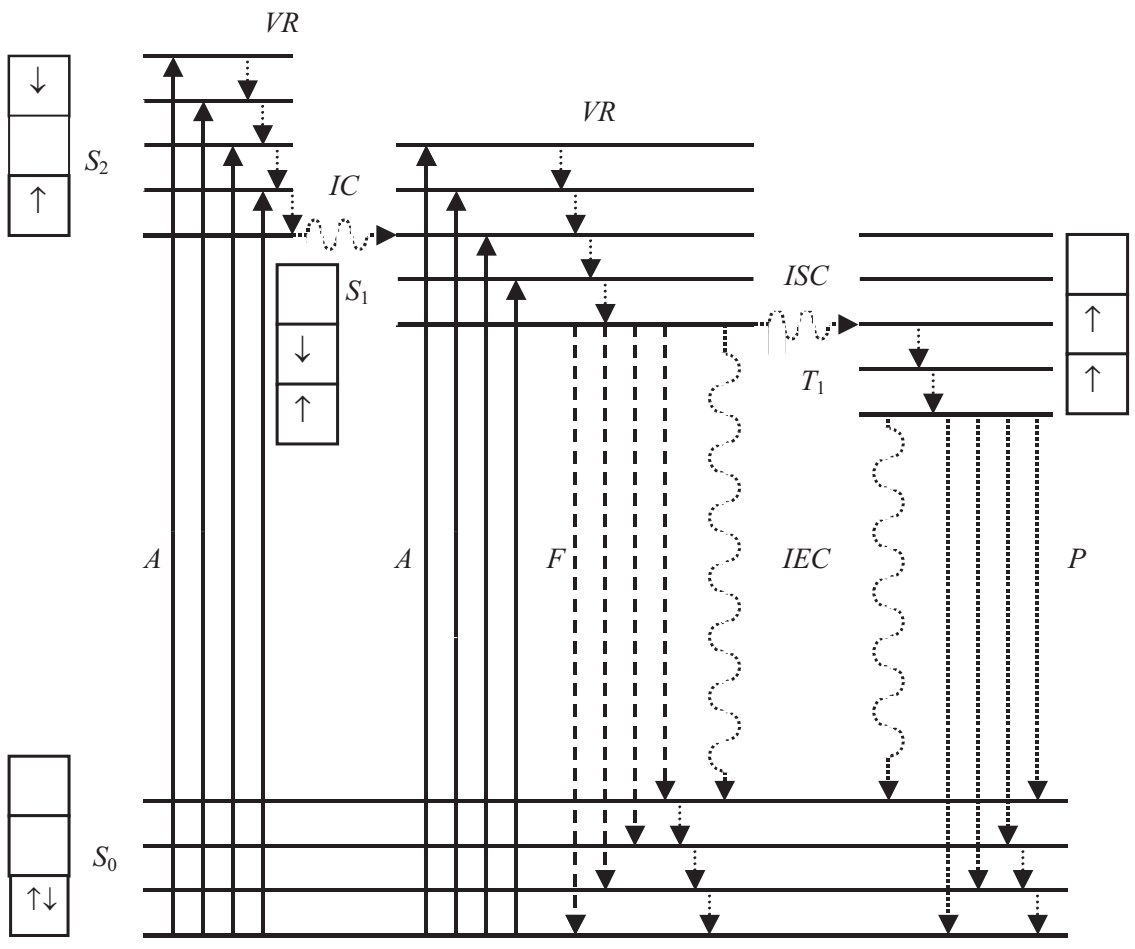

Fig. 1.11 Energy level diagram as the whole set of energy levels of molecule. $A-$ absorption, $F$ - fluorescence, $P$ - phosphorescence, VR - vibrational relaxation, IC internal conversion, ISC - intersystem crossing, IEC - internal and external conversion, $S_{0}$ - ground electronic state, $S_{1}$ - first singlet electronic state, $S_{2}$ - second singlet electronic state, $T_{1}-$ first triplet electronic state

Vibrational relaxation $V R$ accompanies the transition of molecule to the ground vibrational state in a given electronic excited state; the energy of the molecule is transferred into heat or vibrational motion. A typical period of vibrational relaxation is $10^{-14}-10^{-12} \mathrm{~s}$.

Internal conversion IC appears when lower vibrational levels of the upper electronic state overlap upper vibrational levels of lower electronic state. This process lasts for $10^{-12} \mathrm{~s}$.

Intersystem crossing ISC takes place when the vibrational states of singlet and triplet electronic excited states are overlapped.

Radiative processes involve:

Fluorescence $F$ means a radiational transition between ground vibrational state of singlet excited electronic state $S_{1}$ and various vibrational states of ground electronic state $S_{0}$ : 


$$
S_{1} \rightarrow S_{0}+h v
$$

Fluorescence requires $10^{-10} \mathrm{~s}$ to take place.

Phosphorescence $P$ refers to radiational transitions between ground vibrational state of triplet excited electronic state $T_{1}$ and various vibrational states of ground electronic state $S_{0}$ :

$$
T_{1} \rightarrow S_{0}+h v
$$

Usually, this process lasts $10^{-10}-10^{4} \mathrm{~s}$.

All these processes of deactivation are depicted in Fig. 1.11 by wave arrows (nonradiative processes) and straight vertical arrows (radiative processes).

\subsection{SPECTROSCOPY}

Spectroscopy is a scientific study of spectra of electromagnetic radiation for qualitative and quantitative analysis of the structure and properties of matter.

A spectrum is a dependence of the intensity of radiation absorbed, transmitted, reflected, scattered or re-emitted by a sample on the wavelength or frequency of electromagnetic radiation.

The following types of spectroscopic methods were used during the investigations of agricultural objects [Posudin, 1996]: Visible Absorption/ Transmission Spectroscopy; Visible Reflectance Spectroscopy; Infrared Spectrophotometry; Near-Infrared Reflectance Spectroscopy; Fluorescence Spectroscopy; Light Scattering Technique.

The main agricultural objects such as milk, eggs, honey, animal hair and agronomic plants were used for spectroscopic investigation [Posudin, 1988].

\subsection{SUMMARY}

Investigation of the dependence of the intensity of radiation absorbed, transmitted, reflected, scattered or re-emitted by an agricultural object (milk, eggs, honey, animal hair and agronomic plants) on the wavelength or frequency of optical radiation provides qualitative and quantitative analysis of the structure and properties of these objects.

\subsection{REFERENCES}

Burns D.A., Ciurczak E.W., eds. 1992. Handbook of Near-Infrared Analysis. Marcel Dekker, Inc, New York-Basel-Hong Kong. p 681. 


\section{Fundamentals of Spectroscopy $\mathbf{1 1}$}

Galen Wood Ewing, ed. 1997. Analytical Instrumentation Handbook. Marcel Dekker, Inc., New York-Basel. p 1453.

Ingle, J.D. and Crouch, S.R. 1988. Spectrochemical Analysis. Prentice Hall, Englewood Cliffs, New Jersey. p 590.

Posudin, Yu. I. 1988. Spectroscopy Monitoring of Agrosphere. Urozhaj, Kyiv. p 128.

Posudin, Yu. I. 1996. Methods of Optical and Laser Spectroscopy in Agriculture. Seminar Series of visiting Fulbright Scholar. University of Georgia, U.S.A. January-April 1996.

Posudin, Yu. I. 2005. Methods of Nondestructive Quality Evalutation of Agricultural and Food Products. Aristey, Kyiv. p 408. 


\section{Laser: Principles and Mechanisms of Action}

\subsection{PRINCIPLE OF ACTION}

Laser is a device which generates coherent electromagnetic waves due to stimulated radiation by an active medium that is placed in an optical resonator. The principle of laser action can be explained by an acronym for Light Amplification by Stimulated Emission of Radiation (LASER).

The main processes of laser action include pumping, spontaneous emission, stimulated emission and absorption [Svelto, 1976].

\subsection{PUMPING}

This is a process that provides the excitation of the active particles (atoms, molecules, ions) by means of an external source of energy. These particles are transferred from ground energy level to the higher energy levels during pumping. Some excited states are long-lived; these states are termed as metastable.

\subsection{SPONTANEOUS EMISSION}

This process is realized through the transition of excited particles from the metastable state $E_{1}$ to the ground state $E_{0}$. Such a radiative process is related to the emission of photon with the energy $h v=E_{1}-E_{0}$. It is necessary to mention that all the emitted photons have different phases and directions of propagation (Fig. 2.1). In such a way, spontaneous radiation is incoherent monochromatic radiation. 


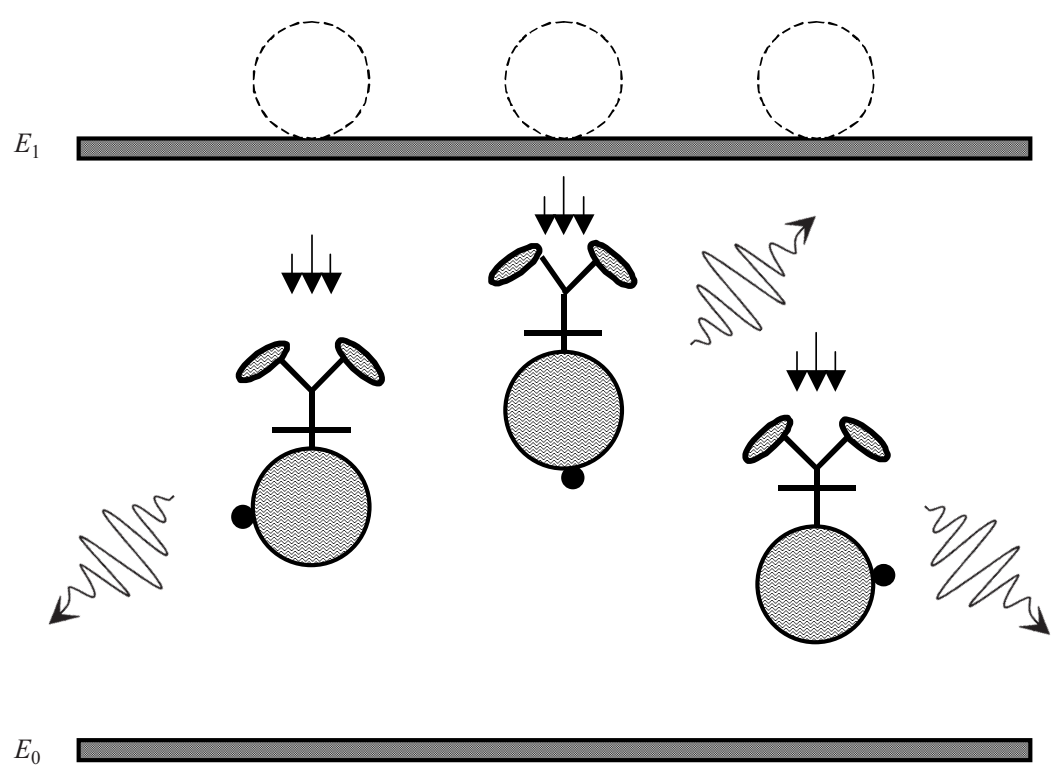

Fig. 2.1 Spontaneous radiation as incoherent monochromatic radiation which consists of the emitted photons that have different phases and directions of propagation

\subsection{STIMULATED EMISSION}

This kind of radiation takes place when an external photon with energy $h v=E_{1}-E_{0}$ strikes the excited particle and provokes the transition of these particles from the excited state to the ground state. The most important feature of this process is that emitted photons travel in exactly the same direction and are precisely in phase with the external photon which caused stimulated emission. That is why stimulated emission is coherent with the incoming radiation (Fig. 2.2). As soon as both the incoming and emitted photons travel in phase, there is an amplification of the light - a single photon stimulates the appearance of several photons (Fig. 2.3).

\subsection{ABSORPTION}

There is another process which competes with light amplification; this process is called absorption. If the external photon interacts with the laser medium, a certain number of particles are transferred from $E_{0}$ to $E_{1}$; this process is characterized by decreasing of the number of photons (Fig. 2.4). 
14 Practical Spectroscopy in Agriculture and Food Science

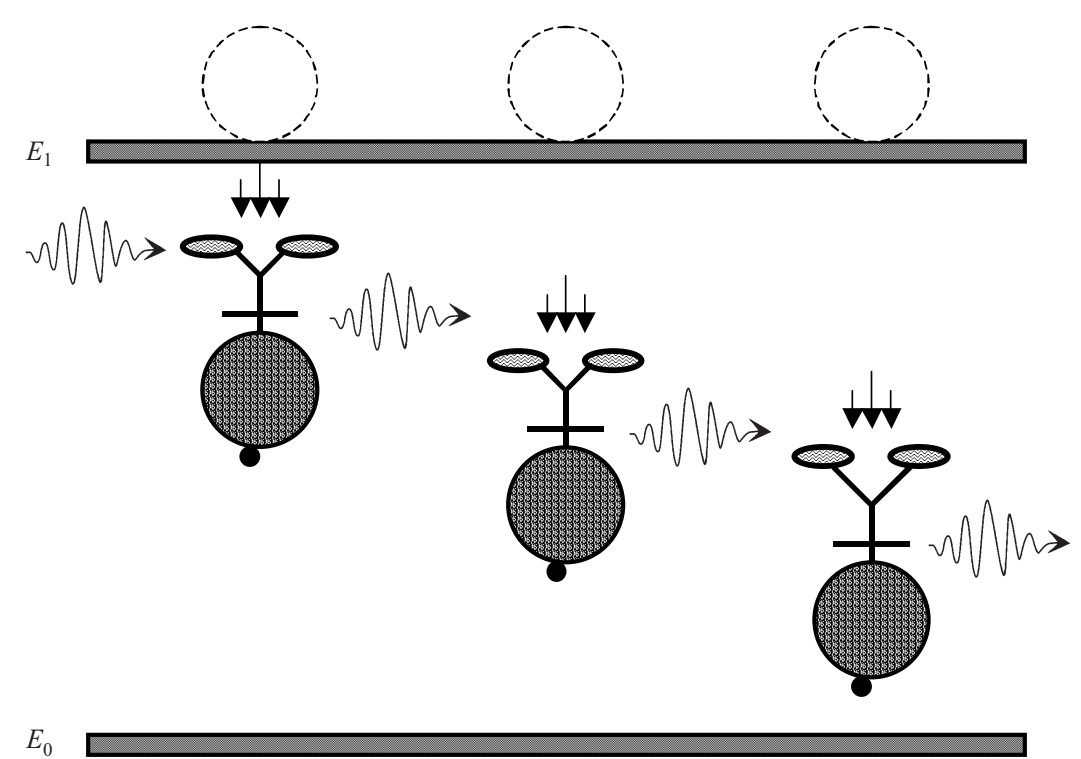

Fig. 2.2 Stimulated emission as coherent monochromatic radiation which consists of the emitted photons that travel in phase

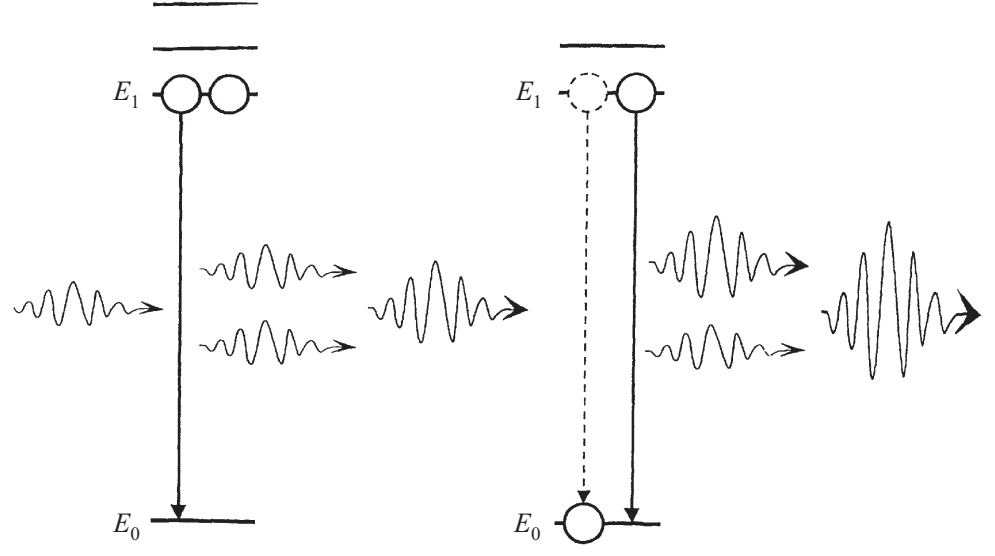

Fig. 2.3 Amplification of the light as a result of travelling of both the incoming and emitted photons in phase, and the appearance of several photons stimulated by a single photon

\subsection{INVERSION OF POPULATION}

The number of photons produced by stimulated emission must exceed the number of photons lost by absorption in order to provide amplification of light. This condition means that the number of excited 


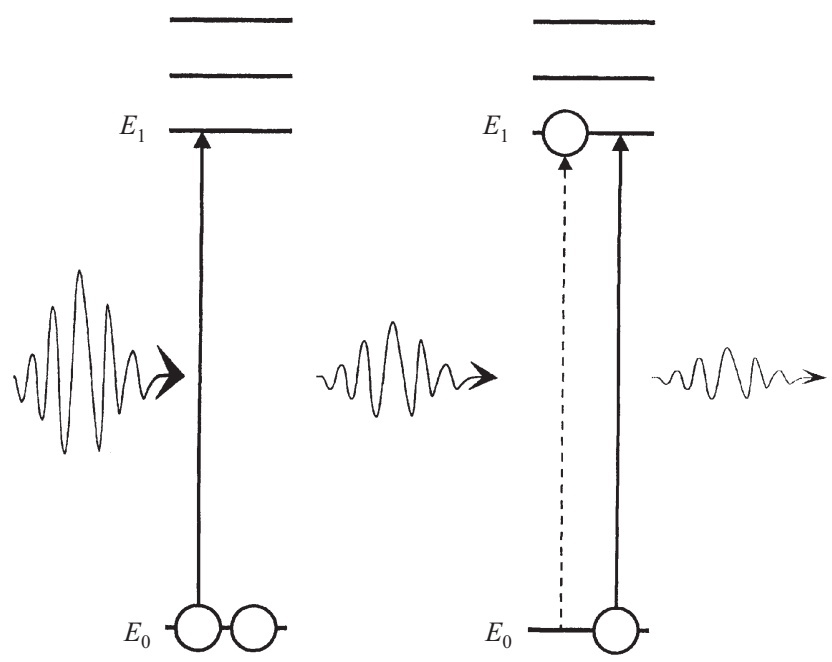

Fig. 2.4 Absorption as the interaction of the external photon with the laser medium; this process induces a transfer of a certain number of particles from $E_{0}$ to $E_{1}$ and is characterized by decreasing of the number of photons

particles must prevail the number of particles in the ground state. Such a situation can be realized due to pumping which causes inversion of population - the redistribution of the particles population between the energy levels (Fig. 2.5).

\subsection{COMPONENTS OF LASER}

The main components of laser are active medium (gas or mixture of gases, solution of organic dye, solid crystal, or semiconductor), optical resonator (two flat or concave mirrors, one of which is semitransparent), and source of pumping (electrical discharge, electron beam, light flash, gas dynamic system, or chemical reactor). Typical design of laser is given in Fig. 2.6.

\subsection{FORMATION OF LASER RADIATION}

Let us consider the behaviour of spontaneously emitted photons in the active medium of laser (Fig. 2.7) [Posudin, 1985; 1988; 1989]. Photon 1 propagates at some angle to the longitudinal axis of the resonator which is formed with two mirrors are numbered $M_{1}$ and $M_{2}$. This photon leaves the resonator and does not take part in laser generation; what happens to it is not dealt with here as it is of no consequence in this context. A similar behaviour is demonstrated by photon 2 after reflection from the 
16 Practical Spectroscopy in Agriculture and Food Science

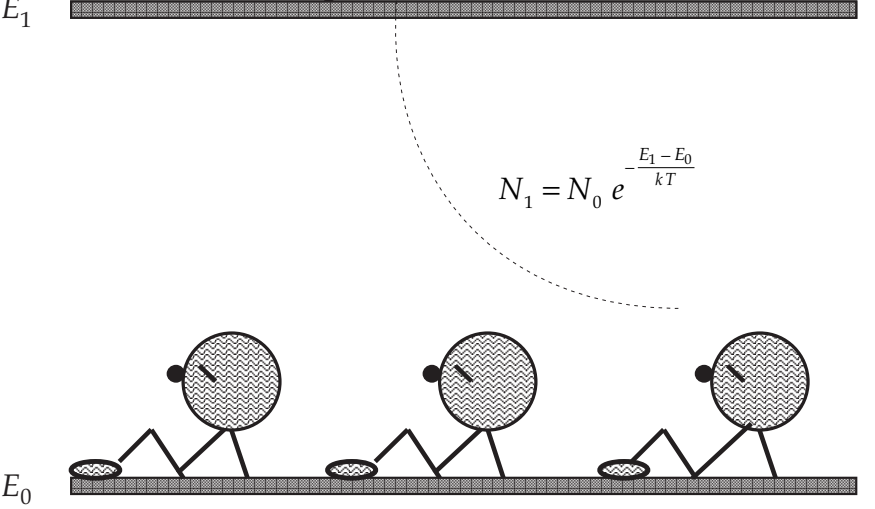

(a)

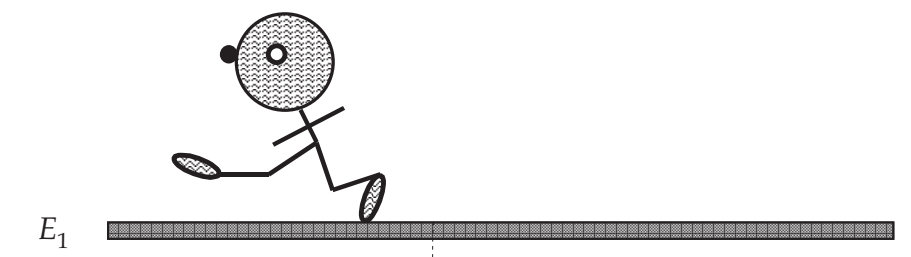

$E_{0}$

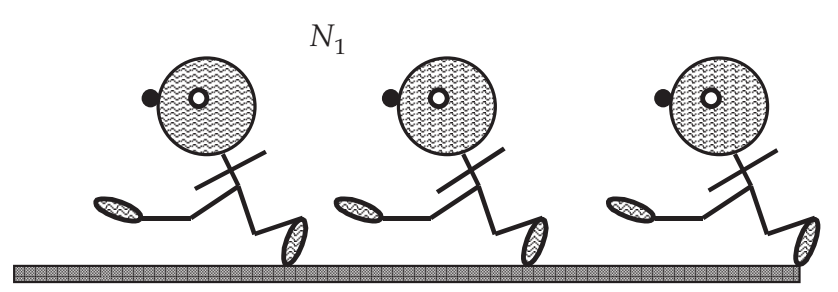

(b)

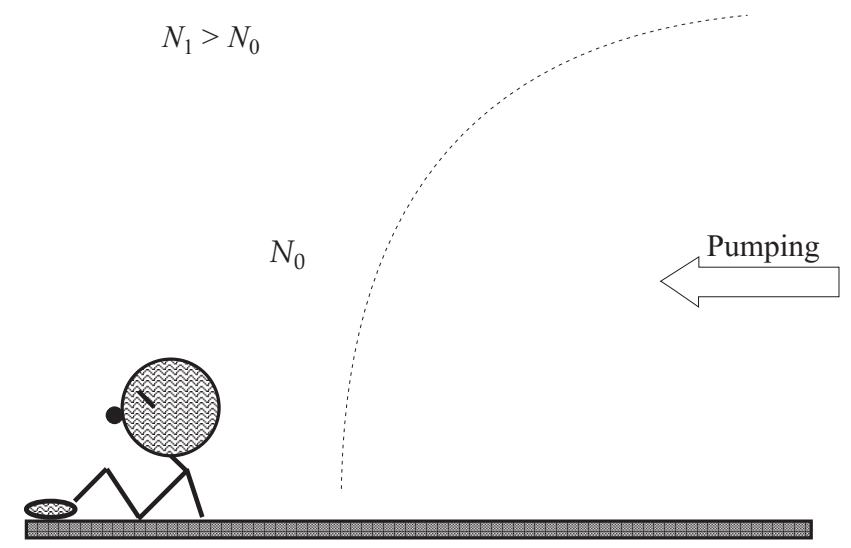

Fig. 2.5 Inversion of population as the redistribution of the particles population between the energy levels $(a)$; this condition means that the number of excited particles, $N_{1}$ must prevail the number of particles in the ground state, $N_{0}$ under the effect of pumping $(b)$ 


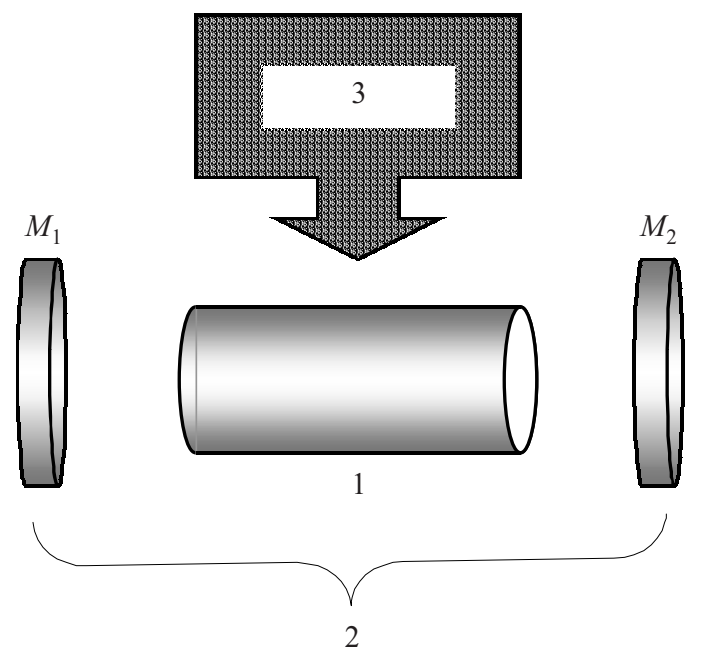

Fig. 2.6 The main components of laser: 1 - active medium (gas or mixture of gases, solution of organic dye, solid crystal, or semiconductor), 2 - optical resonator (two flat or concave mirrors, one of which is semitransparent), and 3 - source of pumping (electrical discharge, electron beam, light flash, gas dynamic system, or chemical reactor)

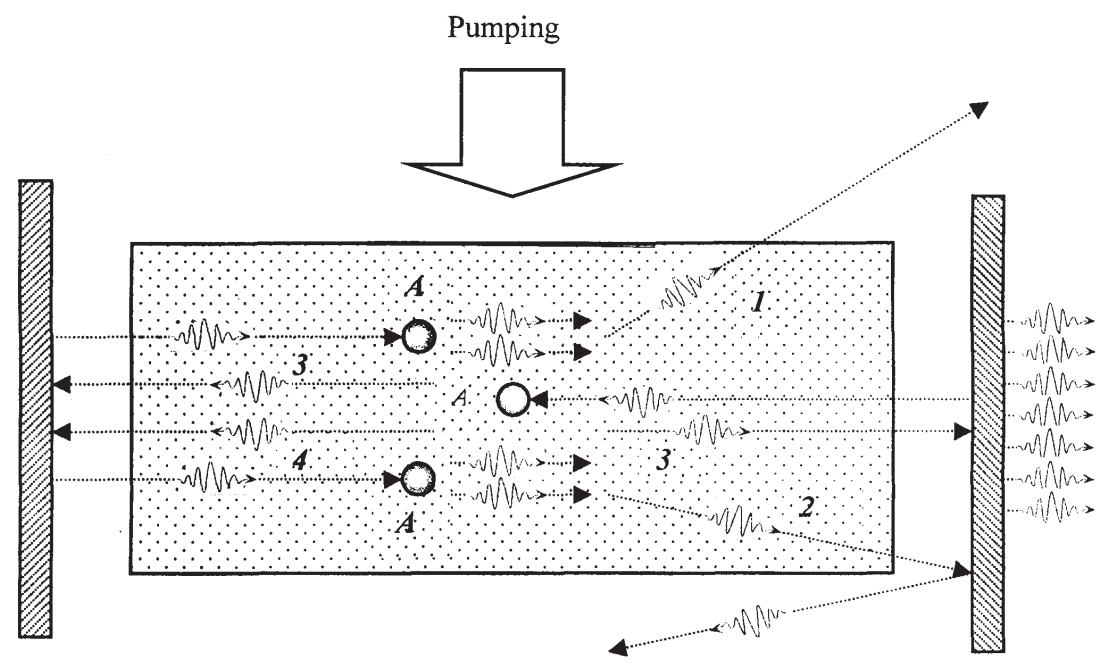

Fig. 2.7 Formation of laser radiation (explanation in text)

surface of the mirror. Photon 3 propagates along the longitudinal axis of the resonator; it reflects from the surface of the mirror, reverses and interacts with the excited particle $A$ (atom or molecule) of the active 


\section{Practical Spectroscopy in Agriculture and Food Science}

medium. This particle is transferred from the excited level to the ground level and emits a new photon 4 as a result of stimulated emission. Both photons 3 and 4 travel in the same direction. They propagate in parallels to the longitudinal axis of the resonator, reflect from mirrors, and the process of stimulated emission is repeated. One of the mirrors has 100\% reflection, and the other is partially transparent. The output laser beam is formed with only those photons whose direction of propagation is in parallels to the longitudinal axis. That is why a laser beam is characterized by a small divergence.

\subsection{PROPERTIES OF LASER RADIATION}

The main properties of laser radiation are monochromaticity, coherence, directionality, and brightness. Some types of lasers have the possibility to change the frequency (or wavelength) of radiation. The other laser devices produce ultrashort pulses of radiation.

Monochromaticity means a narrow spectral interval of radiation, which can be characterized approximately by one frequency (or wavelength) only.

Coherence is the co-ordinated proceeding of several wave processes in space and time. Temporal coherence can be distinguished by the following factor: if for a given point in space there is a constant phase difference between the amplitude of the wave at two successive instances in time; and spatial coherence - which is characterized by a constant timeindependent phase difference for amplitude at two different points.

Directionality is determined by the angular distribution of the laser beam. This property is the direct result of the propagation of two light waves in the optical cavity; only waves, which propagate normally to the resonator mirrors, participate in the formation of a laser beam. The angular distribution, or divergence of gas lasers is about $10^{\prime}$; of solid-state lasers $10^{\prime}-40^{\prime}$; of semiconductor lasers about $30^{\circ}$.

Brightness of the source of electromagnetic waves is defined as the power of radiation which is emitted from the unit of the source surface through the unit solid angle. As soon as the laser beam has a small divergence, it has a brightness which is several orders higher than the traditional sources of light.

\subsection{SUMMARY}

Unique properties of laser radiation such as monochromaticity, coherence, directionality, and brightness provide the performance of 


\section{Practical Spectroscopy in Agriculture and Food Science}

new nondestructive, fast and precise methods for control of agricultural objects.

The progress in the successful realization of laser methods in agriculture is obvious and many convincing examples of this progress are increasing day to day. The sphere of laser technology in agriculture is open to new investigations and practical applications.

\subsection{REFERENCES}

Posudin, Yu.I. 1985. Laser Microfluorometry of Biological Objects. Vyssha Shkola, Kiev. p 108. Posudin, Yu.I. 1988. Lasers in Agriculture. Science Publishers, Inc., Enfield, NH, USA. p 188. Posudin, Yu.I. 1989. Laser Photobiology. Vyssha Shkola, Kiev. p 246.

Svelto, O. 1976. Principles of Lasers. Plenum Press, New York, London. p 373. 


\section{Spectroscopic Methods}

\subsection{ABSORPTION/TRANSMISSION SPECTROSCOPY}

All agricultural objects are built out of smaller particles called molecules and atoms. Each atom in a molecule takes part in vibrational movements with a certain vibrational frequency. If a light wave of a certain frequency interacts with an object that has atoms of the same vibrational frequency, then those atoms will absorb the light energy and transfer their vibrational energy into thermal energy through the interaction with neighbouring atoms. The light wave usually consists of many frequencies; the object absorbs certain frequencies selectively. Different types of atoms and molecules have a different vibrational frequency; that is why they selectively absorb different frequencies of light. For example, chlorophyll absorption of blue and red light means the reflection of green light: hence the green colour of all the leaves can be distinguished.

Let us discuss the main terminology which is related to the passing of light through the object (Fig. 3.1).

Absorption is the process in which incident radiated energy is retained without reflection or transmission on passing through a medium.

Absorbance is the ability of a solution or a layer of a substance to absorb radiation that is expressed mathematically as the negative common logarithm of the transmittance of the substance or solution:

$$
A=-\lg \left(\frac{I_{t}}{I_{0}}\right)=-\lg (1-\alpha)=-\lg \tau=\alpha C l
$$

where $\tau=\frac{I_{t}}{I_{0}}$ is transmittance, $C=$ concentration, $l=$ thickness of the sample (the path length). 
Absorbance is also called optical density $D$, but absorbance is related with absorption inside the substance only, while optical density is determined either by absorption, or by scattering.

Absorptivity (extinction coefficient) is used if concentration $C$ is expressed in $\mathrm{g} / \mathrm{L}$ :

$$
a=\frac{A}{C l}
$$

The unit of absorptivity is $\mathrm{L}^{-1} \cdot \mathrm{cm}^{-1}$.

Molar absorptivity (molar extinction coefficient) is used if concentration is expressed in units of $\mathrm{mol} / \mathrm{L}$ :

$$
\varepsilon=\frac{A}{C l}
$$

The unit of molar absorption coefficient is the $\mathrm{L} \cdot \mathrm{mol}^{-1} \cdot \mathrm{cm}^{-1}$.

It is possible to obtain from the equations (2.1) and (2.3) the important relationship that is known as Beer-Lambert-Bouger Law:

$$
I_{t} / I_{t}=e^{-\varepsilon C l}
$$

Absorption cross section is used sometimes for the description of the absorption properties of a substance:

$$
\sigma=A /(0.434 n l)
$$

where $n$ is the concentration of the substance in atoms or molecules in $\mathrm{cm}^{3}$.

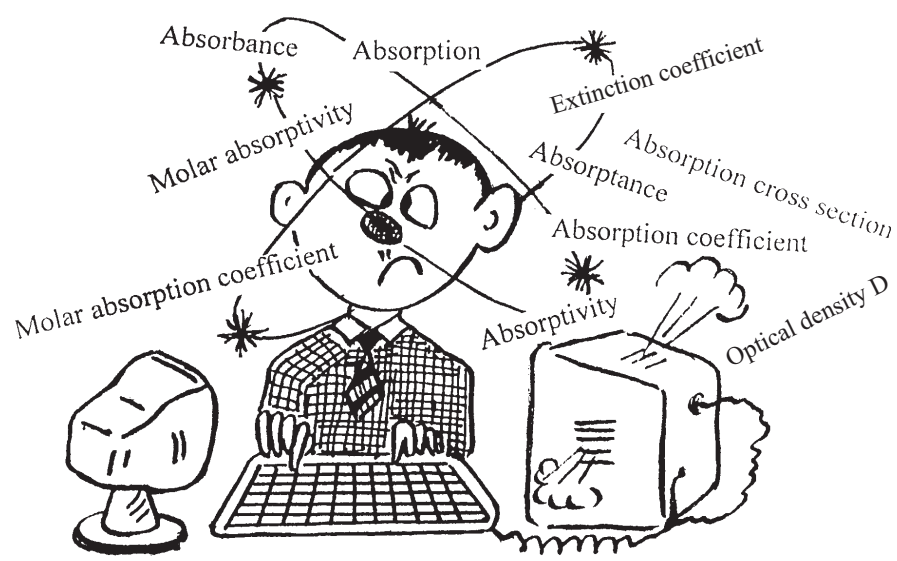

Fig. 3.1 Interaction of light with the objects is characterized with a rich terminology 
Absorption cross section is related to molar absorptivity:

$$
\sigma=3.8 \cdot 10^{-21} \varepsilon
$$

Coefficient of absorption $\alpha$, absorptivity $a$, molar absorptivity $\varepsilon$, and cross section $\sigma$ are related to the absorbance $A$ :

$$
A=0.434 \alpha l=0.434 \sigma n l=a C l=\varepsilon C l
$$

\subsection{LIGHT REFLECTION}

Reflection is the phenomenon of a propagating light wave being thrown back from a surface. Reflection may be specular or diffuse according to the nature of the interface.

Specular reflection is the reflection of light from a surface where at the point of reflection an incident beam is reflected at (and only at) an angle equal to the angle of incidence (both taken with respect to the perpendicular at that point) (Fig. 3.2a).

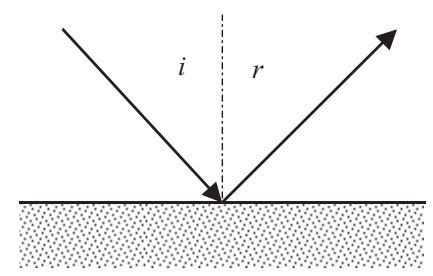

(a)

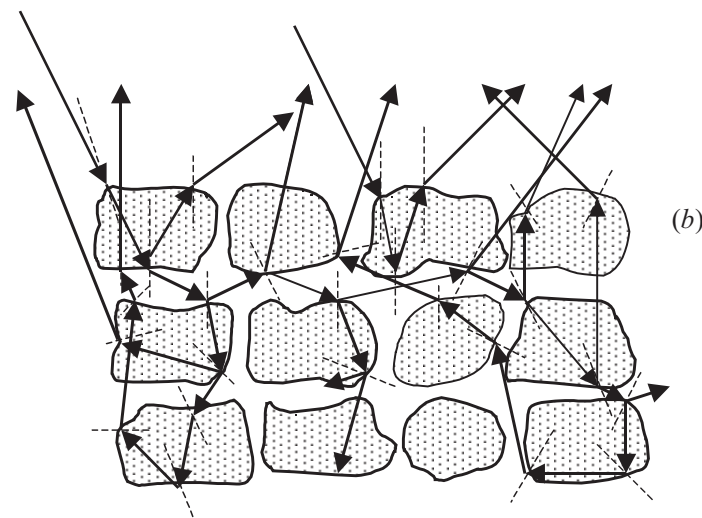

Fig. 3.2 Types of reflection: specular reflection $(a)$ provides little or no information about internal properties of an agricultural object in comparison with diffuse reflection $(b)$ that is received due to numerous processes of random reflection, refraction, and scattering at interfaces of internal elements inside the sample 
Diffuse (or body) reflection is the reflection of light from an uneven or granular surface such that an incident ray is seemingly reflected at a number of angles; whereas specular reflection provides little or no information about internal properties of an agricultural object in comparison with diffuse reflection that is received due to numerous processes of random reflection, refraction, and scattering at interfaces of internal elements inside the sample (Fig. 3.2b).

The following empirical expression can be used for diffuse reflectance $R$ [McClure, 1994]:

$$
D=\ln \frac{1}{R}=\ln \frac{I_{0}}{I_{r}}
$$

The agricultural objects which absorb weakly optical radiation and contain scattering particles are characterized by the effect of intensification of the absorbing bonds: this intensification arises from the radiation traversing an optical path which may be as much as eighty times the sample thickness [Handbook of Near-Infrared Analysis, 1992].

The diffuse reflectance can be estimated in practice through the following equation that demonstrates a nearly linear relationship with concentration [Norris, 1982]:

$$
\lg \frac{R^{\prime}}{R}=\lg \frac{1}{R}+\lg R^{\prime} \sim a C / s
$$

where $R^{\prime}$ and $R=$ reflectance of the standard (barium sulphate or magnesium oxide) and of the sample correspondingly, $a=$ constant, $C=$ concentration, $s=$ scattering constant.

For monochromatic radiation equation (3.9) can be rewritten as:

$$
C=k+(s / a) \lg \left(\frac{1}{R}\right)
$$

where $k$ is absorption constant.

The Near-Infrared (NIR) region of the electromagnetic spectrum occupies the wavelength range from $760 \mathrm{~nm}$ to 2,500 nm (or from 13,000$4,000 \mathrm{~cm}^{-1}$ ). All the absorption bands in the NIR region are induced by many vibrational transitions, particularly higher harmonics of fundamental transitions (overtones) and combinations of these harmonics with fundamental transitions. The shape of the NIR spectrum for a given molecule is sharp, unique and characteristic, and can be used for identification and quantitative analysis of the sample. Typical NIR spectra of milk, cream and sour cream are presented in Figs. 3.3-3.5. 


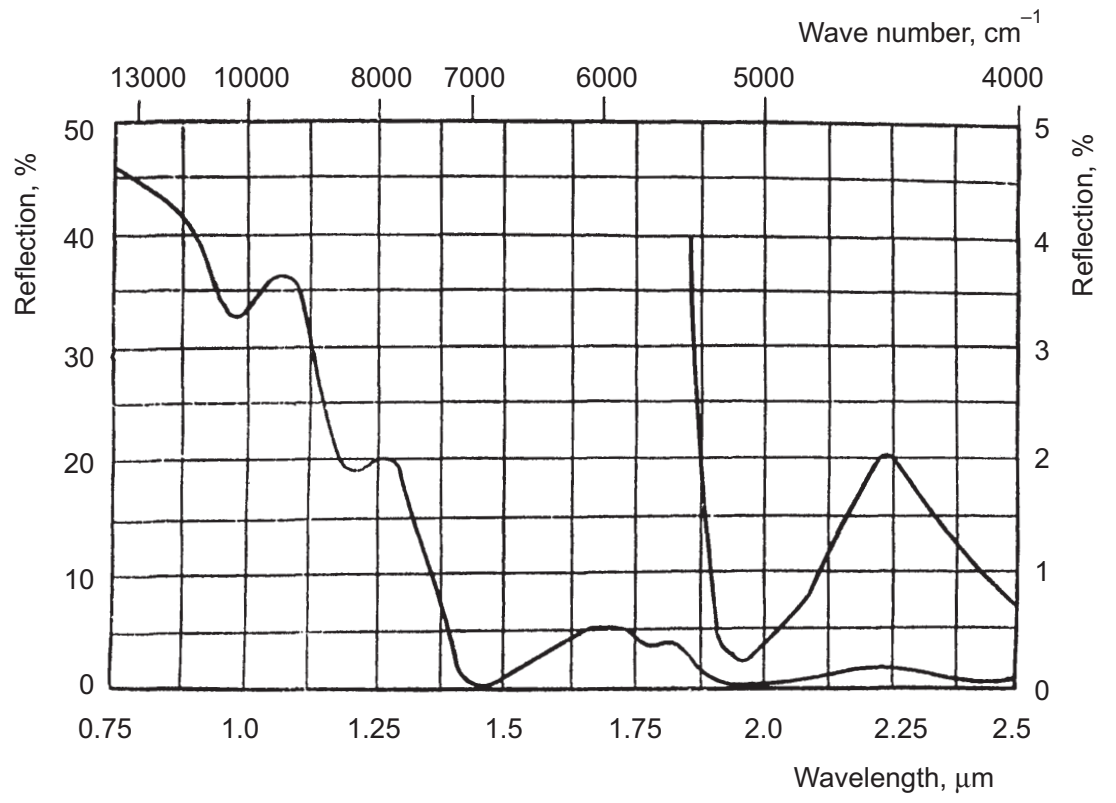

Fig. 3.3 Near-infrared spectra of milk

The presence of characteristic groups such as $-\mathrm{CH},-\mathrm{OH},-\mathrm{NH}$ causes the appearance of overtones: thus, fundamental vibrations of $\mathrm{CH}$-group occupy the wavelength range 3,000-3,600 nm; first overtone $-1,600-1,800$ $\mathrm{nm}$; second overtone $-1,100-1,200 \mathrm{~nm}$. The region of vibrations of $\mathrm{NH}-$ group consists of 2,900 $\mathrm{nm}$ (fundamental vibration), 1,500 nm (first overtone), 1,000 nm (second overtone), and 2,200 nm (combination of overtones). The spectral region of $\mathrm{OH}$-group is presented by $2,800 \mathrm{~nm}$ (fundamental vibration), 1,400 $\mathrm{nm}$ (first overtone), 1,000 nm (second overtone) [Fuhrman, 1987].

In addition, there is participation of water in formation of a NIR spectrum; spectral properties of water are caused by a number of overtones at 760, 970, 1,180, and 12,450 nm, and combination of overtones at $1,940 \mathrm{~nm}$.

\subsection{LUMINESCENCE}

Luminescence occurs when the energy source transfers an electron of an atom from a lower (ground) energy state into an excited higher energy state, then the electron releases the energy in the form of light when it returns back to a lower energy state. This term refers to emission from 


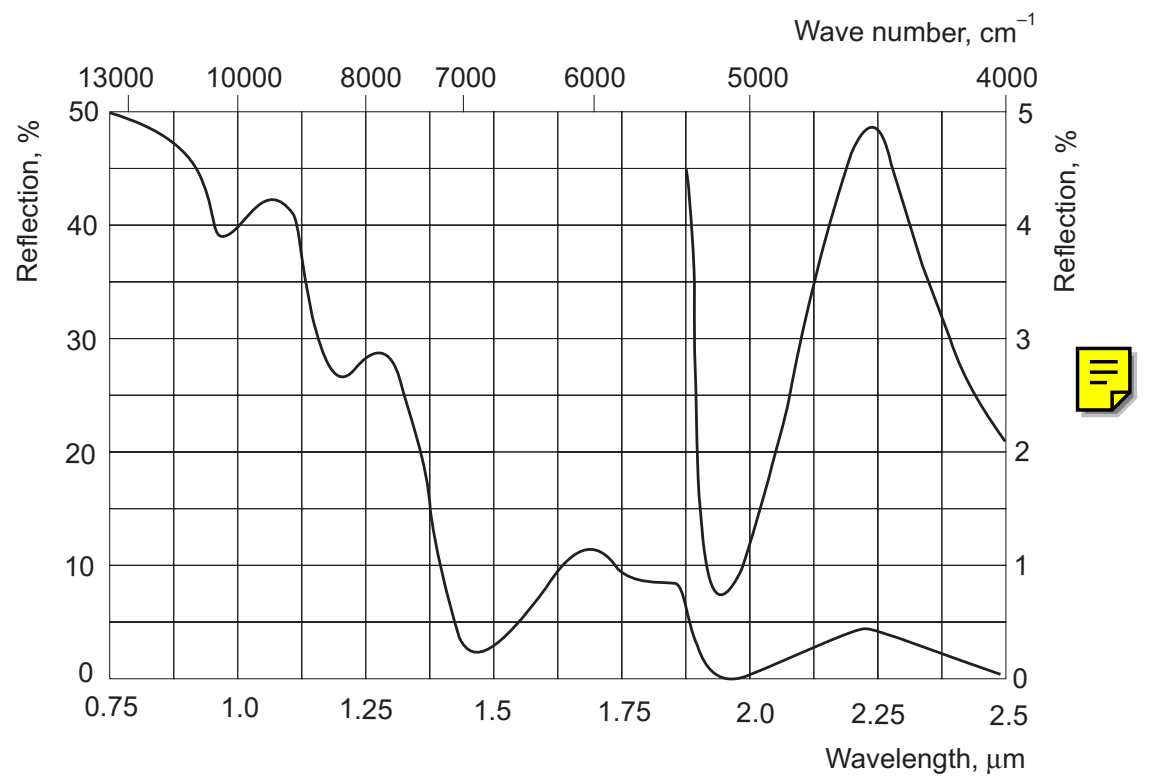

Fig. 3.4 Near-infrared spectra of cream

cool bodies or to emission from hot bodies that is not due to thermal excitation. If the emission of light from excited states is produced by light absorption, such a process is called photoluminescence. There are two particular types of photoluminescence - short-lived $\left(10^{-10}-10^{-6} \mathrm{~s}\right)$ fluorescence and long-lived $\left(10^{-6}-10^{4} \mathrm{~s}\right)$ phosphorescence.

\subsection{SCATTERING}

Light scattering is the change of some characteristics of the light flow (space distribution of light intensity, frequency spectrum, polarization) during its interaction with the matter. Experiments have indicated that the elastic scattering is characterized by the same frequency as that of scattered radiation. This type of light scattering can be classified as Rayleigh scattering, Debye scattering, and Mie scattering. A concrete type of scattering is determined by the refractive indices of the particlescatterers and surrounding medium, and by the dimensions of these particles relative to the wavelength of the incident radiation (Table 3.1).

Each type of light scattering is also characterized by a certain angular distribution of scattered radiation intensity: little particles demonstrate the symmetric distribution of scattered light, while large particles 


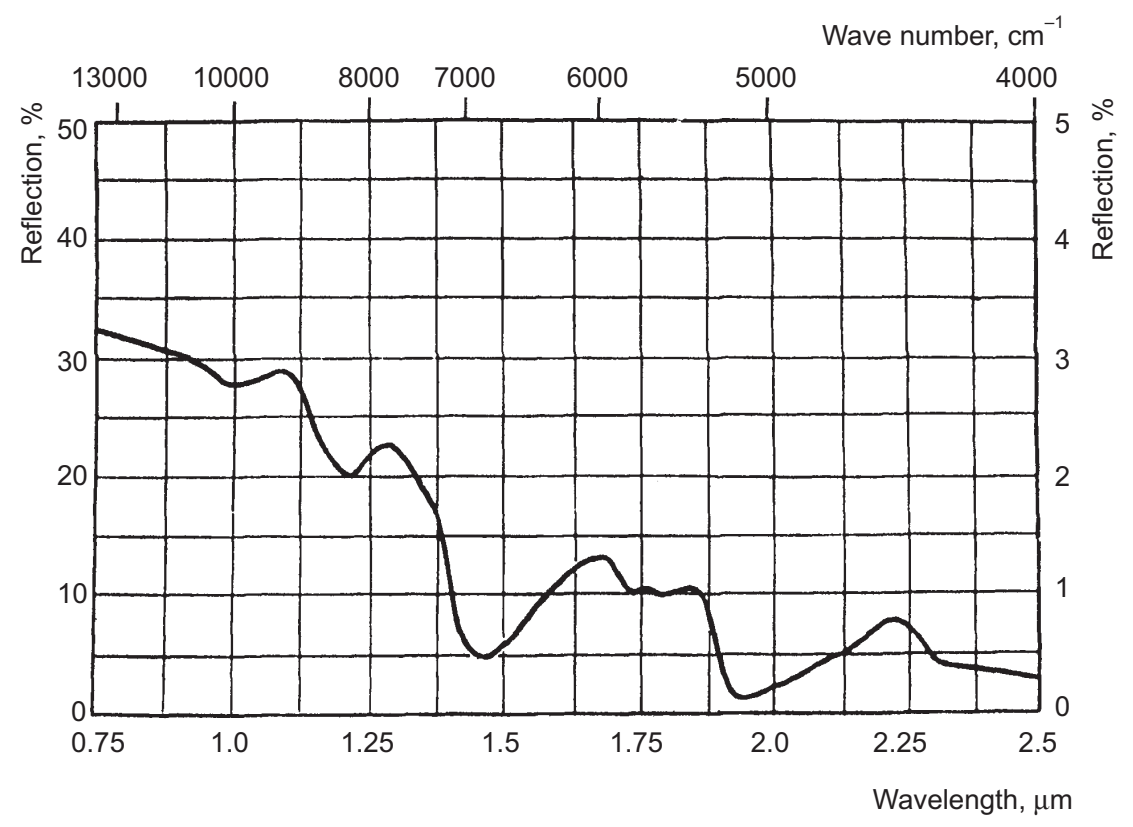

Fig. 3.5 Near-infrared spectra of sour cream

Table 3.1 Types of light scattering [Ingle and Crouch, 1988]

\begin{tabular}{ccc}
\hline Types of Scattering & Refractive Index Requirement & Size Requirement \\
\hline Rayleigh & $|(n-1)|<<1$ & $d<0.05 \lambda$ \\
Debye & $|(n-1)| \approx 0.1$ & $0.05 \lambda<d<\lambda$ \\
Mie & $(n-1)>>0$ & $d>\lambda$ \\
\hline
\end{tabular}

produce the presence of a number of maxima and minima due to interference between the rays reflected from different points of the particle (Fig. 3.6). Large-particle scattering is used in turbidimetry and nephelometry - methods for determining the amount of cloudiness, or turbidity, in a solution based on measurement of the effect of this turbidity on the transmission and scattering light.

\subsection{SUMMARY}

Spectroscopic methods are based on the interaction of optical radiation with matter. Such types of interaction can be distinguished as absorption 

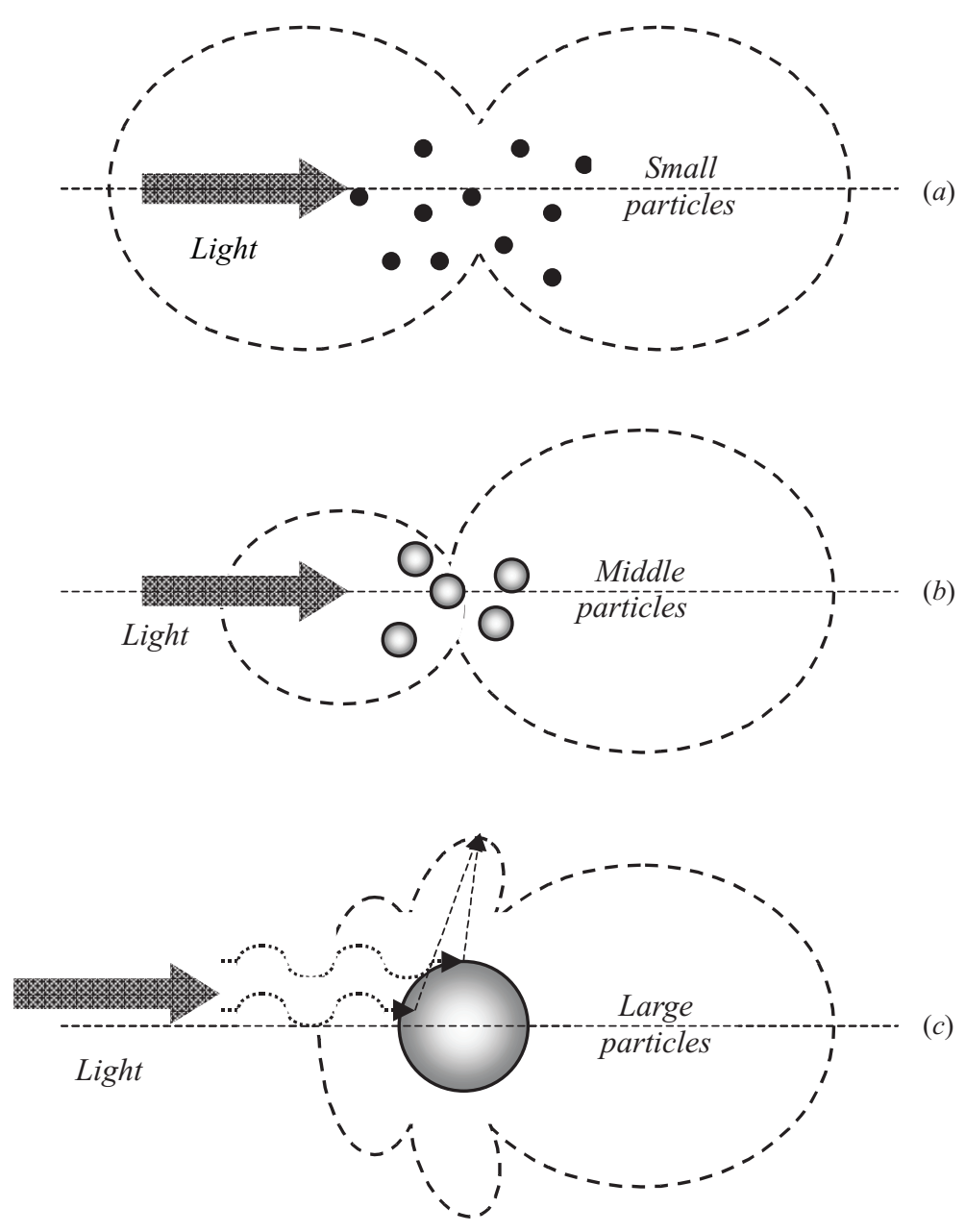

Fig. 3.6 Each type of light scattering is characterized by a certain angular distribution of scattered radiation intensity: $a$ - little particles demonstrate the symmetric distribution (Rayleigh scattering); $b$ - middle particles produce asymmetric distribution (Debye scattering); $c$ - large particles produce the presence of a number of maxima and minima due to interference between the rays reflected from different points of the particle (Mie scattering)

- the process in which incident radiated energy is retained without reflection or transmission on passing through a medium; reflection - the phenomenon of a propagating light wave being thrown back from a surface; luminescence which occurs when the energy source transfers an electron of an atom from a lower (ground) energy state into an excited 


\section{Practical Spectroscopy in Agriculture and Food Science}

higher energy state, then the electron releases the energy in the form of light when it returns back to a lower energy state; light scattering - the change of some characteristics of the light flow (space distribution of light intensity, frequency spectrum, polarization) during its interaction with matter. All spectroscopic methods offer non-destructive, rapid and precise evaluation of agricultural products.

\subsection{REFERENCES}

Burns, D.A. and Ciurczak, E.W., eds. 1992. Handbook of Near-Infrared Analysis. Marcel Dekker, Inc., New York-Basel-Hong Kong. p 681.

Fuhrman, I. 1987. Zur anwendung der nah-infrarotreflexions-spektroscopie in der milchwirtschaft. Lebensmit teilindustrie.4:171-174.

Galen Wood Ewing, ed. 1997. Analytical Instrumentation Handbook. Marcel Dekker, Inc., New York-Basel. p 1453.

Ingle, J.D. and Crouch, S.R. 1988. Spectrochemical Analysis. Prentice Hall, Englewood Cliffs, New Jersey. p 590.

McClure, W.F. 1994. Near-Infrared Spectroscopy. In: Spectroscopic Techniques for Food Analysis, R.H. Wilson, ed. VCH Publishers, Inc., New York. pp. 13-57.

Norris, K.H. 1982. Multivariate Analysis of New Materials. In: Chemistry and World Food Supplies: The New Frontiers. L.W. Shemit, ed. CHEMRAWN II. Pergamon Press, Oxford, England. p. 527.

Posudin, Yu.I. 1996. Methods of Optical and Laser Spectroscopy in Agriculture. Seminar Series of Fulbright Scholar. University of Georgia, Athens, U.S.A. 


\section{Spectroscopic Analysis of Milk and Dairy Products}

\subsection{COMPOSITION OF MILK}

Milk is a complex mixture of fat, protein, lactose, vitamins, salts and other compounds which can exist in colloidal dispersion or in an aqueous solution of milk components. Approximate composition of milk of European breeds includes $87.00 \%$ water, $3.80 \%$ non-fat solids, $3.40 \%$ protein, $4.50 \%$ lactose and $1.30 \%$ solids.

Fat of milk is a mixture of glycerides and fatty acids. Fatty acids include polynonsaturated linoleic (two double bonds), linolenic (three double bonds) and arachidonic (four double bonds) acids. Fat globules in milk range from 1 to $10 \mathrm{~mm}$ with a density of (1.5-3.0) $10^{9} \mathrm{ml}^{-1}$.

Proteins consist of about 20 amino acids - the most important being tryptophan, tyrosine and phenylalanine. Proteins in milk are presented by casein micelles $(0.1-0.2 \mu \mathrm{m})$ and serum protein particles $(0.01-0.02$ $\mu \mathrm{m})$.

Carbohydrates in milk are present in the form of lactose (disaccharide), glucose and galactose (monosaccharides).

Milk contains vitamins such as $A$ (retinol), $D$ (ergocalcipherol $D_{2}$ and cholecalcipherol $D_{3}$ ), $K, B_{1}$ (thiomin), $B_{2}$ (riboflavin), niacin (nicotinic acid), pantothenic acid, $B_{6}$, biotin, folic acid, $B$ olabalamin), and $C$ (ascorbic acid).

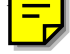

Total solids content is the mass remaining after completion of the specified drying procedure and expressed as a percentage of mass. 
The total non-fat solids means the content of total solids minus the content of fat.

\subsection{INFRARED SPECTROPHOTOMETRY OF MILK AND DAIRY PRODUCTS}

Spectrophotometry is a study of absorption (transmission) properties of a sample as a function of wavelength.

Infrared absorption of milk is related to the presence of certain structural groups in it [Goulden, 1964; Biggs and Sjaunja, 1987]. Each fat molecule consists of three carbonyl groups $(\mathrm{C}=\mathrm{O})$ of triglyceride which are responsible for absorption at $5.73 \mu \mathrm{m}\left(1,745 \mathrm{~cm}^{-1}\right)$; in addition, carbon-hydrogen groups $\left(\mathrm{CH}_{2}\right)$ of triglyceride take part in absorption at 3.4-3.5 $\mu \mathrm{m}\left(2,941-2,857 \mathrm{~cm}^{-1}\right)$. Each molecule of protein consists of amide units which are linked by peptide bonds; the absorption of the amide II band at $6.46 \mu \mathrm{m}\left(1,548 \mathrm{~cm}^{-1}\right)$ can be used for determination of the total protein content. Each molecule of lactose consists of hydroxyl groups $(\mathrm{OH})$ which absorb at $9.6 \mu \mathrm{m}\left(1,042 \mathrm{~cm}^{-1}\right)$. Besides, a molecule of water absorbs at $4.3 \mu \mathrm{m}\left(2,326 \mathrm{~cm}^{-1}\right)$.

\subsection{INSTRUMENTATION}

\subsubsection{Optical Density of Milk}

The measurement of milk particles concentration demands the elimination of light scattering - the condition of unitary scattering of optical radiation by milk particles can be performed if:

(1) the value of optical density is equal $D=\lg I / I_{0}<<1$;

(2) there is a linear dependence of optical density on the concentration of milk particles $D=f(C)$, if $D<<1$.

The dependence of $I / I_{0}$ and $\lg I / I_{0}$ on the level of dilution of milk sample was measured with the photocalorimeter KFK-2MP in spectral region 315-980 nm. The results are shown in Figs. 4.1 $a$ and $b$. It is clear that the dependence $D=f(C)$ demonstrates a linear character for diluted samples of milk.

The relation of optical density of milk and wavelength of optical radiation is presented in Fig. 4.2. The spectral curve has a maximum near 590-600 nm which is probably the result of competition of two processes - scattering by milk particles and natural absorption of milk. 

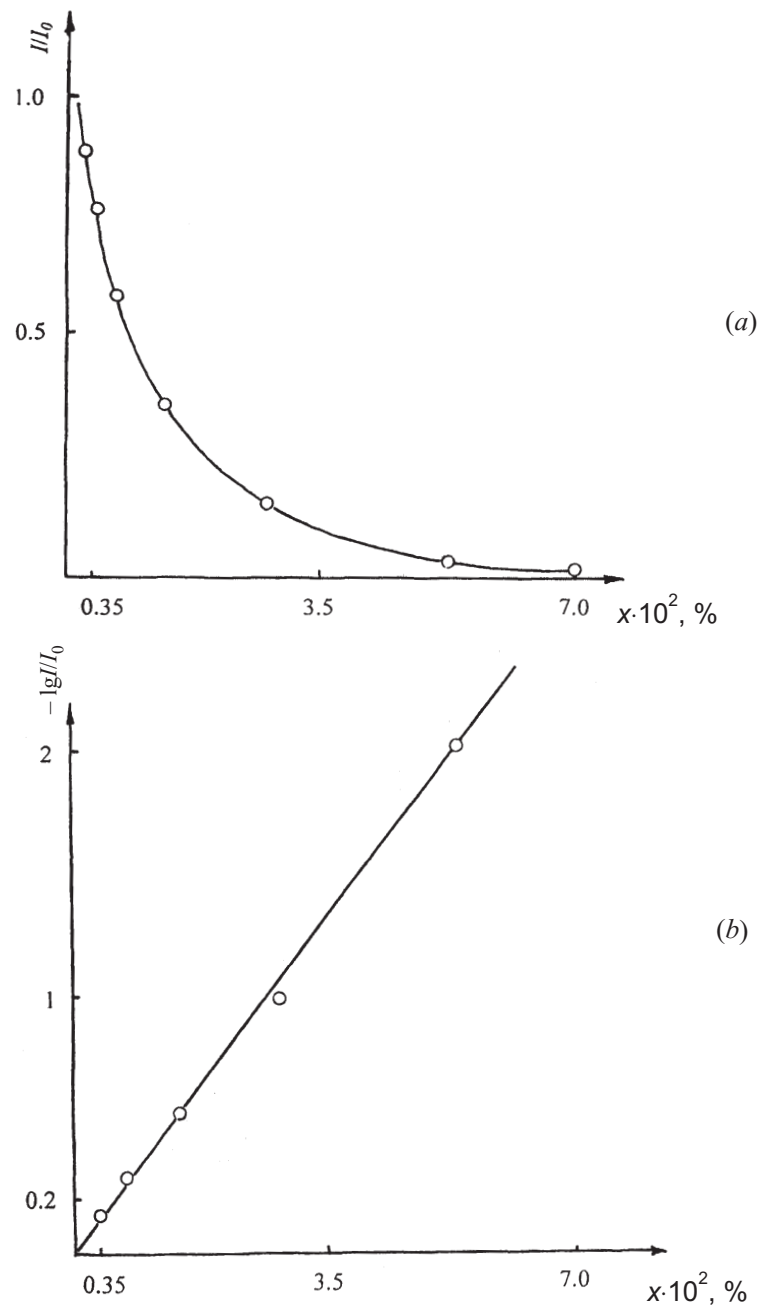

Fig. 4.1 Dependence of $I / I_{0}(a)$ and $\lg / / I_{O}(b)$ on the level of dilution of milk sample

The main conclusion of this investigation is as follows: a milk sample should be diluted adequately to obtain the linear dependence of optical density on the concentration of milk sample which is to be evaluated. This is a serious limitation to the method of absorption/transmission of a spectroscopy in the visible part of the spectrum.

\subsubsection{Infrared Spectrophotometry of Milk and Dairy Products}

The main objective of this investigation is to study the characteristic infrared spectra of milk, boiled butter, casein, and lactose. An infrared 


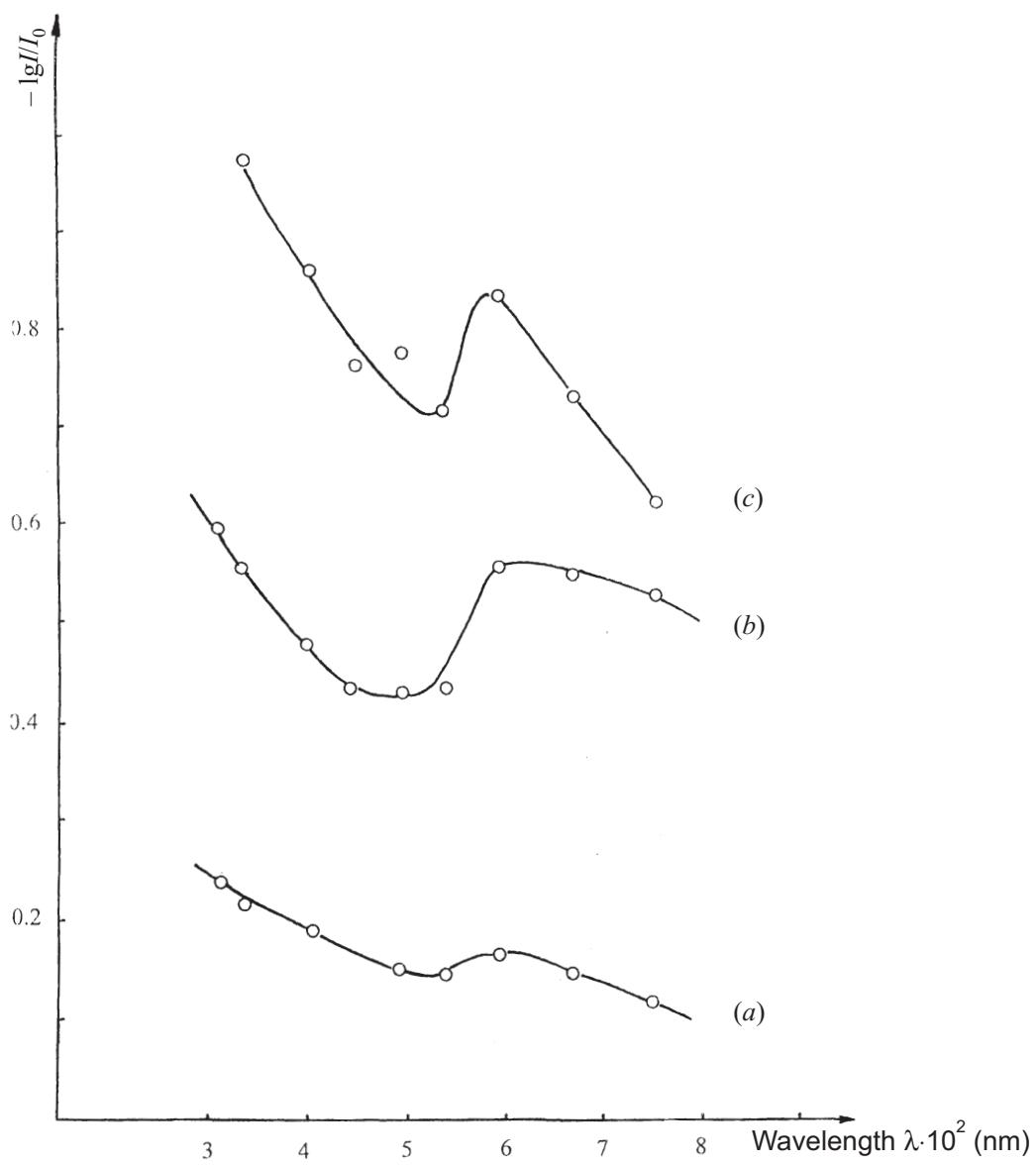

Fig. 4.2 The relation of optical density of milk and wavelength of optical radiation

spectrophotometer UR-20 was used for measurements and it was equipped with a $\mathrm{CaF}_{2}$ cuvette. The collimated infrared radiation passed through the sample, the thickness of which was about several micrometers. The spectral range was $800-5,000 \mathrm{~cm}^{-1}(12.5-2 \mu \mathrm{m})$. The spectrum was recorded with the milk sample in place, and then with a reference sample with water substituting the sample, in order to eliminate the effect caused by the presence of water in milk samples, on the results of measurements. 


\subsection{RESULTS OF INFRARED SPECTROPHOTOMETRY OF MILK AND DAIRY PRODUCTS}

Typical infrared (IR) absorption spectra of milk (3.9\% fat), cream (10\% fat) and sour cream (20\% fat) are presented in Figs. 4.3-4.5; results of measurements of absorption bands are recorded in Table 4.1.

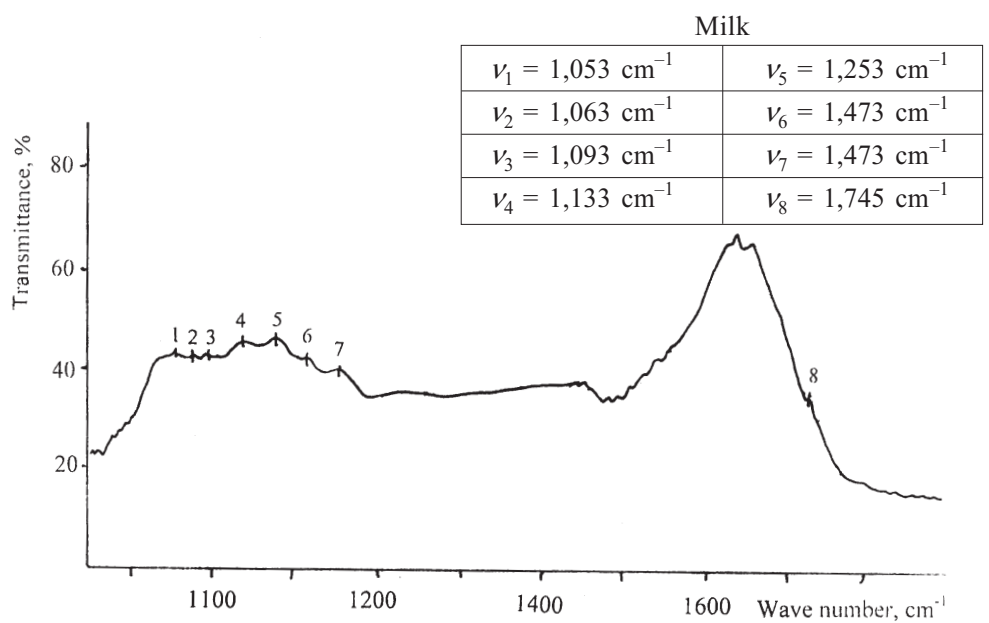

Fig. 4.3 Infrared absorption spectrum of milk (3.9\% fat)

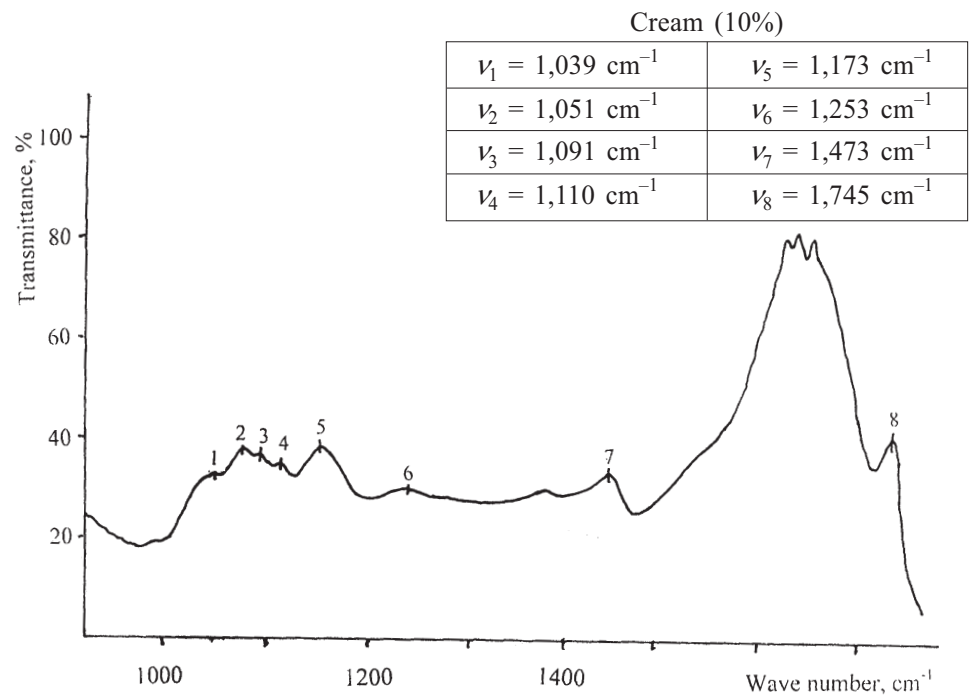

Fig. 4.4 Infrared absorption spectrum of cream (10\% fat) 


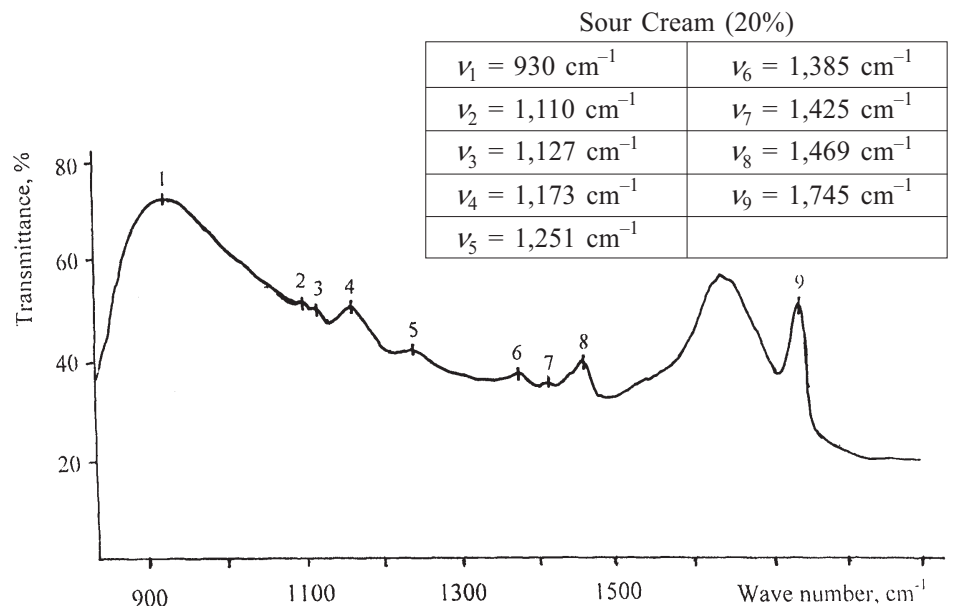

Fig. 4.5 Infrared absorption spectrum of sour cream ( $20 \%$ fat)

Table 4.1 Absorption bands of milk and dairy products [Posudin and Kostenko, 1994]

\begin{tabular}{c|c|c|c|c|c|c}
\hline \multirow{2}{*}{$\begin{array}{c}\text { Absorption } \\
\text { Band }\end{array}$} & \begin{tabular}{c} 
Milk \\
\cline { 2 - 7 } \\
$\begin{array}{c}\text { Number } \\
\left(\mathrm{cm}^{-1}\right)\end{array}$
\end{tabular} & $\begin{array}{c}\text { Wavelength } \\
(\mu \mathrm{m})\end{array}$ & $\begin{array}{c}\text { Wave } \\
\text { Number } \\
\left(\mathrm{cm}^{-1}\right)\end{array}$ & $\begin{array}{c}\text { Wavelength } \\
(\mu \mathrm{m})\end{array}$ & $\begin{array}{c}\text { Wave } \\
\text { Number } \\
\left(\mathrm{cm}^{-1}\right)\end{array}$ & $\begin{array}{c}\text { Wavelength } \\
(\mu \mathrm{m})\end{array}$ \\
\hline$v_{1}$ & 1,053 & 9.50 & 1,039 & 9.62 & 930 & 10.75 \\
$v_{2}$ & 1,063 & 9.41 & 1,051 & 9.51 & 1,110 & 9.01 \\
$v_{3}$ & 1,093 & 9.15 & 1,091 & 9.17 & 1,127 & 8.87 \\
$v_{4}$ & 1,133 & 8.83 & 1,110 & 9.01 & 1,173 & 8.53 \\
$v_{5}$ & 1,173 & 8.53 & 1,173 & 8.53 & 1,251 & 7.99 \\
$v_{6}$ & 1,253 & 7.98 & 1,253 & 7.98 & 1,385 & 7.22 \\
$v_{7}$ & 1,473 & 6.79 & 1,473 & 6.79 & 1,425 & 7.02 \\
$v_{8}$ & 1,745 & 5.73 & 1,745 & 5.73 & 1,469 & 6.81 \\
$v_{9}$ & - & - & - & - & 1,745 & 5.73 \\
\hline
\end{tabular}

Infrared spectra of boiled butter (Fig. 4.6) and aqueous solutions of lactose (Fig. 4.7) were also investigated; the values of absorption bands are given in Table 4.2. The position of absorption bands of aqueous solutions of lactose is stable in the range of solution concentration 0.1-0.8 $\mathrm{mol} / \mathrm{L}$.

It was shown that the position of absorption maxima $v_{i}$ of milk did not demonstrate any major spectral shifts at a given temperature, $\mathrm{pH}$ and chemical composition of the samples within the limits of measurement errors. The concentration $C$ of fat is the most important factor that 


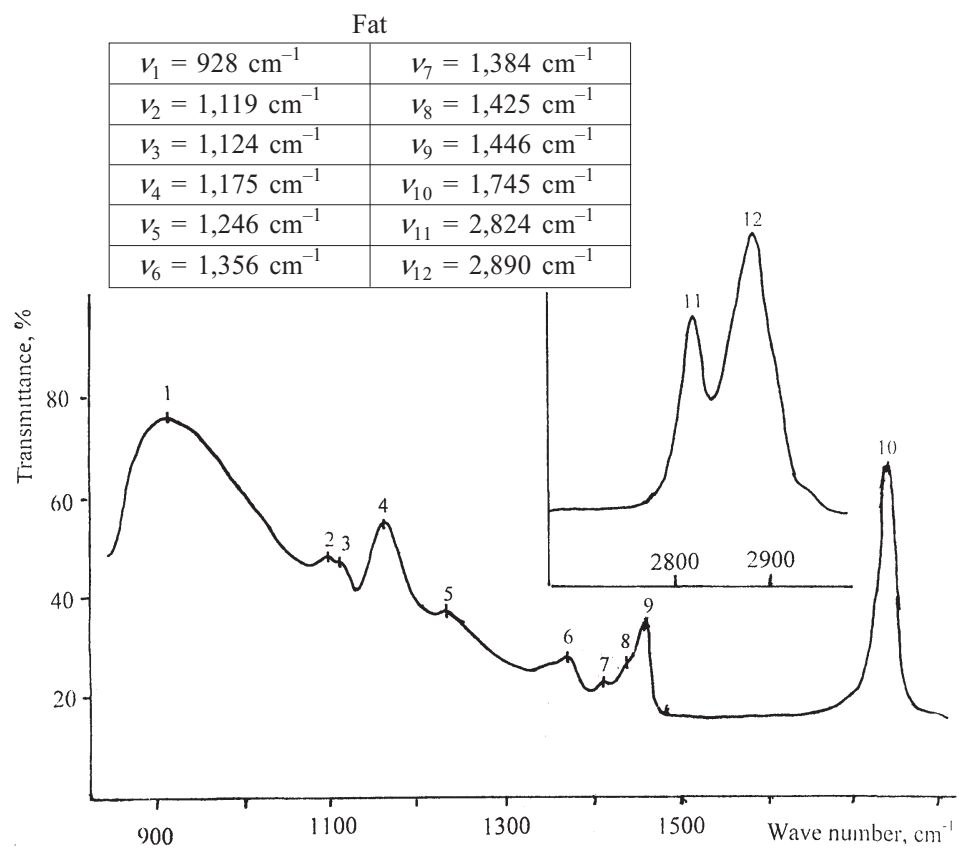

Fig. 4.6 Infrared spectrum of boiled butter

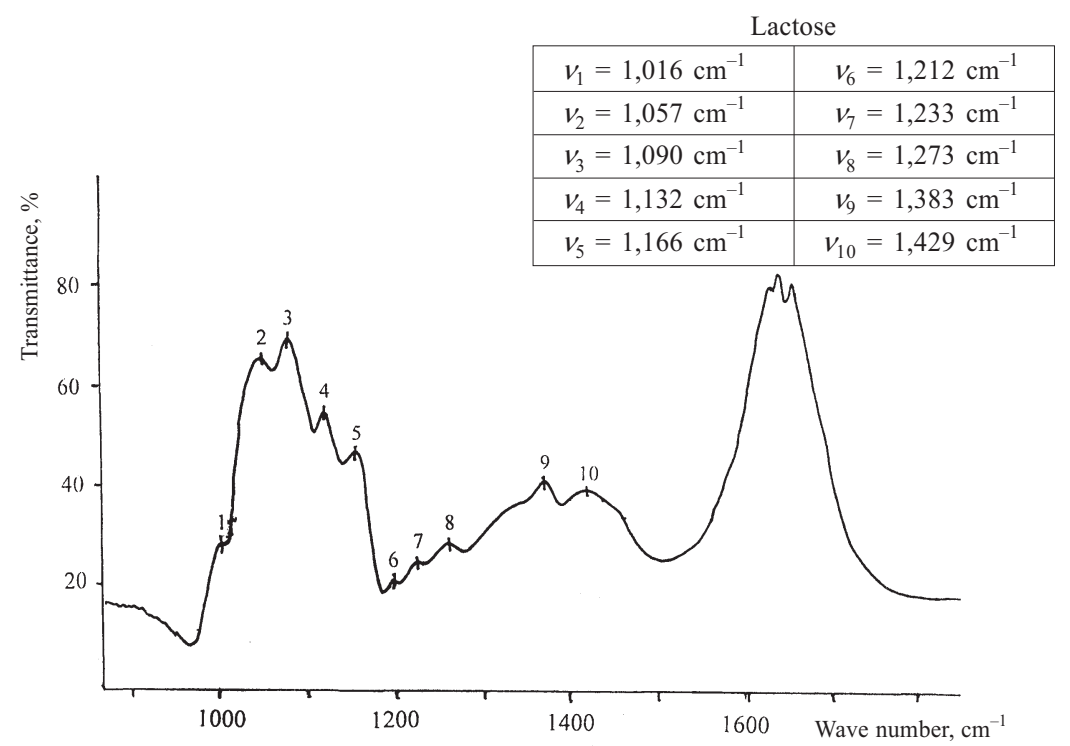

Fig. 4.7 Infrared spectrum of aqueous solutions of lactose 
Table 4.2 Absorption bands of boiled butter and aqueous solutions of lactose [Posudin and Kostenko, 1994]

\begin{tabular}{c|c|c|c|c}
\hline \multirow{2}{*}{ Absorption Band } & \multicolumn{2}{|c|}{ Boiled Butter } & \multicolumn{2}{c}{ Lactose Solution } \\
\cline { 2 - 5 } & $\begin{array}{c}\text { Wave Number } \\
\left(\mathrm{cm}^{-1}\right)\end{array}$ & $\begin{array}{c}\text { Wavelength } \\
(\mu \mathrm{m})\end{array}$ & $\begin{array}{c}\text { Wave Number } \\
\left(\mathrm{cm}^{-1}\right)\end{array}$ & $\begin{array}{c}\text { Wavelength } \\
(\mu \mathrm{m})\end{array}$ \\
\hline$v_{1}$ & 928 & 10.78 & 1,016 & 9.84 \\
$v_{2}$ & 1,119 & 8.94 & 1,057 & 9.46 \\
$v_{3}$ & 1,124 & 8.90 & 1,090 & 9.17 \\
$v_{4}$ & 1,175 & 8.51 & 1,132 & 8.83 \\
$v_{5}$ & 1,246 & 8.03 & 1,166 & 8.58 \\
$v_{6}$ & 1,356 & 7.37 & 1,212 & 8.25 \\
$v_{7}$ & 1,384 & 7.23 & 1,233 & 8.11 \\
$v_{8}$ & 1,425 & 7.02 & 1,273 & 7.86 \\
$v_{9}$ & 1,446 & 6.92 & 1,383 & 7.23 \\
$v_{10}$ & 1,745 & 5.73 & - & - \\
$v_{11}$ & 2,824 & 3.54 & - & - \\
$v_{12}$ & 2,890 & 3.46 & - & - \\
\hline
\end{tabular}

provides effect on position of absorption bands (Fig. 4.8) and half-width of spectral line (Fig. 4.9) (the most isolated line $v_{8}=1,745 \mathrm{~cm}^{-1}$ of milk has been chosen). The decreasing of fat concentration provokes the broadening of initial half-width $\left(21.1 \mathrm{~cm}^{-1}\right)$ and its shift to shortwavelength region. It is interesting to note that the dependence of the ratio $D(1,473) / D(1,745)$ on the fat concentration demonstrates a linear character (Fig. 4.10); it is possible to use this ratio as a spectral index during measurement of fat in milk samples or for calibration procedure.

The process of turning sour causes the deformation of infrared absorption spectra and appearance of a broad maximum near $960 \mathrm{~cm}^{-1}$ (Fig. 4.11).

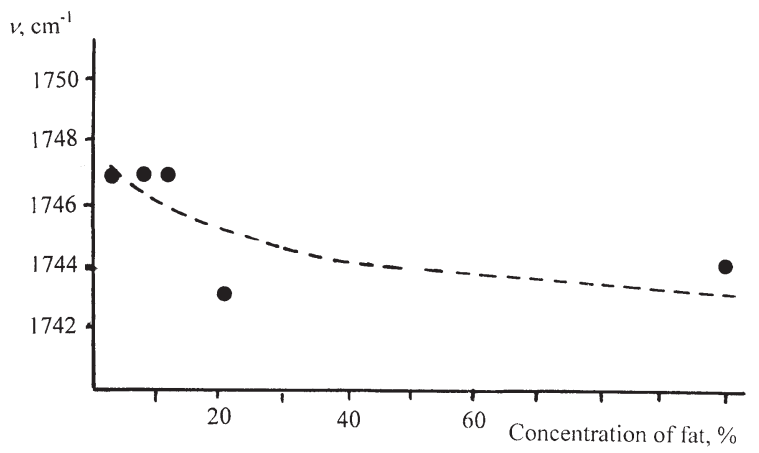

Fig. 4.8 Dependence of the position of absorption bands on the concentration of fat $C$ (spectral line $v_{8}=1745 \mathrm{~cm}^{-1}$ of milk) 


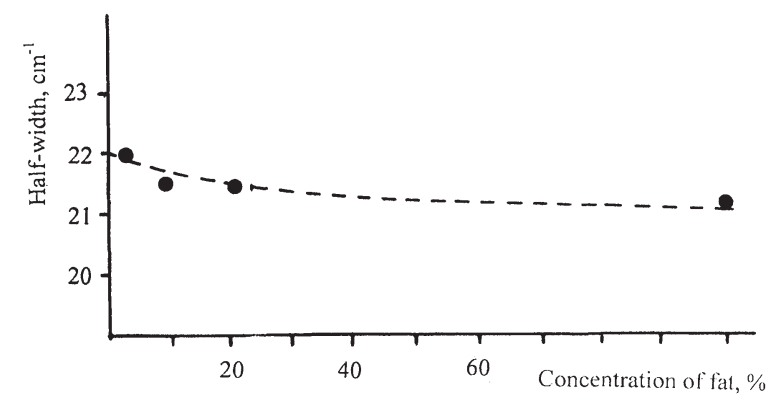

Fig. 4.9 Dependence of the half-width of spectral line $\left(v_{8}=1745 \mathrm{~cm}^{-1}\right)$ of milk on the concentration of fat $C$

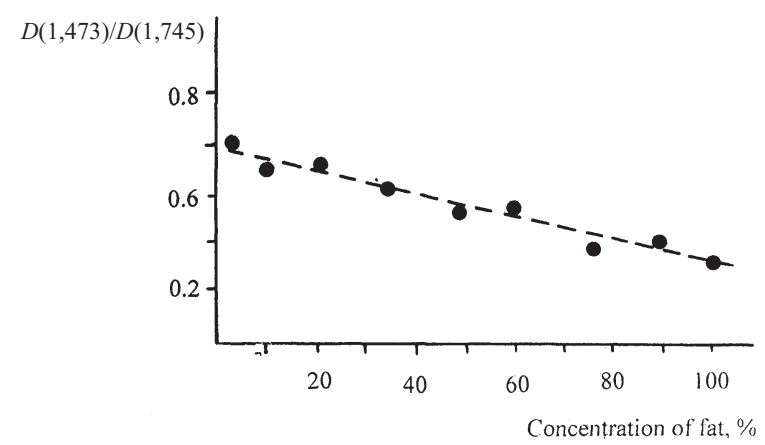

Fig. 4.10 Linear character of dependence of ratio $D(1,473) / D(1,745)$ on the fat concentration

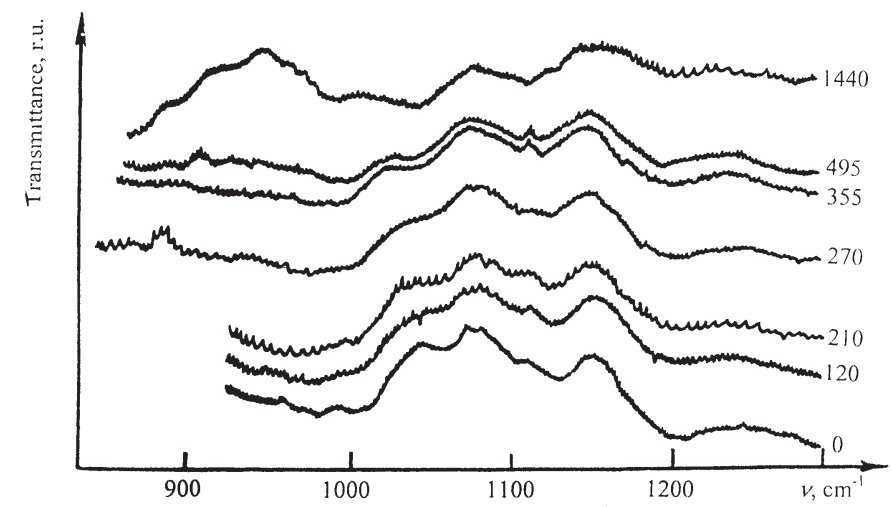

Fig. 4.11 The deformation of infrared absorption spectra and appearance of a broad maximum near $960 \mathrm{~cm}^{-1}$ due to the process of turning sour 


\subsection{INFRARED SPECTROPHOTOMETER FOR MILK ANALYSIS}

An optical design of infrared spectrophotometer for milk analysis [Posudin and Kostenko, 1989, 1992, 1994; Martynenko et al., 1996; Posudin and Timoshenko, 1991; Posudin et al., 1997; Posudin, 2002, 2005] is illustrated in Fig. 4.12. The instrument consists of source 1 of infrared radiation, concave mirror 2, diaphragm 3, modulator 4 of radiation, lens 5 , cuvette 6 with a sample, interference filter 7 , detector 8 , power supply 9 and readout system 10 .

Source of Infrared Radiation. A nichrome coiled filament that was used as the main component of the source of infrared radiation is characterized by the following parameters: voltage 6-8 V; electric current 2.5-3.0 A; power 15-24 W. Maximum of spectral emission is near 3-6 $\mu \mathrm{m}$. Diameter of filament $0.4 \mathrm{~mm}$, and the length $23 \mathrm{~mm}$; diameter and length of the coil 5 and $12 \mathrm{~mm}$ correspondingly. The source was located in the focus of a concave aluminium mirror to provide maximal reflection and radiation density.

A Sample Cuvette. The windows of cuvette (thickness $300 \mu \mathrm{m}$ ) were performed with germanium; the total transmission of such a system was $70 \%$. The air gap between the windows was equal $150 \mu \mathrm{m}$; such a value made it possible to avoid possible interference effects with a narrow gap and to keep the sensitivity of readout system.

Interference Filters. It was necessary to use the interference filters which were tuned at the maximum and at the base of absorption band of each component of milk (Fig. 4.13). The main parameters of interference filters are presented in Table 4.3.

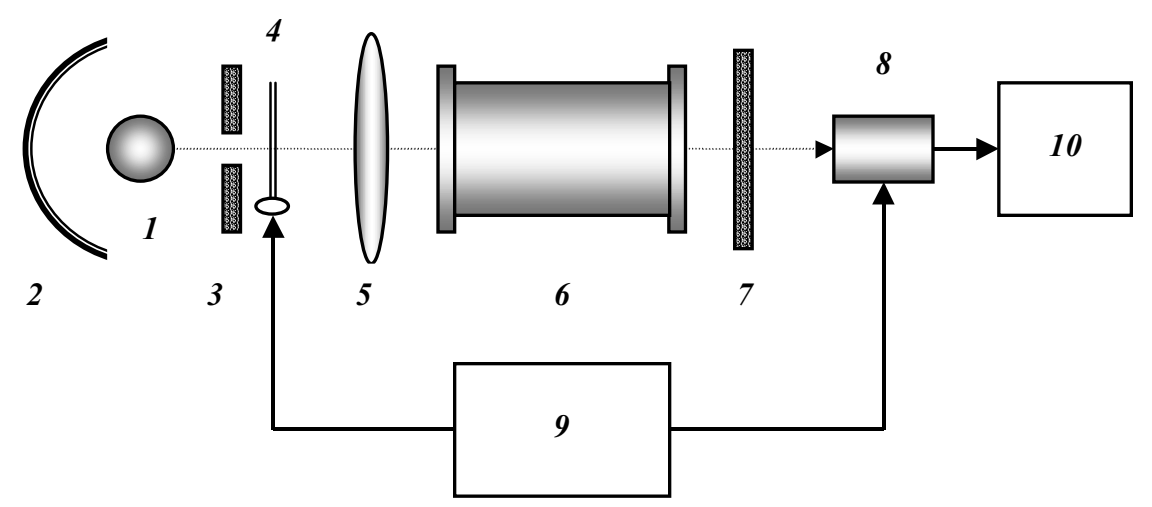

Fig. 4.12 An optical design of infrared spectrophotometer for milk analysis [Posudin and Kostenko, 1994]. 1 - source of infrared radiation, 2 - concave mirror, 3 - diaphragm, 4 modulator of radiation, 5 - lens, 6 - cuvette with a sample, 7 - interference filter, 8 detector, 9 - power supply; 10 - readout system 


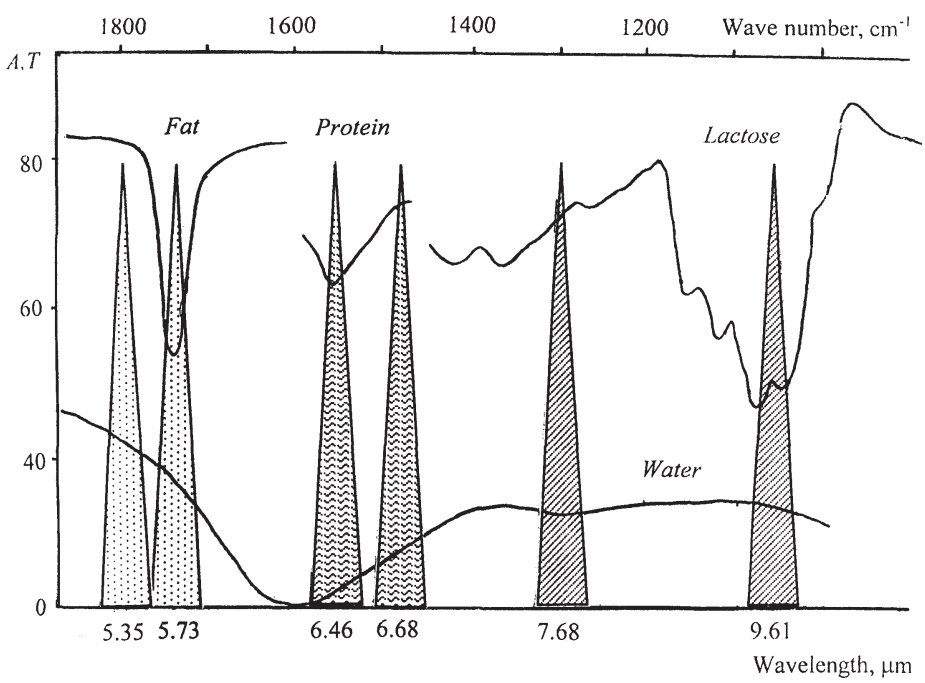

Fig. 4.13 The position of transmission bands of the interference filters which were tuned at the maximum and at the base of absorption band of each component of milk

Table 4.3 Parameters of interference filters which were used in infrared spectrophotometer [Posudin and Kostenko, 1994]

\begin{tabular}{l|c|c|c|c}
\hline $\begin{array}{l}\text { Position Relative to Absorption } \\
\text { Band of Milk Component }\end{array}$ & $\lambda(\mu \mathrm{m})$ & $T(\%)$ & $\Delta_{0.5}(\mu \mathrm{m})$ & $\Delta_{0.1}(\mu \mathrm{m})$ \\
\hline Basis of absorption band of water & $4.50+0.03$ & 43 & 0.11 & 0.42 \\
Maximum of absorption band of water & $4.42-0.05$ & $43-44$ & 0.10 & 0.35 \\
Basis of absorption band of fat & $5.35-0.02$ & $44-45$ & 0.13 & 0.38 \\
Maximum of absorption band of fat & $5.70+0.03$ & 42 & 0.17 & 0.46 \\
Basis of absorption band of protein & $6.68-0.02$ & $40-43$ & 0.22 & 0.56 \\
Maximum of absorption band of protein & $6.46-0.04$ & 40 & 0.18 & 0.57 \\
Basis of absorption band of lactose & $7.68-0.03$ & 50 & 0.23 & 0.54 \\
Maximum of absorption band of lactose & $9.61+0.01$ & 42 & 0.26 & 0.65 \\
\hline
\end{tabular}

If the direction of radiation flow is deviating from normal to the surface of the interference filter, the spectral shift of absorption band and broadening of this band can take place. The value of the shift depends on the angle of incidence and refractive index of filter material. The dependence of refractive index of germanium on the wavelength of radiation can be expressed by the following equation [Voronkova et al., 1965]:

$$
N=A+B L+C L^{2}+D \lambda^{2}+E \lambda^{4}
$$


where $A=3.99931 ; B=0.391707 ; C=0.163492 ; D=0.000006 ; E=$ 0.00000053; $L=\left(\lambda^{2}-0.028\right)^{-1}$.

Thus, refractive index according to this equation is changing from $4.108(\lambda=2 \mu \mathrm{m})$ to $4.005(\lambda=8 \mu \mathrm{m})$.

The values of spectral shift $\Delta \lambda_{\theta} / \lambda_{0}$ can be calculated due the following expression:

$$
\Delta \lambda_{\theta} / \lambda_{0}=\left[\left(n^{2}-\sin ^{2} \theta\right) / n\right]^{1 / 2}
$$

where $\lambda_{0}$-wavelength of incident radiation.

These values are presented in Table 4.4.

Table 4.4 Dependence of spectral shift of absorption band on the deviation of radiation flow direction from normal to the surface of interference filter [Posudin and Kostenko, 1994]

\begin{tabular}{r|c|c}
\hline$\theta^{\circ}$ & $\lambda=2 \mu m(n=4.108)$ & $\lambda=2 \mu m(n=4.024)$ \\
\hline 0 & 1 & 1 \\
5 & 0.99977 & 0.99976 \\
10 & 0.99910 & 0.99907 \\
15 & 0.99800 & 0.99790 \\
20 & 0.99650 & 0.99640 \\
25 & 0.99470 & 0.99450 \\
\hline
\end{tabular}

Readout System. Infrared radiation that was transmitted by the sample entered the pyrometric element PIP with the following characteristics: spectral range $0.5-15 \mu \mathrm{m}$; coefficient of transformation $2 \cdot 10^{3} \mathrm{~V} / \mathrm{W}$; sensitivity threshold $10^{-8}-10^{-9} \mathrm{~W}$; area of sensitive surface $2 \times 2 \mathrm{~mm}^{2}$.

Procedure of Measurement. The concentration of milk component is determined as follows:

$$
C_{i}=K \ln \left(1-\beta_{i}^{\max }+\beta_{i}^{0}\right)
$$

where $K$ - coefficient of proportionality; $\beta_{i}^{\max }$ and $\beta_{i}^{0}$ - values of absorption at the maximum, and at the base of absorption band of $i$ component of milk.

The effect of water absorption is taken into account by the following formula:

$$
\beta_{i}^{\lambda_{i}} \text { (sample) } 100 \%=\left[I_{i}^{\lambda_{i}}\left(\mathrm{H}_{2} \mathrm{O}\right)-I_{i}^{\lambda_{i}}(\text { sample })\right] / I_{i}^{\lambda_{i}}\left(\mathrm{H}_{2} \mathrm{O}\right) \quad \text { (Eqn. 4.4) }
$$

where $I_{i}^{\lambda_{i}}$ (sample) and $I_{i}^{\lambda_{i}}\left(\mathrm{H}_{2} \mathrm{O}\right)$ - intensity of absorption band for milk sample and water at the wavelength $\lambda_{i}$. 


\subsection{NEAR-INFRARED SPECTROSCOPY OF MILK AND MILK COMPONENTS}

The main components of milk (fat, casein, lactose) have specific absorption bands in NIR part of spectrum (Table 4.5).

Table 4.5 Specific absorption bands of milk components in NIR part of spectrum [Fuhrman, 1987]

\begin{tabular}{c|l|c|l}
\hline $\begin{array}{c}\text { Absorption } \\
\text { Maximum }(\mathrm{nm})\end{array}$ & \multicolumn{1}{|c|}{ Component } & $\begin{array}{r}\text { Absorption } \\
\text { Maximum (nm) }\end{array}$ & \multicolumn{1}{c}{ Component } \\
\hline 2,340 & Fat, casein, lactose & 2,100 & Casein, lactose, water \\
2,310 & Fat, casein & 1,340 & Water \\
2,270 & Fat, casein & 1,730 & Fat, casein \\
2,790 & Casein & 1,720 & Fat, casein \\
1,980 & Casein, water & 1,450 & Casein, lactose, water \\
1,820 & Casein, lactose, fat & 1,680 & Casein, water \\
1,780 & Fat, casein & & \\
\hline
\end{tabular}

\subsection{INSTRUMENTATION}

Near-infrared spectrum of milk was investigated with infrared analyzer 4250 "Pacific Scientific". This device has three spectral regions at 1,620$1,800 \mathrm{~nm} ; 1,890-2,115 \mathrm{~nm} ; 2,050-2,320 \mathrm{~nm}$. The following grading equation was used for elaboration of the results of measurements:

$$
Y=B_{0}+B_{1} \cdot O P\left(\lambda_{1}\right)+B_{2} \cdot O P\left(\lambda_{2}\right)+\cdots+B_{N} \cdot O P\left(\lambda_{N}\right)
$$

where $Y$ - result of infrared analysis; $B_{0}, B_{1}, \ldots, B_{N}$ - coefficients of grading equation; $O P\left(\lambda_{i}\right)$ - optical parameters of spectrum at a given wavelength. Optical density $D$ was used as an optical parameter:

$$
D=\lg [1 / R(\lambda)]
$$

where $R(\lambda)$ is diffused reflectance at wavelength $\lambda$.

In addition, the analyzer provides the determination of first $D_{1}$ and second $D_{2}$ derivatives:

$$
\begin{gathered}
D_{1}=\lg [1 / R(\lambda-d \lambda)]-\lg [1 / R(\lambda+d \lambda)] \\
D_{2}=\lg [1 / R(\lambda-d \lambda)]-2 \lg [1 / R(\lambda)]+\lg [1 / R(\lambda+d \lambda)]
\end{gathered}
$$

where $d \lambda$ is a step of derivative. 


\subsection{RESULTS OF NEAR-INFRARED SPECTROSCOPY OF MILK}

The main objective of this investigation was to determine the principal spectral characteristics of milk samples with different contents of components, to estimate level of correlation between these components, and to compare the results of determination of milk components which were obtained due to NIR method and traditional chemical methods.

The following coefficient of correlation $R$ was used for comparison of the results of measurements by NIR $\left(Y_{\text {NIR }}\right)$ and chemical $\left(Y_{\text {chem }}\right)$ methods:

$$
R=\frac{N \sum\left(Y_{\text {chem }} \cdot Y_{\text {NIR }}\right)-\sum\left(Y_{\text {chem }} \cdot Y_{\text {NIR }}\right)}{\left[N \sum Y_{\text {chem }}^{2}-\left(\sum Y_{\text {chem }}\right)^{2}\right]^{1 / 2} \times\left[N \sum Y_{N I R}^{2}-\left(\sum Y_{N I R}\right)^{2}\right]^{1 / 2}}
$$

(Eqn. 4.9)

The results of chemical and NIR analysis of 50 samples of milk are given in Table 4.6 and the results of calculation of coefficients of grading equation (Eqn. 4.5 ) are presented in Table 4.7.

The correlation dependence between results of chemical and NIR analysis of milk components are presented in Fig. 4.14 for fat, Fig. 4.15 for protein, Fig. 4.16 for non-fat solids and Fig. 4.17 for total solids.

The values of coefficients of correlation which were obtained during analysis of 50 samples of milk are given in Table 4.8 .

Near-infrared spectra of milk samples with different contents of fat $(1.82 \% ; 4.27 \% ; 4.48 \% ; 4.73 \% ; 7.69 \%)$ are presented in Fig. 4.18 . It is clear that the amplitude of these spectra depends strongly on the fat content. It was also interesting to compare the NIR spectra of the samples that had the same fat (Fig. 4.19) and protein (Fig. 4.20) concentration according to the chemical testing: the results demonstrate some divergence which are caused by inaccuracy of the chemical method. The derivative spectra are rather informative - they make it possible to find extreme points of the reflectance spectra (samples N 1, 5 and 7) (Fig. 4.21).

The next step of investigation was the clearing up of the level of correlation between separate components of milk which were determined by chemical and NIR methods. The correlation dependence between the main components of milk (fat, protein, total solids, and nonfat solids) are presented in Figs. 4.22-4.27. The highest level of correlation was indicated between the content of fat and total solids that was determined by NIR method (Fig. 4.27). The values of coefficients of correlation are given in Table 4.9. 
Table 4.6 Results of chemical and infrared analysis

\begin{tabular}{|c|c|c|c|c|c|c|c|c|c|}
\hline $\begin{array}{l}\text { Sample } \\
\text { number }\end{array}$ & Fat & Protein & $\begin{array}{l}\text { Dry } \\
\text { residue }\end{array}$ & $\begin{array}{c}\text { Dry } \\
\text { substance }\end{array}$ & $\begin{array}{l}\text { Sample } \\
\text { number }\end{array}$ & Fat & Protein & $\begin{array}{c}\text { Dry } \\
\text { residue }\end{array}$ & $\begin{array}{c}\text { Dry } \\
\text { substance }\end{array}$ \\
\hline 1 & 8.40 & 2.90 & 8.20 & 16.60 & 1 & 7.69 & 3.44 & 8.76 & 16.68 \\
\hline 2 & 4.40 & 3.70 & 8.60 & 13.00 & 2 & 4.73 & 3.02 & 8.25 & 12.92 \\
\hline 3 & 4.10 & 2.90 & 8.30 & 12.40 & 3 & 4.27 & 2.67 & 7.98 & 12.04 \\
\hline 4 & 4.50 & 2.50 & 7.90 & 12.40 & 4 & 4.48 & 2.65 & 8.00 & 12.32 \\
\hline 5 & 1.40 & 3.80 & 9.10 & 10.50 & 5 & 1.82 & 3.30 & 8.46 & 9.91 \\
\hline 6 & 3.80 & 2.70 & 8.00 & 11.80 & 6 & 3.86 & 2.76 & 8.16 & 12.14 \\
\hline 7 & 4.40 & 3.50 & 8.70 & 13.10 & 7 & 4.45 & 2.61 & 8.80 & 12.25 \\
\hline 8 & 5.40 & 3.20 & 8.70 & 14.10 & 8 & 5.36 & 2.95 & 8.39 & 13.44 \\
\hline 9 & 4.00 & 2.70 & 8.00 & 12.00 & 9 & 4.14 & 2.66 & 8.17 & 12.24 \\
\hline 10 & 5.00 & 3.00 & 8.20 & 13.20 & 10 & 5.05 & 2.88 & 8.26 & 13.29 \\
\hline 11 & 2.10 & 2.30 & 7.50 & 9.60 & 11 & 2.03 & 2.99 & 7.98 & 10.07 \\
\hline 12 & 2.65 & 2.80 & 8.00 & 10.65 & 12 & 2.44 & 2.80 & 7.99 & 10.40 \\
\hline 13 & 3.90 & 2.40 & 7.50 & 11.40 & 13 & 3.74 & 2.58 & 7.97 & 11.75 \\
\hline 14 & 3.90 & 3.20 & 8.60 & 12.50 & 14 & 3.99 & 2.63 & 8.19 & 11.87 \\
\hline 15 & 3.50 & 2.70 & 7.50 & 11.00 & 15 & 3.60 & 2.71 & 8.23 & 11.53 \\
\hline 16 & 4.50 & 2.70 & 7.60 & 11.10 & 16 & 4.43 & 2.88 & 7.93 & 12.35 \\
\hline 17 & 5.70 & 3.00 & 8.30 & 14.00 & 17 & 4.91 & 2.87 & 8.14 & 13.34 \\
\hline 18 & 4.10 & 3.40 & 8.70 & 12.80 & 18 & 4.14 & 2.69 & 8.10 & 11.83 \\
\hline 19 & 3.30 & 2.80 & 7.90 & 11.20 & 19 & 3.70 & 3.37 & 8.38 & 11.18 \\
\hline 20 & 3.80 & 3.00 & 8.30 & 12.10 & 20 & 3.86 & 3.01 & 8.33 & 12.40 \\
\hline 21 & 5.00 & 3.20 & 8.70 & 13.70 & 21 & 5.20 & 2.75 & 8.32 & 13.56 \\
\hline 22 & 3.50 & 2.60 & 8.10 & 11.60 & 22 & 4.33 & 2.61 & 7.81 & 11.67 \\
\hline 23 & 4.00 & 2.80 & 8.10 & 12.10 & 23 & 4.14 & 2.75 & 8.36 & 12.31 \\
\hline 24 & 3.10 & 2.80 & 8.00 & 11.10 & 24 & 3.09 & 2.78 & 8.18 & 11.40 \\
\hline 25 & 4.00 & 2.00 & 7.30 & 11.30 & 25 & 3.94 & 2.47 & 7.84 & 11.57 \\
\hline 26 & 3.30 & 2.60 & 7.50 & 10.80 & 26 & 2.85 & 2.59 & 7.48 & 10.76 \\
\hline 27 & 3.70 & 2.45 & 7.40 & 11.10 & 27 & 3.52 & 2.61 & 7.79 & 11.32 \\
\hline 28 & 3.20 & 2.10 & 7.20 & 10.40 & 28 & 3.03 & 2.50 & 7.52 & 10.46 \\
\hline 29 & 4.70 & 3.10 & 8.40 & 13.10 & 29 & 4.69 & 2.87 & 7.89 & 12.93 \\
\hline 30 & 2.70 & 2.70 & 7.90 & 10.60 & 30 & 2.72 & 2.78 & 7.76 & 10.63 \\
\hline 31 & 4.00 & 2.90 & 8.30 & 12.30 & 31 & 4.06 & 2.70 & 7.88 & 12.01 \\
\hline 32 & 3.90 & 3.00 & 8.20 & 12.10 & 32 & 3.80 & 2.92 & 7.83 & 12.18 \\
\hline 33 & 4.10 & 2.25 & 7.30 & 11.40 & 33 & 4.85 & 2.57 & 7.53 & 12.46 \\
\hline 34 & 3.40 & 2.50 & 7.60 & 11.00 & 34 & 3.36 & 2.95 & 8.22 & 11.76 \\
\hline 35 & 3.30 & 2.50 & 7.60 & 10.90 & 35 & 3.26 & 2.50 & 7.66 & 11.30 \\
\hline 36 & 3.90 & 2.90 & 8.30 & 12.20 & 36 & 3.90 & 2.96 & 8.03 & 12.39 \\
\hline 37 & 3.20 & 1.20 & 7.50 & 10.70 & 37 & 3.15 & 2.51 & 7.62 & 10.94 \\
\hline 38 & 3.90 & 2.50 & 8.40 & 12.30 & 38 & 3.80 & 2.57 & 7.79 & 11.38 \\
\hline 39 & 4.60 & 2.40 & 7.60 & 12.20 & 39 & 4.59 & 2.43 & 7.66 & 11.96 \\
\hline
\end{tabular}




\begin{tabular}{|c|c|c|c|c|c|c|c|c|c|}
\hline 40 & 4.90 & 3.10 & 8.00 & 12.90 & 40 & 4.89 & 2.62 & 7.76 & 12.58 \\
\hline 41 & 3.90 & 2.60 & 7.70 & 11.60 & 41 & 3.85 & 2.52 & 7.68 & 11.37 \\
\hline 42 & 3.20 & 2.65 & 7.80 & 11.00 & 42 & 3.16 & 2.64 & 7.60 & 10.79 \\
\hline 43 & 4.60 & 2.90 & 8.20 & 12.80 & 43 & 4.65 & 2.94 & 7.78 & 12.55 \\
\hline 44 & 2.80 & 2.20 & 7.30 & 10.10 & 44 & 2.52 & 2.52 & 1.58 & 10.18 \\
\hline 45 & 4.60 & 2.35 & 7.40 & 12.00 & 45 & 4.36 & 2.65 & 7.75 & 12.12 \\
\hline 46 & 3.50 & 2.90 & 7.70 & 11.20 & 46 & 3.20 & 2.70 & 7.73 & 11.51 \\
\hline 47 & 5.00 & 2.80 & 7.30 & 12.30 & 47 & 5.62 & 2.72 & 7.52 & 13.33 \\
\hline 48 & 4.10 & 3.20 & 8.00 & 12.10 & 48 & 3.96 & 2.77 & 7.77 & 12.06 \\
\hline 49 & 4.70 & 2.60 & 7.60 & 12.30 & 49 & 4.50 & 2.58 & 7.64 & 11.95 \\
\hline 50 & 4.00 & 2.70 & 7.50 & 11.50 & 50 & 3.96 & 2.71 & 7.77 & 11.78 \\
\hline
\end{tabular}

Table 4.7 Results of calculation of coefficients of grading equation (Eqn. 4.5 ) [Posudin and Kostenko, 1994]

\begin{tabular}{|c|c|c|c|}
\hline $\begin{array}{l}\text { Coefficient of } \\
\text { Regression Equation }\end{array}$ & $\begin{array}{l}\text { F-criterion for } \\
\text { Given Equation }\end{array}$ & $\begin{array}{c}\text { Scale } \\
\text { Division }\end{array}$ & $\begin{array}{l}\text { Wave Number, } \\
\qquad \mathrm{cm}^{-1}\end{array}$ \\
\hline \multicolumn{4}{|c|}{ Protein } \\
\hline \multicolumn{4}{|l|}{$\mathrm{B}(0)=8.719$} \\
\hline$B(1)=64.680$ & 14.02 & 285 & 2,197 \\
\hline$B(2)=230.190$ & 14.79 & 93 & 1,792 \\
\hline$B(3)=-69.974$ & 19.95 & 35 & 1,705 \\
\hline$B(4)=-73.980$ & 62.69 & 264 & 2,131 \\
\hline \multicolumn{4}{|c|}{ Fat } \\
\hline \multicolumn{4}{|l|}{$\mathrm{B}(0)=6.918$} \\
\hline$B(1)=316.151$ & 44.84 & 88 & 1,789 \\
\hline$B(2)=-108.606$ & 506.08 & 24 & 1,679 \\
\hline \multicolumn{4}{|c|}{ Non-fat Solids } \\
\hline \multicolumn{4}{|l|}{$\mathrm{B}(0)=14.539$} \\
\hline$B(1)=-36.935$ & 58.48 & 186 & 2,062 \\
\hline$B(2)=-82.348$ & 77.57 & 24 & 1,679 \\
\hline \multicolumn{4}{|c|}{ Total Solids } \\
\hline \multicolumn{4}{|l|}{$\mathrm{B}(0)=21.224$} \\
\hline $\mathrm{B}(1)=-81.700$ & 35.03 & 271 & 2,154 \\
\hline$B(2)=135.838$ & 11.23 & 293 & 2,219 \\
\hline$B(3)=243.213$ & 21.40 & 350 & 2,315 \\
\hline$B(4)=-172.508$ & 66.10 & 28 & 1,688 \\
\hline
\end{tabular}

NIR method provides the analysis of milk samples which contain a high proportion of water and demonstrate high level of opacity. The NIR method is rapid, non-destructive and offers a high level of accuracy in comparison with traditional chemical methods. 


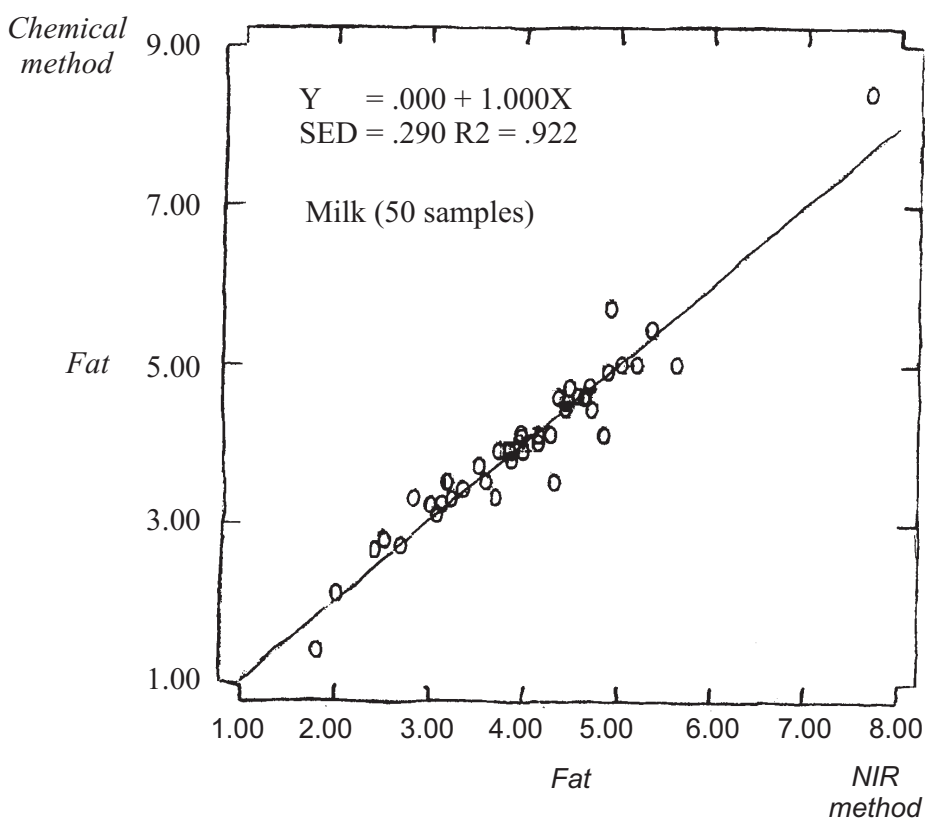

Fig. 4.14 The correlation dependence between results of chemical and NIR analysis of fat in milk

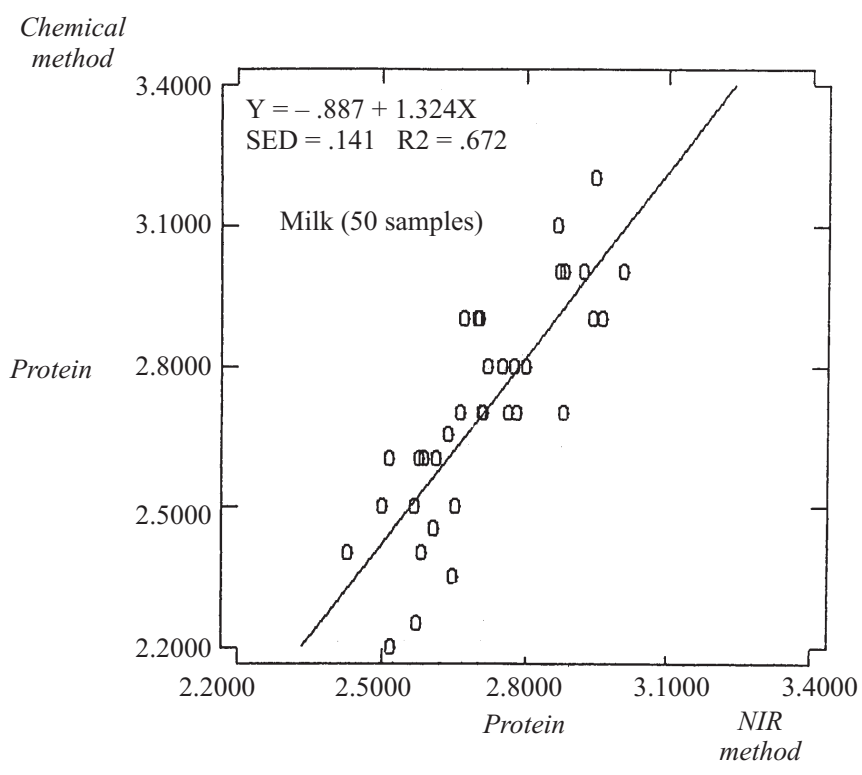

Fig. 4.15 The correlation dependence between results of chemical and NIR analysis of protein in milk 


\section{Practical Spectroscopy in Agriculture and Food Science}

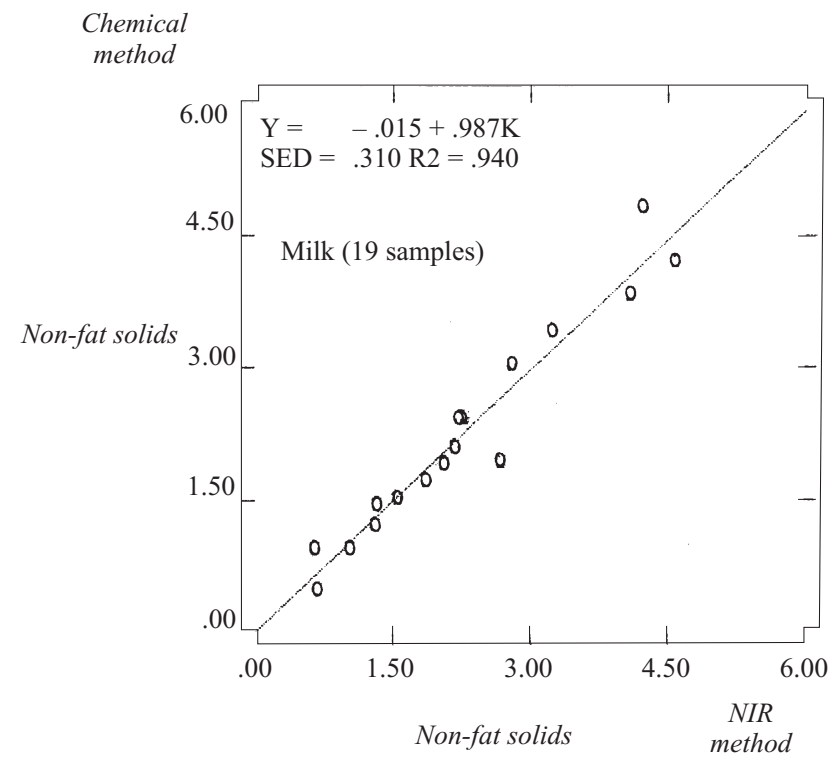

Fig. 4.16 The correlation dependence between results of chemical and NIR analysis of non-fat solids in milk

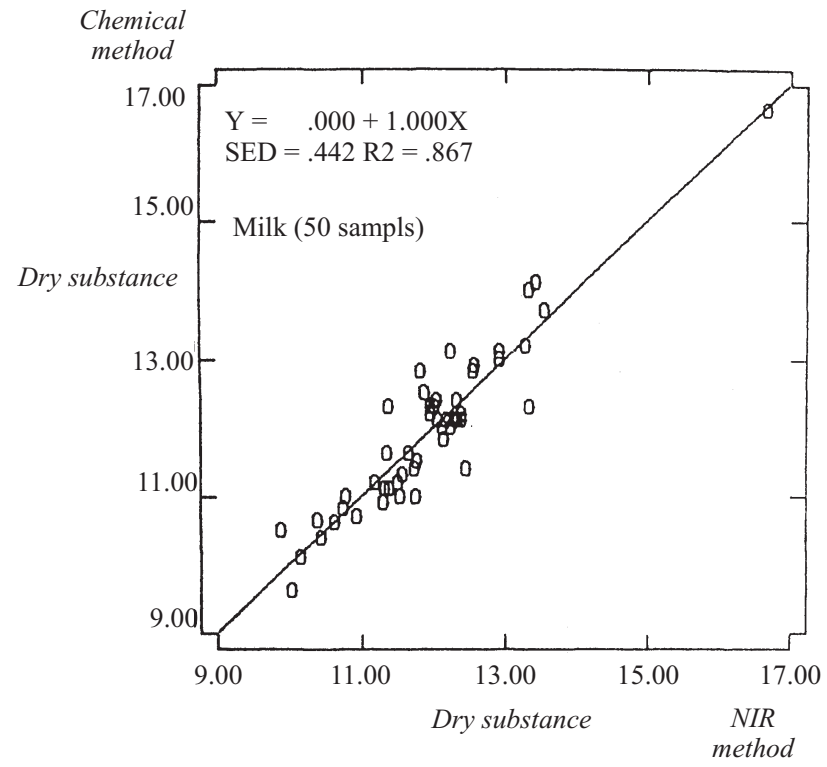

Fig. 4.17 The correlation dependence between results of chemical and NIR analysis of total solids in milk 
Table 4.8 Values of coefficients of correlation dependence between results of chemical and NIR analysis of milk components [Posudin and Kostenko, 1994]

\begin{tabular}{l|l|c|c|c|c|c|c}
\hline $\begin{array}{c}\text { Type of } \\
\text { Analysis }\end{array}$ & Component & $\begin{array}{c}\text { Mean } \\
\text { Content (\%) }\end{array}$ & Deviation & SEP & SEP(C) & $R$ & $R_{2}$ \\
\hline Chemical & Fat & 3.99 & 1.028 & 0.284 & 0.287 & 0.960 & 0.922 \\
NIR & Fat & 3.99 & 0.987 & & & & \\
Chemical & Protein & 2.75 & 0.439 & 0.375 & 0.379 & 0.504 & 0.254 \\
NIR & Protein & 2.75 & 0.221 & & & & \\
Chemical & Non-fat Solids & 7.95 & 0.465 & 0.357 & 0.361 & 0.630 & 0.397 \\
NIR & Non-fat Solids & 7.95 & 0.293 & & & & \\
Chemical & Total Solids & 11.94 & 1.198 & 0.433 & 0.437 & 0.931 & 0.867 \\
NIR & Total Solids & 11.94 & 1.115 & & & & \\
\hline
\end{tabular}

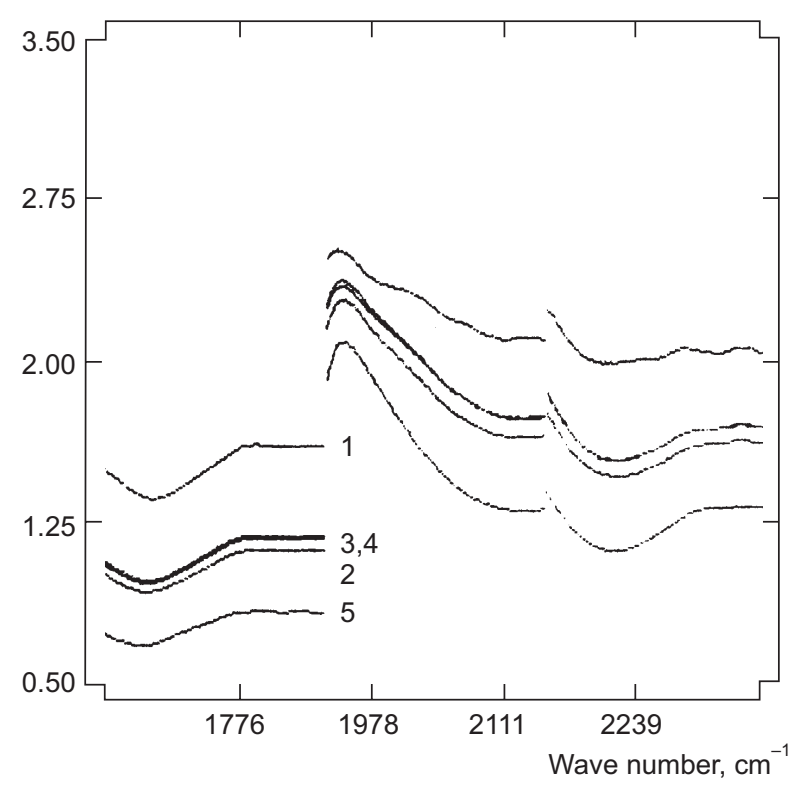

Fig. 4.18 The near-infrared spectra of milk samples with different contents of fat (1 $1.82 \%$; $2-4.27 \%$; $3-4.48 \% ; 4-4.73 \% ; 5-7.69 \%$ )

\subsection{FLUORESCENCE SPECTROSCOPY OF MILK AND MILK COMPONENTS}

Milk contains a number of fluorophors - compounds which can produce fluorescence. The wavelengths of excitation and emission of fluorescence of the main milk fluorophores at room temperature are presented in 
48 Practical Spectroscopy in Agriculture and Food Science

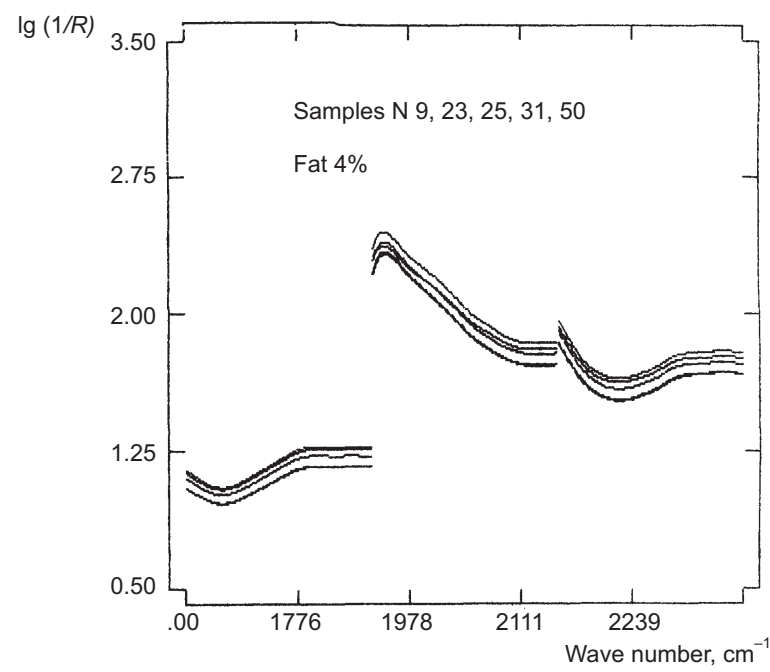

Fig. 4.19 The near-infrared spectra of the samples that had the same fat concentration according to the chemical testing

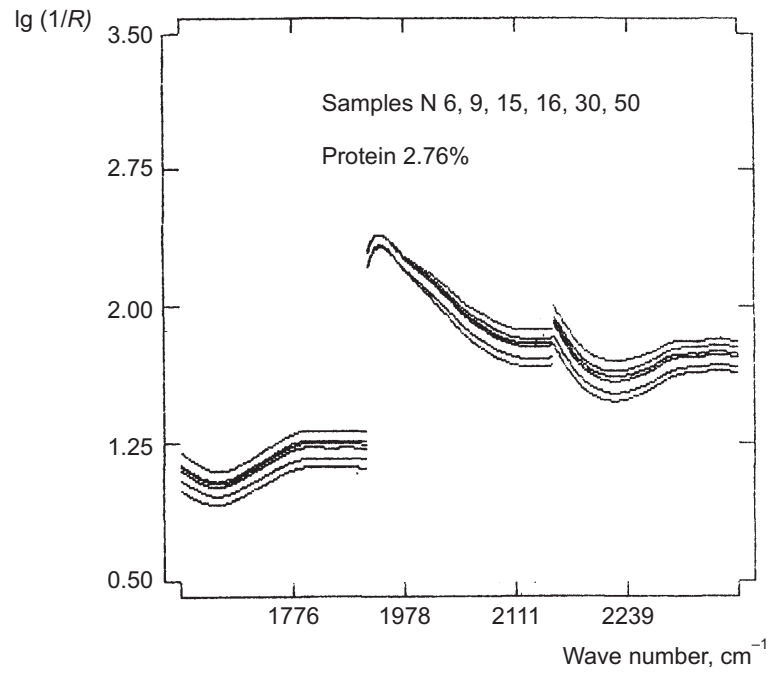

Fig. 4.20 The near-infrared spectra of the samples that had the same protein concentration according to the chemical testing

Table 4.10. The fluorescence properties of amino acids and proteins depend on the solvent, the substituent groups, $\mathrm{pH}$ of the medium and the temperature. The process of oxidation and photodegradation cause 


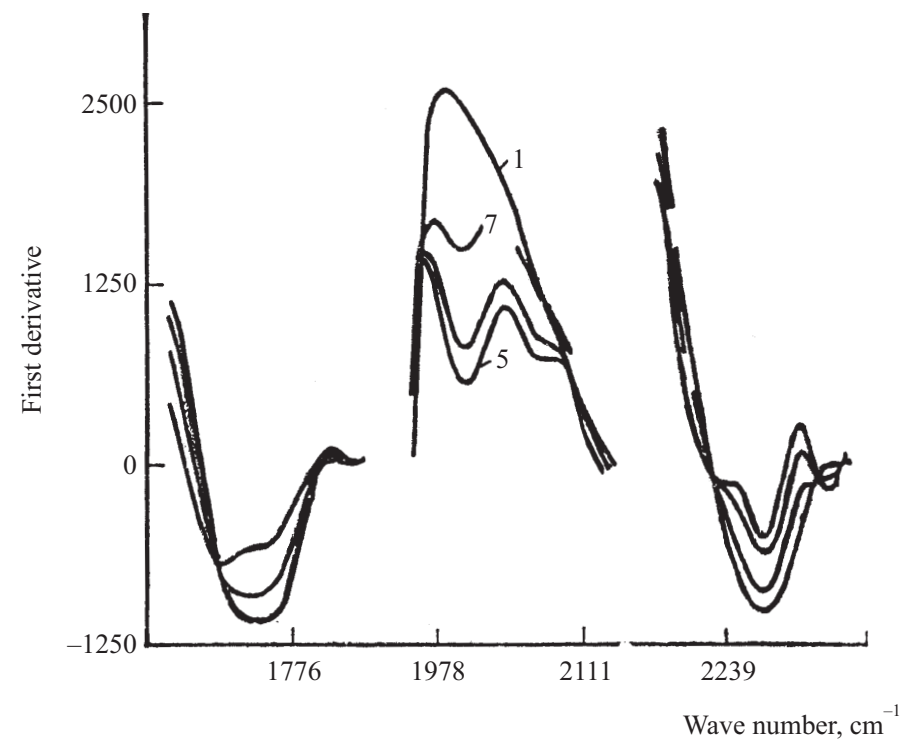

Fig. 4.21 The derivative spectra of milk (samples N 1, 5 and 7)

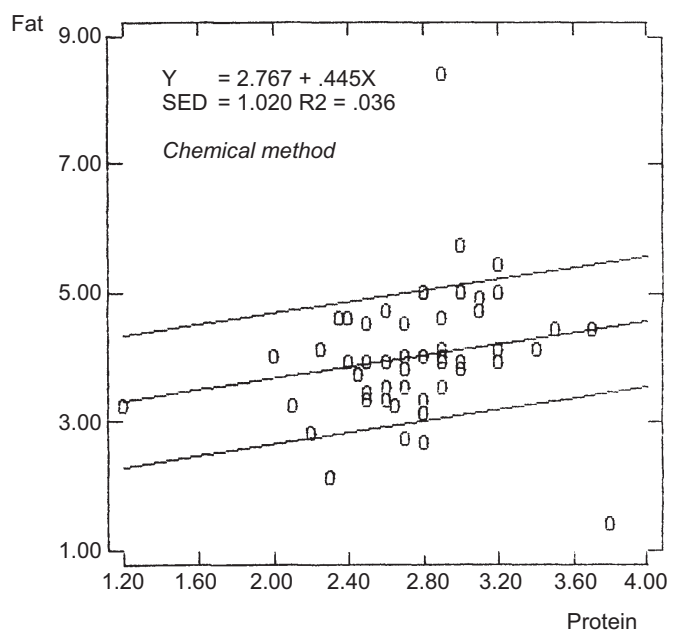

Fig. 4.22 The correlation between fat and protein content of milk which was determined by chemical method

the change in fluorescence intensity and spectral shifts in fatty acids. The fluorescence of soluble vitamins $\left(B_{1}, B_{2}, B_{12}, P P, H\right)$ depends on the temperature, solvent and $\mathrm{pH}$ of the medium. 
50 Practical Spectroscopy in Agriculture and Food Science

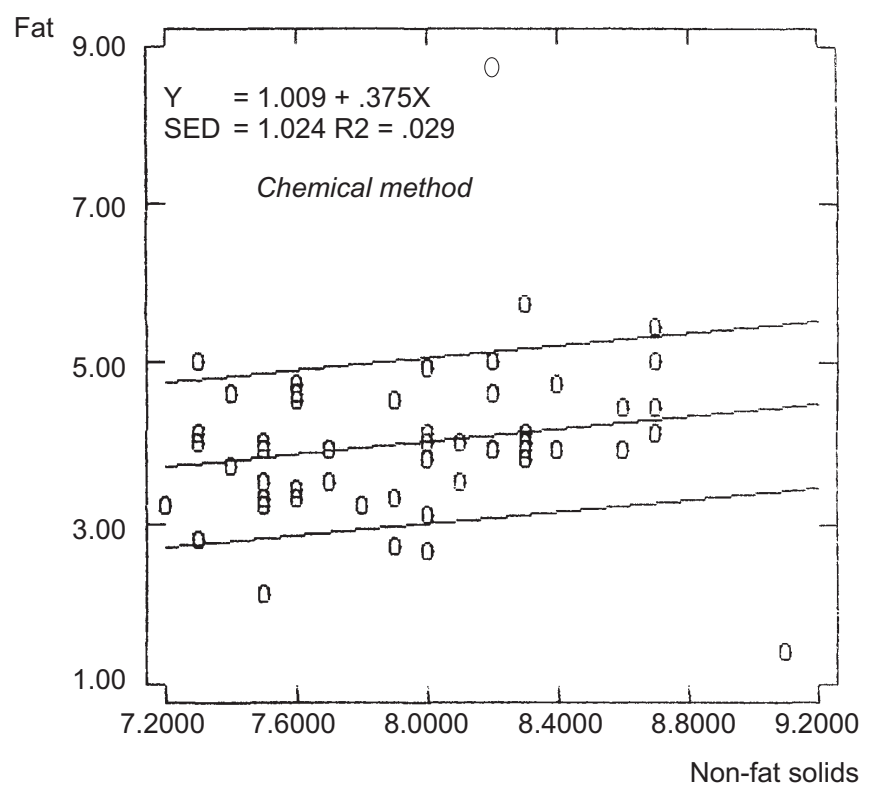

Fig. 4.23 The correlation between fat and non-fat solids content of milk which was determined by chemical method

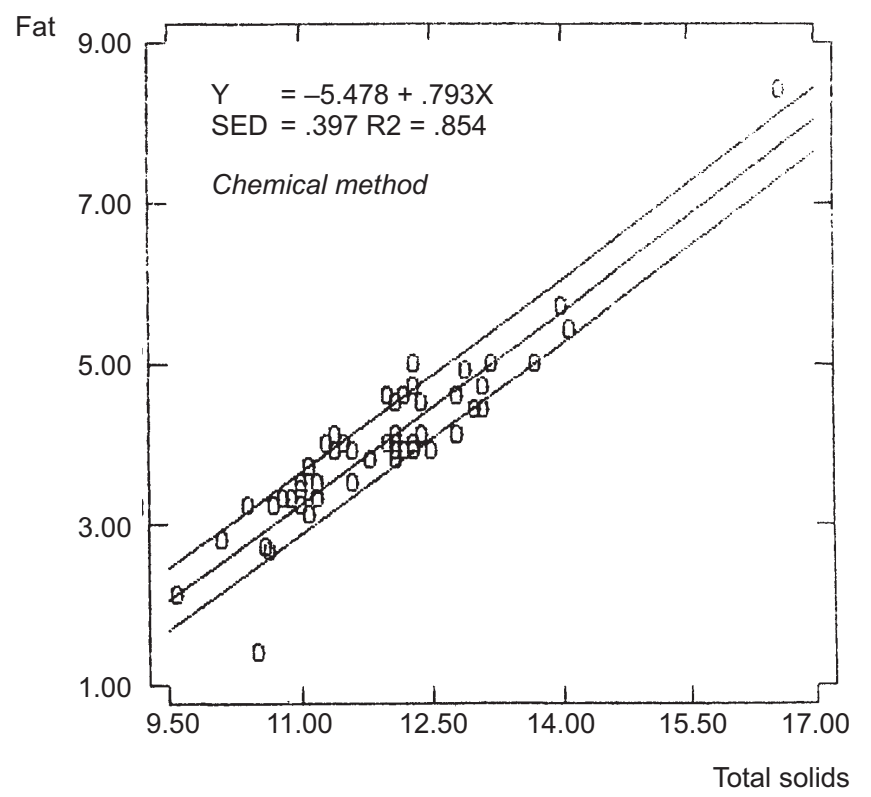

Fig. 4.24 The correlation between fat and total solids content of milk which was determined by chemical method 


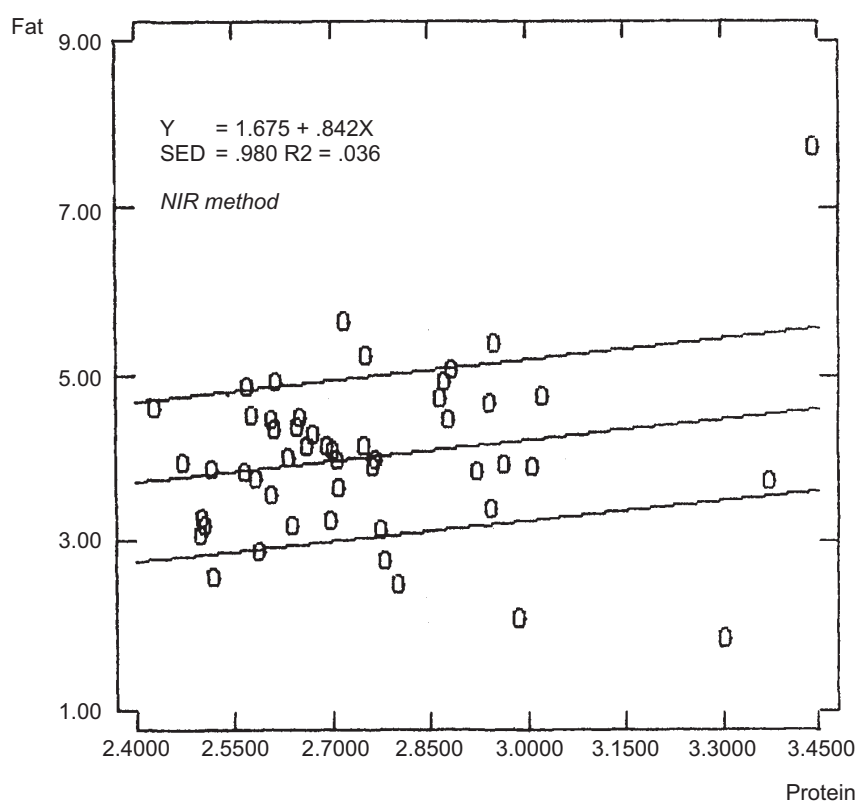

Fig. 4.25 The correlation between fat and protein content of milk which was determined by NIR method

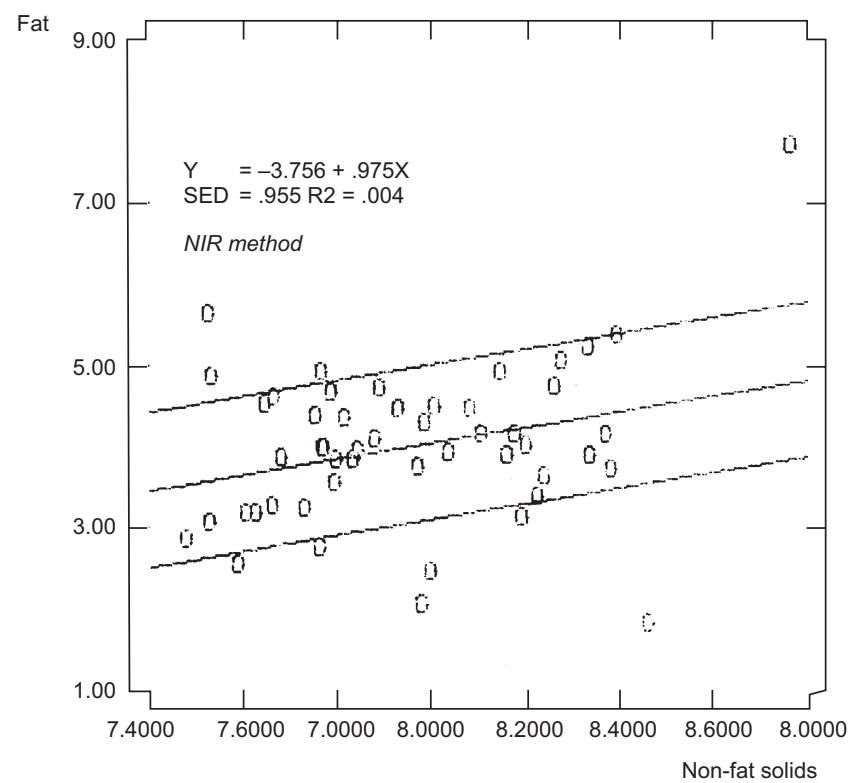

Fig. 4.26 The correlation between fat and non-fat solids content of milk which was determined by NIR method 


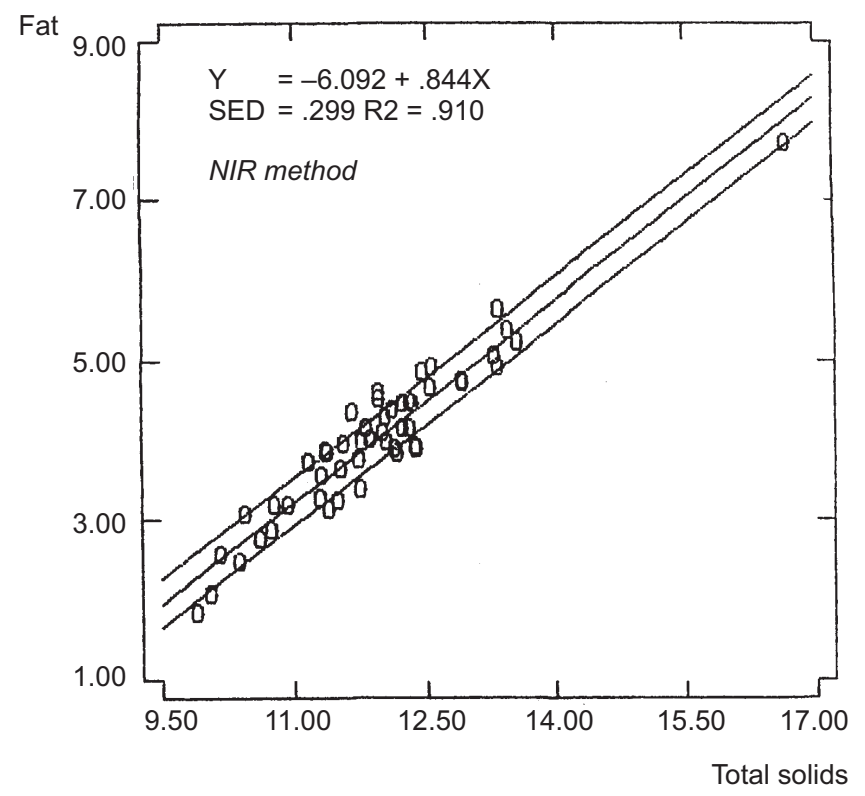

Fig. 4.27 The correlation between fat and total solids content of milk which was determined by NIR method

Table 4.9 Values of coefficients of correlation between separate components of milk which were determined by chemical and NIR methods [Posudin and Kostenko, 1994]

\begin{tabular}{l|c|c|c|c}
\hline Component & Fat & Protein & Non-fat Solids & Total Solids \\
\hline Fat & - & 0.036 & 0.084 & 0.910 \\
Protein & 0.036 & - & 0.519 & 0.123 \\
Non-fat Solids & 0.084 & 0.519 & - & 0.200 \\
Total Solids & 0.910 & 0.123 & 0.200 & - \\
\hline
\end{tabular}

\subsection{INSTRUMENTATION}

Fluorescence properties of milk and its components were measured by spectrophotometer SDL-2 at room temperature in spectral range 280-700 $\mathrm{nm}$. Xenon lamp DKeSh-150 was used as a source of fluorescence excitation. The fluorescence emission radiation was registered at the angle $90^{\circ}$ to the direction of excitation radiation. The photomultiplier FEU-100 was used for the detection of fluorescence emission. The absolute error of the determination of spectral line position was $\pm 2 \mathrm{~nm}$ 
Table 4.10 Dependence of fluorescence emission of milk components on the excitation wavelength

\begin{tabular}{c|c|l}
\hline $\begin{array}{c}\text { Wavelength of } \\
\text { Excitation }(\mathrm{nm})\end{array}$ & $\begin{array}{c}\text { Maximum of } \\
\text { Emission }(\mathrm{nm})\end{array}$ & $\begin{array}{c}\text { Component (Responsible } \\
\text { for Fluorescence) }\end{array}$ \\
\hline $270-290$ & 340 & Amino acids, proteins \\
& 370 & Folic acid \\
& 340 & Vitamin $E$ \\
\hline $300-310$ & 340 & Proteins (casein) \\
& 520 & Riboflavin \\
\hline $315-320$ & 398 & Vitamin $B_{12}$ \\
& 520 & Riboflavin \\
& 400 & Fat acids \\
\hline $320-340$ & 400 & Vitamin $B_{6}$ \\
& 400 & Vitamin $B_{12}$ \\
& 510 & Vitamin $A$ \\
& 520 & Riboflavin \\
\hline $340-370$ & 424 & Linetol \\
& 450 & Thiochrome $\left(B_{1}\right)$ \\
& 450 & Folic acid \\
& 460 & Vitamin $C$ \\
& 460 & Vitamin $B_{3}$ \\
& 471 & Vitamin $B_{6}$ \\
& 480 & Vitamin $D$ \\
& 520 & Riboflavin \\
\hline 400 & 520 & Riboflavin \\
\hline & &
\end{tabular}

and of half-width of spectral line was not more than $\pm 5 \mathrm{~nm}$. The relative error of determination of the intensity of spectral lines did not exceed $\pm 2 \%$.

\subsection{RESULTS OF FLUORESCENCE SPECTROSCOPY OF MILK}

\subsubsection{Whole Milk}

The problem of determination of proteins and fat in milk can be resolved by the selection of wavelength of fluorescence excitation. The typical spectra of fluorescence emission of milk for different wavelengths of excitation (Figs. $4.28 a$ and $b$ ) demonstrate that the selection of proteins or fat can be achieved by the choice of excitation at 280 or $325 \mathrm{~nm}$ correspondingly. The emission of fluorescence can be analyzed at 330$340 \mathrm{~nm}$ for proteins and at $400-410 \mathrm{~nm}$ for fat. 


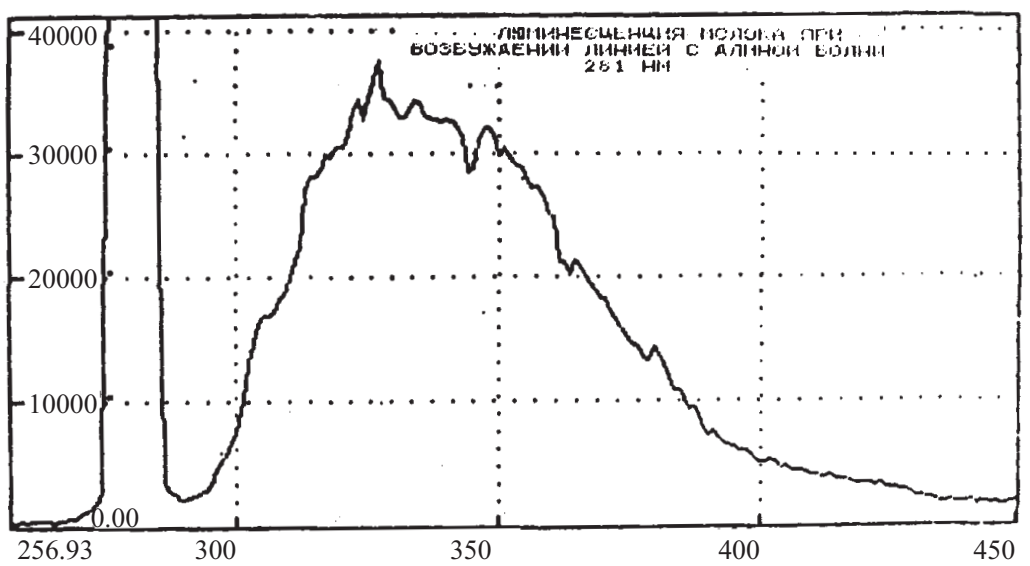

(a)

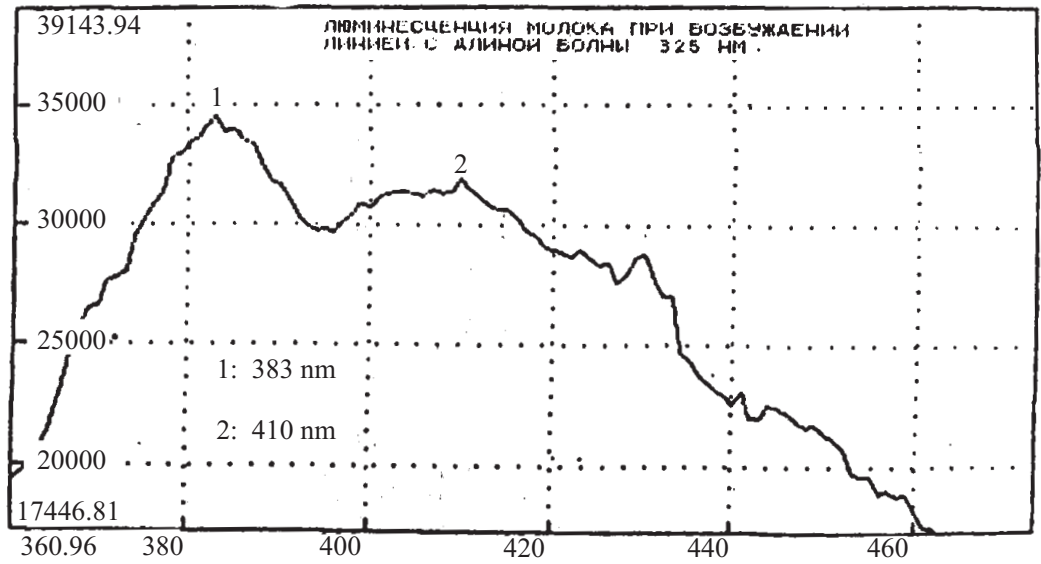

(b)

Fig. 4.28 The typical spectrum of fluorescence emission of milk: $a$ - wavelength of excitation $281 \mathrm{~nm} ; b$ - wavelength of excitation $325 \mathrm{~nm}$

\subsubsection{Proteins}

Aromatic Amino Acids. The main amino acids of milk such as tryptophan, tyrosine, and phenil alanine, demonstrate fluorescence properties. Fluorescence emission spectrum of tryptophan (Fig. 4.29) was measured by us; it is characterized by maxima at $289 \mathrm{~nm}, 308 \mathrm{~nm} ; 334 \mathrm{~nm}$ and 360 $\mathrm{nm}$ (excitation wavelength is $280 \mathrm{~nm}$ ). Tyrosine in aqueous solutions demonstrates at room temperature fluorescence band of half-width of about $34 \mathrm{~nm}$ at $303-304 \mathrm{~nm}$ (excitation wavelength is $275 \mathrm{~nm}$ ); 


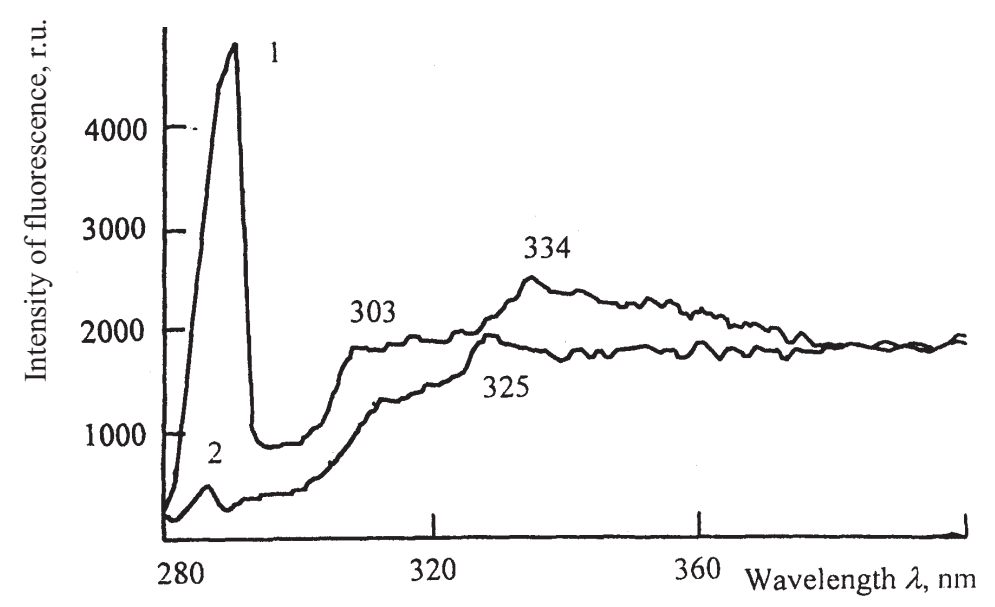

Fig. 4.29 Fluorescence emission spectrum of tryptophan (excitation wavelength is 280 $\mathrm{nm})$. Wavelength of excitation: $1-280 \mathrm{~nm} ; 2-313 \mathrm{~nm}$

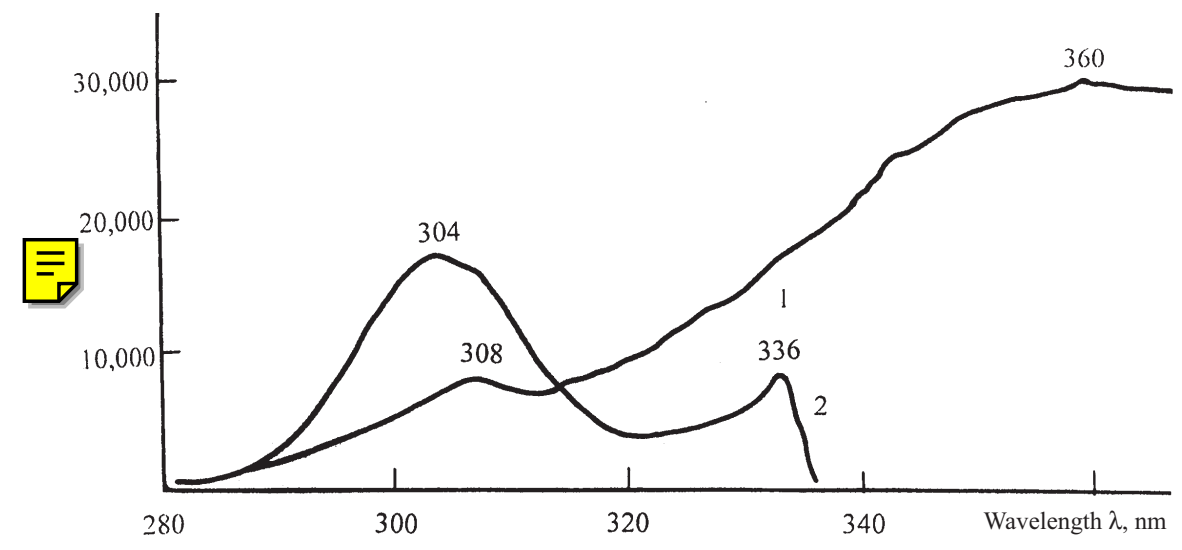

Fig. 4.30 Fluorescence excitation spectra of casein (emission wavelength: $1-350 \mathrm{~nm}$; $2-400 \mathrm{~nm})$

phenylalanine has fluorescence emission maxima at $275 \mathrm{~nm}, 282 \mathrm{~nm}$ and $289 \mathrm{~nm}$ [Brusilovsky and Waynberg, 1990].

Casein. This component constitutes about $83 \%$ of all protein components of milk. Excitation fluorescence spectra of casein are given in Fig. 4.30; maxima of excitation spectrum are located at $304 \mathrm{~nm}$ and 336 $\mathrm{nm}$ (emission wavelength is $350 \mathrm{~nm}$ ); fluorescence emission spectra of casein (Fig. 4.31) have maxima at 334-341 nm (excitation wavelengths are $290 \mathrm{~nm}, 295 \mathrm{~nm} ; 300 \mathrm{~nm}$; and $305 \mathrm{~nm})$. 


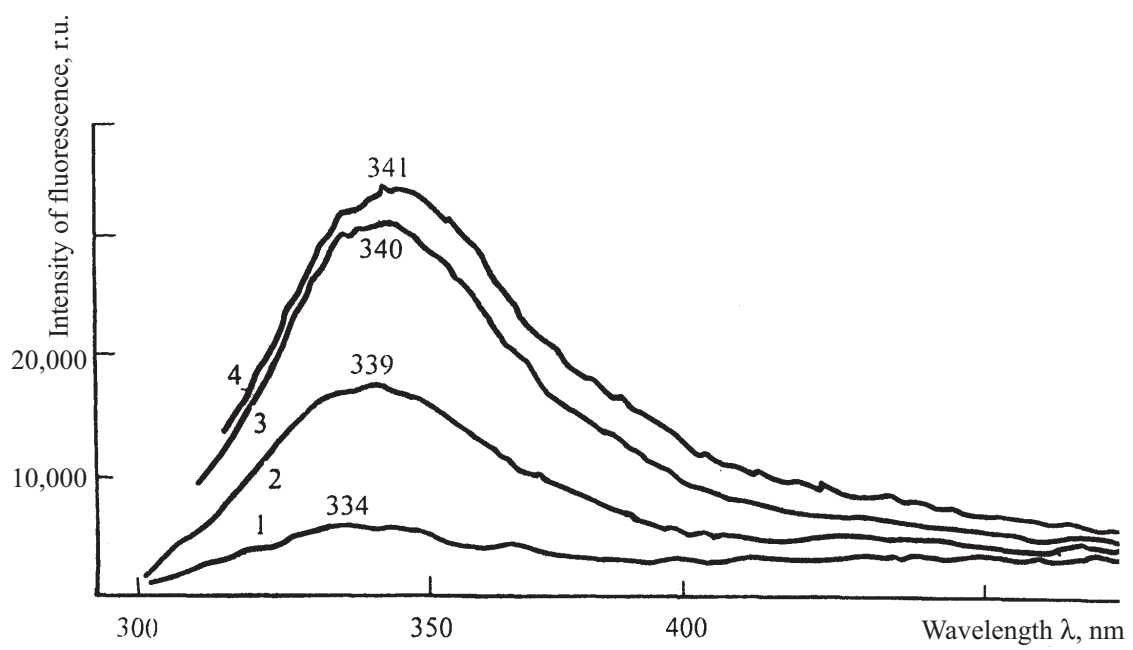

Fig. 4.31 Fluorescence emission spectra of casein (excitation wavelengths: $1-290 \mathrm{~nm}$, 2 - $295 \mathrm{~nm} ; 3-300 \mathrm{~nm} ; 4-305 \mathrm{~nm}$ )

\subsubsection{Fat Acids}

Milk fat consists of polyunsaturated acids - linoleic acid with two unsaturated bonds, linolenic acid with three double bonds, and arachidonic acid with four double bonds. We have used linetol (mixture of these three acids) as a model system in our investigations. Fluorescence excitation spectrum of linetol is characterized by maximum at $362 \mathrm{~nm}$ with half-width of about $30 \mathrm{~nm}$ (Fig. 4.32); fluorescence emission spectrum has a maximum at $424 \mathrm{~nm}$ (Fig. 4.33) with half-width of about $100 \mathrm{~nm}$. A strong effect of oxidation on the intensity of fluorescence of linetol - 7\% decreasing during 10 minutes, was observed in our experiments.

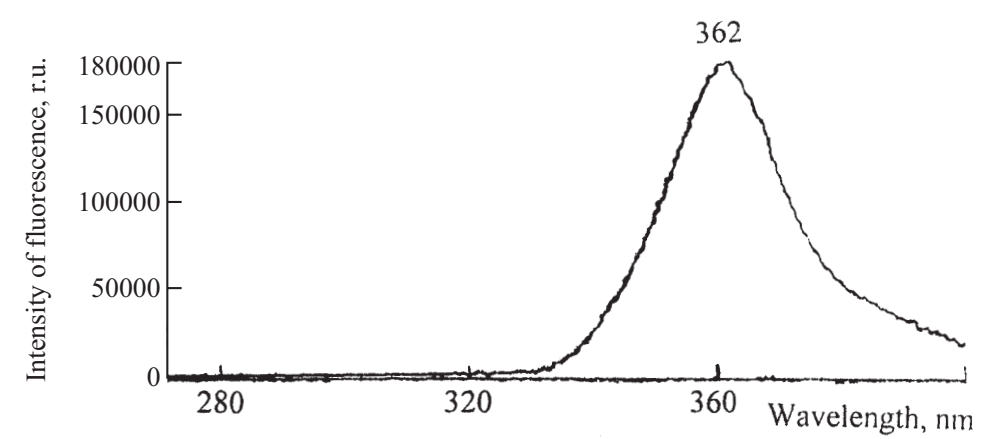

Fig. 4.32 Fluorescence excitation spectrum of linetol (emission wavelength is $425 \mathrm{~nm}$ ) 


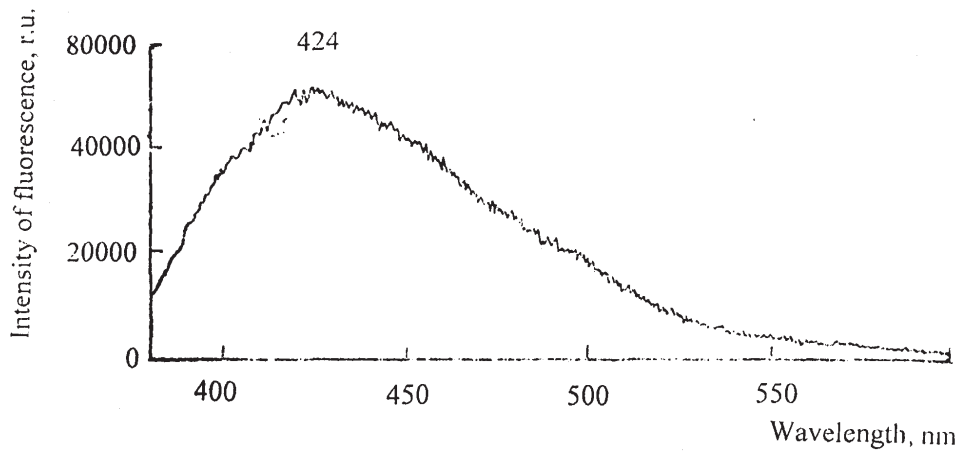

Fig. 4.33 Fluorescence emission spectrum of linetol (excitation wavelength is $365 \mathrm{~nm}$ )

\subsubsection{VITAMINS}

The main results of our own investigations of fluorescence properties of milk vitamins are presented in this section. In addition, the literature references are included to summarize fluorescence data for milk vitamins at room temperature.

Vitamin C (ascorbic acid) in aqueous solution demonstrates fluorescence excitation maximum at $369 \mathrm{~nm}$ with half-width of about 70 $\mathrm{nm}$ (Fig. 4.34) and fluorescence emission maximum at $460 \mathrm{~nm}$ with halfwidth of about $100 \mathrm{~nm}$ (Fig. 4.35).

Vitamin $B_{2}$ (riboflavine) is the most intense fluorophore of milk. Fluorescence excitation spectrum demonstrates the maxima at 370, 415

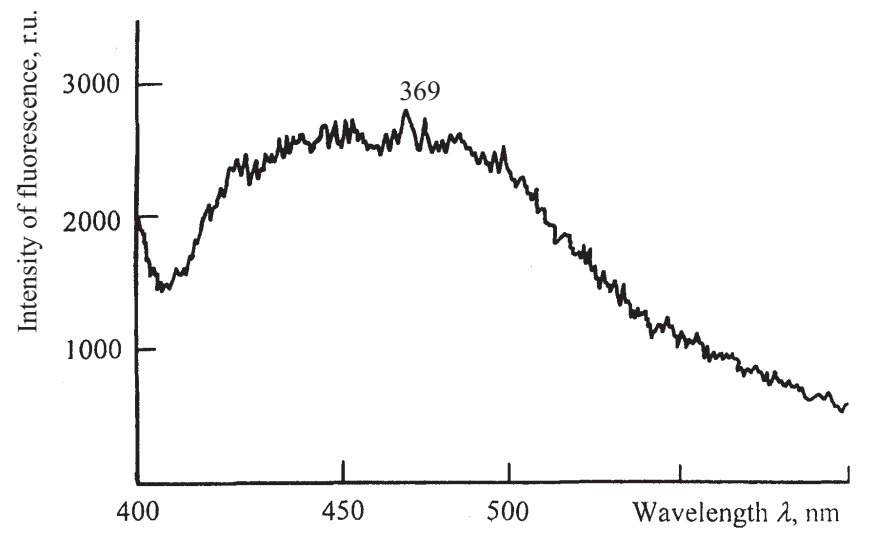

Fig. 4.34 Fluorescence excitation spectrum of vitamin $C$ (ascorbic acid) in aqueous solution 


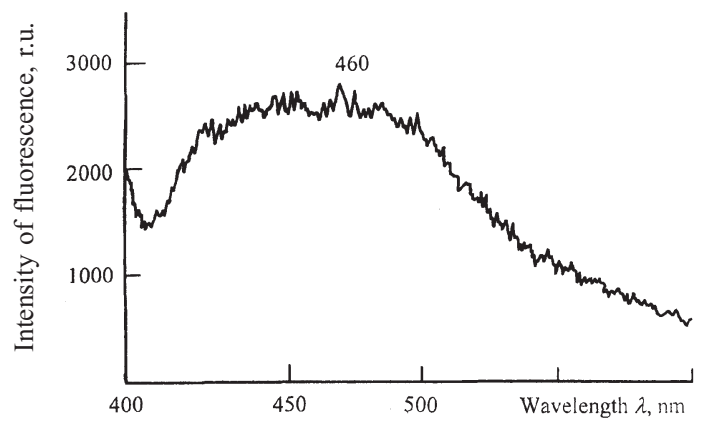

Fig. 4.35 Fluorescence emission spectrum of vitamin $C$ (ascorbic acid) in aqueous solution

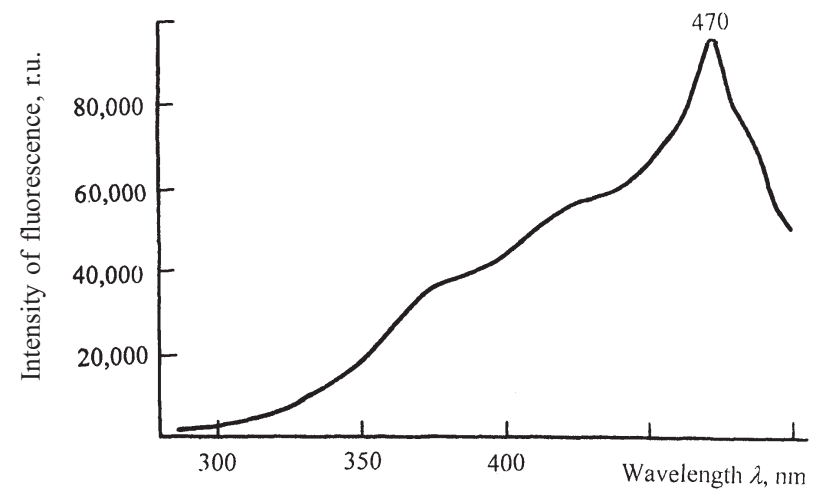

Fig. 4.36 Fluorescence excitation spectrum of vitamin $B_{2}$ (riboflavine) (emission wavelength is $520 \mathrm{~nm}$ )

and $470 \mathrm{~nm}$ (emission wavelength is $520 \mathrm{~nm}$ ) and 469 and $538 \mathrm{~nm}$ (emission wavelength is $563 \mathrm{~nm}$ ); these excitation spectra are presented in Figs. 4.36 and 4.37. It is possible to observe in intense and wide bands in fluorescence emission spectrum at $531 \mathrm{~nm}$ (excitation wavelength is $365 \mathrm{~nm}$ ) and at $521 \mathrm{~nm}$ (excitation wavelength is $399 \mathrm{~nm}$ ); these emission spectra are presented in Figs. 4.38 and 4.39.

Vitamin $B_{6}$ (pyridoxine) has two maxima at 367 and $400 \mathrm{~nm}$ in fluoresence excitation spectrum (emission wavelength is $471 \mathrm{~nm}$ ) (Fig. 4.40), which transform into one wide spectral band at $380-400 \mathrm{~nm}$ if emission wavelength is $500 \mathrm{~nm}$. Fluorescence emission spectrum demonstrates maximum at $470 \mathrm{~nm}$ (excitation wavelength is $367 \mathrm{~nm}$ ) and at $500 \mathrm{~nm}$ (excitation wavelength is $400 \mathrm{~nm}$ ); these emission spectra are presented in Figs. 4.41 and 4.42. 


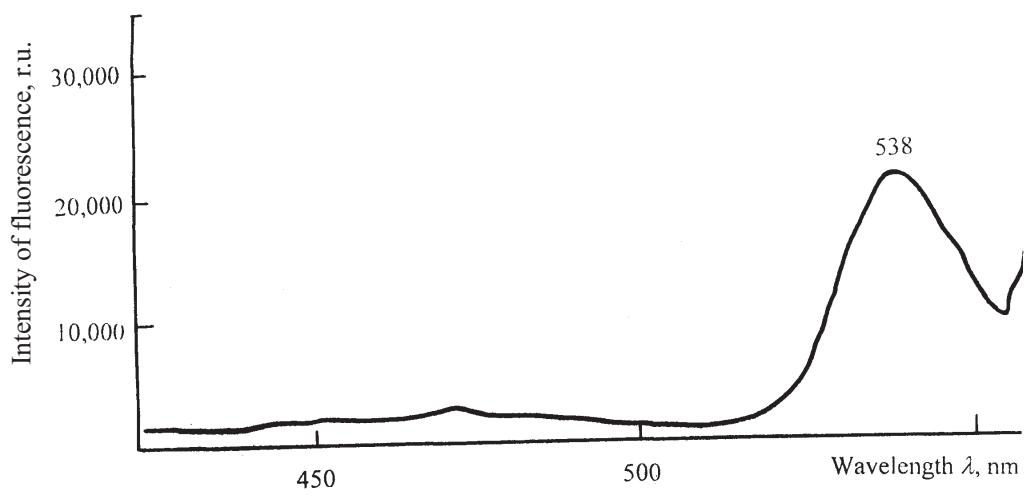

Fig. 4.37 Fluorescence excitation spectrum of vitamin $B_{2}$ (riboflavin) (emission wavelength is $563 \mathrm{~nm}$ )

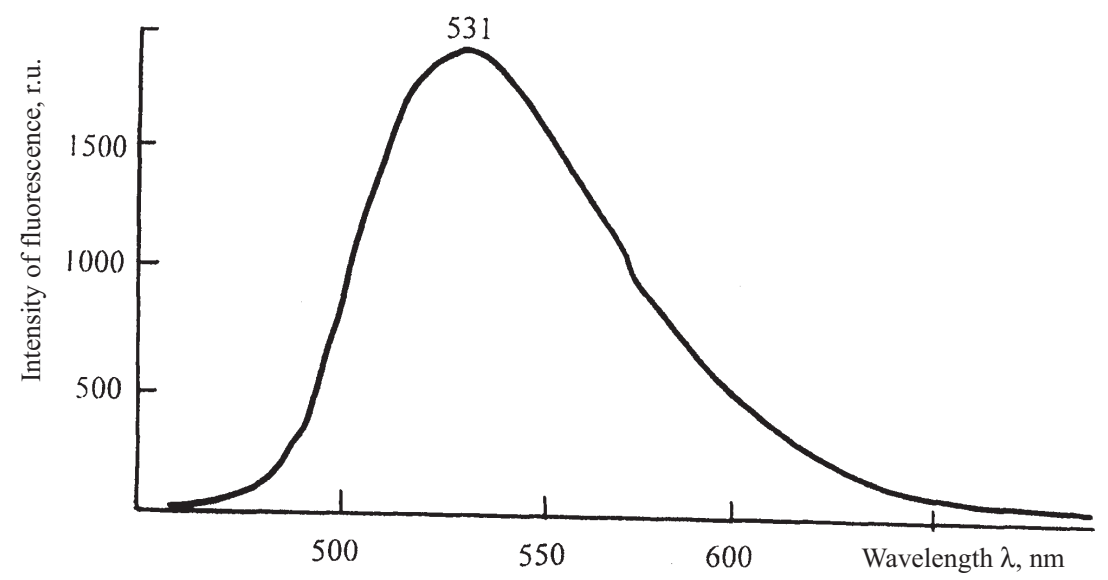

Fig. 4.38 Fluorescence emission spectrum of vitamin $B_{2}$ (riboflavin) (excitation wavelength is $365 \mathrm{~nm}$ )

Vitamin $B_{12}$ (cyanocobalamin) demonstrates fluorescence excitation maximum (Fig. 4.43) at $330 \mathrm{~nm}$ with half-width of about $25 \mathrm{~nm}$ (emission wavelength is $398 \mathrm{~nm}$ ) and fluorescence emission maximum (Fig. 4.44) at $400 \mathrm{~nm}$ (excitation wavelength is $345 \mathrm{~nm}$ ).

The literature data concerning fluorescence properties of milk vitamins are as follows:

Vitamin $E$ has maxima of fluorescence excitation at $295 \mathrm{~nm}$ and fluorescence emission at $340 \mathrm{~nm}$ [Udenfriend, 1985]. 


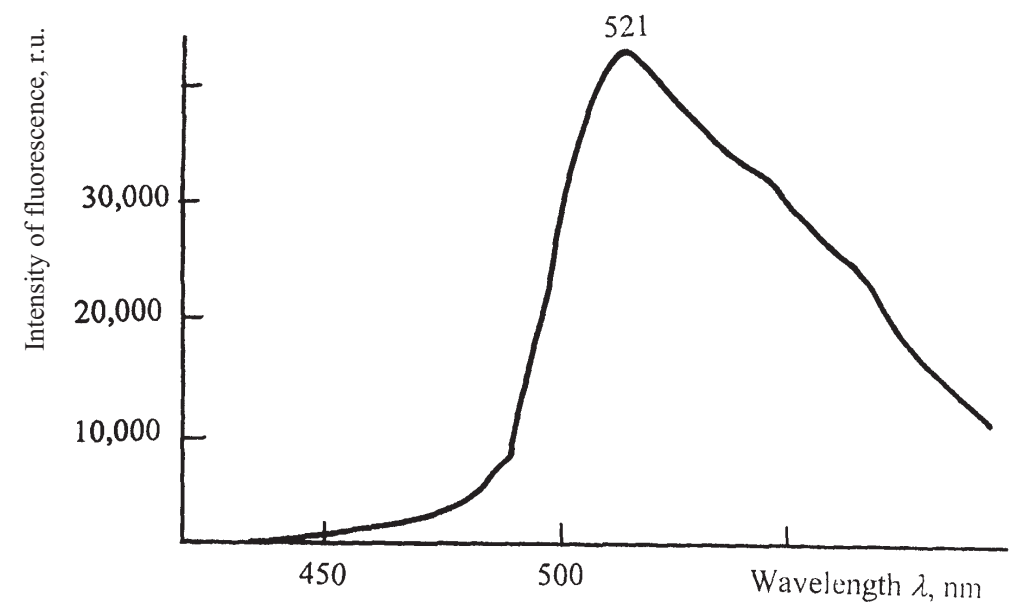

Fig. 4.39 Fluorescence emission spectrum of vitamin $B_{2}$ (riboflavin) (excitation wavelength is $399 \mathrm{~nm}$ )

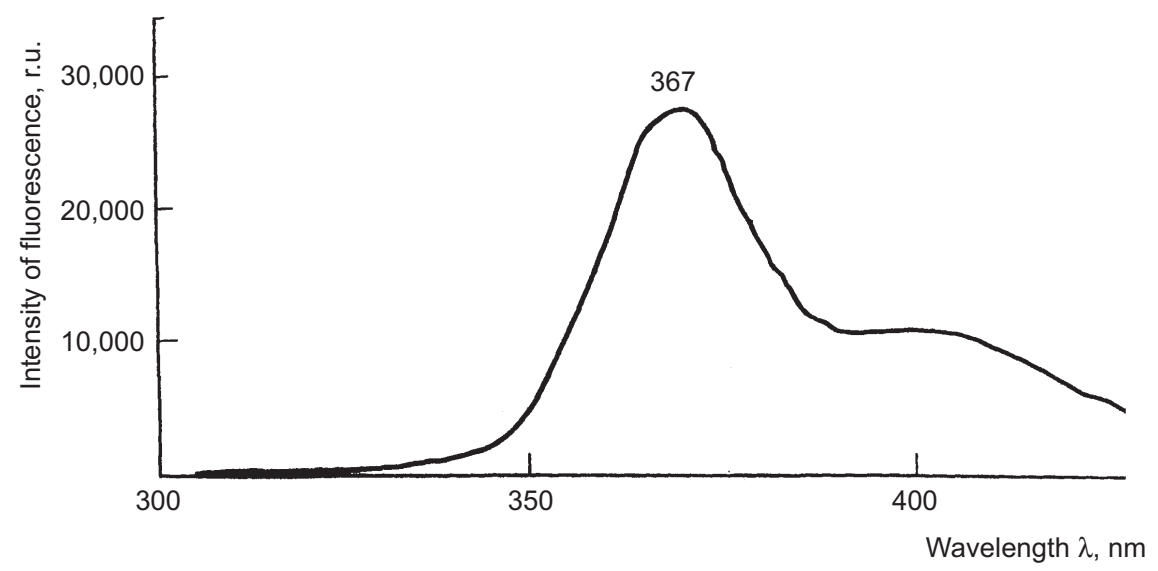

Fig. 4.40 Fluorescence excitation spectrum of vitamin $B_{6}$ (pyridoxine) (emission wavelength is $471 \mathrm{~nm}$ )

Vitamin $B_{1}$ (thiamine) in aqueous solutions does not demonstrate any fluorescence at room temperature [Krasnikov et al., 1987], but its oxidation product thiochrome shows maxima of fluorescence excitation at $365 \mathrm{~nm}$ and fluorescence emission at $450 \mathrm{~nm}$ [Vogi et al., 1955, cited by Udenfriend, 1985].

Vitamin $P P$ has amide of nicotine acid (nicotinamide) which demonstrates fluorescence excitation maximum at $385 \mathrm{~nm}$ and fluorescence emission maximum at $475 \mathrm{~nm}$ [Krasnikov et al., 1987]. 


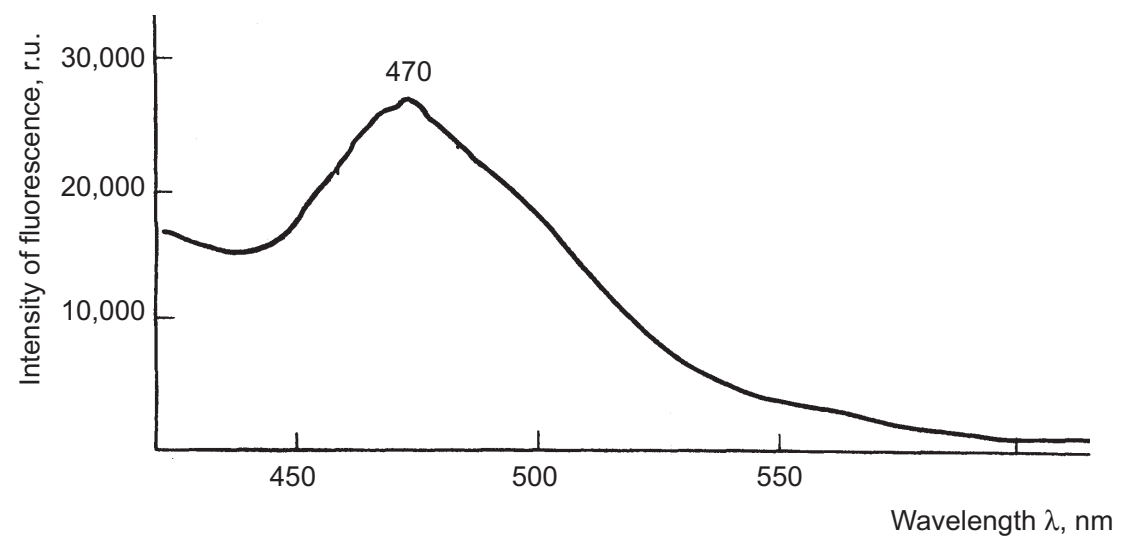

Fig. 4.41 Fluorescence emission spectrum of vitamin $B_{6}$ (pyridoxine) (excitation wavelength is $367 \mathrm{~nm}$ )

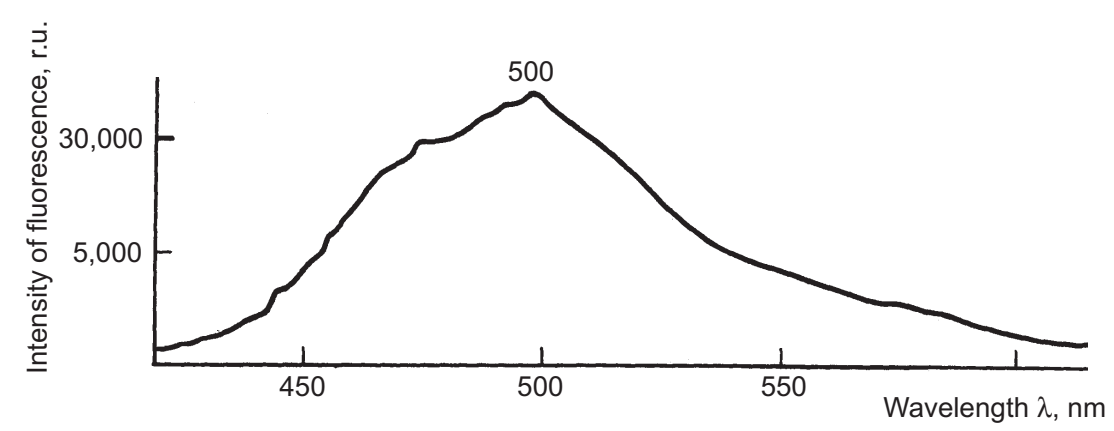

Fig. 4.42 Fluorescence emission spectrum of vitamin $B_{6}$ (pyridoxine) (excitation wavelength is $400 \mathrm{~nm}$ )

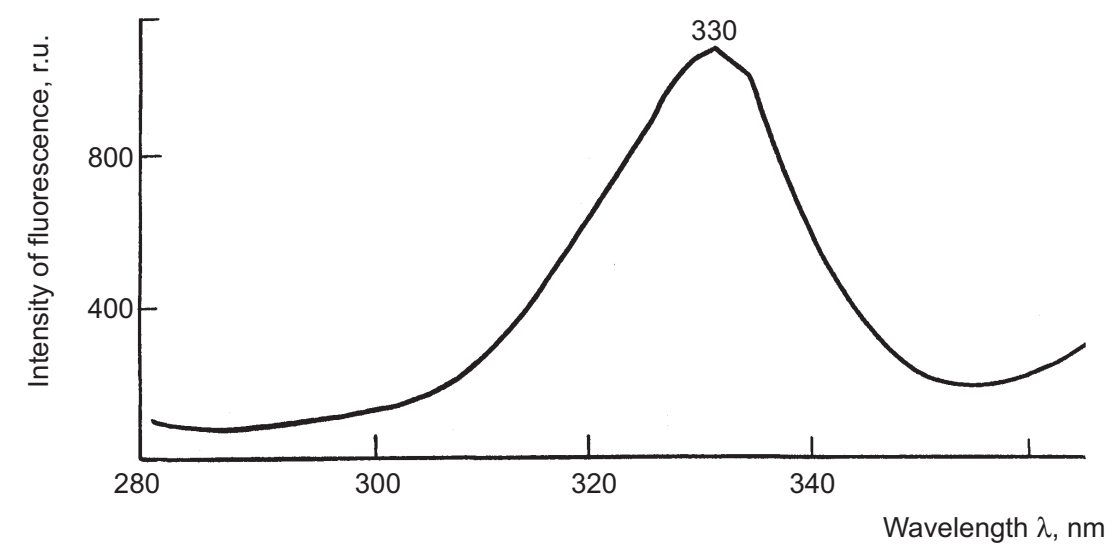

Fig. 4.43 Fluorescence excitation spectrum of vitamin $B_{12}$ (cyanocobalamin) (emission wavelength is $398 \mathrm{~nm}$ ) 


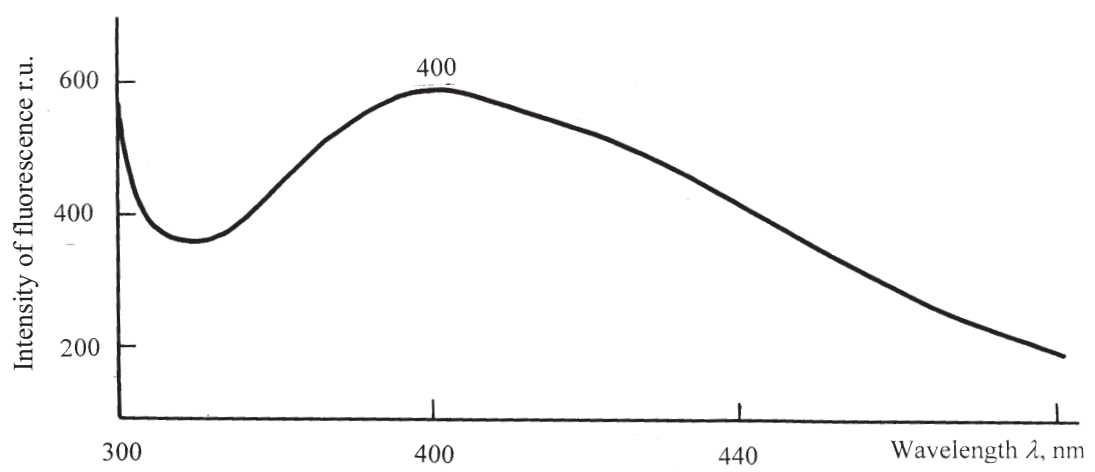

Fig. 4.44 Fluorescence emission spectrum of vitamin $B_{12}$ (cyanocobalamin) (excitation wavelength is $345 \mathrm{~nm}$ )

EVitamin $B_{3}$ (pantothenic acid) is unstable in free state; there are data Dut fluorescence properties of the salt of this acid only - calcium pantothenate; it has fluorescence excitation maximum at $385 \mathrm{~nm}$ (with small maxima at 229, 260, and $385 \mathrm{~nm}$ ) and fluorescence emission maximum at $460 \mathrm{~nm}$ [Krasnikov and Timoshkin, 1983; Timoshkin and Titkova, 1986].

Folic acid has fluorescence emission spectrum at $450 \mathrm{~nm}$ (excitation wavelength is $365 \mathrm{~nm}$ ) and at $370 \mathrm{~nm}$ (excitation wavelength is $290 \mathrm{~nm}$ ) [Daddan et al., 1957, cited by Udenfriend, 1985].

\subsection{IDENTIFICATION OF MILK FLUOROPHORES}

\subsubsection{Selection of Excitation Wavelength}

The first approach of the determination of milk components is related to the choice of the corresponding wavelength of excitation. For example, the excitation at $270-310 \mathrm{~nm}$ provokes the fluorescence emission of proteins and aromatic amino acids; excitation near 315-340 nm causes the fluorescence of vitamins $\left(B_{1}, B_{2}, B_{6}, B_{12}, A\right)$; excitation near 340-370 $\mathrm{nm}$ is responsible for the emission of fat acids and vitamins $\left(B_{1}, B_{2}, B_{3}, B_{6}\right.$, $C, D$, folic acid). The dependence of fluorescence emission of milk components on the excitation wavelength is presented in Table 4.10.

It is possible to vary the wavelength of excitation and to pick out a certain component (Fig. 4.45). For example, if the fluorescence of proteins at $340 \mathrm{~nm}$ is registered, one can be sure to avoid the influence of fat and riboflavin. There is a superposition of fluorescence emission bands of fat and riboflavin in the visible $(400-700 \mathrm{~nm})$ part of the spectrum; the application of interference filters makes it possible to select fluorescence of each milk component (Fig. 4.46). 


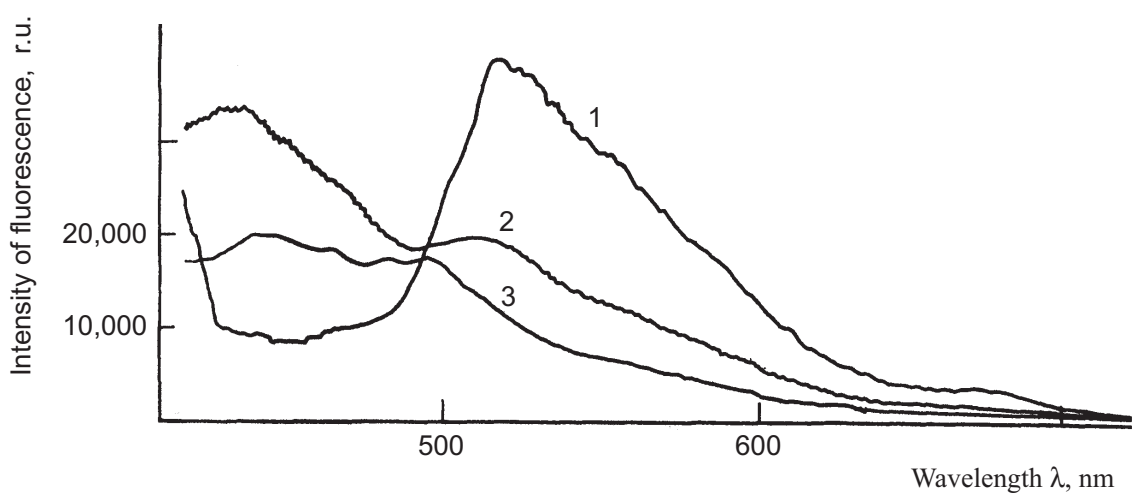

Fig. 4.45 Variation of the wavelength of excitation to pick out a certain component of milk: 1 - riboflavin; 2 - fatty acids; 3 - milk. Excitation wavelengths are: $400 \mathrm{~nm}-$ riboflavin; $360 \mathrm{~nm}$ - fatty acids; $340 \mathrm{~nm}$ - milk

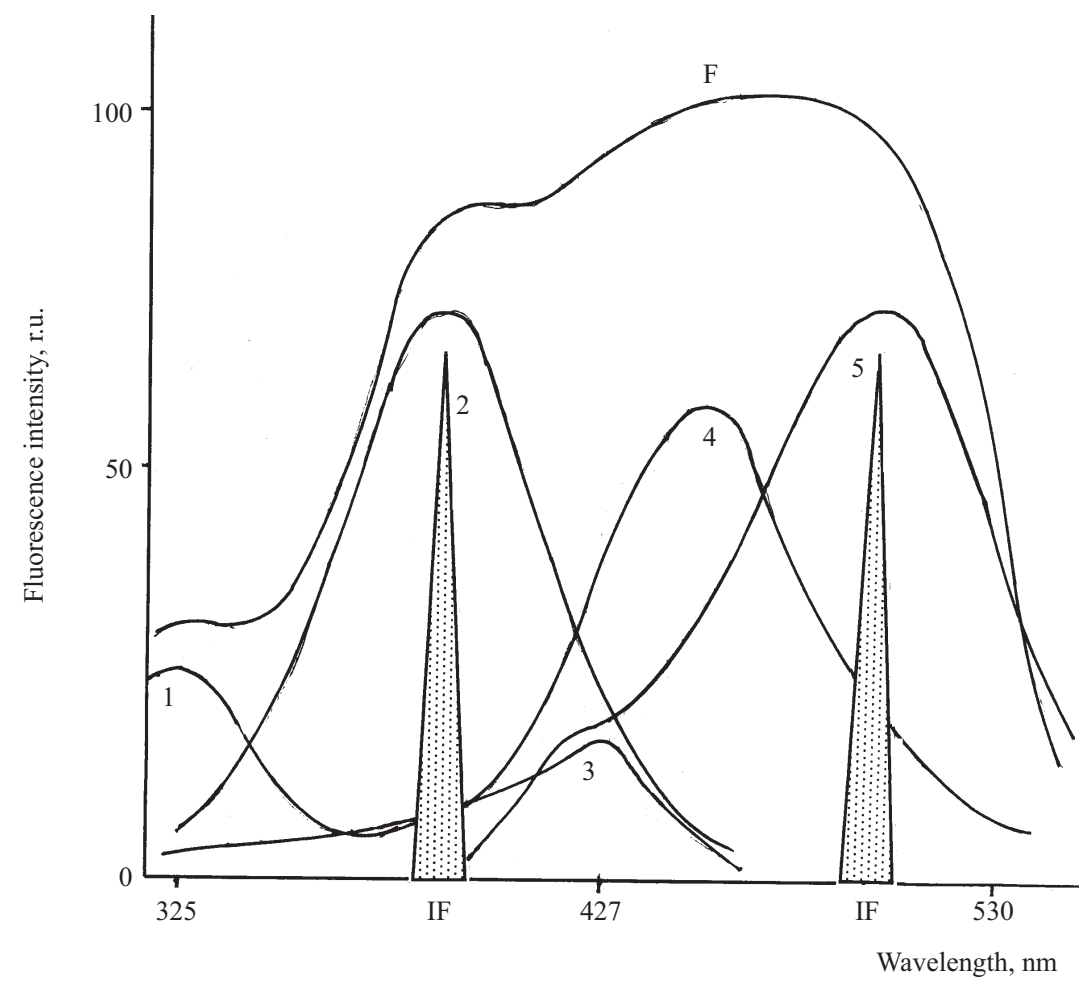

Fig. 4.46 The application of interference filters to select fluorescence of each milk component: $\mathrm{F}$ - spectral band of milk, 1-5 - spectral bands of milk components; IF transmission bands of interference filters 


\subsubsection{Decomposition of Fluorescence Spectra}

The second approach provides the determination of the fluorescence curve for a number of components; each of them is characterized by the relative intensity, wavelength, half-width and shape. The cooling of milk (up to $77 \mathrm{~K}$ ) induces the appearance of its fine structure and facilitates the quantitative and qualitative analysis of milk components.

We have elaborated the spectra of fluorescence of milk with a universal multiparametrical and multifunctional package of programmes (UPOS) [Kucherov, 1984]. Therefore, the fluorescence emission spectrum of cooled milk (excitation wavelength is $313 \mathrm{~nm}$ ) can be decomposed as five spectral bands (Table 4.11).

Table 4.11 Decomposition of fluorescence emission spectrum of cooled milk (excitation wavelength is $313 \mathrm{~nm}$ )

\begin{tabular}{c|c|c|c|c|c|r}
\hline $\begin{array}{c}\text { Band } \\
\text { Number }\end{array}$ & $\begin{array}{c}\text { Intensity of } \\
\text { Band (Relative } \\
\text { Units) }\end{array}$ & $\begin{array}{c}\text { Wavelength } \\
(\mathrm{nm})\end{array}$ & $\begin{array}{c}\text { Half-width } \\
(\mathrm{nm})\end{array}$ & \multicolumn{2}{|c|}{ Form-Factor } & \multicolumn{2}{|c}{$\begin{array}{c}\text { Surface of the } \\
\text { Band } \\
\text { (Relative Units) }\end{array}$} \\
\hline & & & & $F_{1}$ & $F_{2}$ & \\
\hline 1 & 89.24 & 333.0 & 31.58 & 0.41 & 0.60 & 548.03 \\
2 & 257.16 & 381.0 & 51.83 & 0.90 & 0.72 & $2,299.71$ \\
3 & 66.02 & 409.0 & 27.63 & 0.60 & 0.93 & 323.59 \\
4 & 213.79 & 430.0 & 52.35 & 0.69 & 0.20 & $2,368.57$ \\
5 & 260.55 & 463.0 & 59.47 & 0.06 & 1.00 & $3,531.39$ \\
\hline
\end{tabular}

It is possible to identify the following milk components such as tryptophan $(\mathrm{N} 1,333 \mathrm{~nm})$, vitamin $B_{6}(\mathrm{~N} 2,381 \mathrm{~nm})$, fat acids (N3, 409 $\mathrm{nm})$, vitamin $B_{6}(\mathrm{~N} 4,430 \mathrm{~nm})$, and vitamin $C(\mathrm{~N} 5,463 \mathrm{~nm})$. The results of decomposition are given in Fig. 4.47.

The fluorescence emission spectrum of cooled milk (excitation wavelength is $365 \mathrm{~nm}$ ) can be decomposed in to two components (Table 4.12); the first components (447.52 nm) can be identified as fluorescence emission of vitamins $D, B_{1}$, and $A$; the second one $(496.34 \mathrm{~nm})$ can be associated with fluorescence emission of riboflavin.

In such a way, the procedure of decomposition of fluorescence spectra of milk is one of the perspective methods of identification of milk components. It is possible to confront parameters of spectral bands with the factors which affect the biological value of milk. 

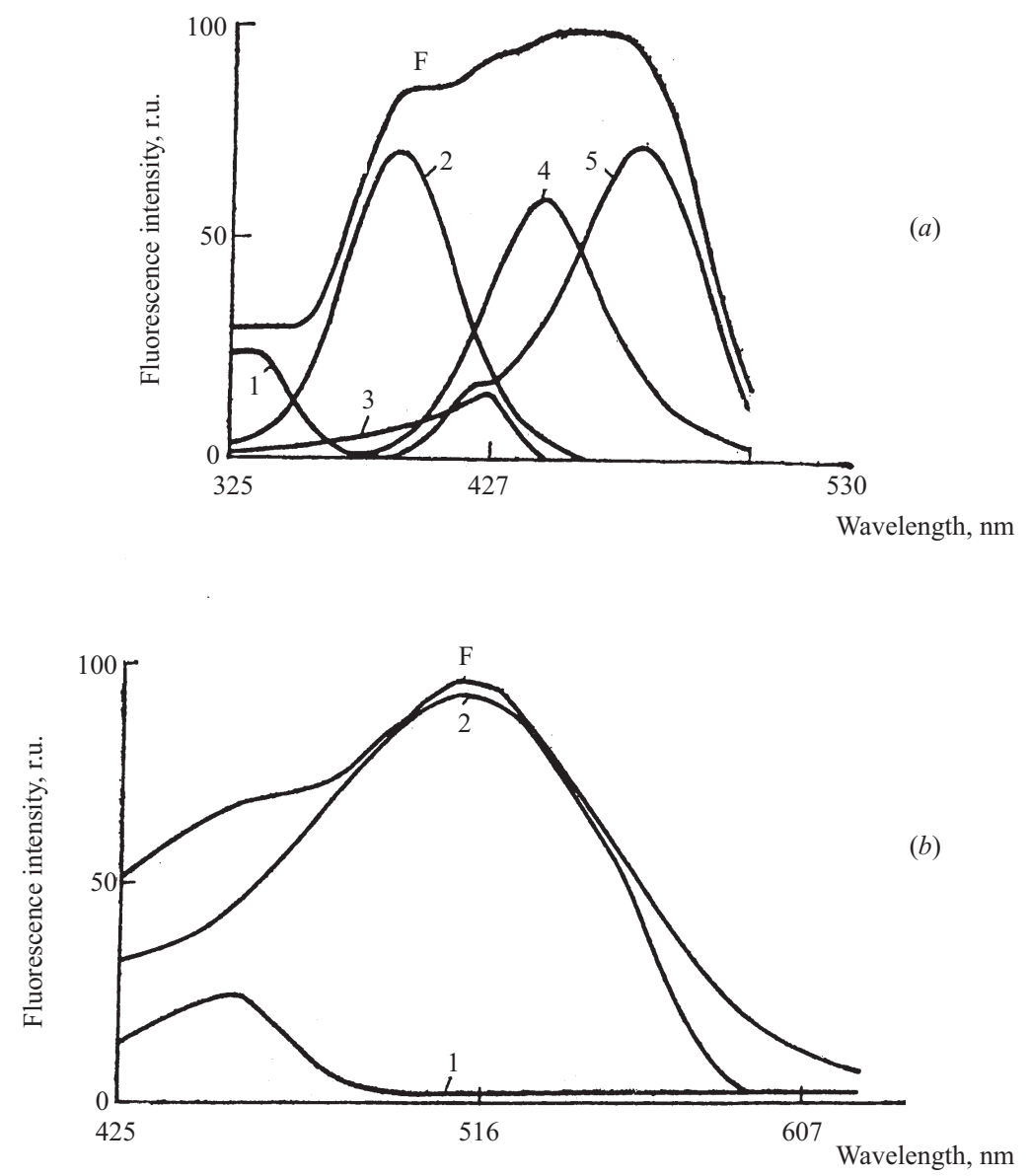

Fig. 4.47 The results of decomposition of fluorescence spectra of milk at the temperature of liquid nitrogen: $(a)$ excitation wavelength is $313 \mathrm{~nm}$; $\mathrm{F}-$ spectral band of milk, 1-5 spectral bands of milk components; (b) excitation wavelength is $365 \mathrm{~nm}$; F - spectral band of milk, 1-2 - spectral bands of milk components

Table 4.12 Decomposition of fluorescence emission spectrum of cooled milk (excitation wavelength is $365 \mathrm{~nm}$ )

\begin{tabular}{c|c|c|c|c|c|c}
\hline $\begin{array}{c}\text { Band } \\
\text { Number }\end{array}$ & $\begin{array}{c}\text { Intensity of } \\
\text { Band (Relative } \\
\text { Units) }\end{array}$ & $\begin{array}{c}\text { Wavelength } \\
(\mathrm{nm})\end{array}$ & $\begin{array}{c}\text { Half-width } \\
(\mathrm{nm})\end{array}$ & \multicolumn{2}{|l|}{ Form - Factor } & $\begin{array}{c}\text { Surface of the } \\
\text { Band } \\
\text { (Relative Units) }\end{array}$ \\
\cline { 5 - 6 } & 57.68 & 447.52 & 32.89 & 0.91 & 1.0 & 188.12 \\
2 & 226.39 & 496.34 & 90.41 & 0.06 & 1.0 & $2,685.69$ \\
\hline
\end{tabular}




\subsection{FLUOROMETERS FOR MILK ANALYSIS}

\subsubsection{Fluorometer for Determination of Protein and Fat Content in Milk}

A design of fluorometer for determination of protein and fat content in milk is presented in Fig. 4.48. Mercury lamp 1 of high pressure DRK-120 was used as the source of fluorescence excitation. The radiation of this lamp was collimated by concave mirror 2, lens 2 and 4, and passed through interference filter 3 (280 or $325 \mathrm{~nm}$ ), cuvette 6 with the sample (the windows of this cuvette were performed with quartz), and lens 7. The fluorescence emission was registered at the angle $30^{\circ}$ to the excitation radiation in order to avoid the effect of reflected and scattered radiation. The readout system consisted of the cut-off filters 8 (CZC-22 or CZC-23), and photomultiplier 9 FEU-79, amplifier 10 and recorder 11.

The dependence of the intensity of fluorescence on the level of dilution of milk is presented in Fig. 4.49 for protein and in Fig. 4.50 for fat. This dependence demonstrates linear character in the range of concentrations $4-5 \mathrm{~g} / \mathrm{L}$.

\subsubsection{Laser-induced Fluorometry of Milk}

The $\mathrm{N}_{2}$-laser fluorometer ( $337 \mathrm{~nm}$ ) was used for analysis of fluorescence emission spectra of dairy products: milk, baked milk, whey and sour

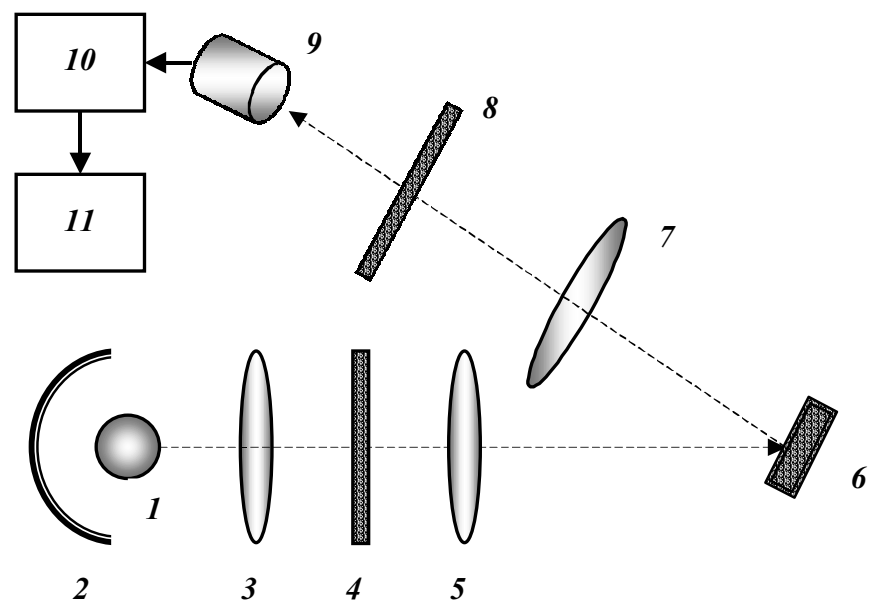

Fig. 4.48 A design of fluorometer for determination of protein and fat content in milk. 1 mercury lamp of high pressure DRK-120 ; 2 - concave mirror; 3, 5-lens; 4 -interference filter (280 or $325 \mathrm{~nm}$ ); 6 - cuvette with the sample; 7 - lens; 8 - cut-off filters (CZC-22 or CZC-23); 9 - photomultiplier FEU-79; 10 - amplifier; 11 - recorder 


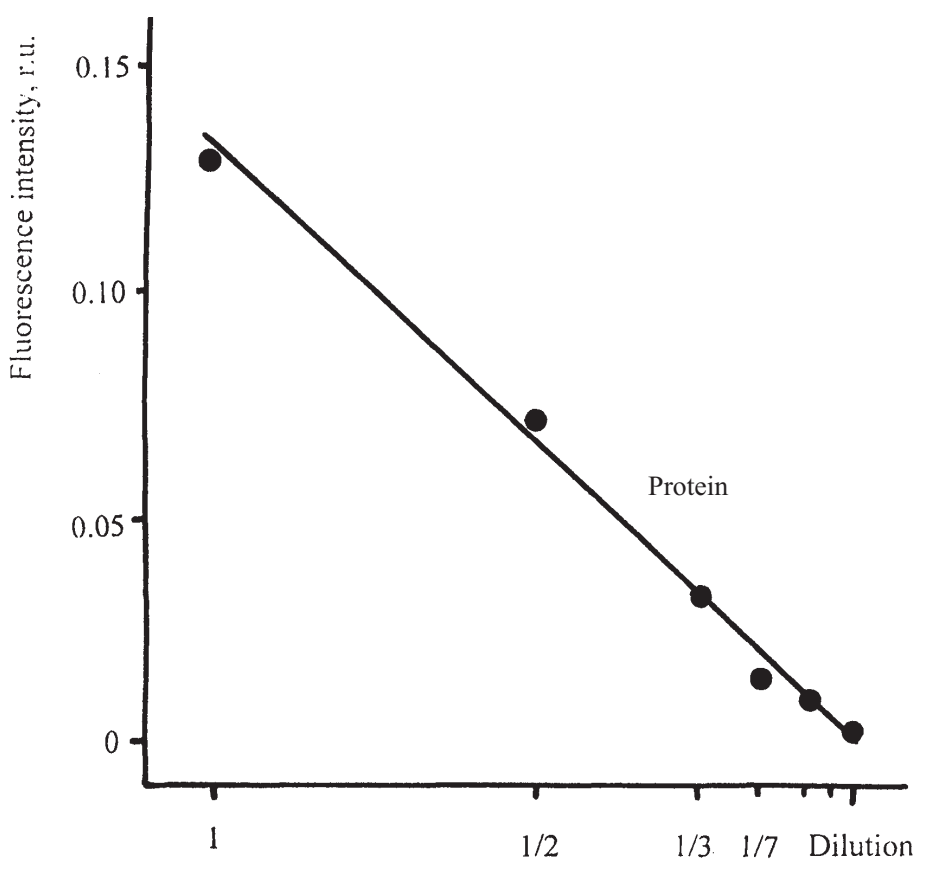

Fig. 4.49 Dependence of the intensity of fluorescence of protein on the level of dilution of milk in the range of concentrations $4-5 \mathrm{~g} / \mathrm{L}$

cream. The analysis of laser-induced spectra of milk made it possible to estimate concentrations of fat in milk samples [Posudin and Kostenko, 1994]. High level of correlation between the results which were obtained during chemical and laser-fluorometric analysis testifies in favour of laser fluorometry as method of quantitative evaluation of dairy products.

The schematic representation of laser fluorometer used [Posudin and Kostenko, 1994] for the determination of fat is given in Fig. 4.51. The radiation of nitrogen laser 1 was split by a semitransparent glass plate 2 into two beams - the first one was directed to the cuvette 3 with milk, and the second one - to fluorescence standard (solution of fluorescein) 4 . The fluorescence emission was detected in both cases with two photomultipliers 6 and 7. The first photomultiplier was equipped with the interference filter $5(400 \mathrm{~nm})$ for selection of fluorescence emission of fatty acids. The electrical signals of both photomultipliers were directed to the differential amplifier 8 and to readout system 9 . Such a differential system can estimate the ratio of fluorescence intensities of the sample and fluorescent standard; the possible fluctuations of the laser radiation or the power supply do not affect the results of fat determination. 
68 Practical Spectroscopy in Agriculture and Food Science

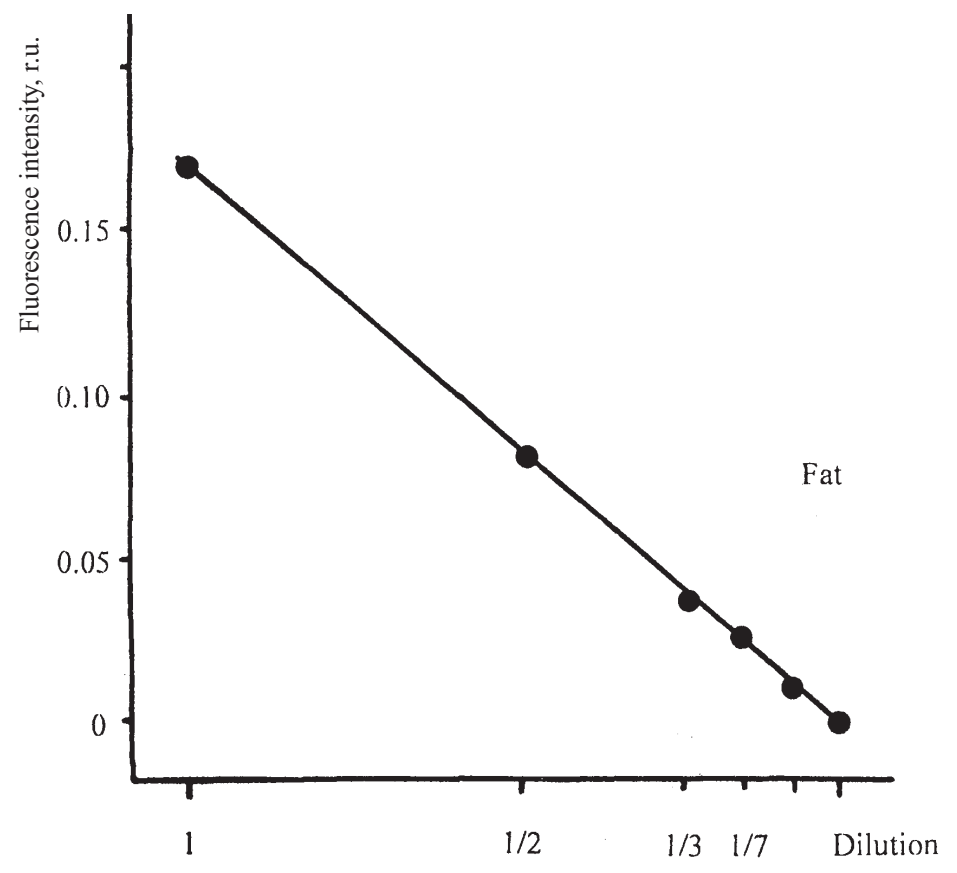

Fig. 4.50 Dependence of the intensity of fluorescence of fat on the level of dilution of milk in the range of concentrations $4-5 \mathrm{~g} / \mathrm{L}$

It is necessary to mention that the dependence of fluorescence intensity $I_{F l}$ on the concentration $C$ of the sample is determined as

$$
I_{F l}=I_{0}\left(1-10^{-\varepsilon C l}\right) \phi
$$

where $I_{0}$ is the intensity of the incident radiation, $\varepsilon$ - molar absorption, $l-$ optical pathlength, $\phi$ - quantum yield of fluorescence.

If $\varepsilon C \lambda<<0.05$, this equation can be transformed as follows:

$$
I_{F l}=I_{0}(2.3 \varepsilon C l) \phi
$$

Dependence of the intensity of fluorescence of milk on the level of dilution is presented in Fig. 4.52, and on the temperature in Fig. 4.53.

Thus, the fluorescence intensity depends linearly on the concentration of the sample for diluted solutions only; this method can be used in laboratory measurements. 


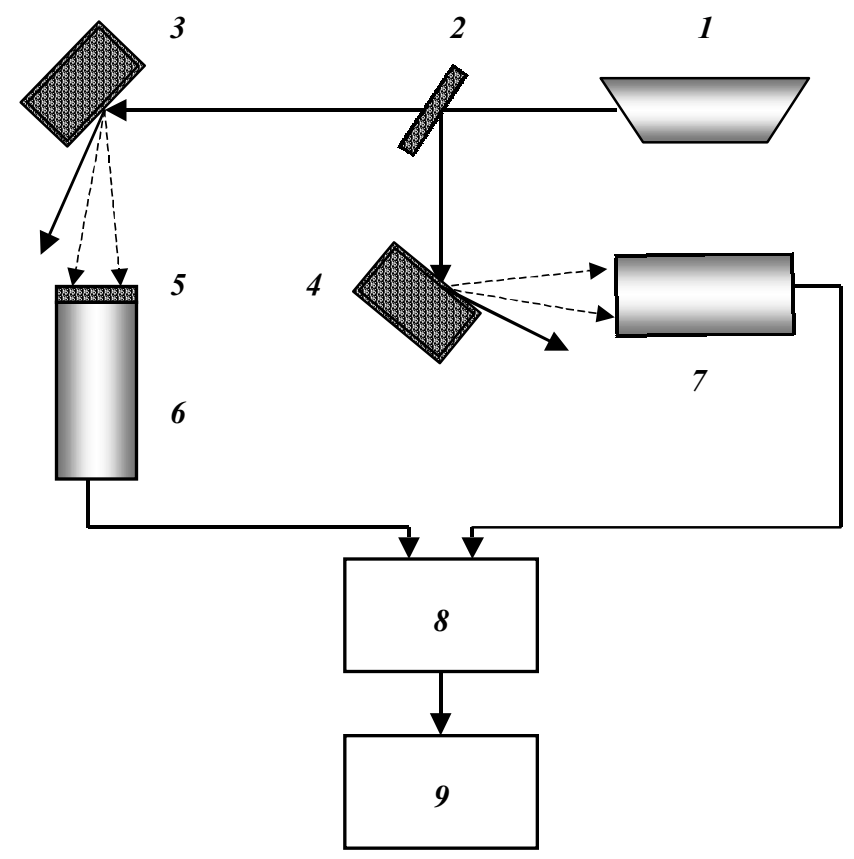

Fig. 4.51 The schematic representation of laser fluorometer used for the determination of fat [Posudin and Kostenko, 1994]. 1 - nitrogen laser; 2 - semitransparent glass plate; 3 cuvette with milk; 4 - fluorescence standard (solution of fluorescein); 5 - interference filter (400 nm); 6, 7 - photomultipliers; 8 - differential amplifier; 9 - readout system

\subsection{LASER LIGHT SCATTERING BY MILK PARTICLES}

\subsubsection{Theory of Light Scattering}

Theoretically the process of light scattering is characterized by the socalled coefficient of scattering $Q$ [Hulst, 1982; Walstra, 1964]. This coefficient depends on the wavelength of light $\lambda$, refractive indices of particles $n_{1}$ and medium $n_{2}$, and the size $d$ of particles. The effect of these factors is determined with such parameters as relative refraction index $m=n_{1} / n_{2}$, and phase shift $\rho=2 x(m-1)$, where $x=\pi d / \lambda$. There are different types of scattering depending on the values of all these parameters. The complete theory of light scattering is given in [Hulst, 1982]; several examples of the relation of coefficient of scattering with above-mentioned parameters are listed below.

1. Rayleigh Scattering. This type of scattering is demonstrated by the spherical particles which have relative refractive index equal to about 1 and dimensions less than the wavelength: 
70 Practical Spectroscopy in Agriculture and Food Science

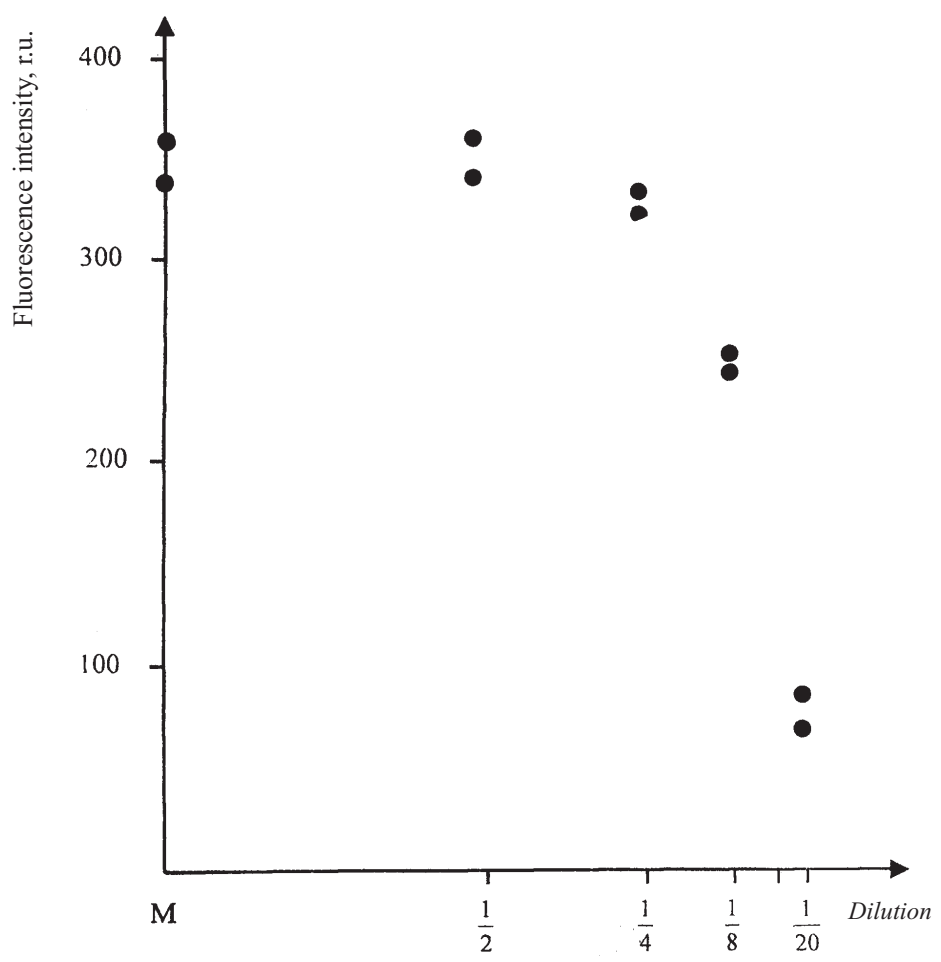

Fig. 4.52 Dependence of the intensity of laser-induced fluorescence of milk on the level of dilution of milk

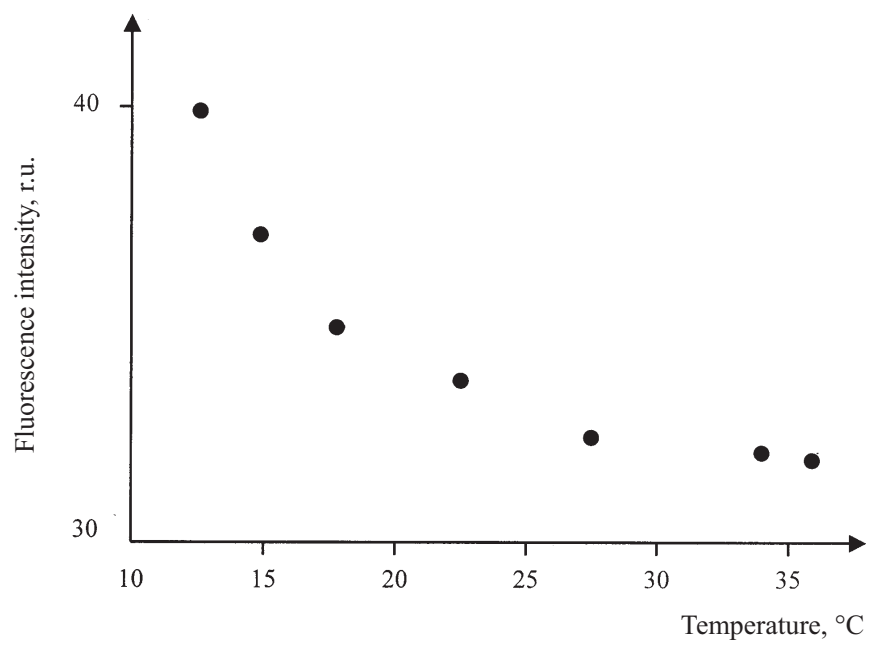

Fig. 4.53 Dependence of the intensity of laser-induced fluorescence of milk on the temperature of the sample 


$$
\begin{gathered}
m \rightarrow 1 \\
Q_{R}=\frac{32}{27} x^{4}(m-1)^{2}
\end{gathered}
$$

The following expression was proposed for very small $(m x<<1)$ particles [Walstra, 1965]:

$$
Q_{R}=\frac{8}{3} x^{4}\left(\frac{m^{2}-1}{m^{2}+2}\right)^{2}
$$

2. Rayleigh-Gans Scattering. There are two possible situations for the particles which have relative refractive index equal to about 1 and very small phase shift $2 x(m-1)<1$ :

a. Rayleigh Scattering $x<1 ; Q_{R}=\frac{32}{27} x^{4}(m-1)^{2}$

b. Rayleigh-Gans Scattering $x>>1 ; Q_{R G}=2(m-1)^{2}$

3. Anomalous Diffraction. This type of scattering is demonstrated by the spherical particles which have relative refractive index equal to about 1 and dimensions which exceed the wavelength:

$$
\begin{gathered}
m \rightarrow 1 ; x>>1 ; \rho>> \\
Q_{N}=2-\frac{16 m^{2} \sin \rho}{(m+1)^{2} \rho}+4 \frac{1-m \cos \rho}{\rho^{2}}+7.54 \frac{z-m}{z+m} x^{-0.772}
\end{gathered}
$$

where $z=\left[\left(m^{2}-1\right)(6 x / \pi)^{2 / 3}+1\right]^{1 / 2}$.

If the dimensions of particles are small or moderate, the coefficient of scattering can be written as:

$$
Q_{S}=(1.26 m-0.44) \rho-2.558(m-1)^{1.273}-0.843
$$

\subsubsection{Application of Theory of Light Scattering to Milk Particles}

Milk is a complex mixture of fat globules $(0.1-10 \mu \mathrm{m})$, micelles of casein (0.1-0.2 $\mu \mathrm{m})$, and particles of serum proteins $(0.01-0.02 \mu \mathrm{m})$, and the turbidity of milk depends on the dimensions and concentration of these components. The measurement of the turbidity or optical density of milk can give information about the content of certain components. The method of light scattering seems to be one of the perspective approaches for the investigation of the size distribution of milk particles. 
Table 4.13 Dependence of coefficient of scattering on the spectral region and parameters $x, \rho$, and $d$

\begin{tabular}{l|c|c|c|c|c|c|c|c|c}
\hline Milk Particles & \multicolumn{2}{|c|}{$\begin{array}{c}\text { Ultraviolet Region } \\
(\lambda=0.2 \mu \mathrm{m})\end{array}$} & \multicolumn{3}{|c|}{$\begin{array}{c}\text { Visible Region } \\
(\lambda=0.6 \mu \mathrm{m})\end{array}$} & \multicolumn{3}{|c|}{$\begin{array}{c}\text { Infrared Region } \\
(\lambda=1.2 \mu \mathrm{m})\end{array}$} \\
\hline $\begin{array}{l}\text { Fat globules } \\
d>>\lambda\end{array}$ & & & & & & & & & \\
$d_{\min }=1.2 \mu \mathrm{m}$ & 15.71 & 3.14 & $Q_{N}$ & 5.0 & 1.0 & $Q_{N}$ & 2.62 & $\begin{array}{c}0.52 \\
1.6<\rho<2.5 \\
5.2 \\
\rho>>2.5\end{array}$ & $Q_{S}$ \\
$d_{\max }=10 \mu \mathrm{m}$ & 157.1 & 31.4 & $Q_{N}$ & 49.9 & 10.0 & $Q_{N}$ & 26.12 & $Q_{N}$ \\
\hline $\begin{array}{l}\text { Casein micelles } \\
d<\lambda\end{array}$ & & & & & & & & & \\
$d \sim 0.15 \mu \mathrm{m}$ & 2.36 & 0.47 & $Q_{R G}$ & 0.75 & 0.15 & $Q_{R}$ & 0.39 & 0.08 & $Q_{R}$ \\
\hline $\begin{array}{l}\text { Casein micelles } \\
d<\lambda\end{array}$ & & & & & & & & & \\
$d \sim 0.015 \mu \mathrm{m}$ & 0.24 & 0.05 & $Q_{R}$ & 0.075 & 0.015 & $Q_{R}$ & 0.04 & 0.01 & $Q_{R}$ \\
\hline
\end{tabular}

The dependence of coefficient of scattering on the spectral region and parameters such as $x, \rho$, and $d$ is given in Table 4.13.

Dependence of the coefficient of scattering on the wavelength of light is presented in Figs. 4.54 and 4.55 for milk globules, and in Figs. 4.56 and 4.57 for casein micelles and serum protein particles. Thus, the analysis of the dependence of light scattering on the wavelength and angle of

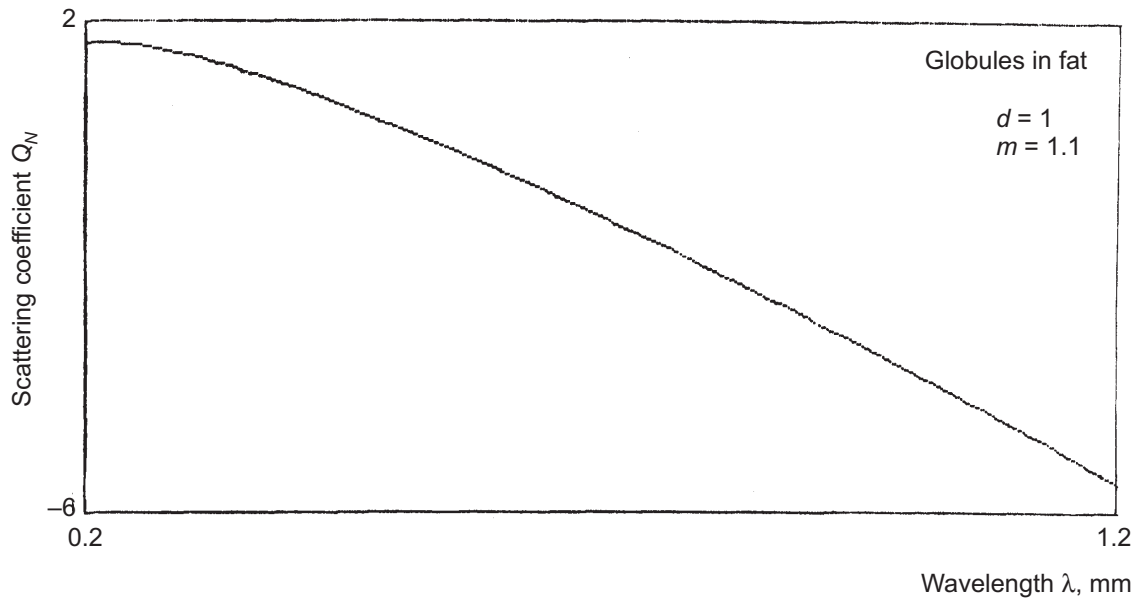

Fig. 4.54 Dependence of the coefficient of scattering on the wavelength of light for milk globules (diameter $1 \mu \mathrm{m}$ ) 


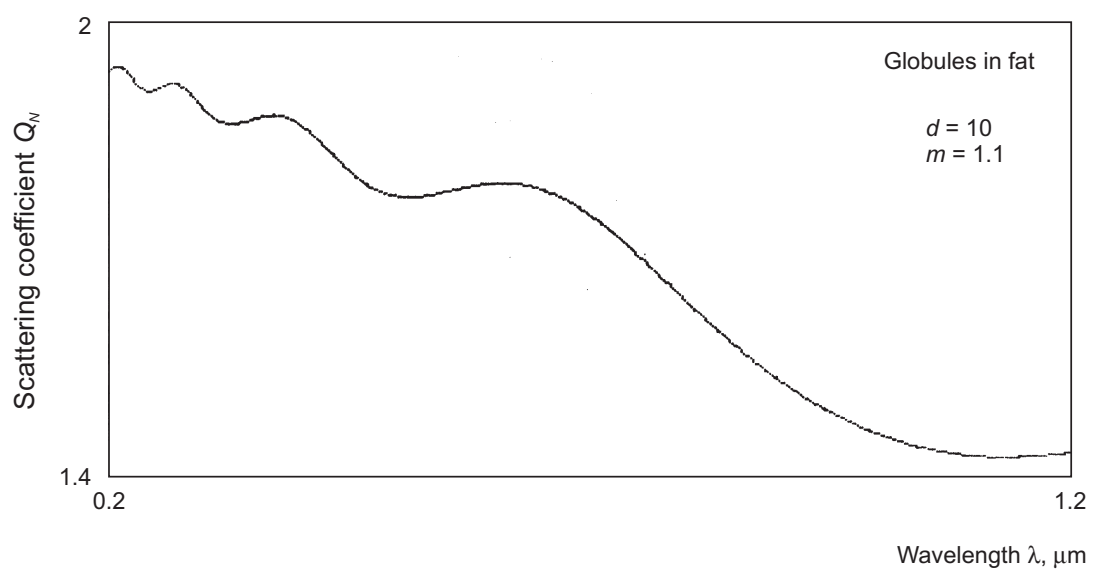

Fig. 4.55 Dependence of the coefficient of scattering on the wavelength of light for milk globules (diameter $10 \mu \mathrm{m}$ )

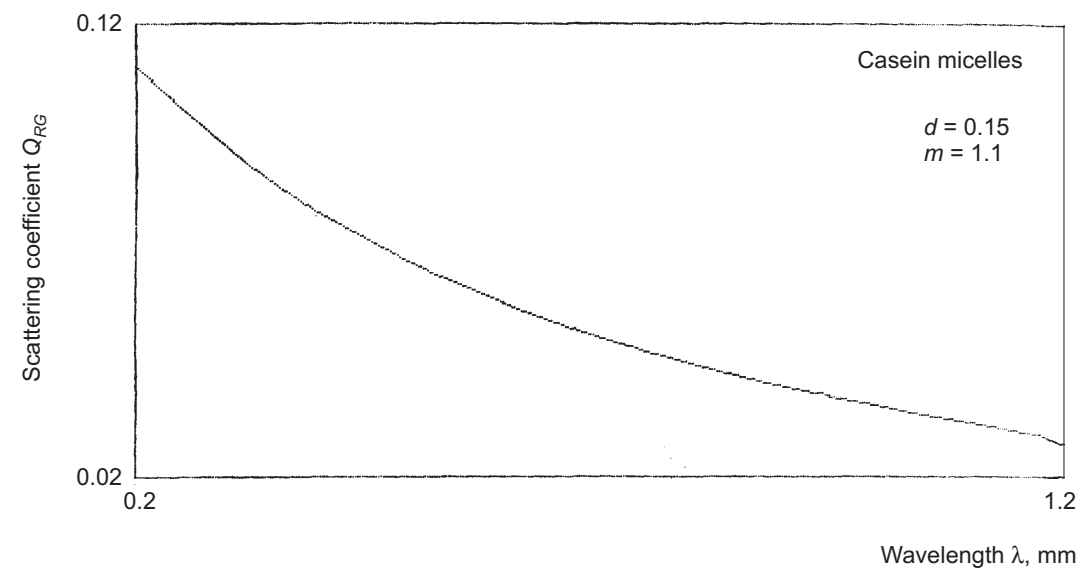

Fig. 4.56 Dependence of the coefficient of scattering on the wavelength of light for casein micelles

observation can make it possible to estimate quantitatively the dimension of milk particles.

It is clear that the transfer from visible to infrared part of the spectrum induces the decreasing of coefficient $Q_{N} 1.35$ times for fat globules of diameter $10 \mu \mathrm{m}$ and 4.41 times for fat globules of diameter 1 $\mu \mathrm{m}$. That is why it is expedient to pass to the infrared region in order to determine the fat content in milk and to avoid the effect of small particles. 
74 Practical Spectroscopy in Agriculture and Food Science

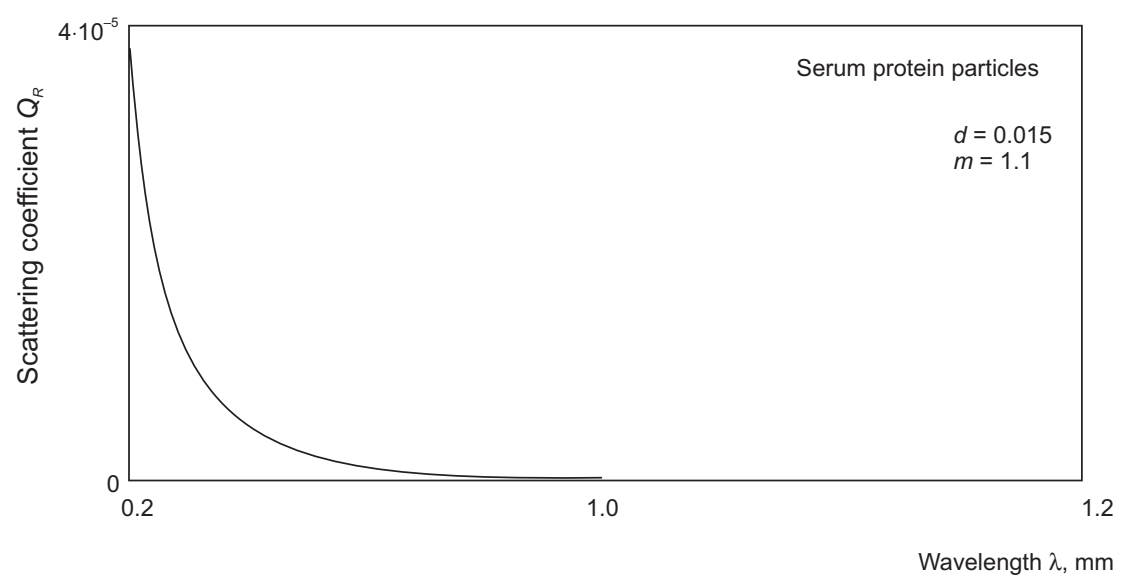

Fig. 4.57 Dependence of the coefficient of scattering on the wavelength of light for serum protein particles

\subsection{INSTRUMENTATION}

The experimental investigation of light scattering by milk particles was done with the photon correlation spectrometer System 4700c. The schematic representation of this spectrometer is presented in Fig. 4.58. The focused radiation of laser 1 is directed to the sample 2 of milk that is placed on the goniometer table 3. The scattered radiation is directed to the readout system which consists of photomultiplier 4, correlator 5, computer 6 , and printer 7 . A sample holder is supplied with a system of temperature control 8, peristaltic pump 9, and electric motor 10. A signal from the output of photomultiplier gives the information about the dependence of light scattering on the angle of observation. Milk, aqueous solution of lactose, dry milk, and milk mixtures were used as the samples.

\subsection{RESULTS OF LIGHT SCATTERING BY MILK PARTICLES}

The typical histograms of size distribution of these samples are given in Figs. 4.59-4.63. It is clear that the maximum of size distribution of milk particles is shifted with increasing concentration of particles from 100$500 \mathrm{~nm}\left(C \leq 10^{-3} \%\right)$ to $500-2,000 \mathrm{~nm}(C=7.4 \%)$. The character of size distribution of milk particles depends strongly on the concentration (Fig. 4.59-4.62) and the heating of the sample (Figs. 4.62-4.63). 


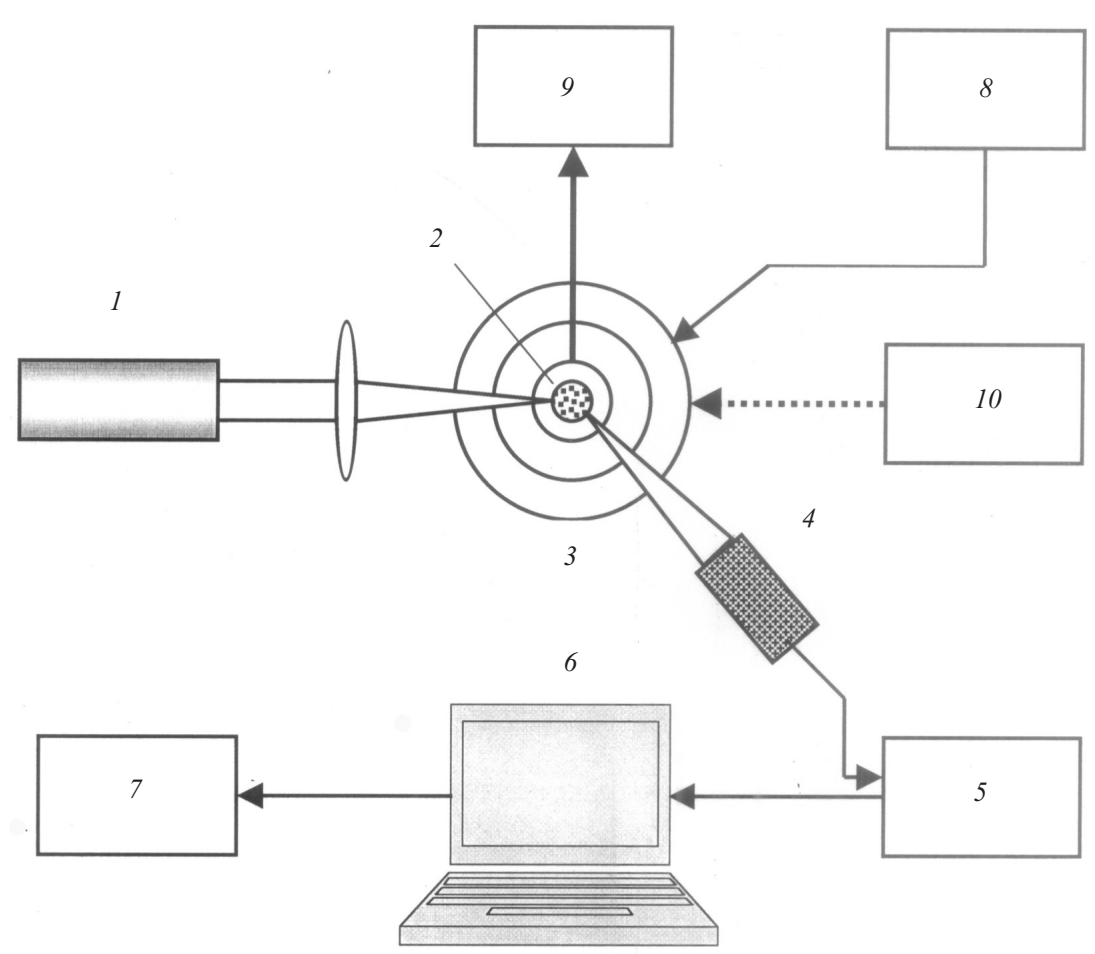

Fig. 4.58 Schematic representation of photon correlation spectrometer System 4700c. 1 - laser; 2 - sample; 3 - goniometer table; 4 - photomultiplier; 5 - correlator; 6 computer; 7 - printer; 8 - system of temperature control; 9 - peristaltic pump; 10 - electric motor

Fig. 4.64 demonstrates the dependence of the intensity of scattered light on the angle of observation.

In such a way, the method of light scattering can be used for analysis of the effect of milk content and technological processes which are related to heating (pasteurization, sterilization, homogenization) on the size distribution of milk particles.

\subsection{SUMMARY}

Method of absorption/transmission spectroscopy of milk in the visible part of spectrum demonstrates a serious limitation - the milk sample should be adequately diluted to obtain the linear dependence of optical density on the concentration of milk sample which must be evaluated.

Method of infrared microspectrophotometry of milk is based on the measurement of the absorption of the main components of milk (fat, 


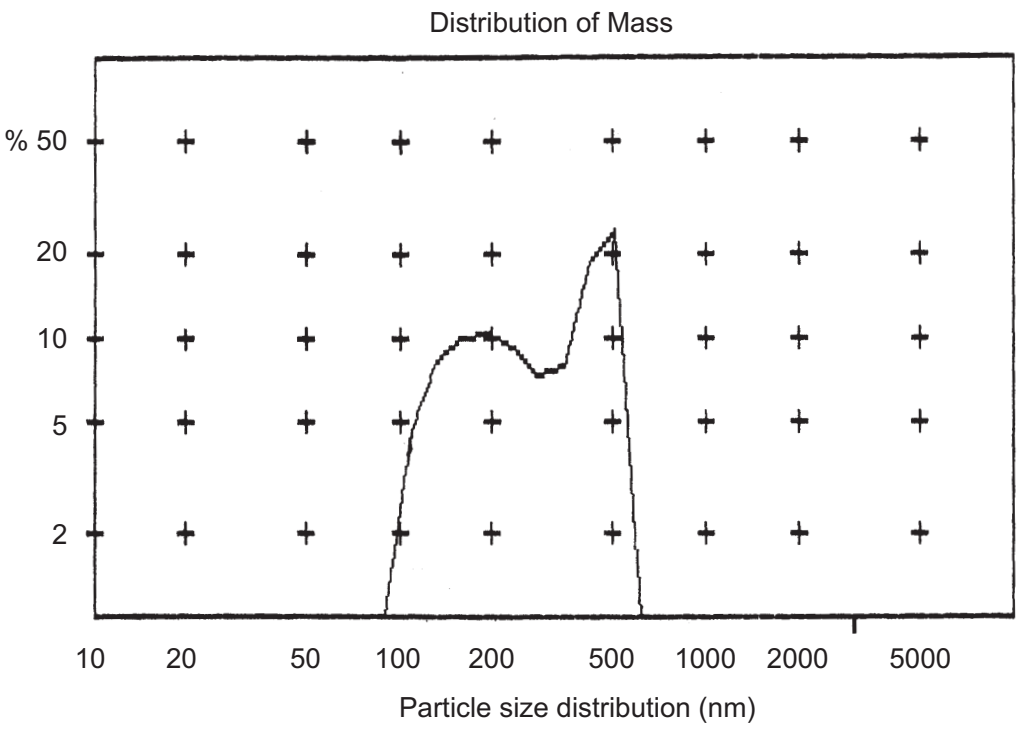

Fig. 4.59 Typical histogram of size distribution of the diluted $\left(\leq 10^{-3} \%\right)$ milk. Temperature $25^{\circ} \mathrm{C}$

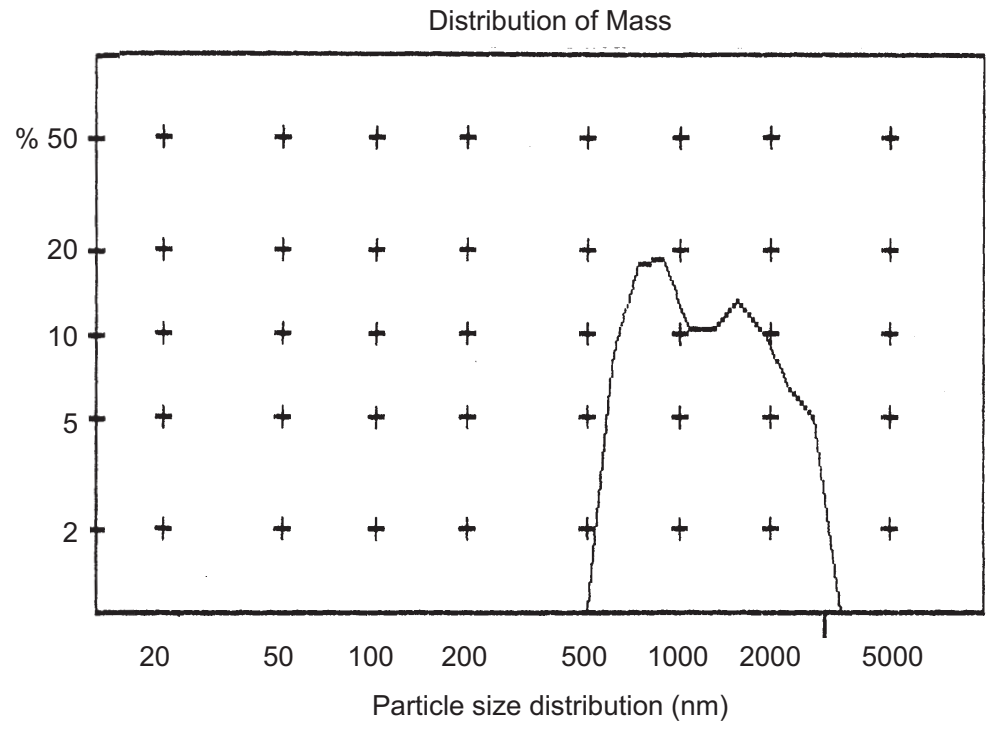

Fig. 4.60 Histogram of size distribution of milk. Concentration of fat $4.2 \%$; temperature $20^{\circ} \mathrm{C}$ 


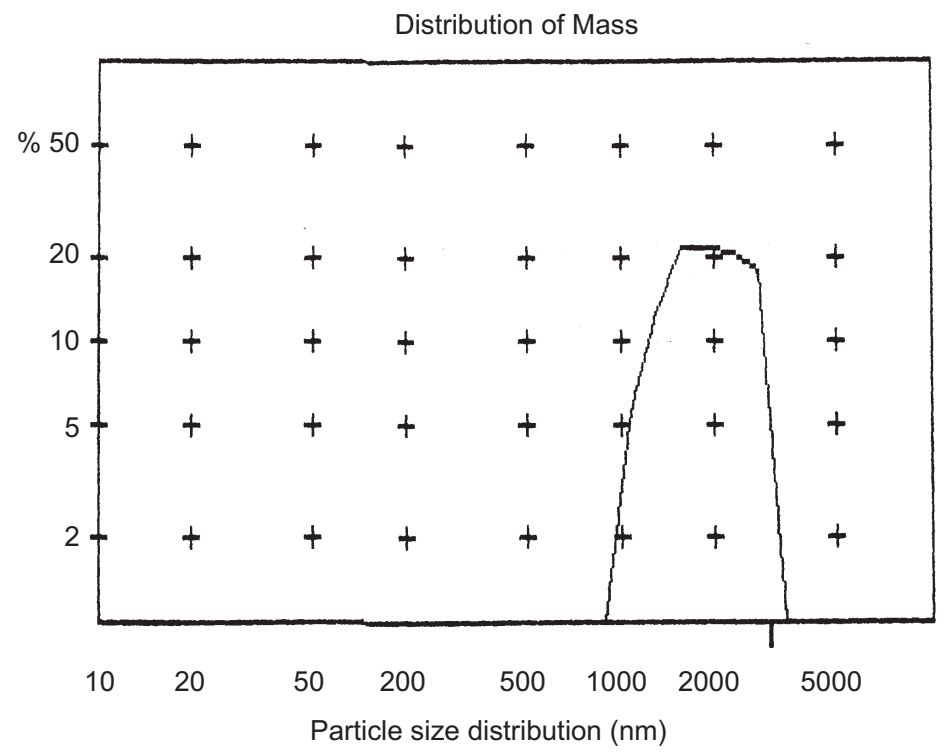

Fig. 4.61 Histogram of size distribution of milk. Concentration of fat 5.2\%; temperature $20^{\circ} \mathrm{C}$

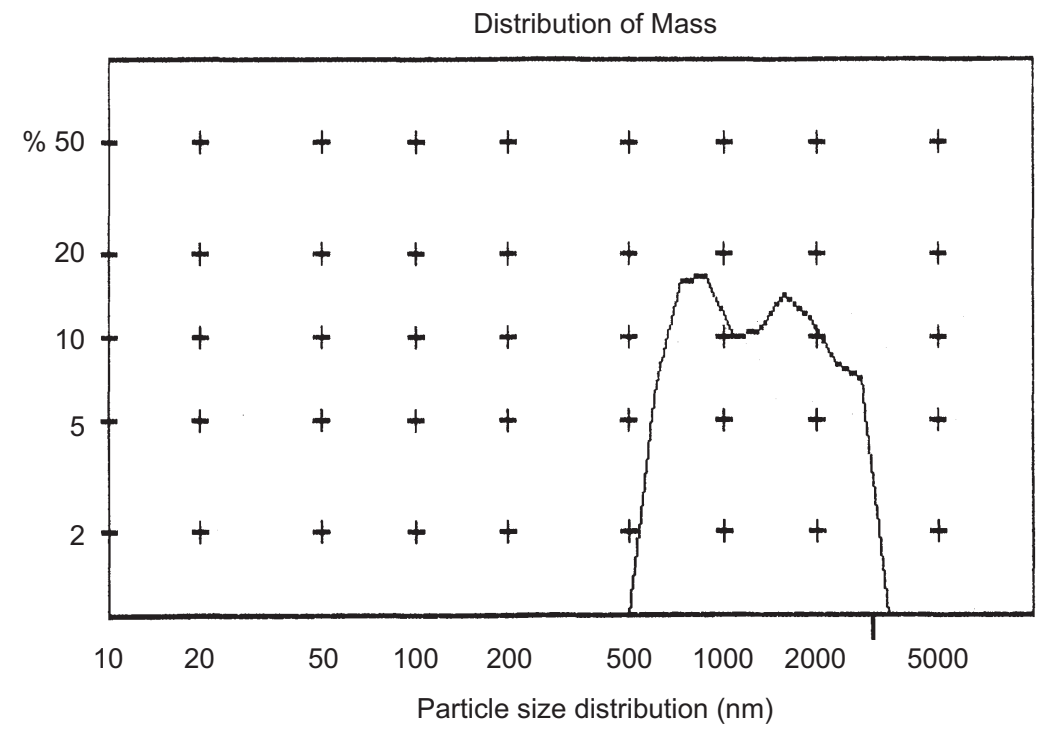

Fig. 4.62 Histogram of size distribution of milk. Concentration of fat $7.4 \%$; temperature $20^{\circ} \mathrm{C}$ 


\section{Practical Spectroscopy in Agriculture and Food Science}

protein, lactose) and quantitative evaluation of these components. The spectral position of infrared absorption bands depends on the temperature, level of milk homogenization, and $\mathrm{pH}$ of the milk.

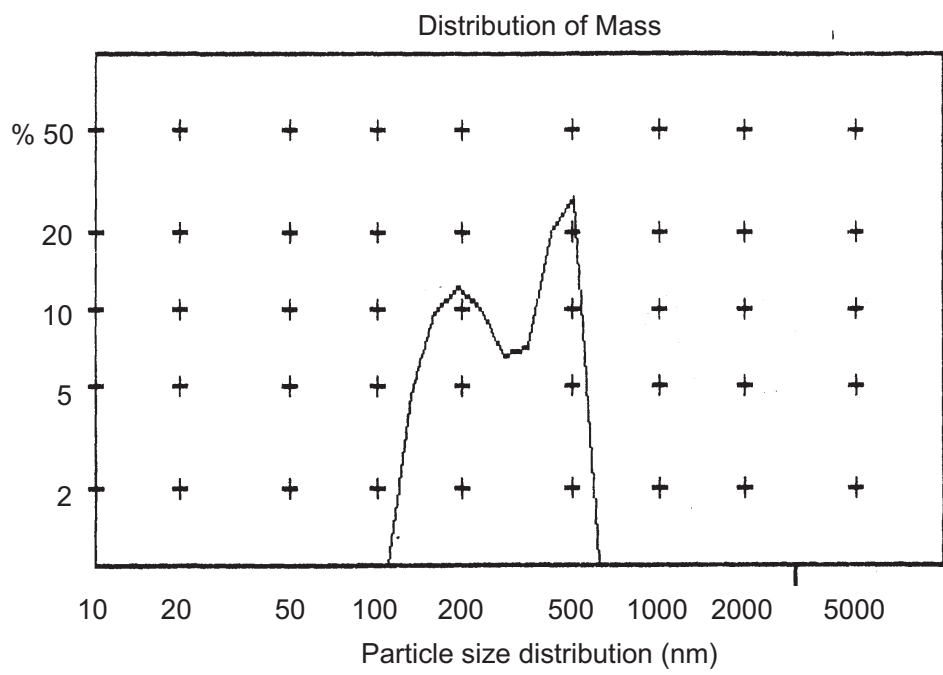

(a)

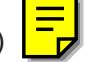

Fig. 4.63 Histogram of size distribution of milk. Concentration of fat $7.4 \%$; temperature $50^{\circ} \mathrm{C}$
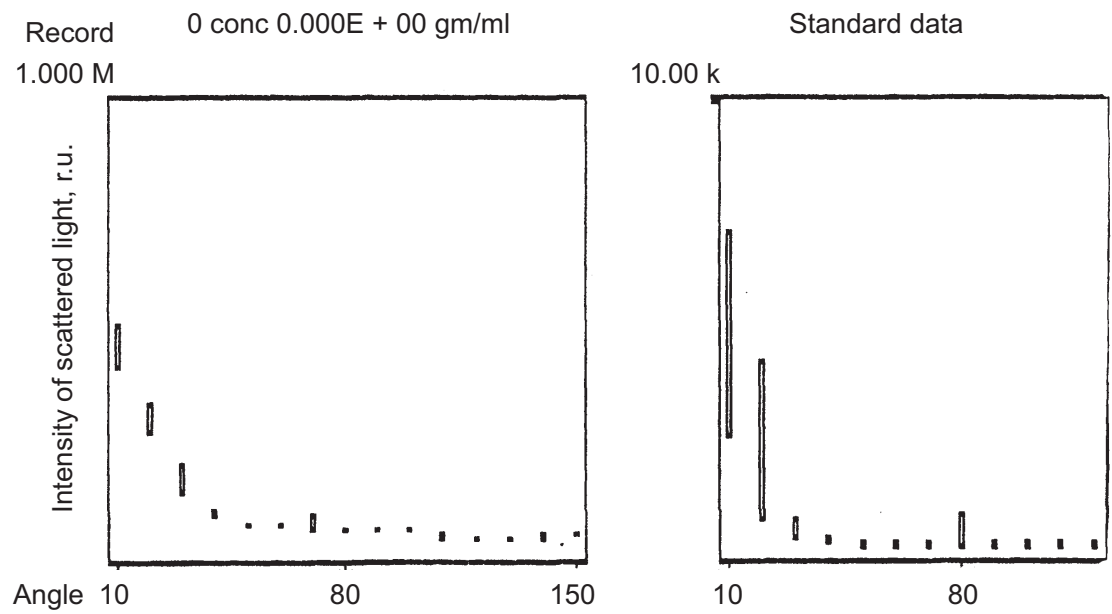

Fig. 4.64 Dependence of the intensity of scattered light on the angle of observation 
A number of milk components (proteins, fat, vitamins) demonstrate fluorescence. However, the fluorescence intensity of milk depends linearly on the concentration of the sample for diluted solutions only; the fluorescence method can be used in laboratory measurements.

The method of near-infrared spectroscopy provides the possibility to analyze milk samples which contain a high proportion of water and demonstrate high level of opacity. The NIR method is rapid, nondestructive and offers a high level of accuracy in comparison with traditional chemical methods.

The method of light scattering can be used for analysis of the size distribution of milk particles and the effect of technological processes which are related to heating (pasteurization, sterilization, homogenization) on this distribution.

\subsection{REFERENCES}

Biggs, D.A. and Sjaunja, L.O. 1987. Analysis of Fat, Protein, Lactose and Total Solids by Infra-Red Absorption. Bull. Int. Dairy Food. 208: 21-30.

Brusilovsky, L.P. and Waynberg, A.Ya. 1990. Devices of Technical Control in Dairy Industry. Moscow. p 287.

Fuhrman, I. 1987. Zur anwendung der nah-infrarotreflexions-spektroskopie in der milchwirtschaft. Lebensmit teilidustrie. 4: 171-174.

Goulden, J.D.S. 1964. Analysis of milk by infrared absorption. J. Dairy Res. 31: 273-284.

Hulst, H.C. 1982. Light Scattering by Small Particles. Dover Publ. Inc. p 470.

Krasnikov, V.V. and Timoshkin, E.I. 1983. Luminescence of Foods. Moscow, Light and Food Industry. p 264.

Krasnikov, V.V. and Timoshkin, E.I. and Titkova, A.V. 1987. Spectral Luminescence Analysis of Foods. Moscow, Agropromizdat. p 288.

Kucherov, A.P. 1984. Calculation of the values which characterise the overlapped spectral lines. J. Applied Spectroscopy. 41(1): 79-82.

Martynenko, I.I., Posudin Yu.I. and Shevchenko A.I. 1996. Infrared photometer. Food and Processing Industry. 2: 30.

Posudin, Yu.I. 1988. Lasers in Agriculture. Science Publishers, Inc. Enfield, NH, USA. p 188.

Posudin, Yu.I. 1993. Luminescence method of milk control. Isvestia VUZov. Food Technology. 5-6: 79-81.

Posudin, Yu.I. 1993. Turbidimetric methods of milk control. Isvestia VUZov. Food Technology 3-4: 85-87.

Posudin, Yu.I. 1995. Laser fluorometer for determination of fat in milk. Isvestia of VUZov. Food Technology 3-4: 72-73.

Posudin, Yu.I. 1996. Methods of Optical and Laser Spectroscopy in Agriculture. Seminar Series of visiting Fulbright Schol $\equiv$ niversity of Georgia, Athens, U.S.A. January-April 1996.

Posudin, Yu.I. 2002. Method of Determination of Carbohydrates in Biological Objects. Patent of Ukraine, 29114A, N 98010144, 2002. 


\section{Practical Spectroscopy in Agriculture and Food Science}

Posudin, Yu.I. 2005. Methods of Nondestructive Quality Evaluation of Agricultural and Food Products. Aristey, Kyiv. p 408.

Posudin, Yu.I. and Kostenko, V.I. 1989. Laser determines fat content. Agroprom of Ukraine. 4: 64.

Posudin, Yu.I. and Kostenko, V.I. 1991. Method of laser spectrofluorometry of milk and dairy products. Agr. Biology. 2: 191-195.

Posudin, Yu.I. and Kostenko, V.I. 1991. Method of determination of fat content in milk. Invention N 1698715 USSR.

Posudin, Yu.I. and Kostenko, V.I. 1992. Determination of milk content on the basis of Infrared spectrophotometry. Isvestiya VUZov. Food Technology. 3-4: 64-66.

Posudin, Yu.I. and Kostenko, V.I. 1994. Spectroscopy of Milk. Urozhaj, Kyiv. p 83.

Posudin, Yu.I., Martynenko, I.I. and Shevchenko, A.I. Instrument for Quantitative Determination of Milk Components. Patent of Ukraine, 14626 A. N95041858, 1997. 三

Posudin, Yu.I. and Timoshenko Yu. A. 1991. Modern Methods of Determination of Content. Agr. Academy Publ., Kiev. p 69.

Timoshkin, E.I. and Titkova, A.V. 1986. Spectral characteristics of luminescence of several vitamins. Moscow. Depon. VINITI. N 1551. B86.

Udenfriend, C. 1985. Fluorescence Analysis in Biology and Medicine. Moscow, Mir. p 484.

Voronkova, E.M., Grechushnikov, B.M., Distler, G.I. and Petrov, I.P. 1965. Optical Materials for Infrared Technique. Nauka, Moscow, Nauka. p 322.

Walstra, P. 1964. Approximation formulae for the light scattering coefficient of dielectric spheres. J. Appl. Puegs. 15(12): 1545-1552.

Walstra, P. 1965. Light scattering by milk fat globules. Neth. Milk Dairy J. 19(2): 93-109. 


\section{Spectroscopic Analysis of Eggs}

\subsection{PROPERTIES OF EGGS}

\subsubsection{Structure and Contents of the Egg}

The main parts of the egg are presented in Fig. 5.1. In the centre of the egg is the yolk which consists of latebra, germinal disc (blastoderm), and concentric light and dark layers, surrounded by the vitelline membrane. The albumen consists of the inner thick white (chalaziferous), inner thin white, thick white and outer thin white layers. The next layers are the inner and outer shell membranes. The thickness of these layers is about 0.01-0.02 mm [Stadelman and Cotterill, 1973]. All these elements are covered with the eggshell, which includes the cuticle, spongy and mammillary layers.

The main constituents of albumen are water (86-88\%) and total solids (12-14\%) which consist of proteins (9.7-10.6\%).

The yolk has $50 \%$ total solids, which include proteins (15.7-16.6\%) and lipids (31.8-35.5\%).

The eggshell consists of calcium carbonate (94\%), magnesium carbonate $(1 \%)$, calcium phosphate $(1 \%)$ and organic matter $(4 \%)$.

\subsubsection{Eggshell Colour and Pigmentation}

The colour of the eggshell can be explained as a means of camouflage and temperature control [Solomon, 1997]. The main pigments which are responsible for the colouration of the eggshell are shell porphyrins or ooporphyrins - cyclic compounds formed by the linkage of four pyrrole rings through methylene bridges. These pigments are presented by the protoporphyrin, uroporphyrin and coproporphyrin. 
82 Practical Spectroscopy in Agriculture and Food Science

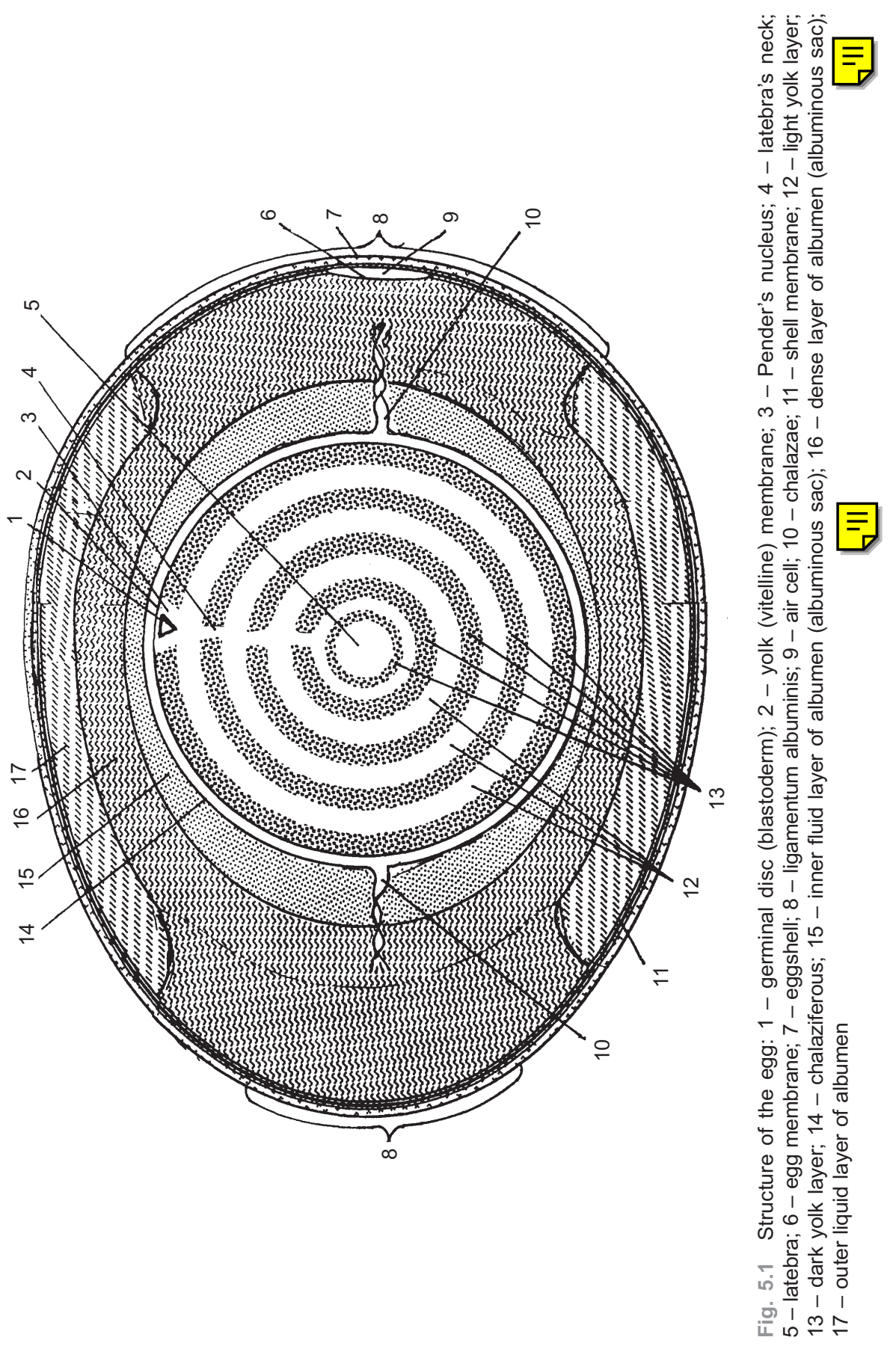




\subsection{CURRENT PROBLEMS OF EGG QUALITY EVALUATION}

The modern poultry industry is not satisfied with the traditional system of handling and processing of eggs, which is based on candling - visual inspection of the eggs. Currently, the operator of the conveyor does not have the opportunity to inspect 25,000 eggs per hour and estimate the freshness, weight, bacterial infection, presence of technical spoilage, and eggshell defects without elimination of subjectivity, fatigability and destruction. That is why automatization of egg quality control is rather actual.

Measures of egg quality. The main parameters of the egg which are inspected during its quality evaluation are: freshness of the egg, presence of the interior quality defects, weight, density and shape (egg index, asymmetry) of the egg, state of the eggshell, size of the air cell, albumen and yolk quality, Haugh unit, ratio of albumen weight to yolk weight, and the eggshell thickness.

Main interior quality defects in eggs. The most common defect found in eggs is related to blood spots; another type of internal spoilage is meat spots. The nature of blood and meat spots and factors are discussed by Nalbandow and Card (1944), and Helbaska and Swanson (1958). A less frequent defect is yolk mottling, which means a non-uniform distribution of water [Polin, 1957] or a separation of the vitelline membrane and the chalaziferous layer of the albumen [Doran and Muellar, 1961].

Eggshell defects. The most commonly detected surface defects and irregularities are [Solomon, 1997]: calcium splash, fine and heavy dusted, chalky deposit, lilac and pink eggs, body-cheched egg (equatorial bulge), white-banded and slab-sided eggs, corrugated eggs, and various mechanical defects (cracks, breakings, incisions).

\subsection{ABSORPTION/TRANSMISSION SPECTROSCOPY OF EGGS IN VISIBLE PART OF SPECTRUM}

Instrumental measurement of light transmission can be considered as an alternative to visual candling of the eggs. Spectrophotometry of shell colour [Brant et al., 1953], yolk colour [Philip et al., 1976], blood and meat spots [Brant, 1953] present the first attempt of the automation of egg quality control. The principle of blood and meat spots detection was based on the measurement of light transmission at $575 \mu \mathrm{m}$ where haemoglobin has the absorption band [Blackburn, 1964]. The disadvantage of this method is the dependence of the transmitted light 
on the size, colour of egg, sickness and contamination of the eggshell. The detection of the technical spoilage of the eggs on the basis of transmission spectra analysis was proposed by Mayorov et al. [In: Tzarikov and Chernova, 1968]. It was shown that fresh white eggs had the maximum transmission at 573-580 nm, brown eggs at 590-596 and 636-638 nm, and eggs with blood spots had absorption maxima at 538540 and $573-576 \mathrm{~nm}$.

The electronic ovoscope for egg quality grading was developed in a United Kingdom packing station [Wells and Belyavin, 1987]. This system consisted of high-resolution cameras which were capable of inspecting 48 eggs moving on a conveyor. The picture which was projected on a screen, allowed the operator to electronically label the suspected egg and remove it from the conveyor.

It is very attractive to use the spectrophotometry of eggs for the nondestructive determination of the fecundated eggs. The fact is that fecundated and nonfecundated eggs differ with the production of carbon dioxide as the result of egg metabolism (threefold according to Duley [In: Tzarikov and Chernova, 1968]. However, carbon dioxide is responsible for the absorption at $426.8 \mathrm{~nm}$; the measurement of the absorption of $\mathrm{CO}_{2}$ can make it possible to determine the fecundated eggs and to select them. Unfortunately, this problem has not yet been resolved.

\subsection{FLUORESCENCE SPECTROSCOPY OF EGGS}

The fluorescence characteristics of the eggs present a convenient quality criterion. Bogolubsky (1958) was the first to propose the application of visual estimation of eggs fluorescence to their selection; he introduced eight classes of colouration of eggshell during fluorescence emission.

The excitation of fluorescence by ultraviolet radiation was used for the quality evaluation of eggs with different levels of pigmentation [Podlegayev and Sarycheva, In: Tzarikov and Chernova, 1968; Van Deren and Jaworski, 1968]. The possibility to distinguish the eggs which were infected by Pseudomonas was also demonstrated.

The dependence of the fluorescence of eggs on the season, hen age, egg productivity and impregnation was investigated by Bekhtina and Dyagileva (1968).

The fluorescence excitation (with maxima at 405, 510, 540, and 575 $\mathrm{nm}$ ) and emission (635 and $700 \mathrm{~nm}$ ) were related to the pigments of the porphyrin nature - ptotoporphyrin IX and porphyrin derivatives of florin and oxoflorin [Rybalova and Maslova, 1985]. 
The detection of spoiled eggs on the basis of fluorescence measurements was proposed by Imai and Saito (1985). The intensity of fluorescence at 457 and $490 \mathrm{~nm}$ were used as spectral criteria of egg quality.

\subsection{DEFECTOSCOPY OF EGGSHELL}

The lighting methods (strobe and incandescent) were used for the acquisition of images and for the detection of cracks in eggs [Goodrum and Elster, 1992]. Strobe lighting appeared to be an acceptable alternative to incandescent light for machine vision inspection of light.

The laser scanning system as a means of detecting hair cracks in eggs was developed by Bol (1981).

The method of laser defectoscopy of eggs on the basis of the Faraday effect was proposed by Bezverkhny and Pigarev (1984). The egg on the conveyor is illuminated by laser radiation. Either incident or reflected radiation is polarized. The external magnetic field which is applied to the egg can rotate the plane of polarization of the reflected radiation. The angle of rotation depends on the state of eggshell, particularly on the mechanical defects. The system can respond to the intensity of reflected radiation and provide selection of the eggs.

Nondestructive evaluation by the holographic method of the strength of an egg was proposed by Vikram et al. (1980). The analysis of interference between reflected and reference laser beams made it possible to estimate the deformations and the strength of eggshell through the calculation of the interference bands.

\subsection{INSTRUMENTATION}

The absorption spectrometer consisted of a halogen lamp, monochromator, sample, photomultiplier and readout system. A signal $A=\left(I-I_{N}\right) /\left(I_{0}-I_{N}\right)$ was registered, where signals $I$ and $I_{0}$ were recorded with and without egg correspondingly, and $I_{N}$ was a level of noise which was estimated with closed photomultiplier.

Fluorescence properties of the eggshell were evaluated with luminescence microscope LUMAM-3M. The mercury lamp DRSh-250 1 was used as the source of fluorescence excitation; the radiation of this lamp passed through water infrared filter 2, glass filter 3, semitransparent plate 4 , and objective 5 of the microscope on the sample 6 . Fluorescence radiation was registered through the plate 4 and filter 7 by photomultiplier FEU-79 8 and readout system H-306 9 (Fig. 5.2). 


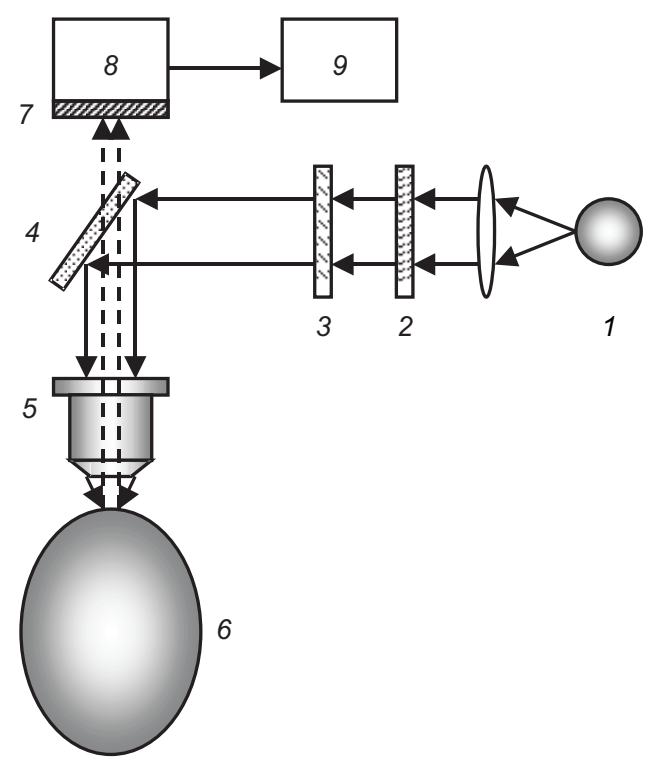

Fig. 5.2 An optical design of fluorometer for studying the fluorescence properties of the eggshell. 1 - mercury lamp DRSh-250; 2 - water infrared filter; 3 - glass filter; 4 - semitransparent plate; 5 - objective of the microscope; 6 - sample; 7 - filter; 8 - photomultiplier FEU-79; 9 - readout system H-306 [Posudin and Lepeshenkov, 1991; Posudin et al., 1992]

Fluorescence decay is an important parameter which can also be used for the quantitative estimation of eggshells. It was shown that the fluorescence decay process is described by two-exponential function [Posudin and Lepeshenkov, 1991; Posudin et al., 1992]:

$$
I(t)=A e^{-t / T_{1}}+B e^{-t / T_{2}}
$$

where $I(t)$ is intensity of fluorescence, $A$ and $B$ are constants; $T_{1}$ and $T_{2}$ - decay time of both exponential curves, $t$ - time, $e$ - base of the natural logarithm.

Laser defectoscopy of the eggshell was based on the interaction of laser radiation that passes into objective of the microscope through the eggshell cracks during rotation of the egg. The reflected laser beam will change its intensity and direction; alterations of the reflected beam give the information about crack parameters. The typical laser defectoscope consists of laser 1, mirror 2, microscope objective 3, egg sample 4, photodetector 5, and readout system 6 (Fig. 5.3). 


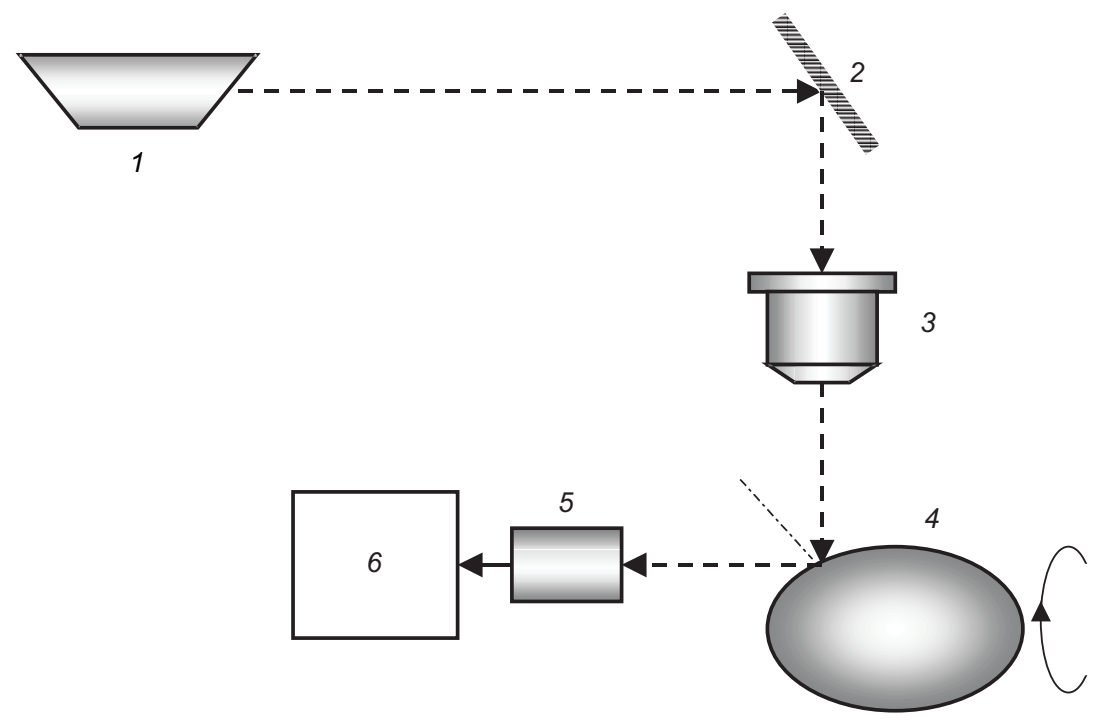

Fig. 5.3 Principles of laser defectoscopy of the eggshell. 1 - laser; 2 - mirror; 3 microscope objective; 4 - egg sample; 5 - photodetector; 6 - readout system [Posudin and Lepeshenkov, 1991]

\subsection{RESULTS OF SPECTROSCOPIC ANALYSIS OF EGGS AND EGGSHELLS}

\subsubsection{Reflectance Spectra}

The dependence of factor such as $1-I_{s h} / I_{s t}$ on the wavelength $\lambda$ was estimated as reflectance spectrum of the eggshell of white and brown colour, where $I_{s h}$ and $I_{s t}$ are intensities of light reflected from the eggshell and standard (chalky paper) correspondingly. These reflectance spectra which demonstrate the maxima at 480, 535, 563, 590 and $642 \mathrm{~nm}$, are presented in Fig. 5.4 [Posudin and Lepeshenkov, 1991, 1992]. The intensity of reflected light is different for white and brown eggshells.

\subsubsection{Transmission Spectra}

The typical spectra of transmission of the hen and duck eggs are presented in Fig. 5.5 [Posudin et al., 1992, 1995, 1998, 2005]. It is possible to distinguish the spectra of the fresh eggs (1), eggs with blood inclusions (2), and spoiled eggs (3). The transmission peak for fresh white hen egg is located at 573-580 nm; the spectra of cream fresh eggs have two peaks at $590-596 \mathrm{~nm}$ and $636-638 \mathrm{~nm}$. It is clear that the spectral position of transmission maximum of eggs at 550-600 nm 


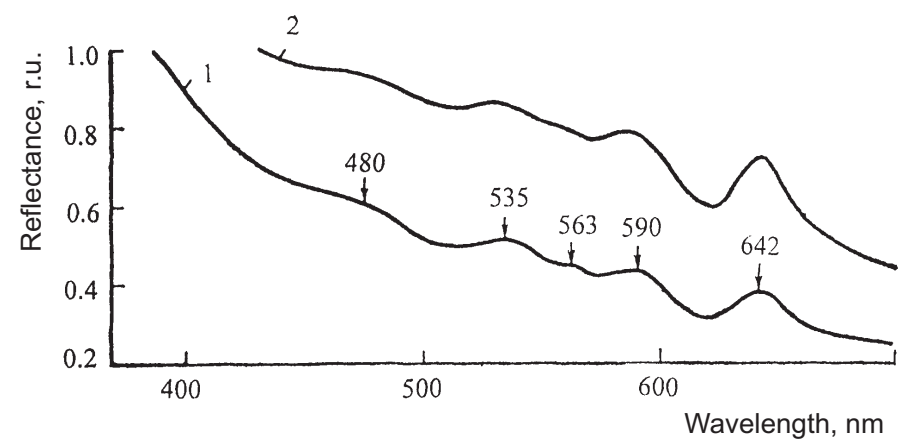

Fig. 5.4 Reflectance spectra of eggshell: 1 - light cream colour; 2 - dark cream colour [Posudin and Lepeshenkov, 1991]

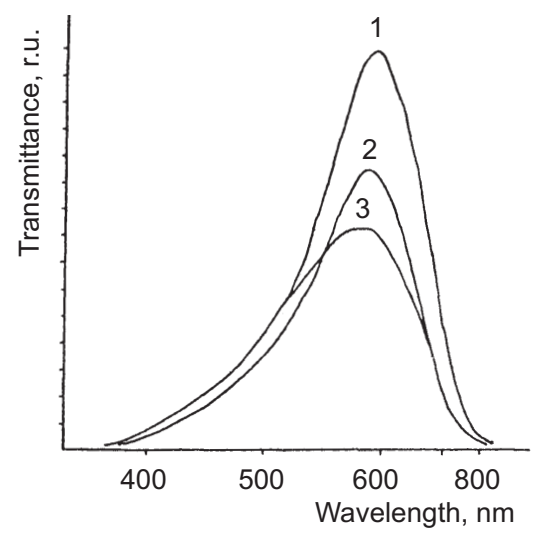

Fig. 5.5 The typical spectra of transmission of the hen and duck eggs. 1 - fresh eggs; 2 eggs with blood inclusions; 3 - spoiled eggs [Posudin and Lepeshenkov, 1991, 1992; Posudin et al., 1992]

depends on the freshness of the eggs and presence of technical spoilage [Posudin and Lepeshenkov, 1991, 1992; Posudin et al., 1992].

\subsubsection{Fluorescence Spectra}

The fluorescence spectroscopy is a rather promising approach for the detection of different types of spoilage and quantitative estimation of eggshell pigmentation [Posudin et al., 1992]. The typical spectra of fluorescence emission of hen and duck eggs, fresh and spoiled, with different eggshell colour are presented in Fig. 5.6. The intensity of the main maximum at $672-674 \mathrm{~nm}$ depends on the freshness of the eggs, level of eggshell pigmentation, and the interior defects. 


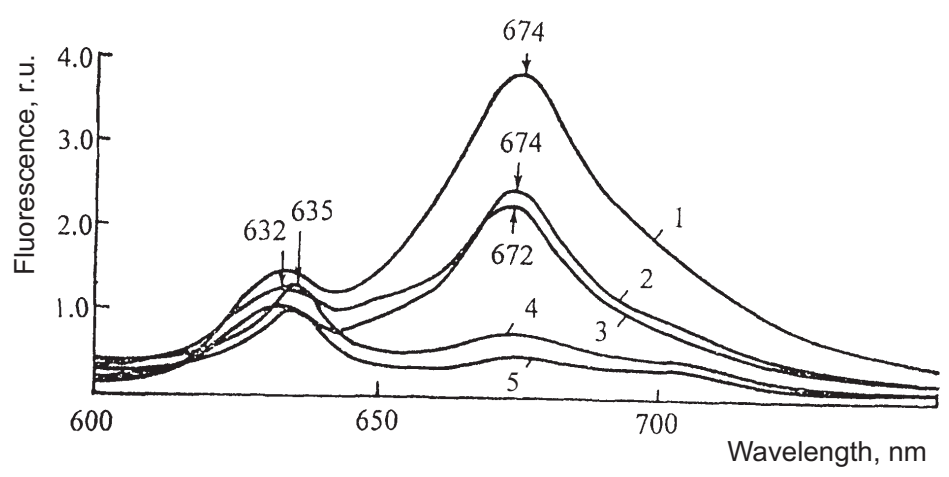

Fig. 5.6 Typical spectra of fluorescence emission of hen and duck eggs, fresh and spoiled, with different eggshell colour. 1-3 - hen eggs; 4-5 - duck eggs; 1 - dark cream colour; 2, 3 - light cream colour; 5 - eggs with blood inclusions [Posudin et al., 1992]

The typical fluorescence decay curves for the eggs with different levels of pigmentation are presented in Fig. 5.7 [Posudin et al; 1992; Posudin and Kucherov, 1992]. Here the level of pigmentation is decreasing from N1 to N7. The quantitative data of the fluorescence decay are presented in Table 5.1.

Table 5.1 Amplitude and temporal constants of exponents

\begin{tabular}{c|c|c|c}
\hline \multicolumn{2}{c|}{ Amplitude of Exponents } & \multicolumn{2}{c}{ Temporal Constants of Exponents } \\
\hline$A$ & $B$ & $\tau_{1}$ & $\tau_{2}$ \\
\hline 82.297 & 21.181 & 636.224 & 17.567 \\
81.932 & 41.596 & 621.063 & 38.838 \\
66.256 & 32.713 & 413.558 & 14.777 \\
45.246 & 22.350 & 629.568 & 21.525 \\
92.594 & 43.872 & 598.981 & 26.776 \\
60.115 & 49.165 & 369.775 & 10.948 \\
35.627 & 33.817 & 216.245 & 7.665 \\
\hline
\end{tabular}

\subsubsection{Defectoscopy of Eggshell}

Laser radiation with a small divergence can be applied to the detection of eggshell cracks. The minimal spot diameter of laser radiation after its focusing by a microscope is determined by the numerical aperture, wavelength of light and focal distance of the objective [Posudin and Lepeshenkov, 1991, 1992; Posudin et al., 1992]:

$$
D_{\min }=2 \omega f \text {. }
$$




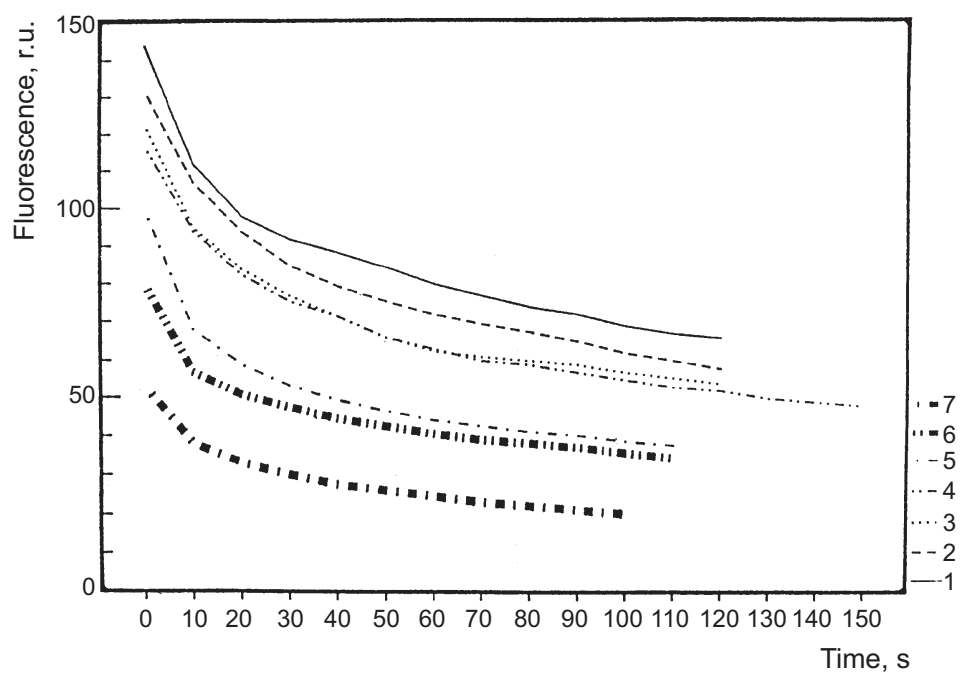

Fig. 5.7 Typical fluorescence decay curves for the eggs with different levels of pigmentation. Here the level of pigmentation is decreasing from N1 to N7 [Posudin et al., 1992; Posudin and Kucherov, 1992]

Combination of He:Ne laser $\left(\omega=5 \cdot 10^{-4} \mathrm{rad}, \lambda=632 \mathrm{~nm}, P=55 \mathrm{~mW}\right)$ with the objective $8 \times 0.20$ (focal distance $f=18.2 \mathrm{~mm}$ ) gives

$$
D_{\text {min }}=2.5 \cdot 10^{-4} 18.2 \cdot 10^{-3}=18.2 \mu \mathrm{m}
$$

and with the objective $40 \times 0.65$ (focal distance $f=4.25 \mu \mathrm{m}$ )

$$
D_{\min }=2.5 \cdot 10^{-4} 4.25 \cdot 10^{-3}=4.25 \mu \mathrm{m}
$$

These values of $D_{\min }$ can be considered as threshold diameters of laser radiation which can be realized during defectoscopy of the eggshell.

Further progress in the egg quality practice is related to the development of automated instrumentation of control, such as image analysis system. The main procedure in these systems is the scanning of the object to assess its size and shape. The information is elaborated by a microprocessor and is used for the sorting of eggs.

\subsection{SUMMARY}

Spectroscopic methods can be applied to non-destructive evaluation of poultry-farming products. The intensity of reflected light is different for white and brown eggshells. The maximum spectral position of 
transmission of eggs depends on the freshness of the eggs and presence of technical spoilage. The intensity of fluorescence maxima depends on the freshness of the eggs, level of eggshell pigmentation, and the interior defects. Laser radiation with a small divergence can be applied to the detection of eggshell cracks.

\subsection{REFERENCES}

Bekhtina, V.G. and Dyagileva, G.E. 1968. Fluorescence of eggs and economically useful signs of hens. In: Biological basis of breeding and artificial insemination of livestocks (Russian), Leningrad. 12(2): 154-160.

Bezverkhny, S.A. 1985. Agricultural Professions of Laser Beam (Russian), Agropromizdat, Moscow, p 135.

Bezverkhny, S.A. and Pigarev, L.A. 1984. Laser defectoscopy of eggs. Doklady. VASKHNIL (Russian) 4: 38-39.

Blackburn, W.E., Bryant, T.D. and Harris, D.I. 1964. Apparatus for detecting blood spots in eggs. Patent USA No 3130255, 21 April 1964.

Bogolubsky, S.I. 1958. Fluorescence of eggs as selection sign. Proc. of Pushkin Sci. Laboratory of Poultry-Breeding, Pushkin, Russia (or USSR), V.8.

Bol, J. 1981. Laser scanning system for the automated inspection of eggs for haircracks. In: Quality of Eggs, Proc. of 1st European Egg Quality Symposium (Group 4), G.M. Beuving, C.W. Scheele, P.C.M. Simons, eds. Beekbergen, Netherland, Spelderholt Institute of Poultry Research. p. 84-93.

Brant, A.W. 1953. Machine sorts eggs for shell colour. USDA. Poultry Processing and Marketing. 59: 12-13.

Brant, A.W., Norris, K.H. and Chin, G. 1953. A spectrophotometric method for detecting in white-shelled eggs. Poultry Science 32: 357-363.

Doran, B.M. and Muellar, W.J. 1961. The development and structure of the vitelline membrane and their relationship to yolk mottling. Poultry Sci. 40: 474-478.

Goodrum, J.W. and Elster, R.T. 1992. Machine vision for crack detection in rotating eggs. Transactions of the American Society of Agricultural Engineers 35(4): 1323-1328.

Helbaska, A.V.I. and Swanson, M.H. 1958. Studies on blood and meat spots in the hen's egg. 2. Some chemical and histological characteristics of blood and meat spots. Poultry Sci. 37: 817-885.

Imai, C. and Saito, J. 1985. Detection of spoiled eggs using a new-type black light egg inspection unit. Poultry Sci. 64(10): 1891-1899.

Nalbandow, A.V. and Card, L.E. 1944. The problem of blood clots and meat spots in chicken eggs. Poultry Sci. 23: 170-180.

Philip, T., Weber, C.W. and Berry, J.W. 1976. Rapid measurement of egg yolk color. Food Technol. 30(11): 58-59.

Polin, D. 1957. Biochemical and weight changes of mottled yolks in eggs from hens fed Nicarbazin. Poultry Sci. 36: 831-835.

Posudin, Yu.I. 1988. Lasers in Agriculture. Science Publishers, Inc. Enfield, NH, USA. p 188.

Posudin, Yu.I. 1995. Biophysics. Urozhaj, Kyiv. p 222.

Posudin, Yu.I. 2005. Methods of Nondestructive Quality Evaluation of Agricultural and Food Products. Aristey, Kyiv. p 408.

Posudin, Yu.I. and Kucherov, A.P. 1992. Method of control of eggs quality. Invention N 1783390 USSR, 1992. 
Posudin, Yu.I. and Lepeshenkov, V.F. 1991. Optical and laser methods of poultry-breeding control. St.-Petersburg Agr. Univ. Publ. 1: 59-64.

Posudin, Yu.I. and Lepeshenkov, V.F. 1992. Method of eggs quality evaluation. Invention N 1735766 USSR, 1992.

Posudin Yu.I., Tsarenko P.P. and Tsyganjuk, O.V. 1992. Eggs quality evaluation with optical and laser methods. Agr. Biology. 4: 69-74.

Rybalova, N.B. and Maslova, V.G. 1985. Visual and objective methods of estimation of eggshell luminescence. In: Development of methods which lead to increasing of poultry production. Leningrad. p. 22-29.

Solomon, S.E. 1997. Egg and Eggshell Quality. Iowa State Press, p 149.

Stadelman, W. J. and Cotterill, O.J. 1973. Egg Science and Technology. Westport, Conn, Avi Pub. Co., p 590.

Tzarikov, N.N. and Chernova, G.G. 1968. Application of physical methods in egg product industry. Central Institute of Information, Technical and Economical Investigations. Moscow, pp. 1-18.

Van Deren, J.M. and Jaworski, E.G. 1968. Collaborative study of the determination of exthoxyquin in chick tissue and eggs by fluorescence. J. of A.O.A.C. 51: 537-539.

Vikram, C.S., Vedam, K. and Buss, E.G. 1980. Nondestructive evaluation of the strength of eggs by holography. Poultry Sci. 59: 2342-2347.

Wells, R.G. and Belyavin, C.G. 1987. Egg Quality - Current Problems and Recent Advances. Proc. Poultry Science Symp. London, Boston, Durban, Sydney, Toronto, Wellington. N 20. $\mathrm{p} 302$. 


\section{Spectroscopic Analysis of Honey}

\subsection{PROPERTIES OF HONEY}

\subsubsection{Definition of Honey}

Honey, according to the accepted definition [Codex..., 1969], is the natural sweet substance produced by honey bees from the nectar of plants (Blossom Honey or Nectar Honey), or from secretions of living parts of plants, or excretions of plant sucking insects on the living parts of plants (Honeydew Honey), which the bees collect, transform by combining with specific secretions of their own, deposit, dehydrate, store and leave in the honeycomb to ripen and mature. Honey is a product with complex chemical composition: it contains plant pigments (carotenes, xanthophylls, chlorophyll), mineral substances, sugars, and various impurities.

Physical properties of honey are related to its state, age, presence of water and level of crystallization. All these factors affect the quality of honey.

\subsubsection{Composition of Honey}

The main components of honey are sugars, which are presented by fructose $(37.20 \%)$, glucose $(31.28 \%)$, sucrose $(1.31 \%)$, maltose $(7.31 \%)$, etc. [Je' Anne, 1991]. Blossom honey differs from honeydew by the values of simple sugars, disaccharides, higher sugars, acids, mineral salts and nitrogen content. The concentration of sugars can be used as a criterion of honey adulteration, which is provoked with the artificial addition of syrup, sucrose that is hydrolyzed with acids, starch or beetroot treacle in honey [Chudakov, 1967]. That is why precise quality evaluation of honey has long been the goal of many investigators and specialists who 


\section{Practical Spectroscopy in Agriculture and Food Science}

are related to honey-breeding [Vorwohl, 1984, 1990; Gonnet, 1986; Vakhonina et al., 1987; Dustmann, 1993; Mautz, 1993; Campos, 1994]. The composition of honey also reflects the contaminants which are present in the area of bee activity.

\subsection{SPECTROSCOPIC ANALYSIS OF HONEY}

Development of nondestructive methods, which are based on the analysis of the sample without any alterations of product attributes, present the problem of great practical significance. Spectroscopic methods occupy an important place among the comprehensive tests [Posudin, 2005]. These methods include the measurement of difference between input and output light signals during interaction of light with the sample (absorption, transmission, reflection, scattering, re-emission) and analysis of the dependence of this difference on the wavelength. Besides, spectroscopic methods are rather fast and precise. Effects of honey type, age, temperature, water content and degree of sugar adulteration on the spectral properties of honey can also be studied.

Certain spectroscopic methods that have been applied to honey control are mentioned in the literature: spectrophotometry [Yao and Chen, 1985; Yao and Fan, 1985; Salinas et al., 1994], optical activity measurement [Juan et al., 1992], atomic spectroscopy [Petrovic et al., 1994; Salinas et al., 1994]. However, the fact is that honey presents a nontransparent and opaque substance; the application of the abovementioned methods requires either the dilution, or special preparation of the samples. That is why it is necessary to use other spectroscopic methods which are based on the interaction of optical radiation with surface layers of the sample.

Two methods can be chosen from this point of view - fluorescence spectroscopy and reflectance spectroscopy in near-infrared (NIR) part of the spectrum. These methods can effectively provide nondestructive quality evaluation of honey.

The main objective of this investigation is development of the spectrum, based on fluorescence and NIR spectroscopy, approach to nondestructive quality evaluation of honey and establishment of the correlation between those factors which determine honey quality and its spectral properties.

\subsection{HONEY SAMPLES}

The samples of honeydew, which were used in these experiments, were taken from the collection of Bavarian Institute of Bee-Breeding 
(Erlangen, Germany)*, and the samples of honey - from different parts of Ukraine: Kiev, Dnepropetrovsk, and Crimea. Samples with different percentage of sucrose were prepared in the National Agricultural University for obtaining useful information about adulteration of honey. Descriptions of all the samples are given in Table 6.1. The measurements of spectroscopic properties of Bavarian "Tanne" samples were performed in 1993, and of the Ukrainian honey samples in 1994.

Table 6.1 Samples of honey which were used in the experiments

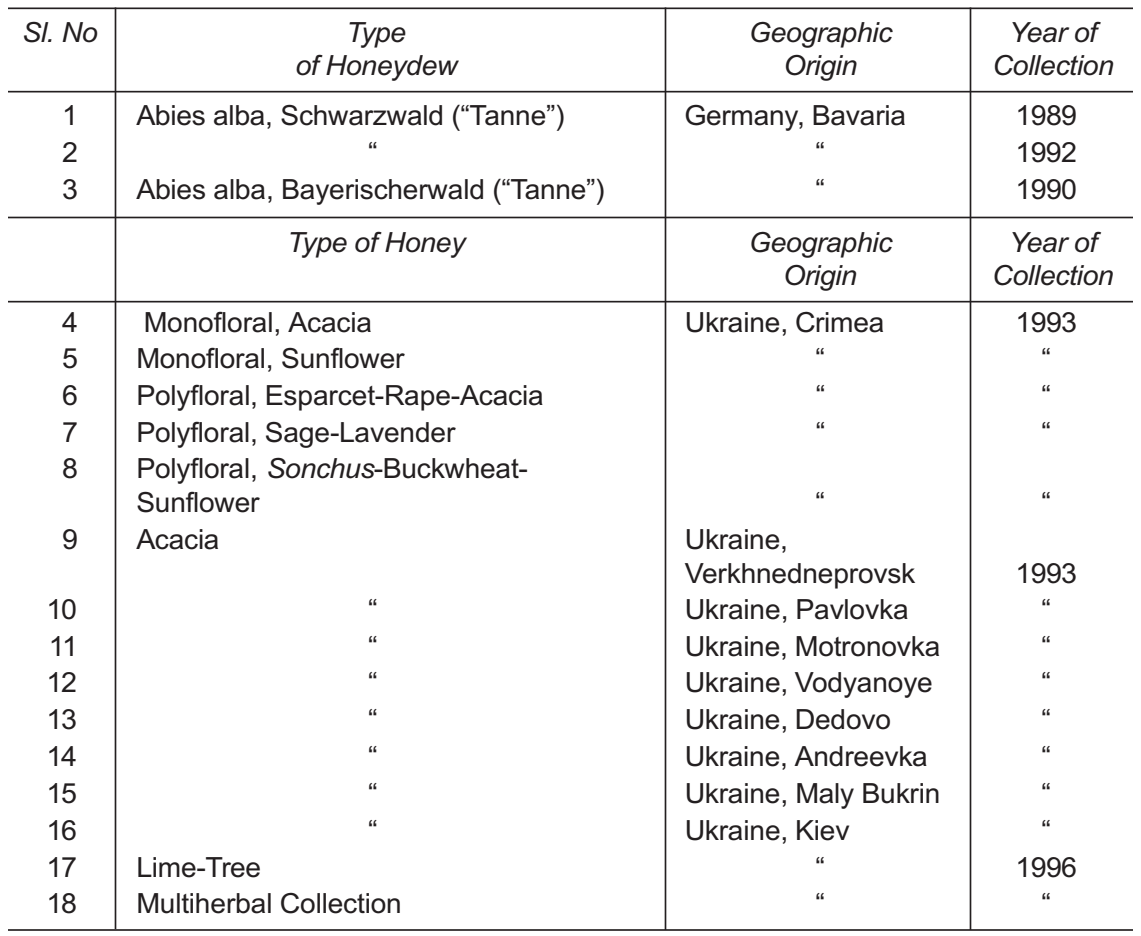

\subsection{INSTRUMENTATION}

The spectra of absorption and reflection of honey in the ultraviolet and visible part of the spectrum were measured with spectroscopic complex KSVU-23 ("LOMO", Russia), which was equipped with a double monochromator, diffraction gratings, and computer.

The investigation of fluorescence spectra of honey was performed with the spectrofluorometer SDL-2 ("LOMO", Russia) in the regime of

\footnotetext{
*The samples of honeydew were presented by the courtesy of Dr. D. Mautz.
} 
photon counting. The spectral range during these measurements was $200-700 \mathrm{~nm}$; the errors of measurement were $2 \mathrm{~nm}$ for intensity of the bands and $5 \mathrm{~nm}$ for half-width of the bands.

The reflectance spectra in the near-infrared (NIR) part of spectrum $(1,620-2,320 \mathrm{~nm})$ were measured with analyzer Model 4250 ("Pacific Scientific", USA). This analyzer has three ranges: 1,620-1,800 nm, 1,890$2,115 \mathrm{~nm}$, and 2,050-2,320 $\mathrm{nm}$. Reproducibility of the results was better than $0.015 \mathrm{~nm}$. The NIR spectrum was estimated as the dependence of optical density $D=\lg (l / R)$ on the wavelength $\lambda$ (where $R$ is reflection coefficient). All measurements were performed at room temperature.

\subsection{RESULTS OF SPECTROSCOPIC ANALYSIS OF HONEY}

The absorption spectra of honeydew ("Tanne") in ultraviolet and visible parts of the spectrum are presented in Fig. 6.1. A certain shoulder near $250-275 \mathrm{~nm}$ is standing out against the background which is decreasing monotonously from 200 to $700 \mathrm{~nm}$. The presence of the shoulder in absorption (reflectance) spectra of honey testifies the participation of several honey components in formation of absorption (reflectance) spectra in the ultraviolet and visible parts of the spectrum. The intensity of absorption can be used as a criterion of geographic origin or age of honey.

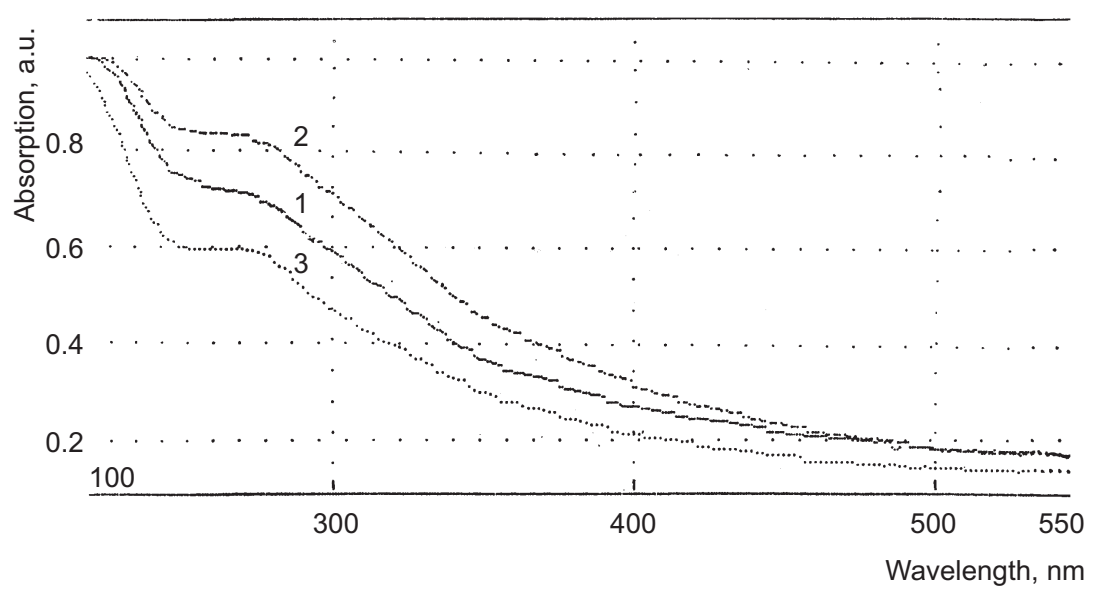

Fig. 6.I Absorption spectra of honey in ultraviolet and visible parts of spectrum. 1 Schwarzwald "Tanne", 1989; 2 - Schwarzwald "Tanne", 1992; 3 - Bayerischer "Tanne", 1990 [Posudin et al., 1995] 
The excitation fluorescence spectra of honeydew were investigated for different wavelengths of fluorescence emission $(440 \mathrm{~nm}, 560 \mathrm{~nm}$, and $575 \mathrm{~nm})$.

The emission fluorescence spectra of honeydew are characterized by a broad band (about 100-150 nm); the spectral position of its maximum depends on the wavelength of excitation. These maxima are located near $420 \mathrm{~nm}\left(\lambda_{\mathrm{exc}}=350 \mathrm{~nm}\right), 480 \mathrm{~nm}\left(\lambda_{\mathrm{exc}}=400 \mathrm{~nm}\right)$, and $510 \mathrm{~nm}\left(\lambda_{\mathrm{exc}}=450\right.$ $\mathrm{nm})$. The maximal intensity of emission takes place during excitation in the ultraviolet part of the spectrum. The fluorescence intensity of honeydew depends on the geographical origin and its age. Typical fluorescence emission spectra of honeydew are presented in Fig. 6.2.

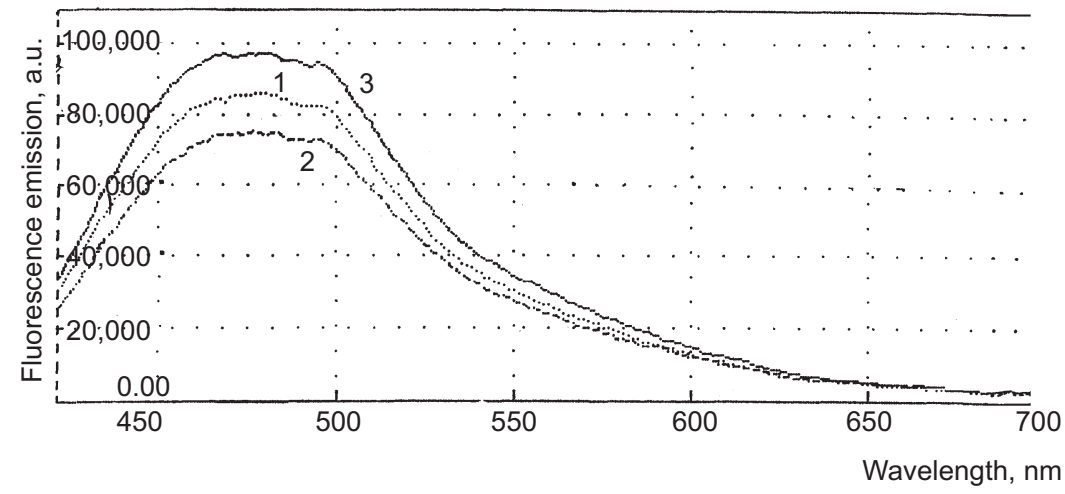

Fig. 6.2 Fluorescence emission spectra of honey (samples N1-N3). Wavelength of excitation $400 \mathrm{~nm}$ [Posudin et al., 1995]

The fluorescence intensity of honey also depends on the type of honey (Table 6.2); the samples of the same type of honey (Acacia), which were collected from different parts of one region and of one age, demonstrated quasi equal intensity of fluorescence, but this intensity depends on the age of honey (Table 6.3).

Table 6.2 Dependence of fluorescence intensity of honey on the geographic origin

\begin{tabular}{c|l|c}
\hline $\begin{array}{c}\text { SL. No. of Sample } \\
\text { (From Table 6.1) }\end{array}$ & \multicolumn{1}{|c}{ Type of Honey } & $\begin{array}{c}\text { Fluorescence } \\
\text { Intensity }\end{array}$ \\
\hline 4 & Monofloral, Acacia & $0.29 \pm 0.064$ \\
5 & Monofloral, Sunflower & $0.48 \pm 0.088$ \\
6 & Polyfloral, Esparcet-Rape-Acacia & $0.38 \pm 0.031$ \\
7 & Polyfloral, Sage-Lavender & $0.69 \pm 0.096$ \\
8 & Polyfloral, Sonchus-Buckwheat-Sunflower & $0.43 \pm 0.045$ \\
\hline
\end{tabular}


Table 6.3 Dependence of fluorescence intensity on the geographic origin and year of collection of honey

\begin{tabular}{c|c|c|c}
\hline $\begin{array}{c}\text { SL. No. of Sample } \\
\text { (From Table 6.1) }\end{array}$ & $\begin{array}{c}\text { Geographic } \\
\text { Origin }\end{array}$ & $\begin{array}{c}\text { Year } \\
1993\end{array}$ & $\begin{array}{c}\text { Year } \\
1994\end{array}$ \\
\hline 9 & Verkhnedneprovsk & $0.30 \pm 0.08$ & $0.53 \pm 0.07$ \\
10 & Pavlovka & $0.21 \pm 0.05$ & $0.68 \pm 0.07$ \\
12 & Vodyanoye & $0.31 \pm 0.09$ & $0.82 \pm 0.16$ \\
13 & Dedovo & $0.34 \pm 0.10$ & $0.44 \pm 0.03$ \\
14 & Andreevka & $0.28 \pm 0.09$ & $0.50 \pm 0.02$ \\
15 & Maly Bukrin & $1.28 \pm 0.06$ & $2.05 \pm 0.09$ \\
\hline
\end{tabular}

The fact of the dependence of intensity, half-width, and spectral position of fluorescence spectra on the wavelength of excitation and emission means the participation of several fluorophors in formation of fluorescence spectra of honey.

The effect of temperature on the fluorescence intensity of honey studied is demonstrated in Fig. 6.3. It is shown that the increasing of temperature provokes the decreasing of the fluorescence intensity.

The correlation between fluorescence properties of honey and the presence of water in it was also investigated. The quantity $W$ of water was estimated with the refractometric method according to the following relation [Aganin, 1989]:

$$
W=400\left[1.538-n\left(20^{\circ} \mathrm{C}\right)\right]
$$

where $n$ is the coefficient of refraction. The results of these measurements are presented in Table 6.4.

The coefficients of correlation were calculated for polyfloral honey $(r=-0.96)$ and monofloral honey $(r=-0.87)$. This strong negative correlation between the presence of water in honey and fluorescence intensity can be used for quality evaluation of honey.

The reflectance spectra of honey samples in NIR part of the spectrum are characterized by a number of reflectance bands near 1,779 nm, 1,933 $\mathrm{nm}$, and 2,290 nm; the relative intensity of the spectral bands depends on the type and age of the sample (Fig. 6.4). The samples taken from different parts of the same geographic region produced the same shape of the reflectance spectra, which were distinguished by the intensity of spectral bands only (Figs. 6.5 and 6.6). However, the samples that were taken from different geographic zones demonstrated different shapes (Figs. 6.4 and 6.5). 


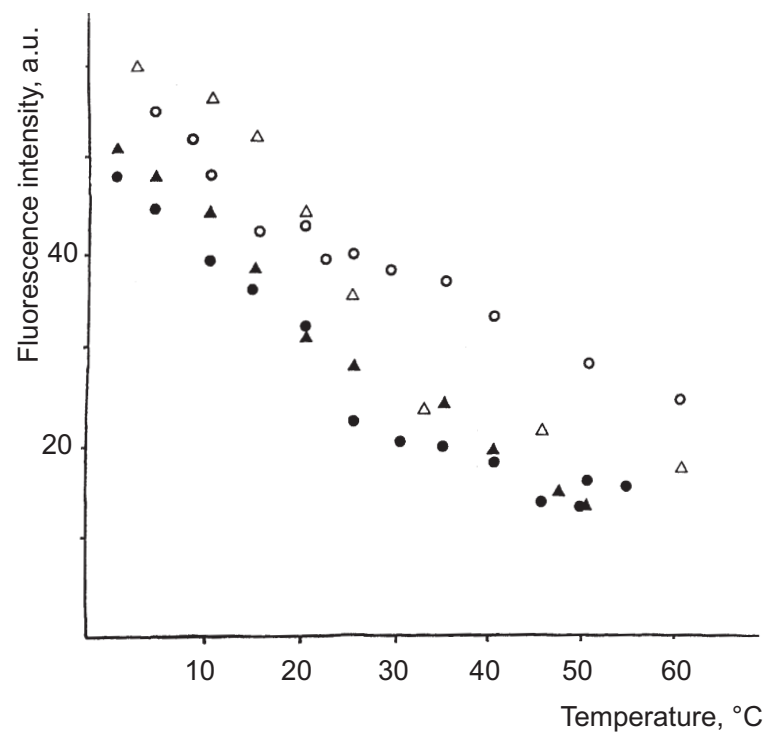

Fig. 6.3 Dependence of fluorescence intensity of honey on the temperature. - O- monofloral, acacia, sample N4; - $\Delta--$ monofloral, sunflower, sample N5; $-\Delta--$ polyfloral, sage-lavender, sample N7; -•- - polyfloral, sonchus-buckwheat-sunflower, sample N8 [Posudin et al., 1995]

Table 6.4 Results of estimation of quantity $W$ of water by fluorescence and refractometric methods

\begin{tabular}{c|c|c|c|c|c}
\hline $\begin{array}{c}\text { Index of } \\
\text { Refraction } \\
(N)\end{array}$ & $\begin{array}{c}\text { Quantity } \\
\text { of Water } \\
(W)\end{array}$ & $\begin{array}{c}\text { Fluorescence } \\
\text { Intensity } \\
(I)\end{array}$ & $\begin{array}{c}\text { Index of } \\
\text { Refraction } \\
(n)\end{array}$ & $\begin{array}{c}\text { Quantity } \\
\text { of Water } \\
(W)\end{array}$ & $\begin{array}{c}\text { Fluorescence } \\
\text { Intensity } \\
(I)\end{array}$ \\
\hline & $\begin{array}{c}\text { Sage- } \\
\text { Lavender, } \\
\text { Sample N7 }\end{array}$ & & & $\begin{array}{c}\text { Acacia, } \\
\text { Sample N4 }\end{array}$ & \\
\hline 1.4961 & 16.76 & 2.98 & 1.4976 & 16.16 & 1.45 \\
1.4835 & 21.80 & 2.22 & 1.4785 & 23.80 & 1.28 \\
1.4711 & 26.80 & 1.52 & 1.4651 & 29.20 & 0.96 \\
1.4651 & 29.20 & 1.34 & 1.4565 & 32.60 & 0.88 \\
1.4420 & 37.50 & 0.92 & 1.4282 & 44.00 & 0.70 \\
1.4222 & 46.40 & 0.78 & 1.4082 & 51.90 & 0,64 \\
1.4061 & 52.80 & 0.74 & 1.3871 & 60.40 & 0.62 \\
1.3849 & 61.20 & 0.66 & 1.3600 & 71.20 & 0.50 \\
1.3650 & 68.20 & 056 & 1.3500 & 75.20 & 0.32 \\
1.3489 & 75.60 & 0.42 & 1.3380 & 80.00 & 0.24 \\
1.3380 & 80.00 & 0.34 & - & - & - \\
\hline
\end{tabular}


100 Practical Spectroscopy in Agriculture and Food Science

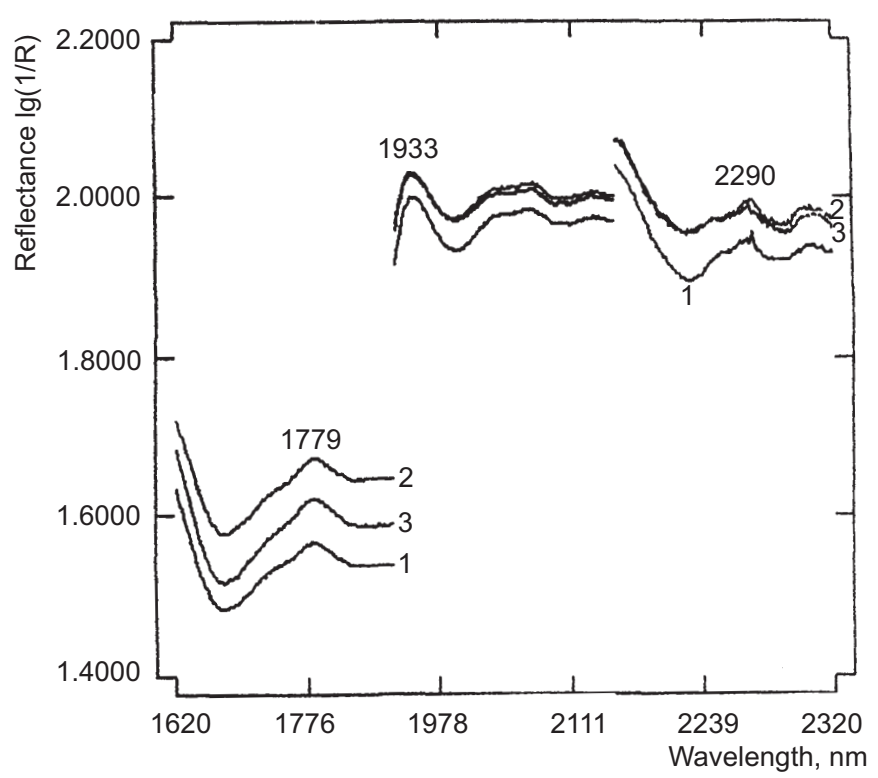

Fig. 6.4 Reflectance spectra of honey in near-infrared part of spectrum (samples N1-3) [Posudin et al., 1995]

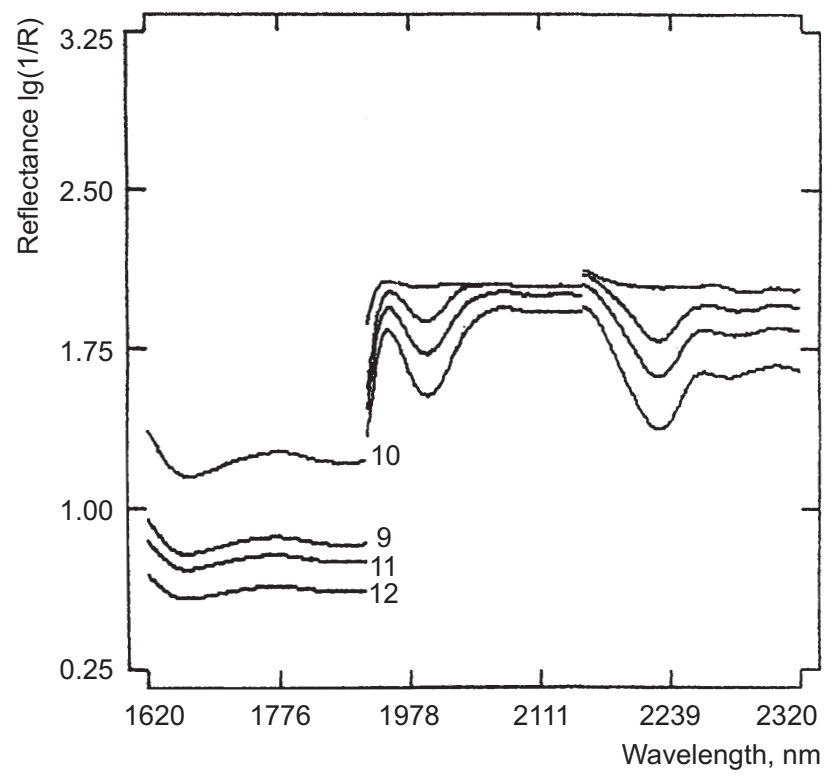

Fig. 6.5 Reflectance spectra of honey in near-infrared part of spectrum (samples N9-12) [Posudin et al., 1995] 


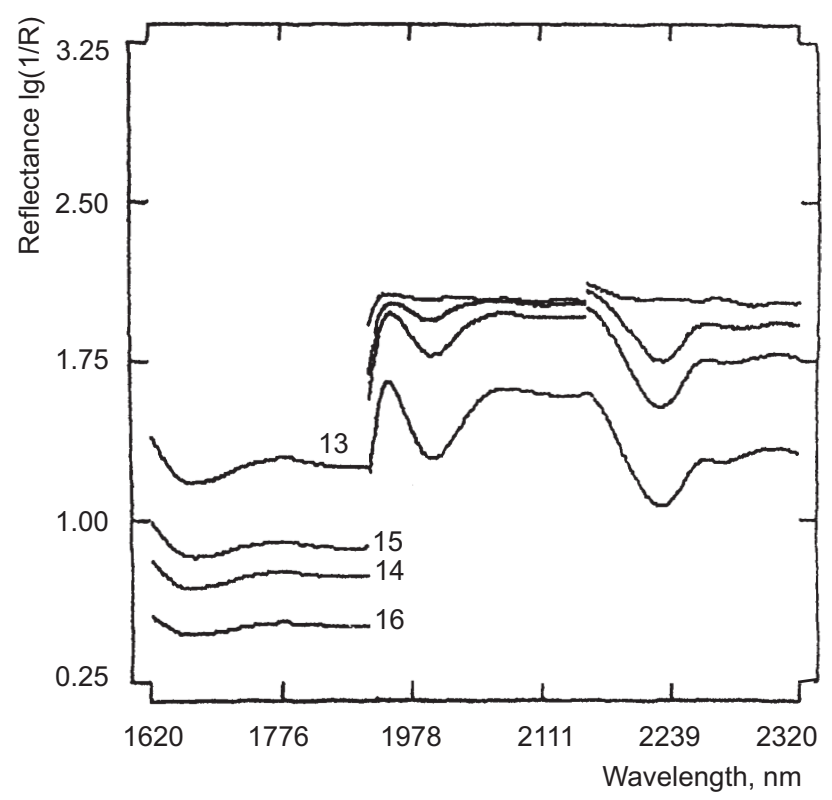

Fig. 6.6 Reflectance spectra of honey in near-infrared part of the spectrum (samples N1316) [Posudin et al., 1995]

It is very informative to use spectral parameters of honey for the nondestructive detection of honey adulteration. Figures 6.7 and 6.8 demonstrate the evolution of the reflectance spectra for the samples with different concentration of sucrose, which was artificially added to honey from lime-tree and multiherbal collection respectively. The curves "reflectance versus sucrose concentration" which are presented in Fig. 6.9, can be used for quantitative estimation of honey adulteration. The accuracy limits in these experiments did not exceed $5 \%$.

Thus, honey is characterized by certain spectral properties in ultraviolet, visible, and near-infrared parts of the spectrum, which are related to its ability to absorb, transmit, reflect and re-emit optical radiation. The chemical composition and physical properties of honey are closely related to its spectral parameters, which can be used as taxonomic indices or indicators of honey state and quality.

\subsection{SUMMARY}

Spectroscopic methods can be explored in honey-breeding. All the types of interaction of optical radiation with honey can be used in principle for fast and precise diagnostics of this product. The chemical composition, 
102 Practical Spectroscopy in Agriculture and Food Science

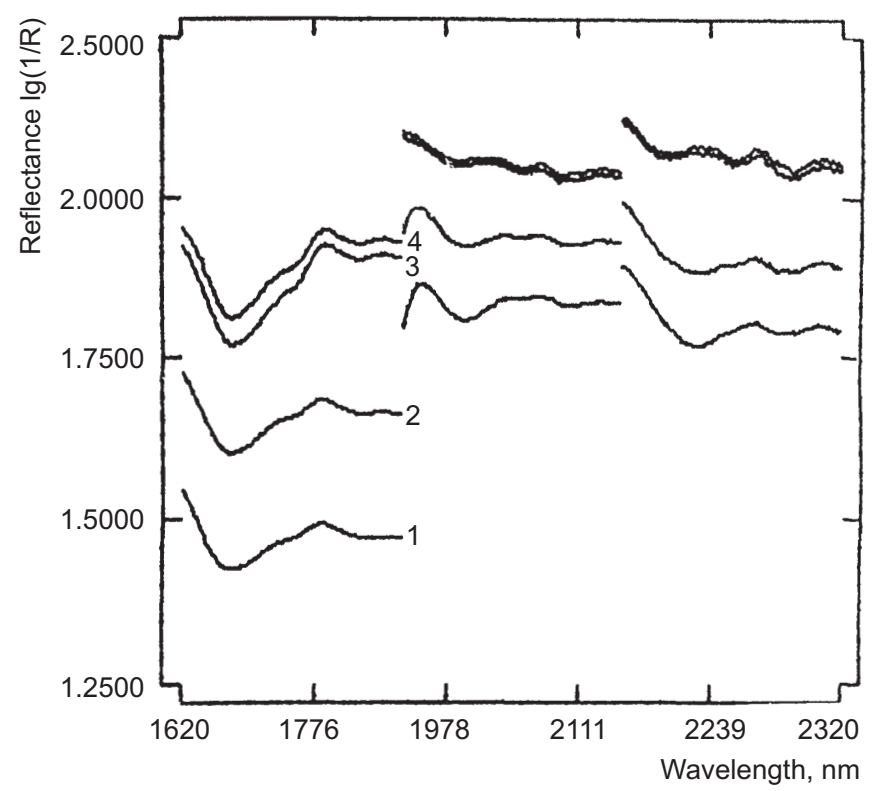

Fig. 6.7 Dependence of reflectance spectra of lime-tree honey (sample N17) in nearinfrared part of spectrum on the concentration of sucrose which was artificially added to honey. 1 - natural honey; $2-20 \%$ sucrose; $3-40 \%$ sucrose; $4-60 \%$ sucrose [Posudin et al., 1995]

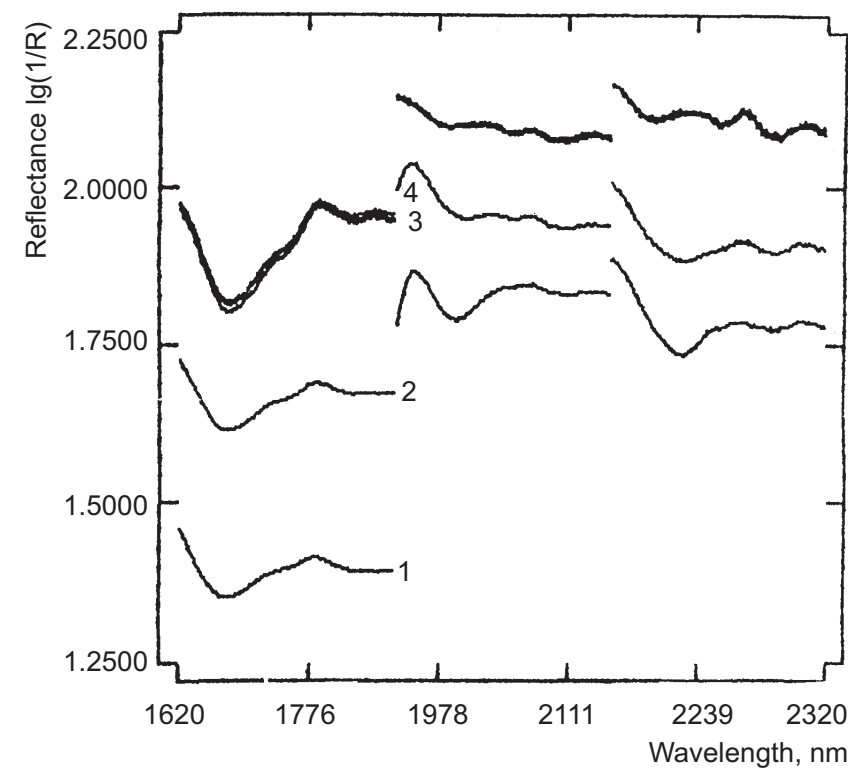

Fig. 6.8 Dependence of reflectance spectra of multiherbal honey in near-infrared part of spectrum on the concentration of sucrose which was artificially added to honey. 1 - natural honey; 2 - 20\% sucrose; $3-40 \%$ sucrose; $4-60 \%$ sucrose [Posudin et al., 1995] 
Spectroscopic Analysis of Honey $\mathbf{1 0 3}$

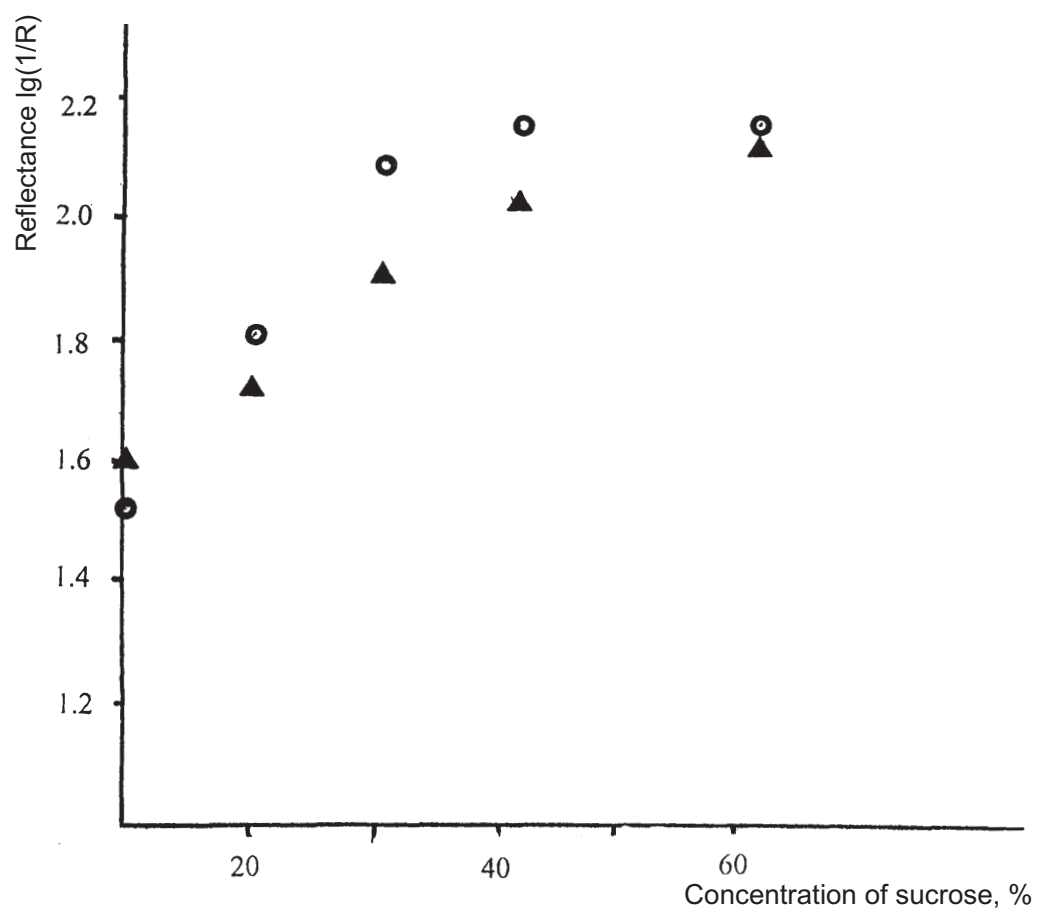

Fig. 6.9 Dependence of reflectance of honey on the concentration of sucrose. Wavelength is $1,779 \mathrm{~nm}$. - $\mathbf{\Delta -}-$ lime-tree honey, -0- - multiherbal honey [Posudin et al., 1995]

physical properties, geographic origin and age of honey are closely related to its spectral parameters which can be used as taxonomic indices or indicators of honey state and quality.

The near-infrared reflectance spectroscopy gives useful information about the adulteration of honey.

\subsection{REFERENCES}

Aganin, A.V. 1989. Sanitary Expertise of Honey. Saratov Agr. Inst. Publ., Saratov, Russia. p 40.

Campos, M.G. 1994. Controle de qualidade e tipificacao de meis, Apicultor. 2(6): 19-24.

Chudakov, V.G. 1967. Composition and Properties of Sugar Honey and Methodics of Detection of this Adulteration. Moscovsky Rabochy, Moscow, p 130.

Codex Alimentarius Comission CAS/RvS 12/1969, Recommended European Regional Standard for Honey, FAO/WHCXed., Rome. Codex Stan 12-1981, Rev.1 (1987), Rev.2 (2001).

Dustmann, J.H. 1993. Honey, quality and its control. American Bee Journal, 133(9): 648-651. 


\section{Practical Spectroscopy in Agriculture and Food Science}

Gonnet, M. 1986. L'analyse des miels. Description de queiques methodes de controle de la qualite, Bulletin-Technique-Apicole, 13(1): 17-36.

Jeanne, F. 1991. Le Miel. Definition, origines composition et proprietes.

Je'Anne, F. 1991. Le Miel. Definition, origines composition et proprietes, Buttetin-TechniqueApicole, 18(3), 76: 205-210.

Juan, T., Conchello, M.P., Tello, M.L., Perez-Arquille, C and Herrera, A. 1992. Rotation especifica y espcetro glucidico de mieles de Zaragoza, Attmentaria, 28(232): 75-78.

Mautz, D., Rosenkranz, P. and Schaper, F. 1993. Die Tätigkeit der B. Landesanstalt für Bienenzucht. Erlangen im Jahre 1991/92, Imkerfreund, 9: 1-29.

Petrovic, Z.T., Mandic, M.I., Grgic, J. and Grgic, Z. 1994. Ash and chromium levels of some types of honey, Zeitschrift für Lebensmittel-Untersuchung und Forschung, 198(1): 36-39.

Posudin, Yu.I. 1995. Biophysics. Urozhaj, Kyiv. p 222.

Posudin, Yu.I. 2005. Methods of Nondestructive Quality Evaluation of Agricultural and Food Products. Aristey, Kyiv. p 408.

Posudin, Yu.I., Polishuk V.P., Bulavin S.P. and Istomina, V.A. 1995. Spectroscopic methods of honey investigation. Visnyk agrarnoi nauki. 6: 78-82.

Salinas, F., Deespinosa, V.M., Osorio, E. and Lozano, M. 1994. Determination of mineral elements in honey from different floral origins by flow-injection analysis coupled to atomic spectroscopy, Revista Espanola De Ciencia Y Technologia De Ailmentos, 34(4): 441449.

Salinas, F., Espinosa-Mansilla, A. and Berzas-Nevado, J.J. 1994. Simultaneous determination of sulfathiazole and oxytetracycline in honey by derivative spectrophotometry, Microchemical Journal, 43(3): 244-252.

Vakhonina, T.V., Levina, L.P. and Bondareva, K.M. 1987. Quality of honey-pollen products, Pchelovodstvo. Moskva: Agropromizdat, 8: 28-29.

Vorwohl, G. 1990. Honigquatitatskontrolle und Honigqualitatskriterien in der Bundesrepublik, Bienenvater, 111(11): 391-398.

Vorwohl, G. 1984. Honey and other hive products, and their quality control, Proc. of the Third Intern. Conf. on Apiculture in Tropical Climates, Nairobi, Kenya, 5-9 Nov. 1984, p. $169-170$.

Yao, C. and Chen, L. 1985. Determination of traces of lead in honey by spectrophotometry with tetra(p-trimethylammoniumphenyl)porphine, Shipin-Yu-Fajiqo-Gongue, 6: 38-41.

Yao, C. and Fan, C. 1985. Determination of zinc in honey by spectrophotometry with 5, 10, 15, 20-tetrakis (4-trimethylammoniophenyl) porphine, Shipin-Yu-Fajiao-Gongue, 2: 1720. 


\section{Spectroscopic Analysis of Animal Hair and Bird Feathering}

\subsection{PROPERTIES OF ANIMAL HAIR-COVERING}

\subsubsection{Structure of Hair-covering}

A single hair consists of root with follicle, and hair shaft, which has components such as core, scab layer and cuticle (Fig. 7.1). The haircovering of the farm animals is characterized by the following parameters: the density of the hairs $\left(1 \mathrm{~cm}^{2}\right)$ is: 2,600 (cow), 3,000-4,000 (sheep), 700 (horse); the average thickness of a hair is (in $\mu \mathrm{m}$ ): $20-100$

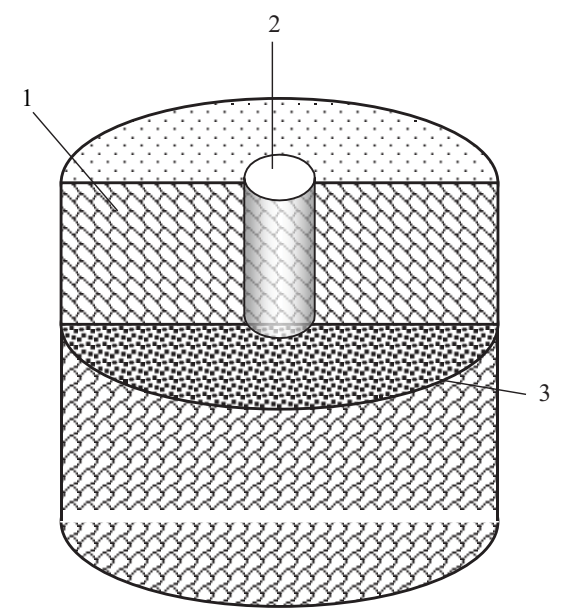

Fig. 7.1 A single hair consists of root with follicle, and hair shaft, which has components such as: 1 - scab layer, 2 - core, and 3 - cuticle 


\section{Practical Spectroscopy in Agriculture and Food Science}

(cow), 5-40 (sheep), 10-200 (horse). The density of hair is about $700 \mathrm{~cm}^{-2}$ for horse, $5,000-6,000 \mathrm{~cm}^{-2}$ for sheep.

\subsubsection{Colouration of Animal Hair-covering}

The colouration of animal hair-covering is determined by quantitative and qualitative characteristics of hair pigments. Melanin is the brown pigment which is produced by the specialized cells - melanocytes. These cells are located in the hair core. It is possible to distinguish three main groups of melanines - eumelanines, that are responsible for black and brown colour; pheomelanines, that produce yellow and red colour; trichochroms with low molecular weight. The dimensions of melanine granules in the hair are about $0.5-1.2 \mu \mathrm{m}$ for dark colour hair and 0.1-0.3 $\mu \mathrm{m}$ for white hair. The relative concentration of melanine depends on the colour of the hair cover and consists of 8.2-8.4 for dark and about 1.7 for light colour (\% from mass of the sample).

The structure, composition and properties of pigments depend on the phase of hair growth [Seiji, 1973], content or deficiency of metals [Kostaneski, 1970], presence of vitamins $A$ and $D$ in feeding [Wojcikowska-Soroczynska et al., 1987], solar radiance [Guidance to study skin-covering of mammals, 1988], and violation of blood vessel system [Durst, 1963].

As a whole, colour of the hair can be considered as an important genotypical sign [Ioganson et al., 1970].

\subsection{HAIR SAMPLES}

The first group of the samples was presented by the hair-covering of the horses from sportive club of National Agricultural University. Different types of colouration such as black, bay, grey and chestnut were chosen. The samples were taken from the head, side and mane of the horse [Posudin et al., 1993].

The second group of the samples were taken from the hair-covering of ponies. The samples were chosen from the head, neck, side, croup, extremities and nails of the horses [Posudin et al., 1993].

The third group of the samples used were from the hair-covering of the Przevalsky horse (Equus przewalskii) taken from the collection of Ukrainian Research Institute of the Cattle-Breeding of Steppe Regions "Askaniya-Nova". The samples were taken from the cheek, neck, shoulder blade, back, side, stomach, croup, extremities, mane, and tail (Fig. 7.2) of 22 different horses [Posudin et al., 1998]. Two main colour types of the Przevalsky horse within the same herd are known [Poliakov, 


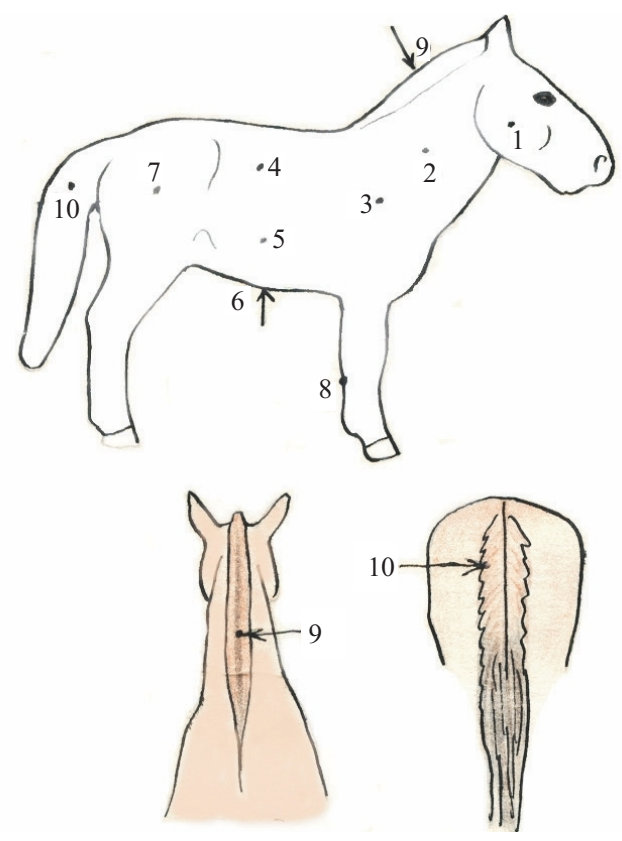

Fig. 7.2 Samples taken from 22 different horses. 1 - cheek, 2 - neck, 3 - shoulderblade, 4 - back, 5 - side, 6 - stomach, 7 - croup, 8 - extremities, 9 - mane, 10 - tail [Posudin et al., 1998]

1881; Bannikov, 1958; Groves, 1986; Klimov, 1990]: one pale grey-yellow, the other a bright yellowish red-brown. There are intermediate types of colouration also. A dark mono-coloured type can be distinguished which has less marked contrast between the dark head neck and the body; this type has dark muzzle and black legs (Fig. 7.3); a greyish type with the head and neck that are darker than the body; this type has a white muzzle, grey hindlegs and dark grey forelegs, and several thin dark zebra-like stripes on the carpus (Fig. 7.4); the others are light types, either normal with a white muzzle, zebra-like stripes on the carpus, light grey forelegs and light hindlegs (Fig. 7.5), or mono-coloured with a white muzzle, light forelegs and very light hindlegs (Fig. 7.6).

The fourth group of the animal hair that was used in the experiments with laser diffractometry consisted of the vibrissa of 371 heads of bull calves and heifers which were compared with the mass of the animals [Posudin and Trofimenko, 1994]. 
108 Practical Spectroscopy in Agriculture and Food Science

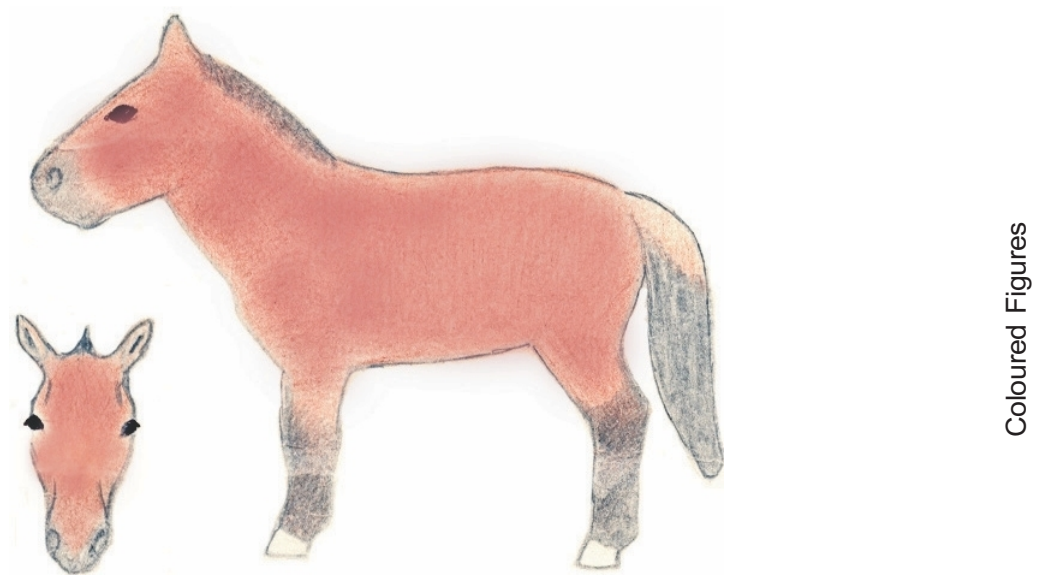

Fig. 7.3 A dark mono-coloured type which has less marked contrast between the dark head, neck and the body; this type has a dark muzzle and black legs [Posudin et al., 1998]

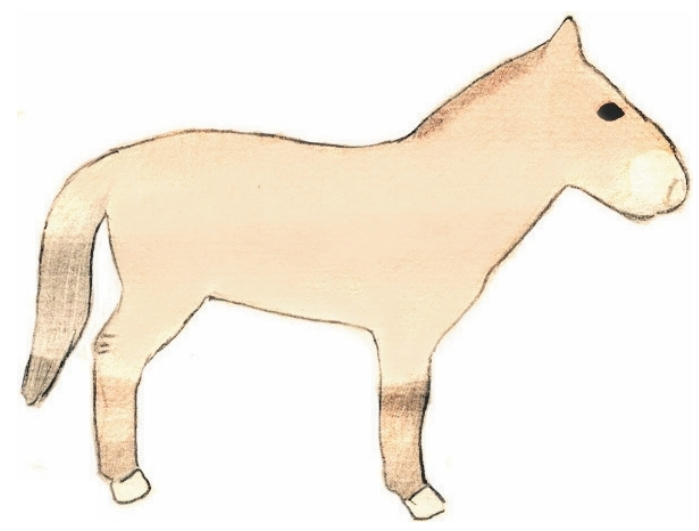

Fig. 7.4 A greyish type with the head and neck that are darker than the body; this type has a white muzzle, grey hindlegs and dark grey forelegs, and several thin dark zebra-like stripes on the carpus [Posudin et al., 1998]

\subsection{INSTRUMENTATION}

\subsubsection{Spectrometer}

The spectrometer which was used for microphotometry and microfluorometry of animal hair consisted of incandescent lamp OI-9 1, lens 2, sample 3, microscope LUMAM-3M 4, photomultiplier FEU-79 5, recorder H-306 6, digital voltmeter V7-21 7, mercury lamp DRSh-250 8, 


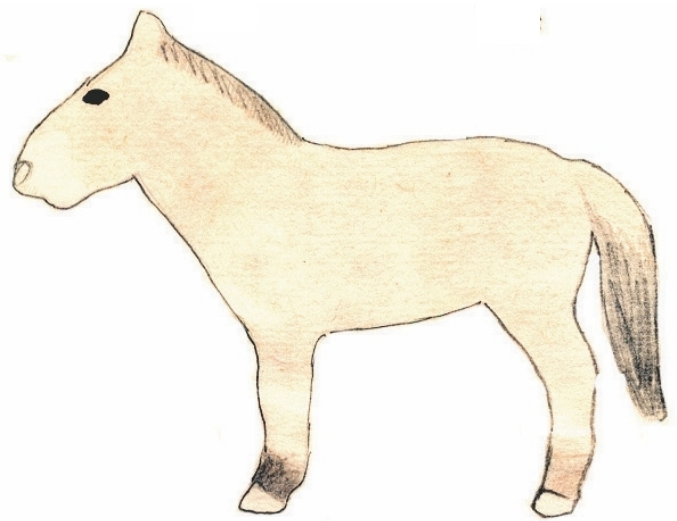

Fig. 7.5 A light normal type with a white muzzle, zebra-like stripes on the carpus, light grey forelegs and light hindlegs [Posudin et al., 1998]

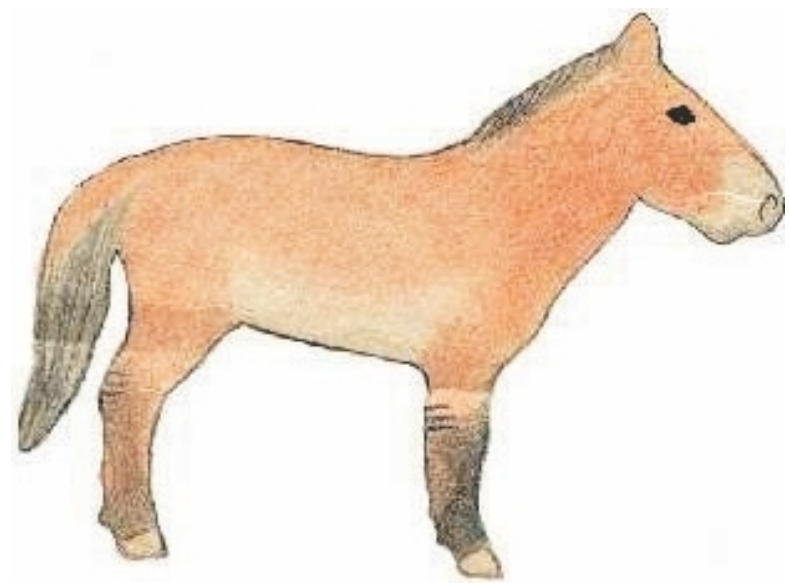

Fig. 7.6 A mono-coloured light type with a white muzzle, light forelegs and very light hindlegs [Posudin et al., 1998]

glass infrared filter 9, water infrared filter 10, halogen lamp KGM9-70 11, monochromator UM-2 12, and semitransparent plate 13 (Fig. 7.7).

The procedure of microphotometry included the passage of light from the incandescent lamp through the hair and microscope and subsequent registration of the transmitted light by the photomultiplier. A signal $S=$ $\left(I-I_{N}\right) /\left(I_{0}-I_{N}\right)$ was registered during microphotometry of the hair, where $I$ and $I_{0}$ are signals which were recorded with and without the hair correspondingly, and $I_{N}$ was the level of noise which was estimated with the closed photomultiplier. 


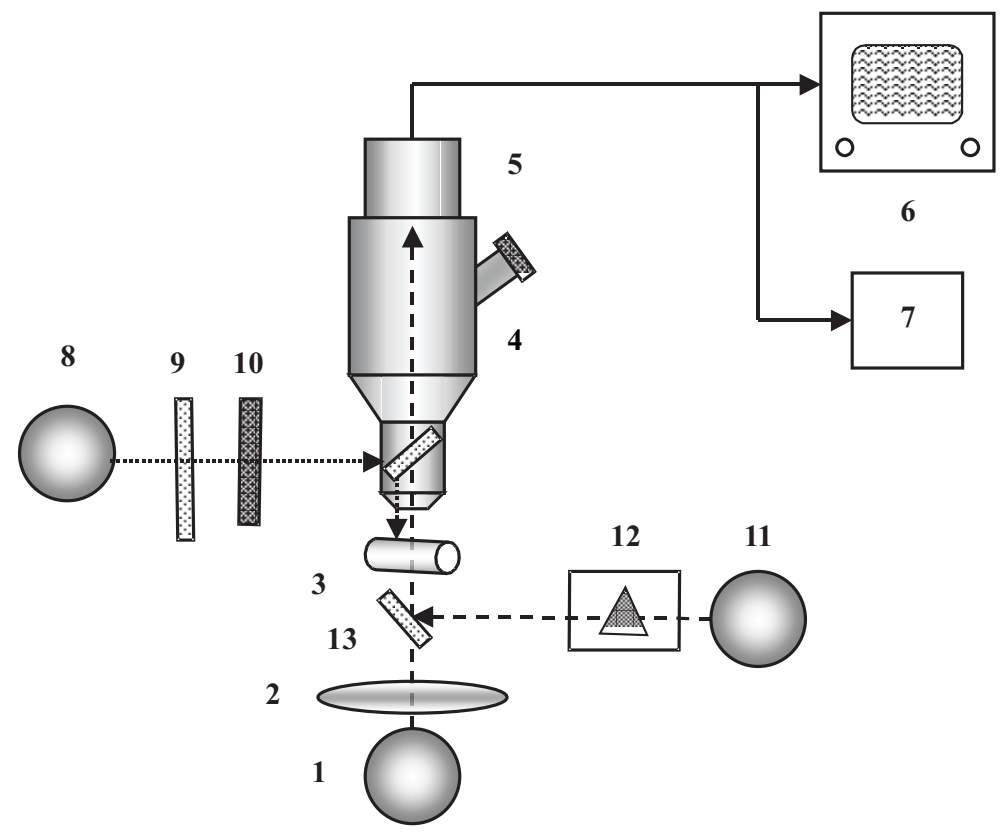

Fig. 7.7 The spectrometer which was used for microphotometry and microfluorometry of animal hair: 1 - incandescent lamp OI-9; 2 - lens; 3 - sample; 4 - microscope LUMAM3M; 5 - photomultiplier FEU-79; 6 - recorder H-306; 7 - digital voltmeter V7-21; 8 mercury lamp DRSh-250; 9 - glass infrared filter; 10 - water infrared filter; 11 - halogen lamp KGM9-70; 12 - monochromator UM-2; 13 - semitransparent plate [Posudin et al., 1991]

Microfluorometry of hair was based on the excitation of fluorescence of the sample by radiation of the mercury lamp which passed through filters on the sample. Fluorescence emission radiation was registered by the photomultiplier and readout system.

The measurement of the illuminance of the sample during the experiments was realized by luxmeter Yu-116.

\subsubsection{Laser Diffractometry}

Let us consider the situation when laser radiation is made to pass through a single slit. The author and his colleagues used He:Ne-laser LGN-215 (wavelength $632 \mathrm{~nm}$ ) in their experiments. Some of the radiation appears in the geometric shadow of the aperture. It is shown [Born and Wolf, 1970] that the intensity of monochromatic light diffracted by a slit is related to the width $d$ of the slit, the angle of diffraction $\theta$ and the wavelength $\lambda$ of light as follows: 


$$
I=I_{0} \sin \alpha / \alpha^{2}
$$

where $I_{0}$ is the intensity of the incident light and $\alpha=d \sin \theta / \lambda$.

It is possible to show with certain accuracy that the intensity of light diffracted by a thin cylinder (animal hair or vibrissa) of the same size $d$ can be described by the following equation:

$$
I=I_{0}(1-\sin \alpha / \alpha)^{2}
$$

While measuring the interval between diffraction maxima of a certain order of diffraction, it is possible to estimate the diameter $d$ of the slit or cylinder (the thickness of the hair). This is the main idea of laser diffractometry. This interval $l_{m}$ between diffractional maxima and distance $L$ between the sample and the screen where diffraction is observed are related to the expression:

$$
\sin \theta_{m}=\frac{l_{m}}{\sqrt{l_{m}^{2}+L^{2}}}
$$

where $\theta_{m}$ is the angle of registering the diffraction maximum of the $m$-th order (Fig. 7.8).

As soon as the condition of observation of diffraction maxima can be written as:

$$
\alpha_{m}=\frac{4 m-1}{2} \pi=\frac{\pi d \sin \theta_{m}}{\lambda}
$$

it is possible to obtain the expression which connects the diameter $d$ of the hair with parameters $l_{m}$ and $L$ :

$$
\alpha_{m}=\frac{4 m-1}{2} \lambda \sqrt{\frac{l_{m}^{2}+L^{2}}{l_{m}}}
$$

\subsection{RESULTS OF SPECTROSCOPIC ANALYSIS OF ANIMAL HAIR}

\subsubsection{Microphotometry and Microspectrophotometry of Horse Hair}

The colour of horses is a rather important biological sign; each horse race has a dominating colour. It is known [Combs, 1987] that the colour of the horse hair is a specific indicator of the accumulation of calcium and lead. The colour of the hair also determines the ability of the horse to resist the 
112 Practical Spectroscopy in Agriculture and Food Science

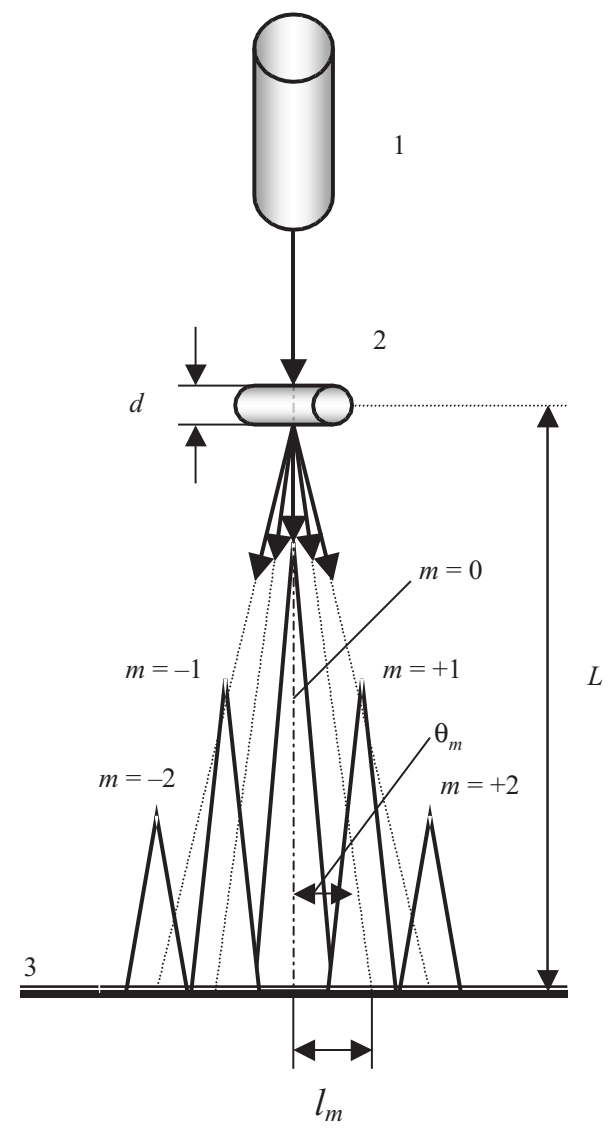

Fig. 7.8 Principle of laser diffractometry: $I_{m}-$ interval between diffractional maxima; $L-$ distance between the sample and the screen where diffraction is observed; $\theta_{m}$ - the angle of registering the diffraction maximum of the $m$-th order [Posudin et al., 1991]

photosensitizing action of fagopyrin which is found in Fagopyrum (Polygonum or F. esculentum) during solar irradiation. For example, grey horses suffer more form fagopyrism which leads to melanosarcoma [Gopka et al., 1989]. In addition, the colour of horse hair is related to the relative ratio of melanine and 3,4-dioxyphenylalanine [Durst, 1936].

The dependence of transmittance $\tau$ of horse hair on the illuminance $E$ is presented in Figs. 7.9-7.12. It is clear that the curves $\tau=f(E)$ depend strongly on the colour and the part of animal body. Each curve is characterized by a linear part of dependence $\tau=f(E)$ and the region of saturation, where $\tau \rightarrow 1$. The hair of grey colouration has maximal 
Spectroscopic Analysis of Animal Hair and Bird Feathering $\mathbf{1 1 3}$

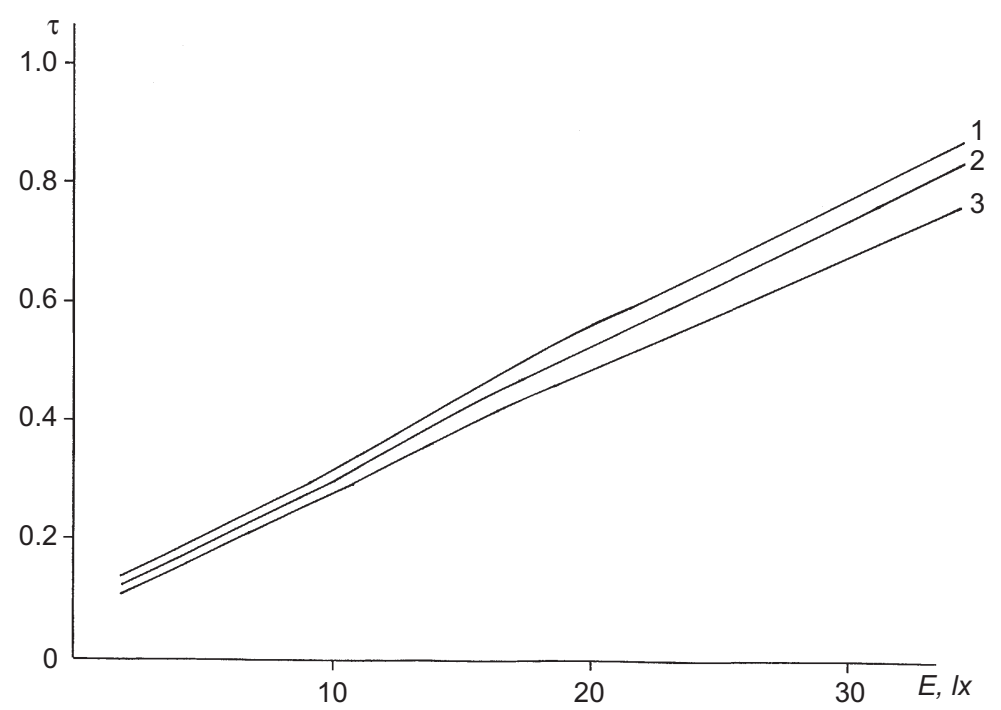

Fig. 7.9 Dependence of transmittance $\tau$ of black horse hair on the illuminance $E$. 1 - side; 2 - mane; 3 - head [Posudin et al., 1991]

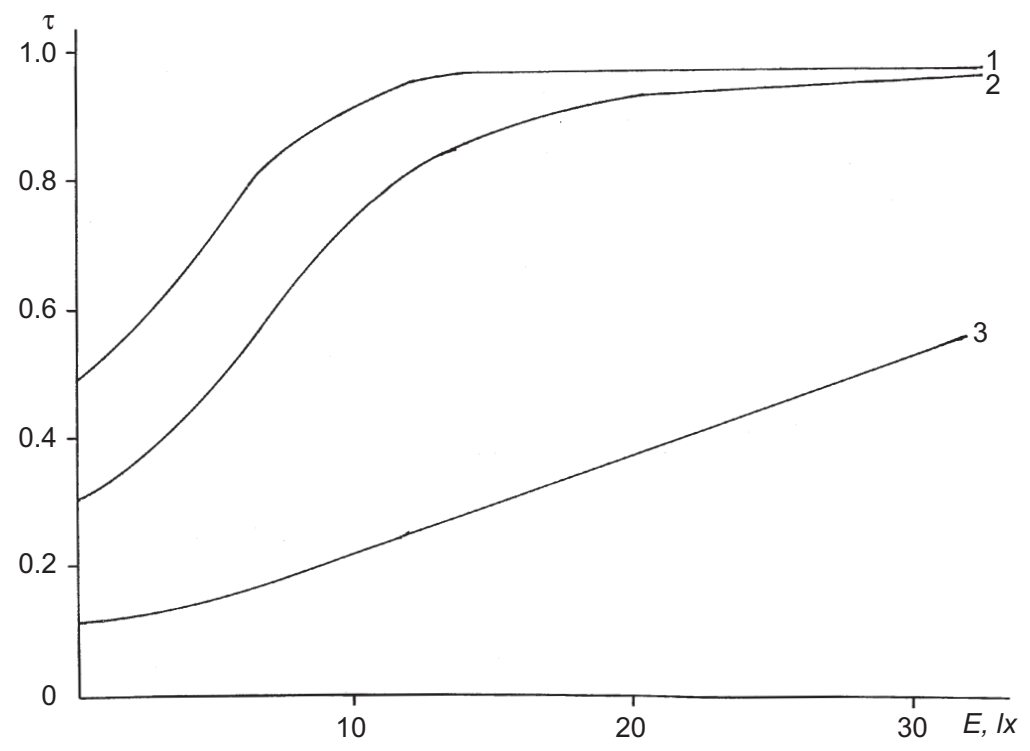

Fig. 7.10 Dependence of transmittance $\tau$ of bay horse hair on the illuminance $E$. 1 - head; 2 - side, 3 - mane [Posudin et al., 1991] 
114 Practical Spectroscopy in Agriculture and Food Science

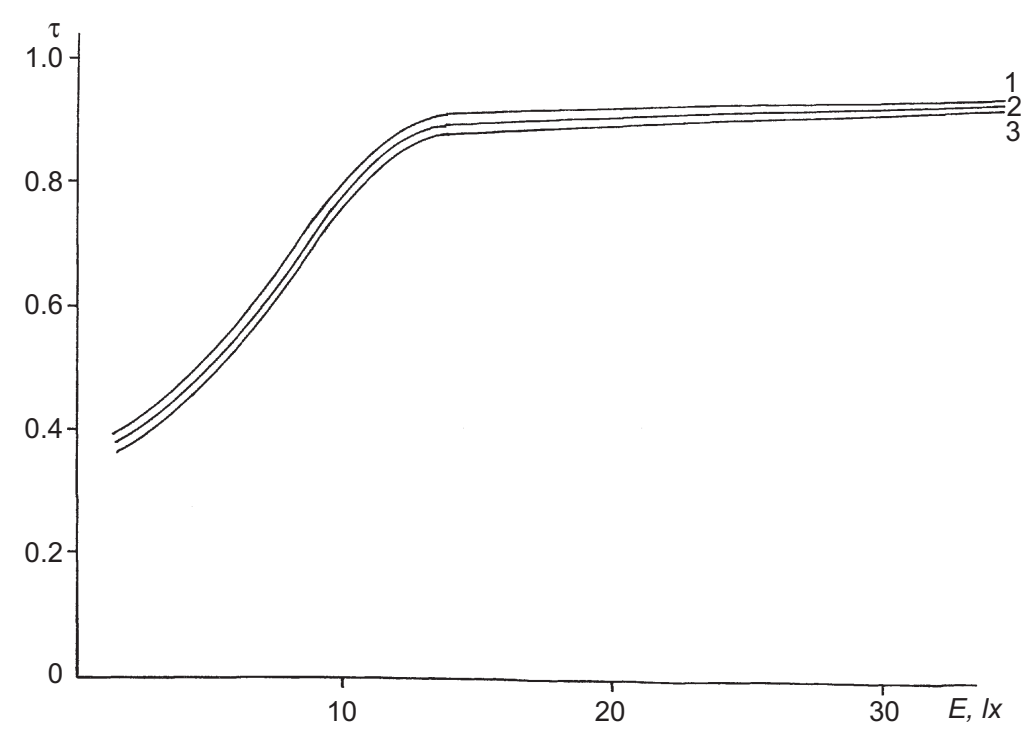

Fig. 7.11 Dependence of transmittance $\tau$ of chestnut horse hair on the illuminance $E$. 1 - head; 2 - side; 3 - mane [Posudin et al., 1991]

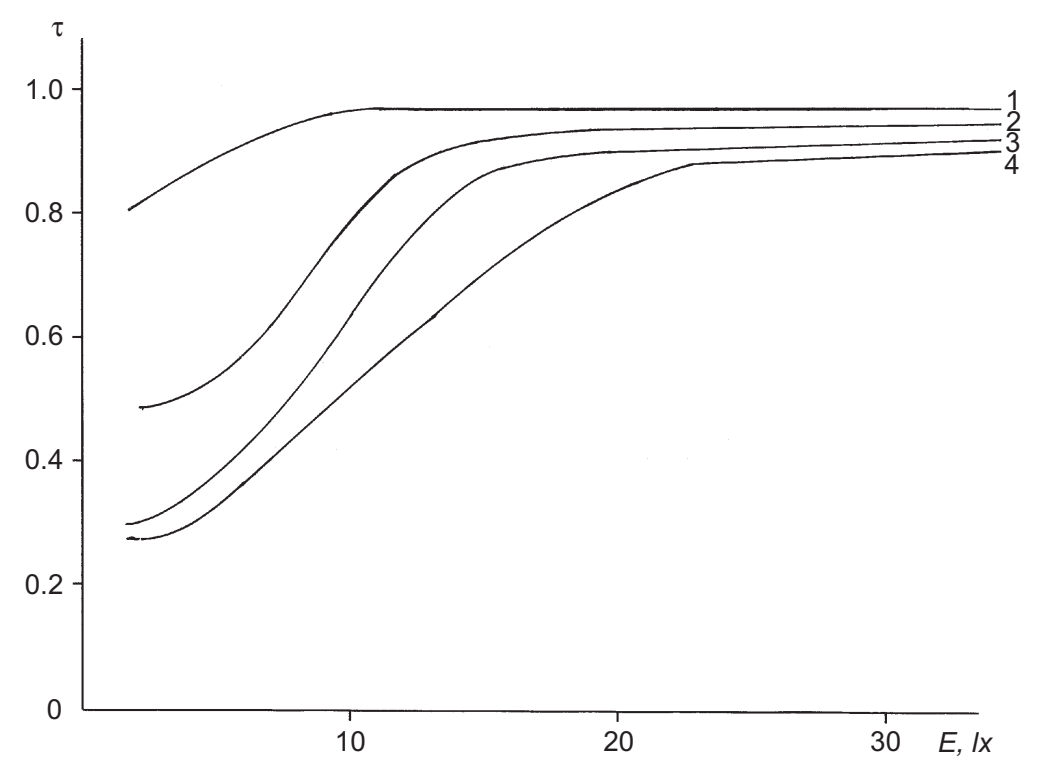

Fig. 7.12 Dependence of transmittance $\tau$ of grey horse hair on the illuminance $E$. 1 - mane (core), 2 - mane (crust), 3 - head, 4 - side [Posudin et al., 1991] 
transmission, while minimal transmission is typical for the hair of black colouration. The high values of light transmission by grey hair can be explained by early age grizzling, stopping formation of pigments and decreasing of the alkalinity of blood [Durst, 1936]. The linear dependence $\tau=f(E)$ can be used for quantitative estimation of colour of hair-covering of horses.

The typical spectra of transmission of the horse hair are presented in Figs. 7.13-7.16. The character of these spectra depends on the colour, part of the hair (peripherical or central) and the region of animal body. The microphotometry of the hair through the core demonstrates maximal transmission in long-wavelength region for black, chestnut and grey colour. The hair of each colour has different half-widths of transmission bands (black and chestnut colours have maximal half-widths). Transmission of light in the short-wavelength region makes up about 30$40 \%$. The character of transmission spectrum of horse hair depends on the pigment content, the process of light refraction inside the hair, illuminance conditions and distribution of air in internal cavities of the hair [Durst, 1936]. The transmission spectra which were registered by the author can be used for quantitative and qualitative estimation of pigment content in the hair.

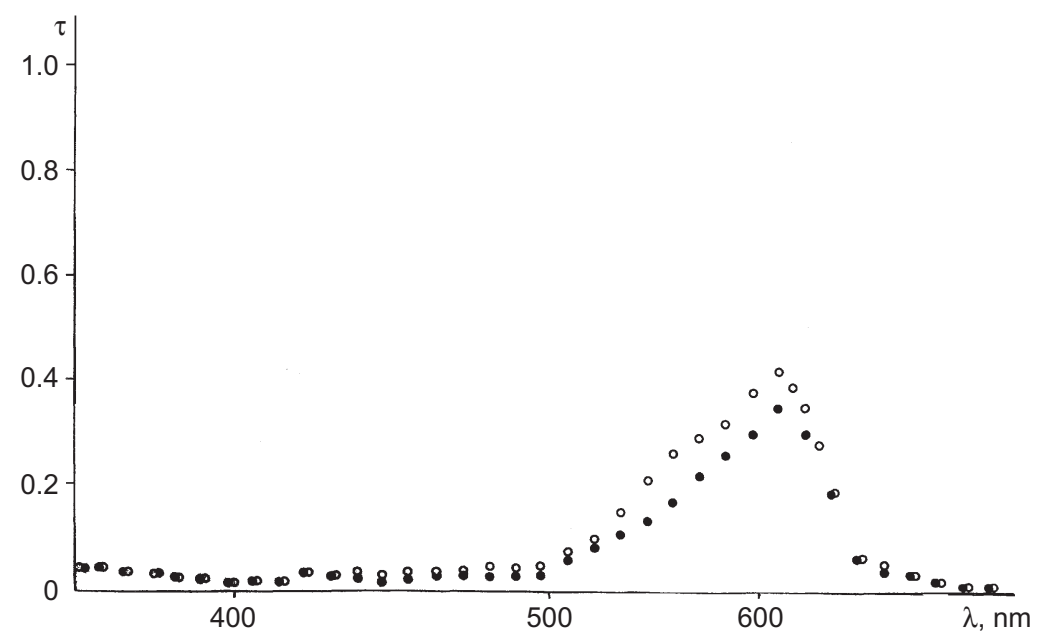

Fig. 7.13 Transmittance spectra of the hair for black colour horse: - o - - head (core); - - - - head (crust) [Posudin et al., 1991] 
116 Practical Spectroscopy in Agriculture and Food Science

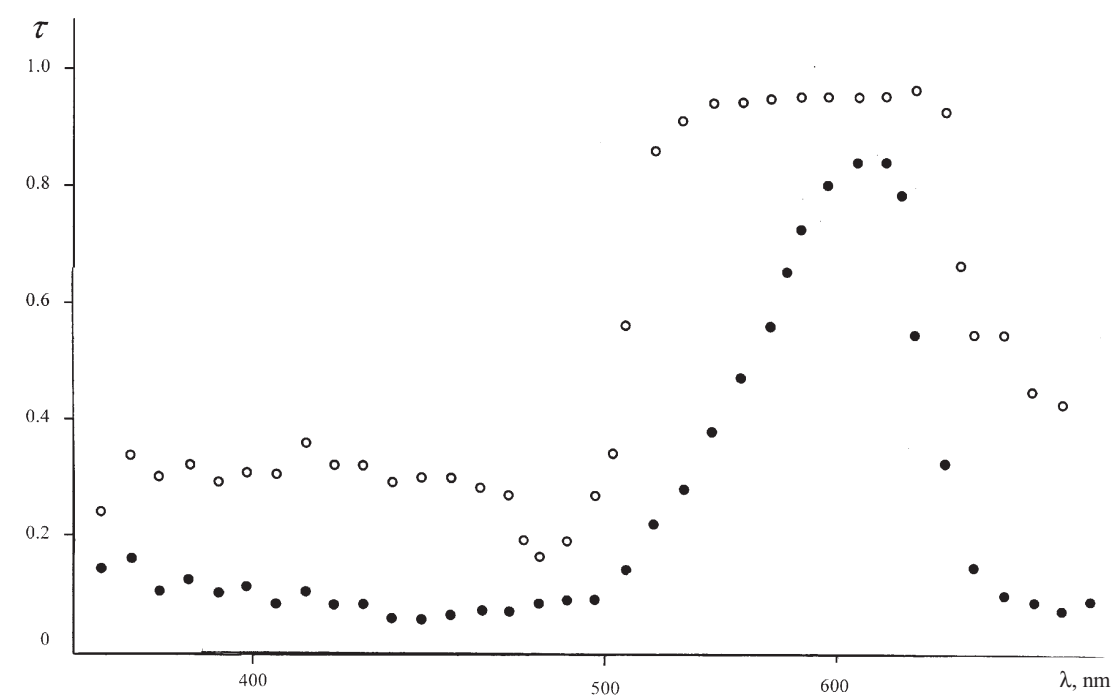

Fig. 7.14 Transmittance spectra of the hair for chestnut colour horse: - o - - head (core); - - - head (crust) [Posudin et al., 1991]

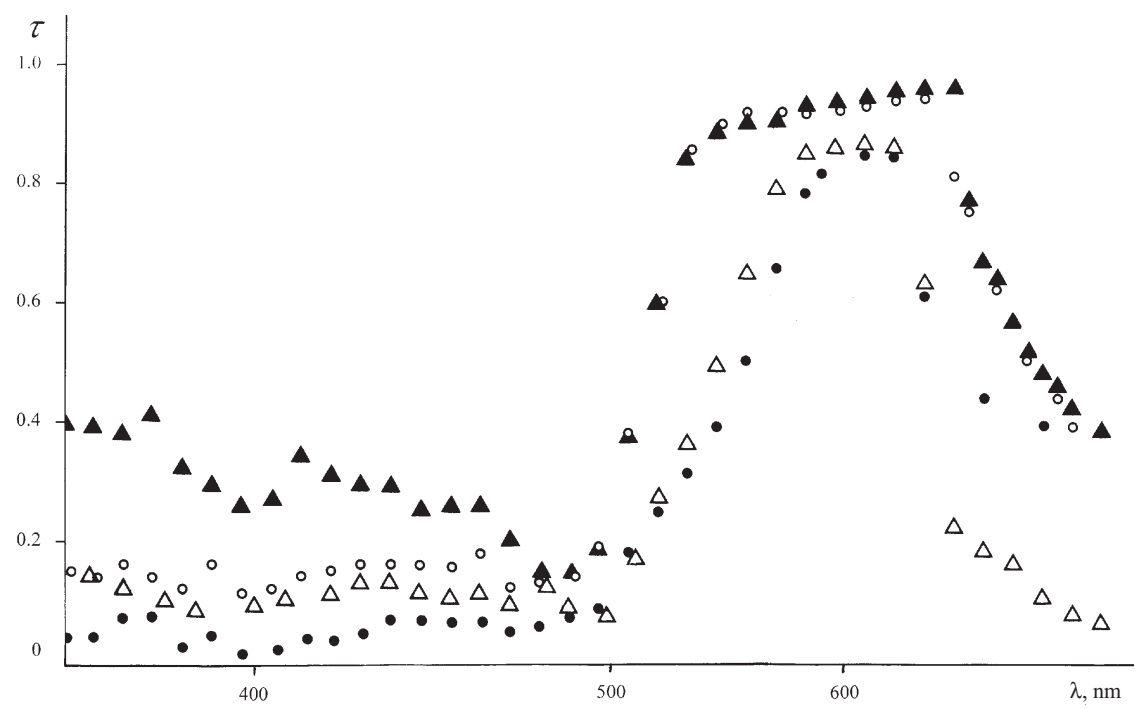

Fig. 7.15 Transmittance spectra of the hair for bay colour horse: - $\Delta--$ head (crust); - $\Delta$ - - head (core); - o - - neck (core); - • - - neck (crust) [Posudin et al., 1991] 


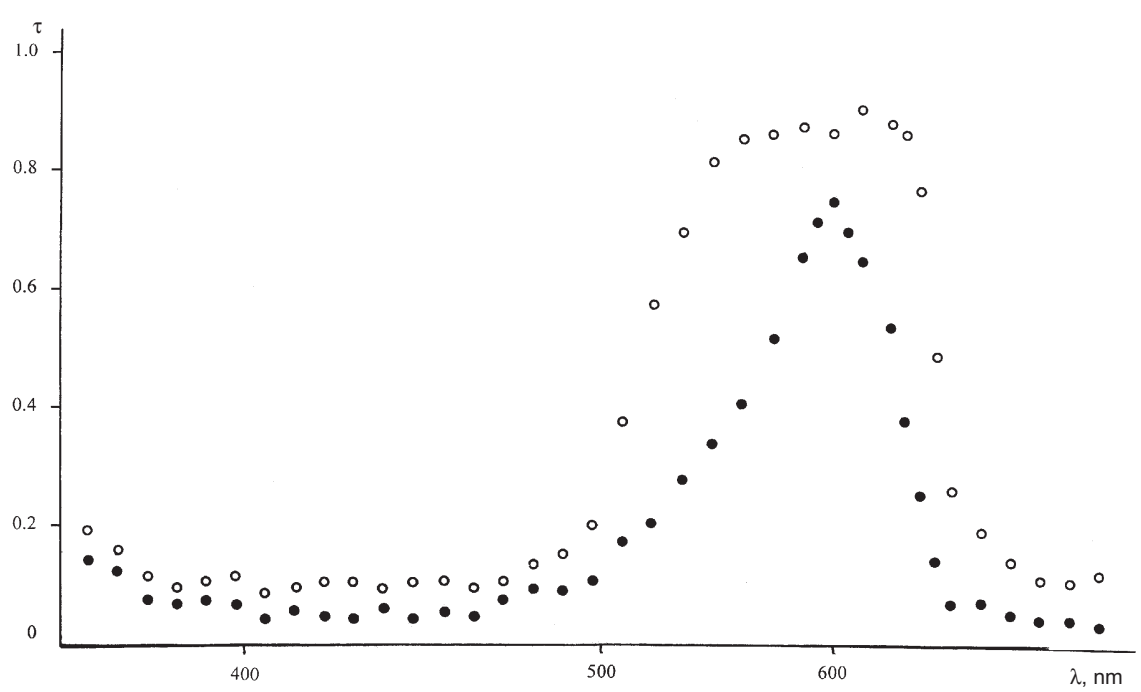

Fig. 7.16 Transmittance spectra of the hair for grey colour horse: - o - - head (core); - - - head (crust) [Posudin et al., 1991]

\subsubsection{Microfluorometry of Horse Hair}

Effect of parts of the body. The method of microfluorometry was applied to the quantitative estimation of pigmentation of the hair-covering of sportive horses, ponies and the Przevalsky horse [Posudin et al., 1991; 1993; 1998].

Dependence of the fluorescence intensity of the hair on the part and colour of the sportive horse body is presented in Fig. 7.17. The maximal intensity is observed for the protective hair of light grey horses. If the colour of grey hair can be taken into account as $100 \%$, the colour of the integumentary hair makes up $60 \%$ for light grey colour, $30 \%$ for dark grey colour, $13-18 \%$ for black colour, $25-30 \%$ for bay colour and $27-37 \%$ for chestnut colour.

Dependence of the fluorescence intensity of the hair on the part of pony body (stallion of black colouration, mare of chestnut colouration and their foal) is presented in Fig. 7.18.

The intensity of fluorescence of the hair samples of the Przevalsky horse depends on the part of horse body (22 individuals have been investigated). Such parts as back, side, croup and shoulder blade demonstrate maximal values of fluorescence, while tail, extremities and mane - minimal values (Table 7.1, Fig. 7.19). 
118 Practical Spectroscopy in Agriculture and Food Science

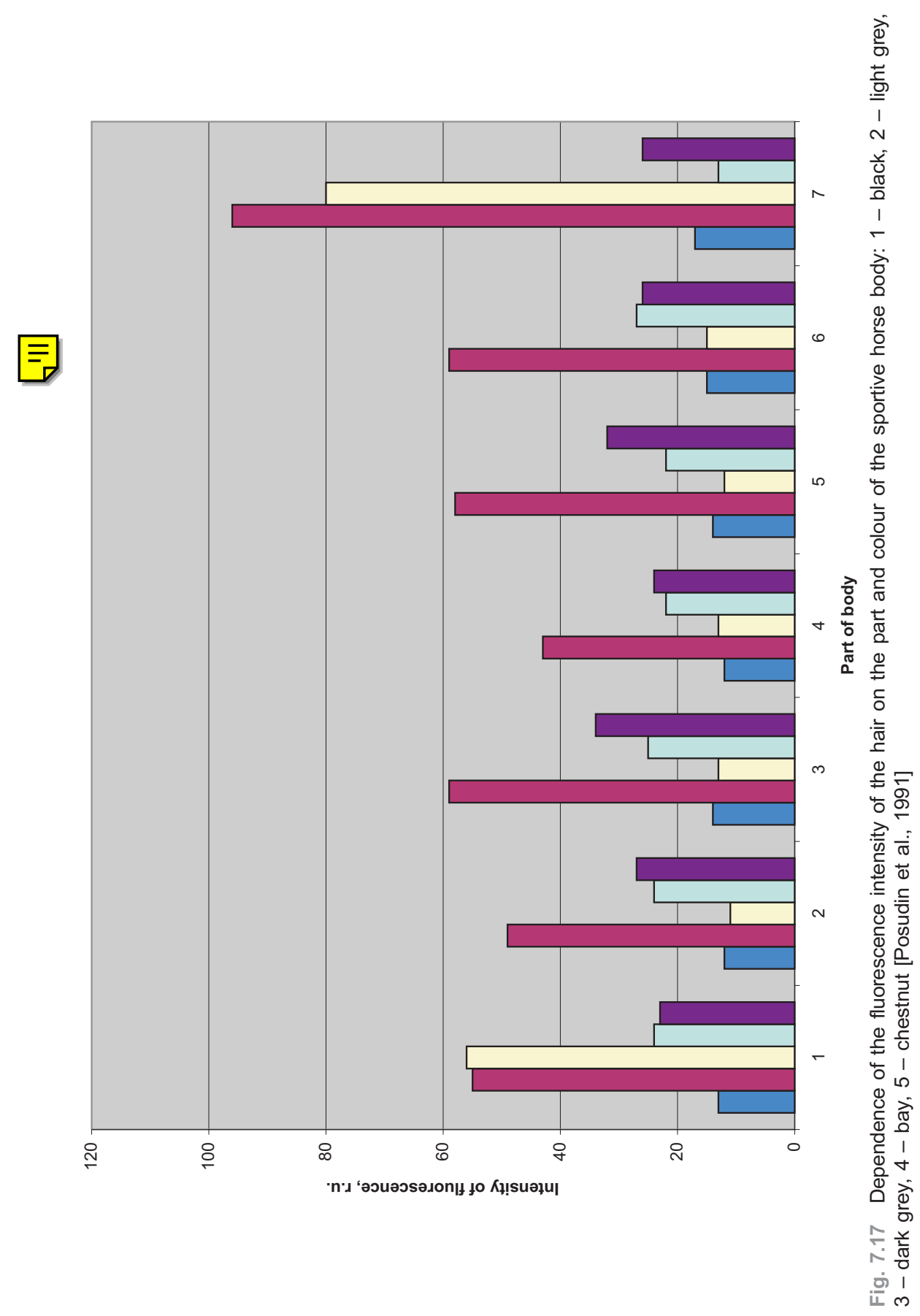


Spectroscopic Analysis of Animal Hair and Bird Feathering 119

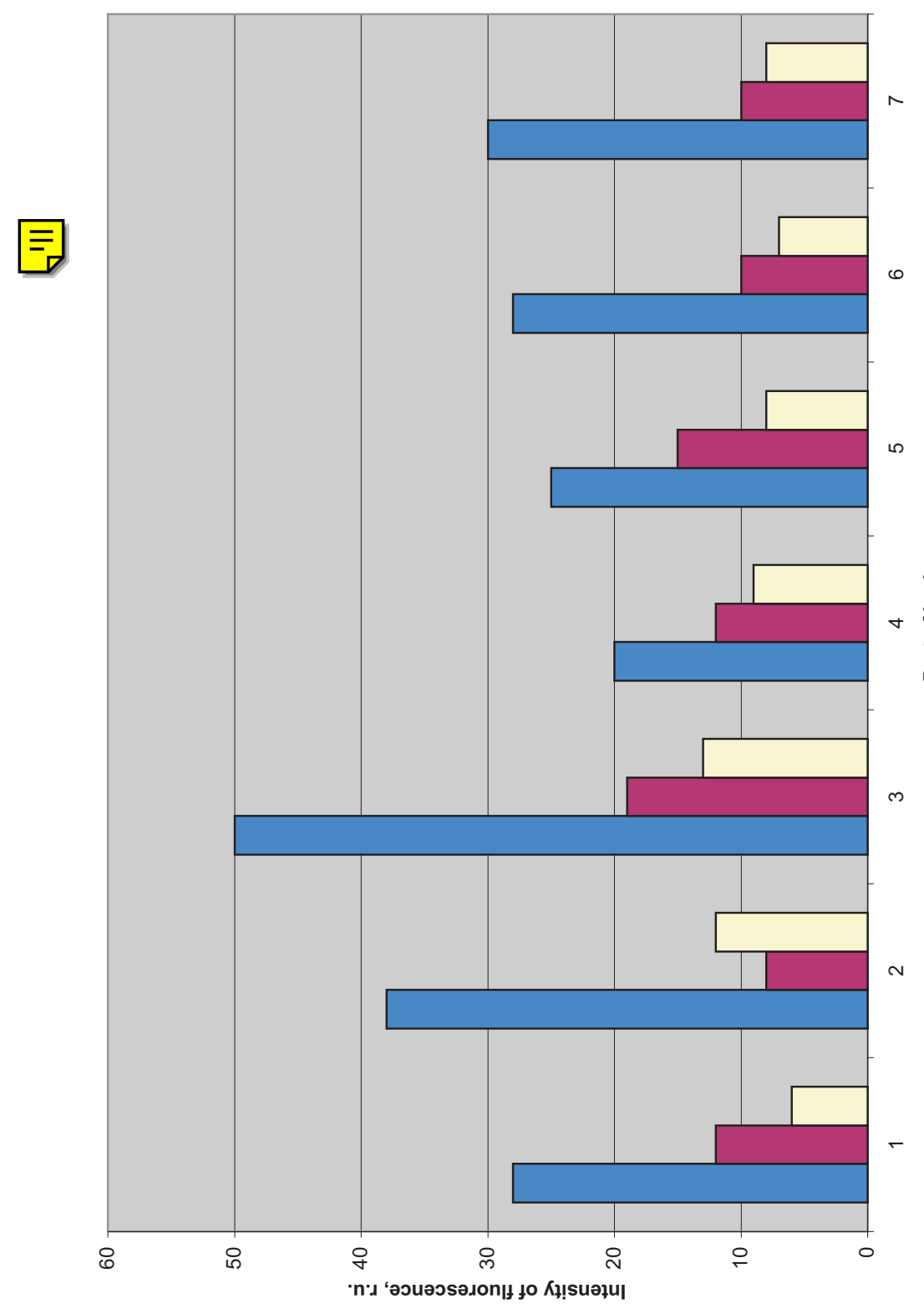

$\overrightarrow{5}$
$\frac{5}{0}$
$\frac{0}{0}$

पั

$\stackrel{\frac{0}{\sigma}}{\frac{1}{\varepsilon}}$

ธิ

을

$0 \quad \frac{1}{0}$

4

음

1) ㅎํㅇ

टे

구을

$+\frac{2}{0} \frac{0}{\sigma}$

贾

ธั ธ

늘 कू

는

ডัర

त

Ф

ป ํㅡㄹ

¿

D $N$

¿

을 음

은 혼

$\stackrel{\oplus}{\risingdotseq}$ !

吹

ㅎํㄴ

웡 뭉

ธ

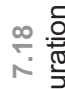

훈응 
120 Practical Spectroscopy in Agriculture and Food Science

Table 7.1 Dependence of the fluorescence intensity of hair-covering of the Przevalsky horse on parts of the body

\begin{tabular}{l|c|c}
\hline Part of Body & Number of Samples & Intensity of Fluorescence \\
\hline Cheek & 63 & $44.09 \pm 4.85$ \\
Neck & 68 & $49.84 \pm 4.53$ \\
Shoulder blade & 69 & $55.36 \pm 4.11$ \\
Back & 72 & $61.19 \pm 5.13$ \\
Side & 72 & $58.03 \pm 5.28$ \\
Stomach & 66 & $49.18 \pm 5.29$ \\
Croup & 72 & $57.29 \pm 4.88$ \\
Extremities & 15 & $23.76 \pm 2.38$ \\
Mane & 54 & $16.07 \pm 1.97$ \\
Tail & 57 & $22.11 \pm 2.71$ \\
\hline
\end{tabular}

These results correlate first of all with colouration of the Przevalsky horse: in spite of diversity of types and varieties, this horse shows definite tendencies in colour of hair-covering (Table 7.2).

Table 7.2 Coefficient of correlation of fluorescence intensity between different parts of the body of Przevalsky horse

\begin{tabular}{l|c|c|c}
\hline Part of Body & Coefficient of Correlation & $t_{d}$ & $P$ \\
\hline Cheek-back & 0.590 & 4.300 & $>0.999$ \\
Cheek-back & 0.370 & 2.210 & $>0.950$ \\
Cheek-croup & 0.390 & 2.560 & $>0.950$ \\
Cheek-back & 0.400 & 2.660 & $>0.950$ \\
Neck-croup & 0.530 & 3.990 & $>0.999$ \\
Neck-back & 0.460 & 3.150 & $>0.900$ \\
Shoulder blade-croup & 0.530 & 5.990 & $>0.999$ \\
Shoulder blade-stomach & 0.350 & 1.990 & $>0.950$ \\
Shoulder blade-mane & 0.400 & 2.300 & $>0.950$ \\
Back-croup & 0.680 & 6.080 & $>0.999$ \\
Back-side & 0.430 & 3.140 & $>0.900$ \\
Back-stomach & 0.440 & 3.020 & $>0.900$ \\
Back-tail & 0.350 & 2.870 & $>0.950$ \\
Side-stomach & 0.570 & 4.440 & $>0.999$ \\
Side-croup & 0.410 & 3.080 & $>0.900$ \\
Side-mane & 0.470 & 3.390 & $>0.900$ \\
Side-tail & 0.380 & 2.700 & $>0.950$ \\
Stomach-extremities & 0.610 & 4.030 & $>0.999$ \\
Stomach-tail & 0.780 & 7.890 & $>0.999$ \\
Stomach-croup & 0.410 & 2.860 & $>0.900$ \\
Stomach-mane & 0.440 & 2.960 & $>0.900$ \\
Croup-mane & 0.440 & 3.060 & $>0.900$ \\
Croup-extremities & 0.380 & 2.310 & $>0.950$ \\
Extremities-tail & 0.460 & 2.920 & $>0.900$ \\
\hline
\end{tabular}


Spectroscopic Analysis of Animal Hair and Bird Feathering $\mathbf{1 2 1}$

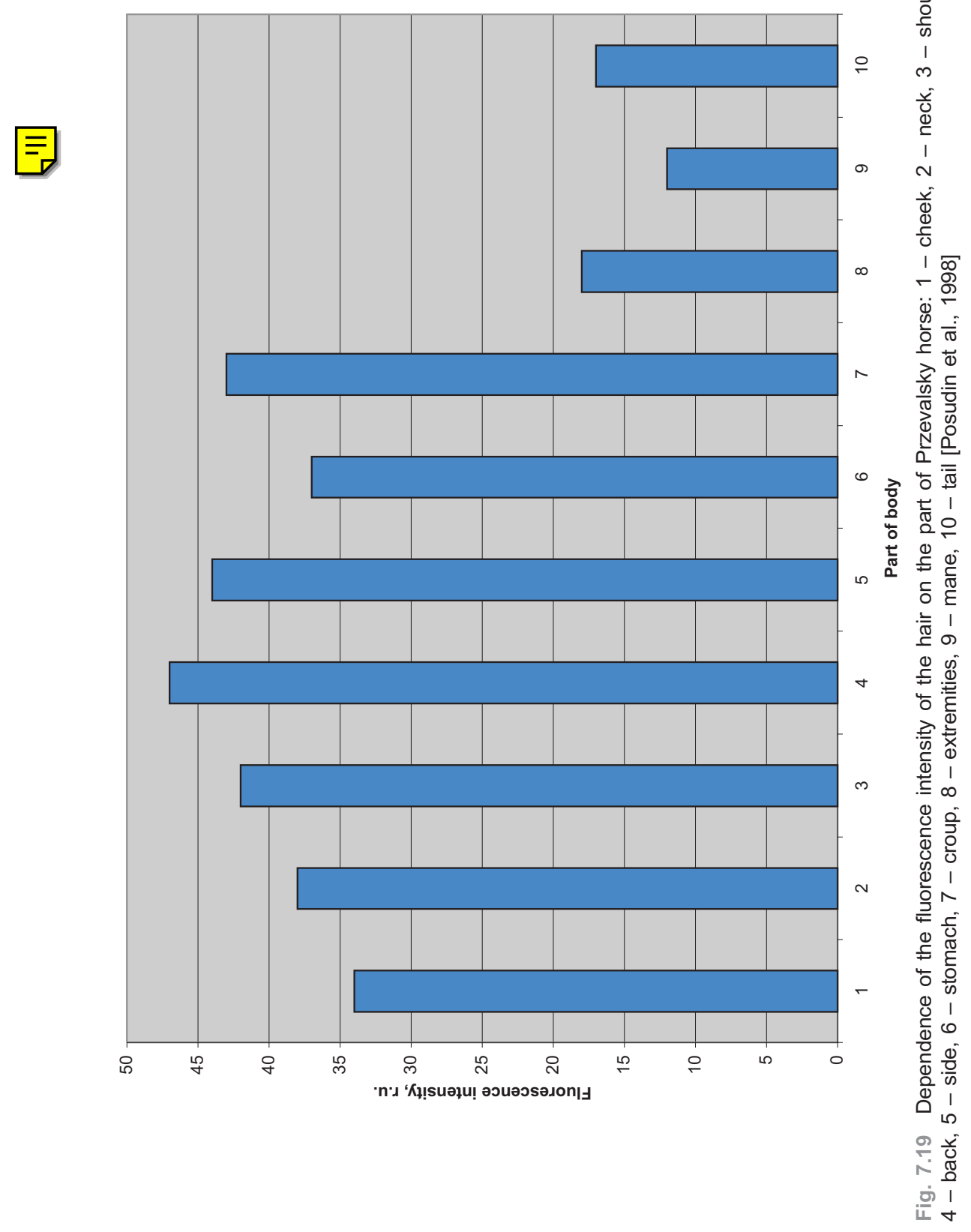




\section{Practical Spectroscopy in Agriculture and Food Science}

The intensity of fluorescence is related to the colouration of haircovering, for example, stallion "Vidny" demonstrates intensity of fluorescence $17 \pm 1$ for black hair, $143 \pm 2$ for white hair, $252 \pm 10$ for chestnut hair; stallion "Polkan" $31 \pm 7$ for black hair and $127 \pm 9$ for white one. The correlation between intensity of fluorescence and colouration of the hair can be explained by different quantity of hair melanine - the hair of dark colouration absorbs light more intensively and re-emits light more weakly.

We did not establish the dependence of hair fluorescence on the sex of the animal, for example, the intensity of fluorescence of the hair from the side was $58.86 \pm 6.69$ for mare (number of samples 42 ) and 58.86 \pm 8.68 for stallion (number of samples 30 ); the intensity of fluorescence of the hair from neck was $58.62 \pm 6.33$ for mare (number of samples 39 ) and $38.01 \pm 35.33$ for stallion (number of samples 29) the difference was not statistically significant.

The results of the experiments demonstrated the dependence of fluorescence intensity of the hair-covering on the season - the value of this parameter increased in summer from $5.33 \pm 0.33$ to $11.67 \pm 0.88$ $\left(t_{d}=6.81 ; P>0.99\right)$ for neck hair, from $9.67 \pm 2.19$ to $20.67 \pm 6.67\left(t_{d}=7.5\right.$; $P>0.99)$ for hair on the back, from $2.00 \pm 0.00$ to $2.67 \pm 0.39\left(t_{d}=2.03\right.$; $P>0.9)$ for mane hair, and decreased from $25.00 \pm 0.33$ to $16.33 \pm 1.53$ $\left(t_{d}=3.57 ; P>0.95\right)$ for hair on the side and from $22.00 \pm 1.25$ to $5.67 \pm 0.67$ $\left(t_{d}=11.5 ; P>0.999\right)$ for side hair. The difference between the intensity of fluorescence for other parts of the body was not statistically significant.

It was interesting to study the dependence of fluorescence parameters on the age of the animal. We have studied the age change of fluorescence of the hair of mare "Vorkuta" at the period of time 17 days, 4 months 22 days, and one year 4 months. The hair of the young horse (17 days) demonstrates the maximal values of the intensity of fluorescence (Table 7.3).

Table 7.3 Age changes of fluorescence of hair of Przevalsky horse

\begin{tabular}{l|c|c|c|c|c}
\hline \multirow{2}{*}{ Age } & $\begin{array}{c}\text { Date of } \\
\text { Experiment }\end{array}$ & \multicolumn{4}{|c}{$\begin{array}{c}\text { Intensity of Fluorescence of the Hair } \\
\text { from Shoulder Blade, r.u. }\end{array}$} \\
\cline { 3 - 6 } & & $n$ & $l \pm \sigma^{2}$ & $t_{d}$ & $P$ \\
\hline 17 Days & 01.06 .1993 & 6 & $20.00 \pm 2.73$ & 0.03 & $<0.95$ \\
4 Months 23 days & 14.10 .1993 & 6 & $11.17 \pm 1.01$ & 1.52 & $>0.90$ \\
1 Year 3 months & 01.09 .1994 & 6 & $11.67 \pm 0.80$ & 3.49 & $>0.95$ \\
\hline
\end{tabular}


Determination of genotypic indices. The main objective of these experiments was possible application of fluorescence parameters of animal (pony and Przevalsky horse) hair as genotypic signs of animal diversity. The author's and his colleague's attention was drawn to the hair of Scotch pony-stallion "Medok" of black colouration, Estonian pony-mare "Damy" of chestnut colouration and their foal of one year old [Posudin et al., 1993]. The investigation of fluorescence parameters of horse hair as genotypic signs was continued with the hair-covering of the Przevalsky horse. The hair samples were taken from stallion "Vizor 313", mare "Volga 244", and foal "Vetka 490" [Posudin et al., 1998].

The spectral characteristics (intensity of fluorescence) of the hair samples which were taken from different parts of the body of the pony were investigated. The following ratio was used for determination of predomination of genetic levels of the posterity [Plokhinsky, 1964]:

$$
F=\frac{S+k M}{1+k}
$$

where $k$ is the coefficient which is determined experimentally as:

$$
\begin{gathered}
k=\frac{S-M+2 \Delta}{S-M-2 \Delta}=\frac{M-F}{F-S} \\
D=F-\frac{M+S}{2}
\end{gathered}
$$

Here symbols $S$ and $M$ correspond to the fluorescence intensity of the samples which were taken from hair-covering of stallion and mare correspondingly; $k$ is a level of increasing of genetic influence of parents; $\Delta$ is exceeding of mean level of the descendant sign over half-sum of mean levels of parents.

The results of measurement of fluorescence intensity of hair which were taken from different parts of the body are presented in Table 7.4 (pony) and Table 7.5 (Przevalsky horse) correspondingly.

It is shown that fluorescence intensity of the foal hair takes the intermediate place between the values of the fluorescence intensity of the parent's hair for pony (Fig. 7.18) and for the Przevalsky horse (Fig. 7.20).

Application of the expression (Eqn. 7.6) made it possible to elucidate which parent - stallion or mare - provides dominating genetic information. The results of calculation are presented in Table 7.6 for pony and in Table 7.7 for the Przevalsky horse. 
124 Practical Spectroscopy in Agriculture and Food Science

Table 7.4 Dependence of fluorescence intensity of pony hair on parts of the pony body

\begin{tabular}{|c|c|c|c|}
\hline \multirow{3}{*}{ Part of Body } & \multicolumn{3}{|c|}{$I \pm \sigma^{2}$} \\
\hline & \multicolumn{2}{|c|}{ Parents } & \multirow[b]{2}{*}{ Foal } \\
\hline & Stallion & Mare & \\
\hline Head & $30.67 \pm 2.90$ & $5.67 \pm 1.20$ & $8.33 \pm 4.70$ \\
\hline Neck & $22.67 \pm 3.10$ & $10.00 \pm 0.80$ & $13.00 \pm 4.10$ \\
\hline Side & $27.00 \pm 4.30$ & $9.33 \pm 1.90$ & $17.00 \pm 3.50$ \\
\hline Croup & $30.33 \pm 6.90$ & $7.00 \pm 0.80$ & $11.67 \pm 0.90$ \\
\hline Extremities & $32.00 \pm 2.40$ & $9.33 \pm 1.20$ & $10.67 \pm 0.90$ \\
\hline Tail & $56.67 \pm 3.40$ & $15.00 \pm 1.00$ & $21.00 \pm 1.60$ \\
\hline
\end{tabular}

Table 7.5 Dependence of fluorescence intensity of pony hair on parts of the Przevalsky horse body

\begin{tabular}{l|r|r|r}
\hline \multirow{2}{*}{ Part of Body } & \multicolumn{2}{|c}{$I \pm \sigma^{2}$} \\
\cline { 2 - 3 } & \multicolumn{2}{|c}{ Parents } \\
\cline { 2 - 4 } & \multicolumn{1}{|c}{ Stallion } & $164.00 \pm 1.90$ & Foal \\
\hline Cheek & $48.00 \pm 2.80$ & $170.00 \pm 0.90$ & $126.00 \pm 4.90$ \\
Back & $80.00 \pm 3.90$ & $155.00 \pm 2.60$ & $128.00 \pm 3.80$ \\
Side & $66.00 \pm 4.20$ & $62.00 \pm 2.80$ & $121.00 \pm 5.90$ \\
Croup & $103.00 \pm 6.90$ & $103.00 \pm 1.20$ & $99.00 \pm 3.00$ \\
Stomach & $84.00 \pm 3.50$ & $20.00 \pm 1.00$ & $8.00 \pm 0.90$ \\
Mane & $17.00 \pm 2.50$ & $32.00 \pm 4.30$ & $25.00 \pm 2.70$ \\
Tail & $24.00 \pm 2.80$ &
\end{tabular}

Table 7.6 Effect of non-additive domination on genotype signs for pony family

\begin{tabular}{l|c|c|c|c|c}
\hline $\begin{array}{c}\text { Part of } \\
\text { Body }\end{array}$ & $k$ & $\Delta$ & $\begin{array}{c}\text { Formation of Genetic } \\
\text { Level of Descendant }\end{array}$ & $\begin{array}{c}\text { Coefficient } \\
\text { of Regression } \\
\text { for Mare }\end{array}$ & $\begin{array}{c}\text { Coefficient of } \\
\text { Regression } \\
\text { for Stallion }\end{array}$ \\
\hline Head & 0.12 & -9.84 & $F=(M+0.12 S) / 1.12$ & 0.89 & 0.11 \\
Neck & 0.31 & -3.33 & $F=(M+0.31 S) / 1.31$ & 0.76 & 0.24 \\
Side & 0.77 & -1.17 & $F=(M+0.77 S) / 1.77$ & 0.56 & 0.44 \\
Croup & 0.25 & -7.00 & $F=(M+0.25 S) / 1.25$ & 0.80 & 0.20 \\
Extremities & 0.06 & -10.00 & $F=(M+0.06 S) / 1.06$ & 0.94 & 0.06 \\
Tail & 0.17 & -14.84 & $F=(M+0.17 S) / 1.17$ & 0.85 & 0.15 \\
\hline
\end{tabular}


Spectroscopic Analysis of Animal Hair and Bird Feathering $\mathbf{1 2 5}$

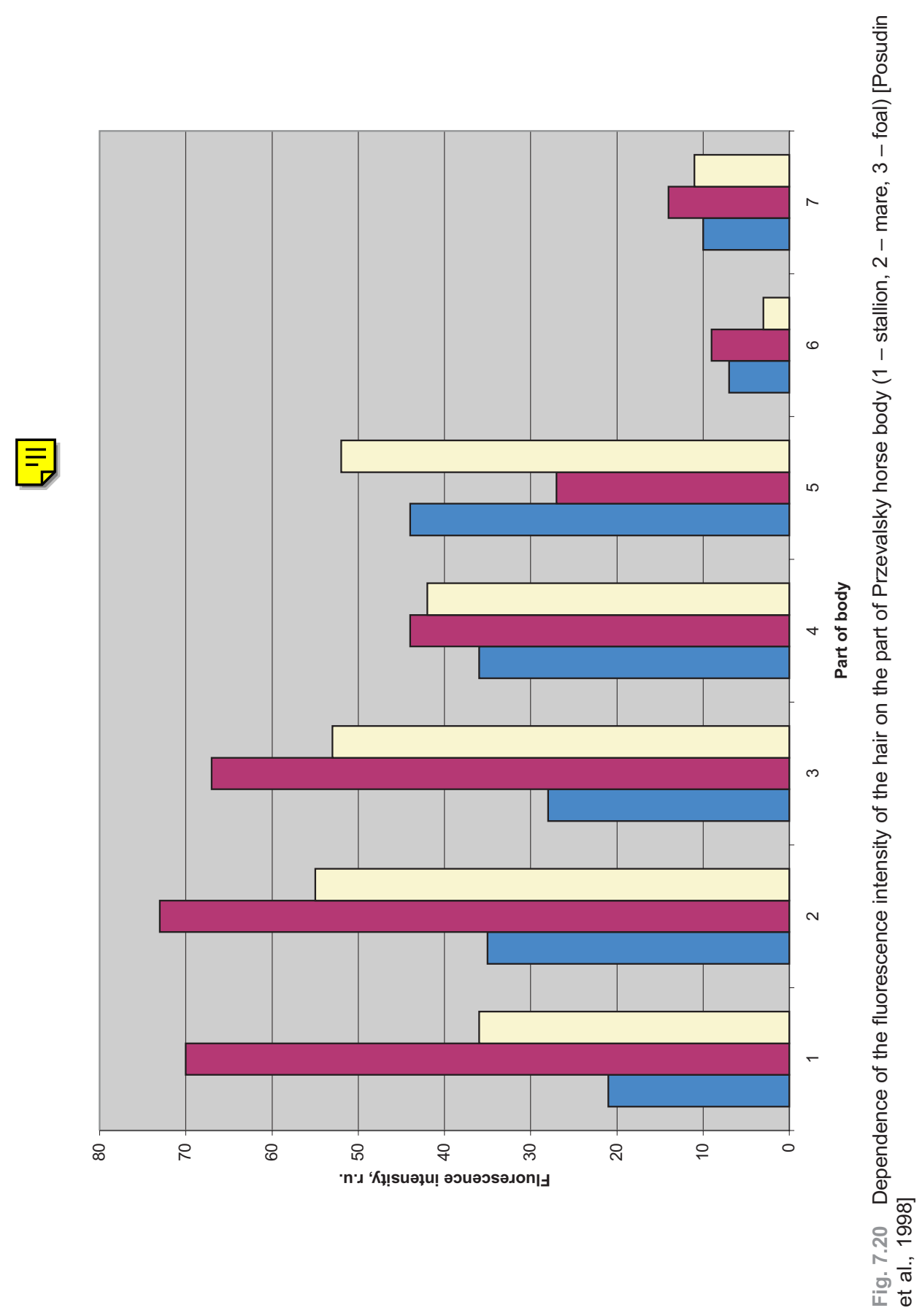


Table 7.7 Effect of non-additive domination on genotype signs for the Przevalsky horse family

\begin{tabular}{l|r|r|l|c|c}
\hline $\begin{array}{c}\text { Part of } \\
\text { Body }\end{array}$ & $k$ & $\Delta$ & $\begin{array}{c}\text { Formation of Genetic } \\
\text { Level of Descendant }\end{array}$ & $\begin{array}{c}\text { Coefficient } \\
\text { of Regression } \\
\text { for Mare }\end{array}$ & $\begin{array}{c}\text { Coefficient of } \\
\text { Regression } \\
\text { for Stallion }\end{array}$ \\
\hline Cheek & 2.22 & -22.00 & $F=(M+2.22 S) / 3.22$ & 0.31 & 0.69 \\
Back & 0.96 & 1.00 & $F=(M+0.96 S) / 1.96$ & 0.51 & 0.49 \\
Side & 0.44 & 17.50 & $F=(M+0.44 S) / 1.44$ & 0.69 & 0.31 \\
Croup & -3.30 & 38.50 & $F=(M-3.3 S) /-2.30$ & -0.43 & 1.43 \\
Stomach & 0.27 & 5.50 & $F=(M+0.27 S) / 1.27$ & 0.790 & 0.21 \\
Mane & -1.34 & -10.50 & $F=(M-1.34 S) /-0.34$ & -2.90 & 3.90 \\
Tail & 7.00 & -30 & $F=(M+7 S) / 1.70$ & 0.59 & 4.12 \\
\hline
\end{tabular}

Method of microspectrofluorometry of horse hair can be used for quantitative evaluation of level and character of pigment distribution within hair, studying internal structure of hair, diagnostics of state of horse under influence of feeding conditions and effects of external factors, and degree of heredity [Posudin, 1988].

It is very informative to retrace the level of the heredity of hair fluorescence by several generations of animals.

\subsubsection{Laser Diffractometry of Animal Hair and Nasolabial Mirror}

Hair of Sportive Horses. Laser diffractometry of horse hair, which was taken from different parts of the animal body, made it possible to relate parameter $l_{m}$ (interval between diffraction maxima) and $d$ (diameter of hair). The application of dispersive analysis of two-factor complex ( $i=$ part of body, $j=$ colour of hair) testifies to the fact that the calculated criteria $F_{i}, F_{j}$ and $F_{i j}$ are higher than the critical value $F_{c}$ for the level of significance $P=0.05$ and $P=0.01$ (Table 7.8).

Table 7.8 Results of application of dispersive analysis of two-factor complex ( $i$ - part of body, $j$ - colour of hair)

\begin{tabular}{l|c|c|c|c}
\hline Source of Variation & $\begin{array}{c}\text { Degree of } \\
\text { Freedom }\end{array}$ & $\begin{array}{c}\text { Actual Factor } \\
F_{a}\end{array}$ & $\begin{array}{c}\text { Critical Factor } \\
F_{c}(P=0.05)\end{array}$ & $\begin{array}{c}\text { Critical Factor } \\
F_{c}(P=0.01)\end{array}$ \\
\hline Factor $i$ (part of body) & 7 & $F_{i}=25.987$ & 2.200 & 3.000 \\
Factor $j$ (colour of hair) & 2 & $F_{j}=19.487$ & 3.200 & 5.000 \\
$\begin{array}{l}\text { Common action of two } \\
\text { factors } i j\end{array}$ & 14 & $F_{i j}=13.108$ & 1.800 & 2.200 \\
Residue & 60 & $F_{z}=1$ & - & - \\
\hline
\end{tabular}


Independent and common effects of both factors and their interaction are statistically significant as soon as $F>F_{c}$.

Vibrissa of Calves and Heifers. The results of laser diffractometry of the vibrissa of bull calves and heifers were compared with the weight of the calves: 371 heads were distributed to nine weight ranks (from $26-27 \mathrm{~kg}$ to 42-43 kg). Analysis of diffraction patterns (Figs. 7.21-7.28) demonstrated that the dominating weight groups were [Posudin and Trofimenko, 1994]: $28-29$ and $30-31 \mathrm{~kg}$ in heifers; and 30-31 and 32-33 kg in bull calves. The corresponding thickness of vibrissa consists of: $100-150 \mu \mathrm{m}$ $(\Delta l=40-60 \mathrm{~mm})$ for $28-29 \mathrm{~kg}$ group of heifers; $85-120 \mu \mathrm{m}(\Delta l=50-70$ $\mathrm{mm})$ for $30-31 \mathrm{~kg}$ group of heifers and bull calves; and $80-122 \mu \mathrm{m}(\Delta \mathrm{l}=$ $49-75 \mathrm{~mm}$ ) for $32-33 \mathrm{~kg}$ group of bull calves.

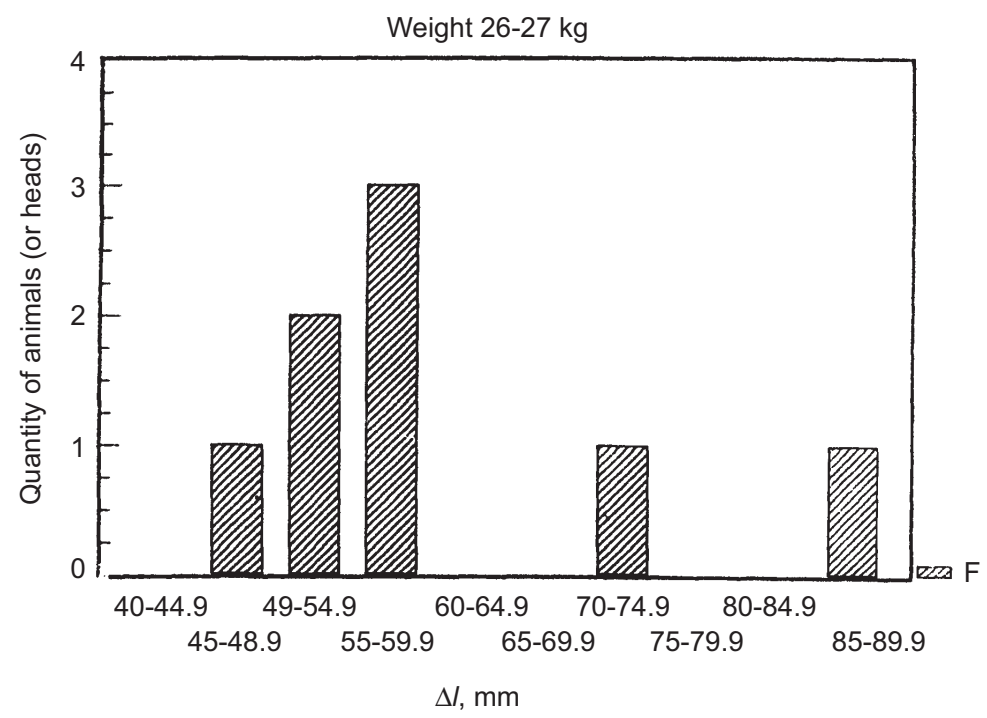

Fig. 7.21 Results of laser diffractometry of the vibrissa of heifers of weight $26-27 \mathrm{~kg}$. Here: $\Delta l$ - distance between diffraction maxima [Posudin et al., 1993]

Thus, the method of laser diffractometry can possibly help to estimate the relationship between the hair thickness, weight and sex of the animals.

Another application of laser diffractometry is related to the analysis of the hair orientation. It is known that the angle of hair inclination in animals depends on the environmental temperature: this angle increases from $28^{\circ}$ to $63^{\circ} \mathrm{C}$ with the decrease in temperature from $+11^{\circ}$ to $-24^{\circ} \mathrm{C}$; 
128 Practical Spectroscopy in Agriculture and Food Science

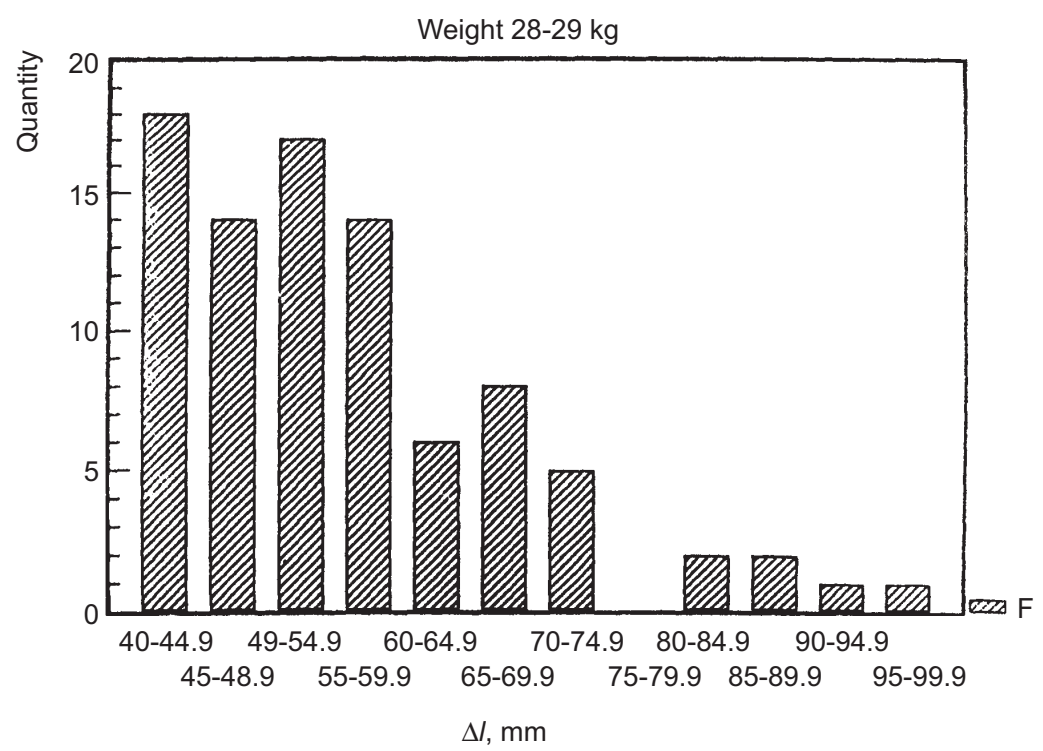

Fig. 7.22 Results of laser diffractometry of the vibrissa of heifers of weight $28-29 \mathrm{~kg}$ [Posudin et al., 1993]

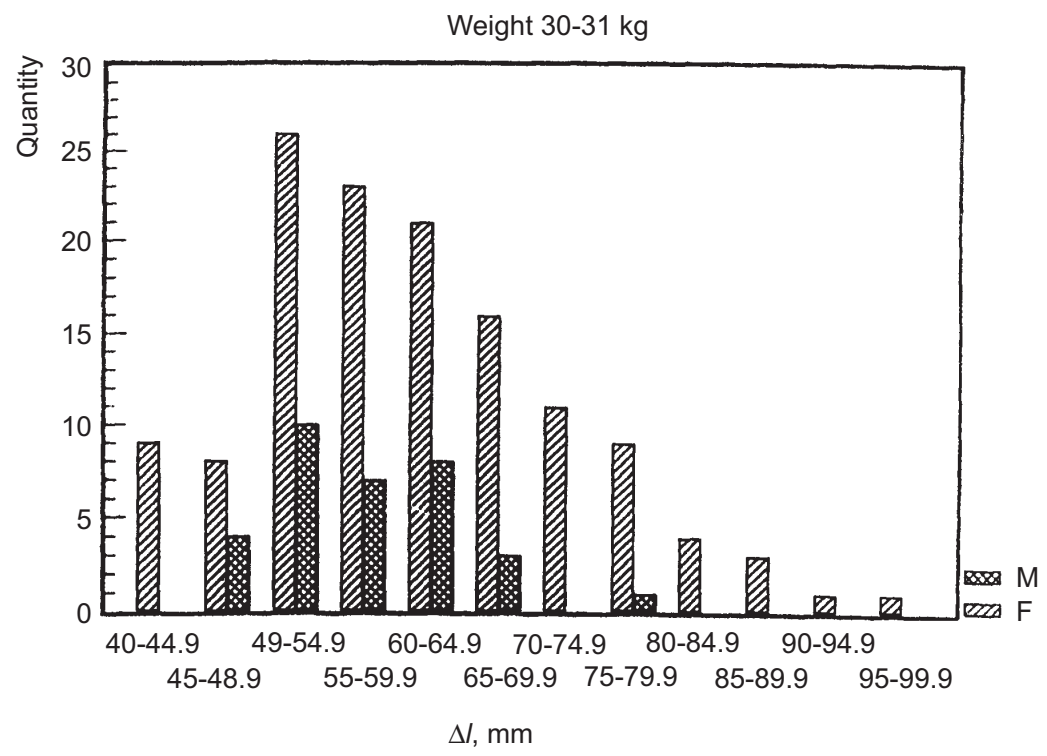

Fig. 7.23 Results of laser diffractometry of the vibrissa of bull calves and heifers of weight 30-31 kg. Here and in Figs. 7.24, 7.25, and 7.28: $M$ - calves, $F-$ heifers, $\Delta /-$ distance between diffraction maxima [Posudin et al., 1993] 


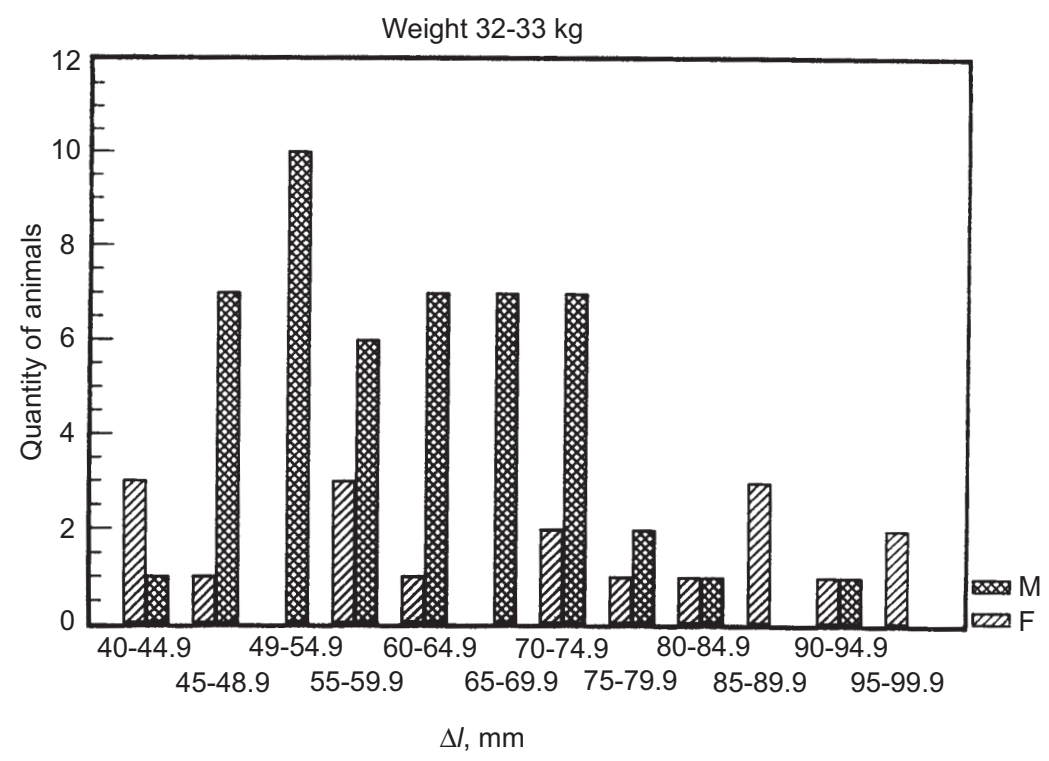

Fig. 7.24 Results of laser diffractometry of the vibrissa of bull calves and heifers of weight 32-33 kg [Posudin et al., 1993]

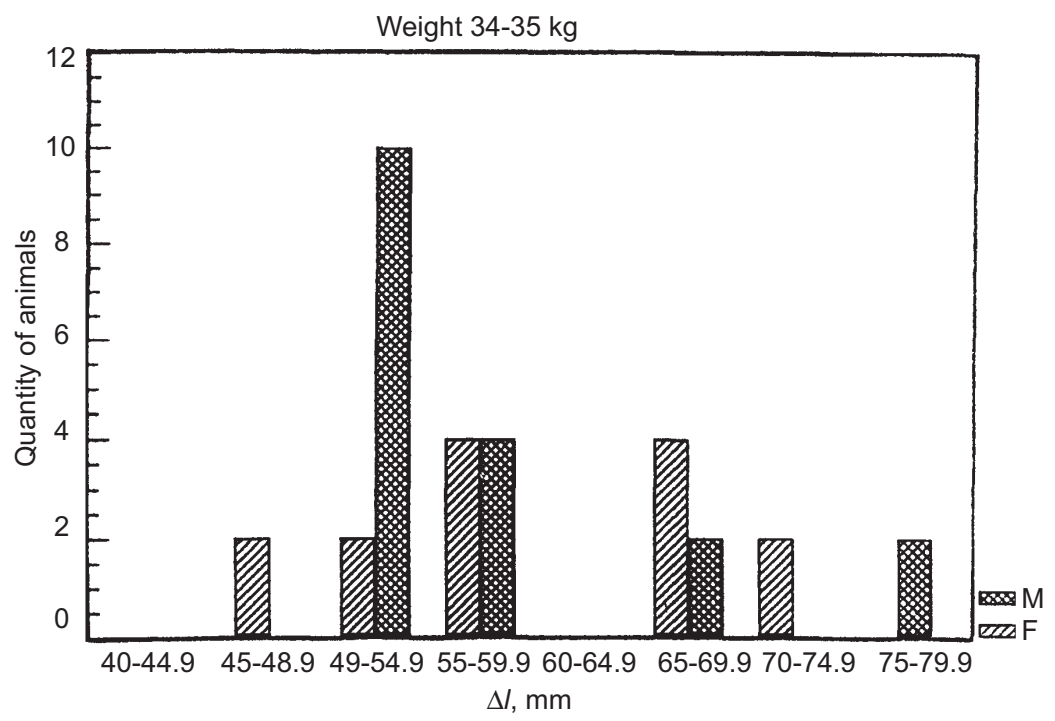

Fig. 7.25 Results of laser diffractometry of the vibrissa of bull calves and heifers of weight $34-35 \mathrm{~kg}$. $M$ - calves, $F-$ heifers, $\Delta l$ - distance between diffractional maxima [Posudin et al., 1993] 
130 Practical Spectroscopy in Agriculture and Food Science

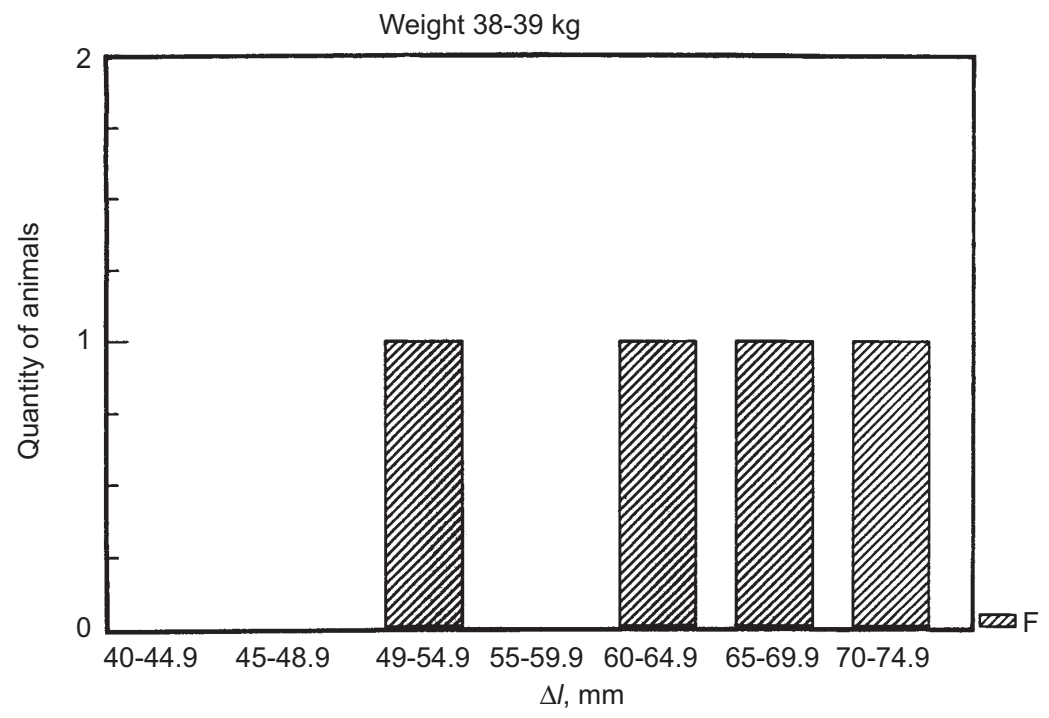

Fig. 7.26 Results of laser diffractometry of the vibrissa of heifers of weight $38-39 \mathrm{~kg}$ [Posudin et al., 1993]

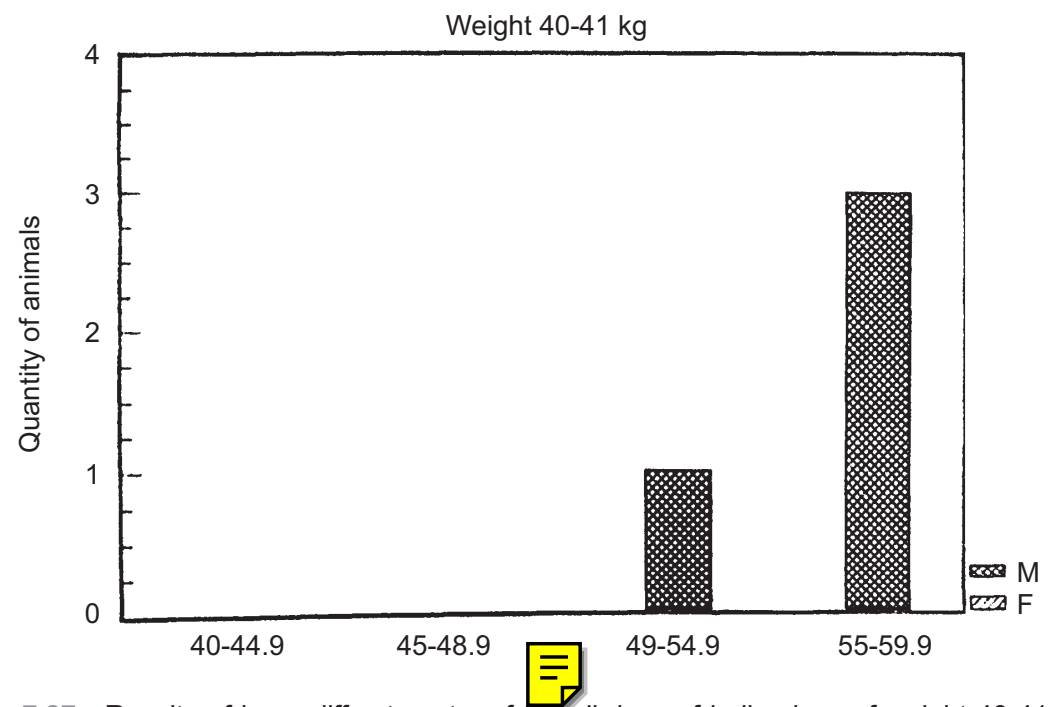

Fig. 7.27 Results of laser diffractometry of vibrissa of bull calves of weight $40-41 \mathrm{~kg}$ [Posudin et al., 1993] 


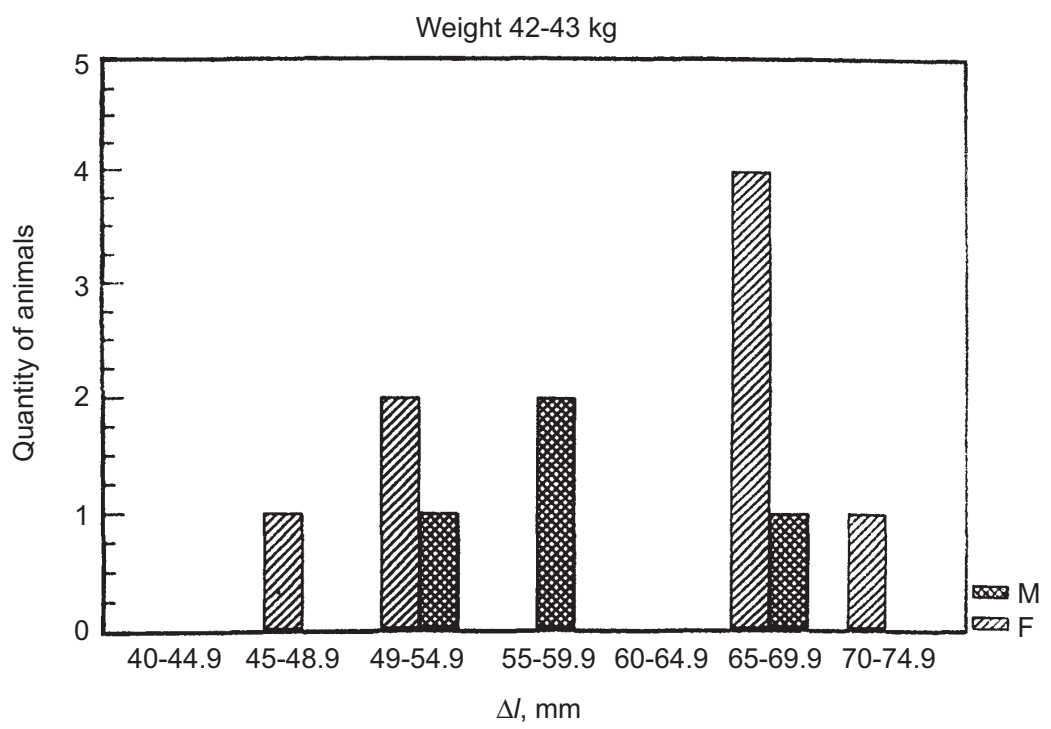

Fig. 7.28 Results of laser diffractometry of the vibrissa of bull calves and heifers of weight 42-43 kg [Posudin et al., 1993]

the thickness of the hair layer changes correspondingly from 11 to $21 \mathrm{~mm}$. However, the change in the angle of inclination provokes the alteration of spatial orientation of the diffraction pattern (Fig. 7.29). The measurement of the angular position of the hair and diffraction pattern helps to evaluate the effects of thermal stresses on farm animals.

\subsection{SELECTION IN CATTLE-BREEDING}

Nasolabial Mirror of the Cattle. The structure of nasolabial mirror of the cattle is characterized by a specific pattern which presents a peculiar fingerprint of the animal. There are several different types of dermatoglyphs such as crown, twig, ear, grain and their combinations [Trofimenko et al., 1990]. The analysis of the structure of this dermatoglyph is promising from the point of view of early prognosis of the animal.

The differentiation of the cattle is performed by the analysis of genealogical properties of external, morphological, biochemical, and physiological indices.

The skin-coat of the animal presents the important factor which could be used for early prognosis of the main trends of cattle development. The thickness of the skin, its density and friability depend on the sex, age, constitution of the animal, conditions of feeding and maintenance. 


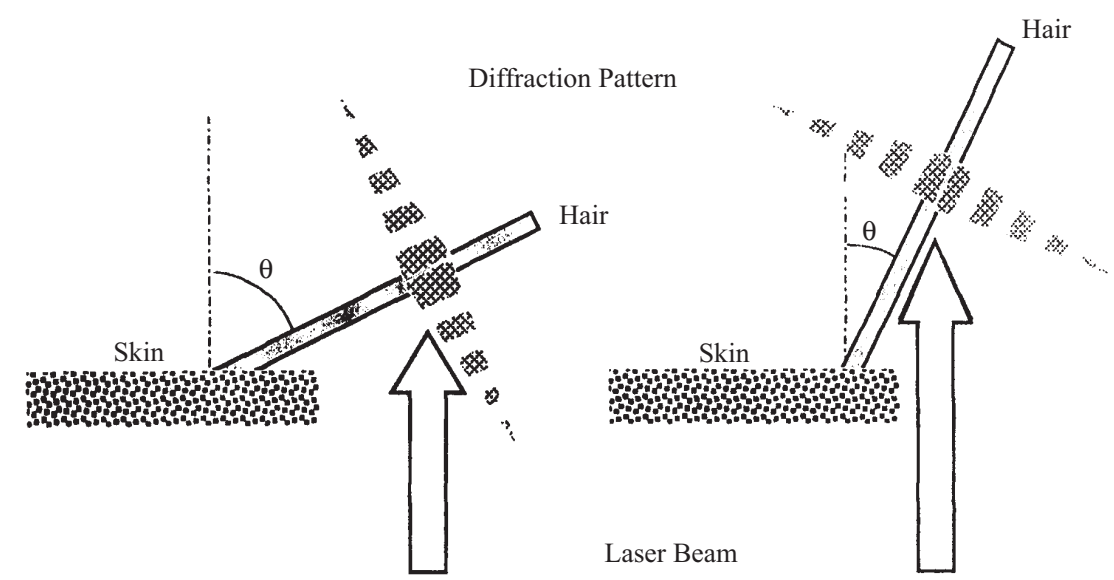

Fig. 7.29 Dependence of spatial orientation of diffraction pattern on the inclination angle $\theta$ of the hair [Posudin, 1998]

Considering the automation of laser systems of dermatoglific identification and classification, the first variant [Trofimenko et al., 1990] consists of laser, Fourier-objective, optical system (diaphragm and mirrors), videocamera and system of elaboration of data. The radiation of laser passes through the slide with the picture of nasolabial structure (Fig. 7.30). The analysis of spatial structure of the optical image can give the information about of the sex of the individual.

The second approach is based on laser diffractometry of nasolabial mirror [Posudin et al., 1998]. The procedure of measurements includes the interaction of laser radiation with the quasi-periodical structure of the mirror, analysis of diffraction pattern (Fig. 7.31) and identification of animal diversity.

\subsection{LASER CONTROL OF BIRD FEATHERING}

Morphology of bird feathering, its anatomical, histological properties and colour depend on the state, sex and age of the bird, the external factors, season of year, conditions of maintenance and feeding of the birds.

The structure of the bird feather is presented in Fig. 7.32a. The main parts of the feather are pivot (scapus) and large fan (vexillum). Scapus consists of calamus and rachis. Vexillum includes rami (singular: ramus), the central shaft of feather barbule that is attached to the rachis, and 


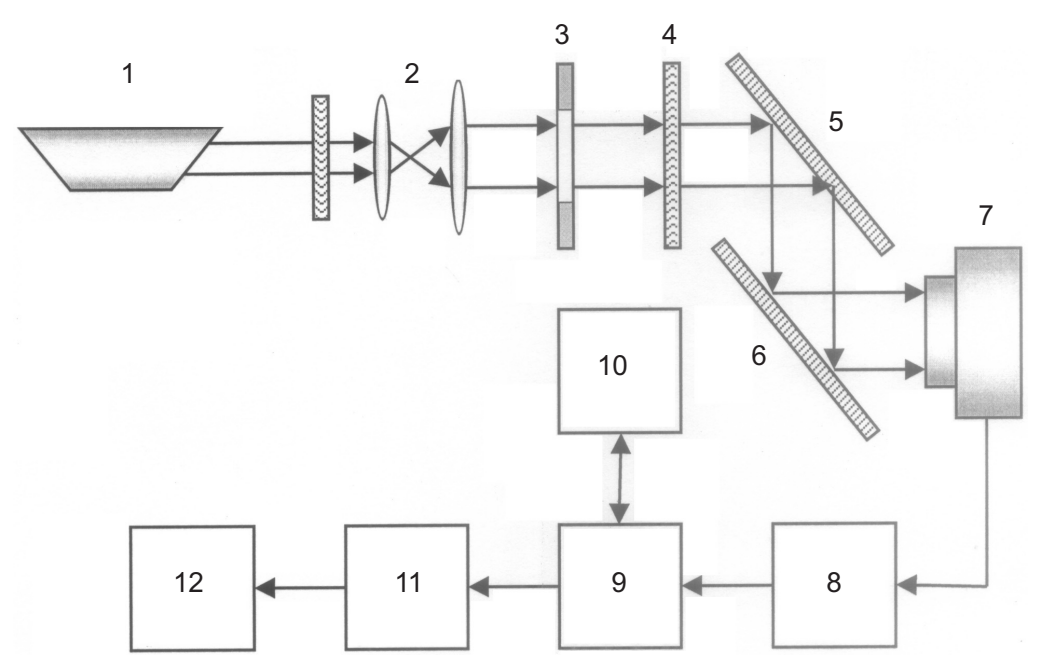

Fig. 7.30 Automated laser system of dermatoglific identification and classification. 1 laser; 2 - Fourier-objective; 3 - diaphragm; 4 - film; 5, 6 - mirrors; 7 - videocamera; 8 input controller; 9 - computer; 10 - control panel; 11 - block of conjugation; 12 - display [Trofimenko et al.,1990]

dozens of small radi (singular: radius) that extend out from either side of the rami.

The estimation of morphology of the feather is performed as per the following parameters [Svetozarov and Shtraikh, 1939]: the length $A$ of the feather part of vexillum; the length $B$ of the lower part of vexillum; the total length $A+B$ of vexillum; the length $C$ of scapus; the width $D$ of vexillum; the diameters $d_{1}, d_{2}, d_{3}$ of scapus in different places; the points $E, F, G, H$ and $O$, which are situated $5 \mathrm{~mm}$ from the scapus and where the number of rami is determined.

These parameters can be used as the taxonomical indices which are related to the species and race of the bird (Table 7.9).

As a whole, the feather presents the quasi-periodical structure which can be considered a peculiar diffraction grating. Laser radiation can interact with such a structure and produce a diffraction pattern (Fig. $7.32 b)$. The relative intensity and spatial position depends on the geometry of the feather (diameter of the ramus and radius) which reflects the healthy status of the bird, diseases, regime of feeding, etc. [Posudin and Lepeshenkov, 1991]. 
134 Practical Spectroscopy in Agriculture and Food Science

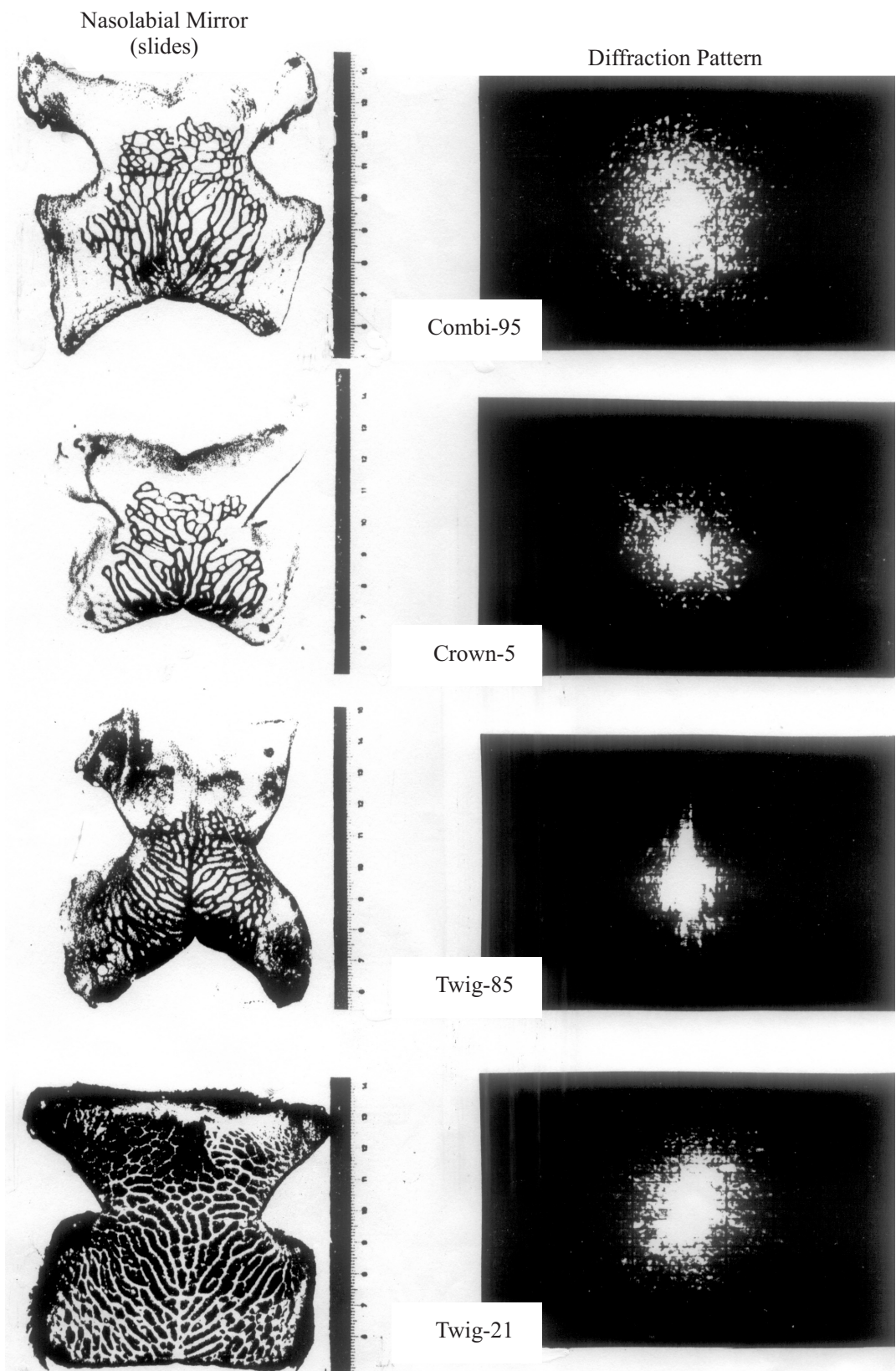

Fig. 7.31 The interaction of laser radiation with the quasi-periodical structure of the nasolabial mirror of the cattle, analysis of diffraction pattern and identification of animal diversity [Posudin et al., 1998] 


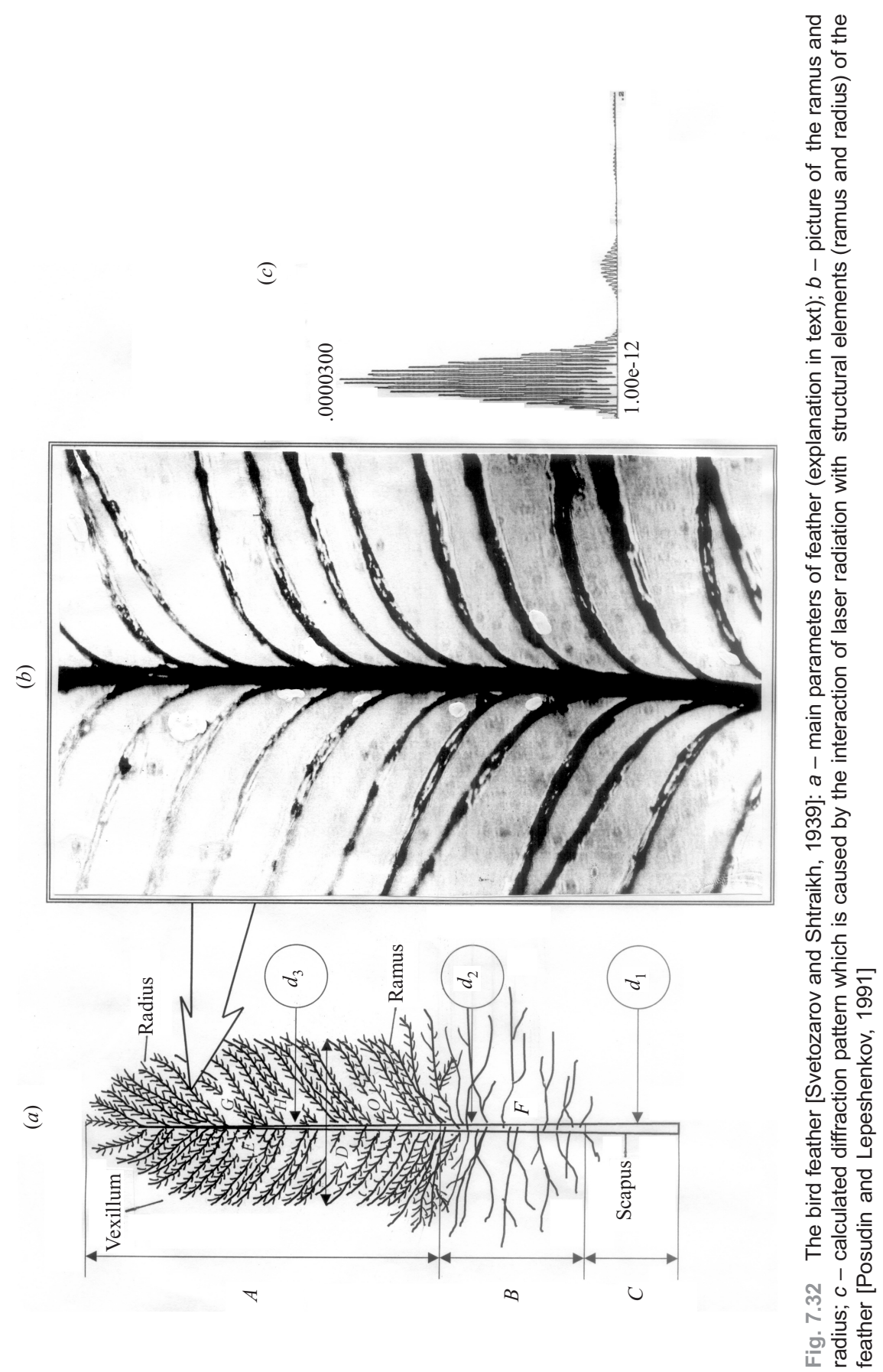




\section{Practical Spectroscopy in Agriculture and Food Science}

Table 7.9 Specific and racial distribution in the size of the main feather parts [Svetozarov, Shtraikh, 1939]

\begin{tabular}{l|c|c|c|c}
\hline \multirow{2}{*}{ Indices } & \multirow{2}{*}{ Goose } & Ducks & Ducks & \multirow{2}{*}{ Hen } \\
\cline { 3 - 4 } & & Pekin & Rouen & \\
\hline Whole length of feather, mm & 60 & 55 & 46 & 70 \\
\hline Length, mm & 10 & 8 & 10 & 6 \\
- pivot & 90 & 92 & 90 & 94 \\
- fan & 64 & 68 & 68 & 44 \\
- feather part of fan & 28 & 25 & 22 & 59 \\
- down part of fan & 52 & 45 & 42 & 38 \\
\hline Width of fan, mm & & & & \\
\hline
\end{tabular}

\subsection{SUMMARY}

The dependence of transmittance of horse hair on the illuminance $E$ was investigated in the author's experiments. It was shown that the curves $\tau=$ $f(E)$ depend strongly on the colour and part of the animal body; each curve is characterized by a linear part of dependence $\tau=f(E)$, and the region of saturation where $\tau \rightarrow 1$. The hair of grey colouration has maximal transmission, while minimal transmission is typical for the hair of black colouration. The linear dependence $\tau=f(E)$ can be used for quantitative estimation of colour of hair-covering of horses.

The fluorescence intensity of the hair depends on the part and colour of the body (sportive horses, ponies and Przevalsky horse). Method of microspectrofluorometry of the horse hair can be used for quantitative evaluation of level and character of pigment distribution within the hair, studying internal structure of the hair, diagnostics of state of the horse under influence of feeding conditions, and effects of external factors.

It is very attractive to use fluorescence parameters of the animal (pony and Przevalsky horse) hair as genotypic signs of animal diversity for determining the degree of heritability.

\subsection{REFERENCES}

Bannikov, A.C. 1958. Distribution geographique et biologie du cheval sauvage et chameau de Mongolie. Mammalia, 22: 152-160.

Born, M. and Wolf, E. 1970. Principles of Optics. 4th ed. Pergamon Press, Oxford, London, N.Y.

Combs, D.K. 1987. Hair analysis as an indicator of mineral states of livestock. J. Amer. Sci. 65(6): 1753-1758.

Durst, W. 1963. Exterior of Horse. Moscow-Leningrad. Selkhozgiz. p 552. 
Gopka, B.M., Kalantar, O.A. and Pavlenko, P.M. 1989. Horses in Àgriculture. Kiev, Urozhai, p 150.

Groves, C.P. 1986. The taxonomy, distribution and adaptations of recent Equids. In: Equids in the Ancient World. R.H. Meadow and H.P. Uerpmann, eds. Dr Ludwig Reichert Verlag, Wiesbaden, Germany, p.11-65.

Ioganson, I., Rendel, Y. and Gravert, O. 1970. Genetics and Breeding of Domestic Animals. Moscow, Kolos, Russia (or USSR) p 351.

Klimov, V.V. 1990. The Przevalsky Horse. Agropromizdat, Moscow, p 253.

Kostaneski, W. 1970. Choroby wlosów. Warsaw, Poland.

Plokhinsky, N.A. 1964. Heritability. Novosibirsk, Siberian Branch of Academy of Science, p 194.

Poliakov, I.S. 1881. Przewalski's Horse (Equus przwalskii n. sp.). Izvestia Russki Geographicheski obschestva, St. Petersburg, 17: 1-20.

Posudin, Yu.I. 1995. Biophysics. Urozhaj, Kiev. p 222.

Posudin, Yu.I. 1988. Lasers in Agriculture, Science Publishers, Inc. Enfield, NH, USA. p 188.

Posudin, Yu.I., Chervinsky, L.S., Yasynetska, N.I. and Zharkykh, T.L. 1998. Investigation of fluorescence characteristics of Przevalsky horse hair. Sci. Herald of Nat. Agr. Univ. 4: 3-9.

Posudin, Yu.I., Gopka, B.M. and Zatvarsky, B.M. 1991. Optical, spectral and laser methods of investigation of horse hair cover. Biol. Sciences. Depon VINITI, N257-B91, p 28.

Posudin, Yu.I. and Lepeshenkov, V.F. 1991. Optical and laser methods of poultry-breeding control. St.-Petersbourg Agr. Univ. Publ. 1: 59-64.

Posudin, Yu.I., Trofimenko, A.L., Koval, Y.M. and Palecha, N.P. 1993. Spectral characteristics of animals' hair as genetic indices. Cytology and Genetics. 27: 74-77.

Posudin, Yu.I. and Trofimenko, A.L. 1994. Laser diffractometry of calves hair. Bull. Agr. Science 9: 85-89.

Posudin, Yu.I., Trofimenko, A.L. and Vinnichuk, D.T. 1998. Method of identification of animal features through the structure of nasolabial mirror. Patent of Ukraine 25105A; N 95041577, 1998.

Seiji, M. 1973. On the melanization process of melanosomes. Pigment Cell. 1: 39-46.

Sokolov, E.V. and Zhenevskaya, R.P. eds. 1988. Guidance to study skin-covering of mammals. Moscow, Nauka, p 280.

Svetozarov, E.A. and Shtraikh, G.G. 1939. Comparative morphology of feather in agricultural birds. Izv. AN USSR. Ser. Biol (Russian). 5: 800-822.

Trofimenko, A.L. and Posudin, Y.I. 1998. Description of polyphenism of features during selection of cattle. Sci. Herald of Nat. Agr. Univ. 10: 55-61.

Trofimenko, A.L., Vinnichuk, D.T. and Yakovlev, S.G. 1990. Forecast and selection of the cattle. In: Agriculture. Series Cattle-Breeding and Veterinary. Kiev, p 36.

Wojcikowska-Soroczynska, M., Radosz A. and Fiedorczuk E. 1987. Quantitative appearance of pigments in hairs of sheep and of selected animal species. Ann. Warsaw Agr. Univ.-SGGW-AR Animal Science, 21: 17-21. 


\section{Spectroscopic Analysis of Agronomic Plants}

\subsection{LASER SPECTROFLUOROMETRY OF AGRONOMIC PLANTS}

\subsubsection{Photosynthesis and Fluorescence of Chlorophyll}

Photosynthesis is the conversion of light energy into stabilized chemical energy through light absorption by a pigment molecule, excitation energy transfer and the photochemical reaction in photosystem PSII. The process of deexcitation of the absorbed light energy is related to heat emission and chlorophyll fluorescence. Fluorescence is the radiative process which is based on the transition between electronic states of the same multiplicity; usually it occurs from the ground vibrational state of the first electronic singlet state $S_{1}$ to various vibrational levels in ground singlet state $S_{0}$, and is accompanied by the emission of photons. The light absorbed by the accessory pigments (chlorophyll $b$ and carotenoids) is transferred to chlorophyll $a$. That is why the primary processes of photosynthesis are reflected by chlorophyll $a$ fluorescence. The yield of chlorophyll fluorescence depends on numerous factors in a very complex manner; the inverse relationship between in vivo chlorophyll fluorescence and photosynthetic activity of leaves can be practically used to study leaf development and detection of stress effects in green plants. Either leaf anatomy and morphology [Sestak, 1985; Sestak and Siffel, 1988; Lichtenthaler and Rinderle, 1988], or various stress conditions [Subhash and Mohanan, 1994] can govern the rate of photosynthesis and provoke the changes of fluorescence parameters. Fluorescence emission can also be studied as a means of assessing chlorophyll content, which in 
some plant products is related to maturity or certain quality attributes [Kays and Paull, 2004].

\subsubsection{Fluorescence Properties of Agronomic Plants}

The fluorescence emission spectrum of a green leaf is characterized by a maxima at 735-740 nm and 685-690 nm (red part of spectrum) and at 440-450 nm (blue part); the leaves of some plants demonstrate a shoulder near 520-530 nm (green part). According to recent theory about the character of the fluorescence spectrum of a green leaf, the red maxima originate from chlorophyll $a$. The experimental investigations showed the presence of a number of native forms of chlorophyll. There are the fluorescence bands at room temperature near 672, 677, 682, 687, $\underline{693-695}, 700,720-725, \underline{735}, 750-760, \underline{800-820}$ (the underlined figures correspond to the intense bands) (Kochubey, 1986). The appearance of these bands is related to the process of aggregation of pigment molecule and formation the chlorophyll-protein complexes.

It is not defined which compounds are responsible for the blue and green parts of the fluorescence spectrum. There is an opinion [Lang et al., 1991; Lichtenthaler et al., 1991; Goulas et al., 1990; 1991] that various phenolic plant constituents in the vacuoles and cell walls of both the epidermal layer and the mesophyll cells such as the coumarines aesculetin and scopoletin, with a very high fluorescence yield, and cinnamic acids and derivatives, with a lower yield (such as caffeic and chlorogenic acid, sinapic acid and catechin) are responsible for the bluegreen fluorescence. Some investigators [Chappelle et al., 1991] consider that $\beta$-carotene, nicotinamide adenine diphosphate (NADPH), and vitamin $K_{1}$ participate in fluorescence at $440 \mathrm{~nm}$. However, purified $\beta$ carotene does not show any blue or green fluorescence [Lang et al., 1991]; the yield of blue fluorescence $\equiv$ JAPDPH is extremely low [Lang et al., 1992]; the phylloquinone, $K_{1}, t$ esces only under decomposition after intense UV exposure [Interschick-Niebler and Lichtenthaler, 1981].

The red chlorophyll fluorescence with two maxima near $690 \mathrm{~nm}$ and $740 \mathrm{~nm}$ emanates from the chlorophyll $a$ in the chloroplasts of the leaves' mesophyll cells [Stober and Lichtenthaler, 1993].

The shape and intensity of the main bands in fluorescence emission spectrum of green leaves depend on the pigment content which may vary due to such factors as the growth phase of the plants, natural stresses (high light, heat, water shortage and mineral deficiency) and anthropogenic stresses (agrochemical treatment, air pollution, ozone, acid rains, heavy metals and ultraviolet irradiation). 


\section{Practical Spectroscopy in Agriculture and Food Science}

\subsubsection{Plant Samples}

The following were investigated during fluorescence measurements:

- Leaves of cereals: rye, barley (winter and spring sorts), wheat (winter and spring sorts, soft and stiff species), hybrids amphidiploid, corn.

- Leaves of vegetables: cucumber, tomato, haricot, vegetable marrow, pumpkin, pepper, sunflower;

- Vegetables: vegetable marrow, tomato, haricot, horse-radish, sorrel.

- Berries: currants and raspberry-cane. The sprouts of the cultures were grown in Petri dishes at room temperature in the period of three to eight days. The length of the sprouts varied from 5 to 25 $\mathrm{mm}$. The list of etiolated sprouts (winter and spring sorts) of cereals is given in Table 8.1, etiolated sprouts (winter soft sorts) of cereals in Table 8.2, and of yellow stems (winter and spring sorts) of cereals in Table 8.3.

\subsection{INSTRUMENTATION}

The schematic representation of laser spectrofluorometer (which was worked out by the author of this work) is given in Fig. 8.1. Nitrogen laser 1 was used as the source of excitation of chlorophyll fluorescence. The main characteristics of this laser are: wavelength of generation $337 \mathrm{~nm}$, frequency of pulse repetition $50 \mathrm{~Hz}$, an average power output of $3 \mathrm{~mW}$, duration of pulse about $10 \mathrm{~ns}$, and divergence of beam $3 \cdot 10^{-3}$ radians.

The laser radiation is directed through the semitransparent glass plate 2 to the sample 3 (a single intact leaf) and to the fluorescent standard 4 (a solution of fluorescent). Either the sample, or the cell with fluorescent standard is oriented at an angle of $45^{\circ}$ to the laser beam in order to escape non-desirable absorption of laser radiation by the sample (or standard) volume.

The fluorescence emission of the leaf (or the standard), which is collected by a spherical mirror 5, passes through the cut-off filter 6 , and is focused on the entrance slit 7 of monochromator 8 . The dispersive element (prism) 9 of this monochromator is linked with the electrical motor 10, which provides the rotation of the prism and selection of the wavelength. The intensity of fluorescence is detected by photomultiplier 11; the electrical signal of it is amplified and recorded by the readout system (amplifier 12 and recorder 13). 
Spectroscopic Analysis of Agronomic Plants

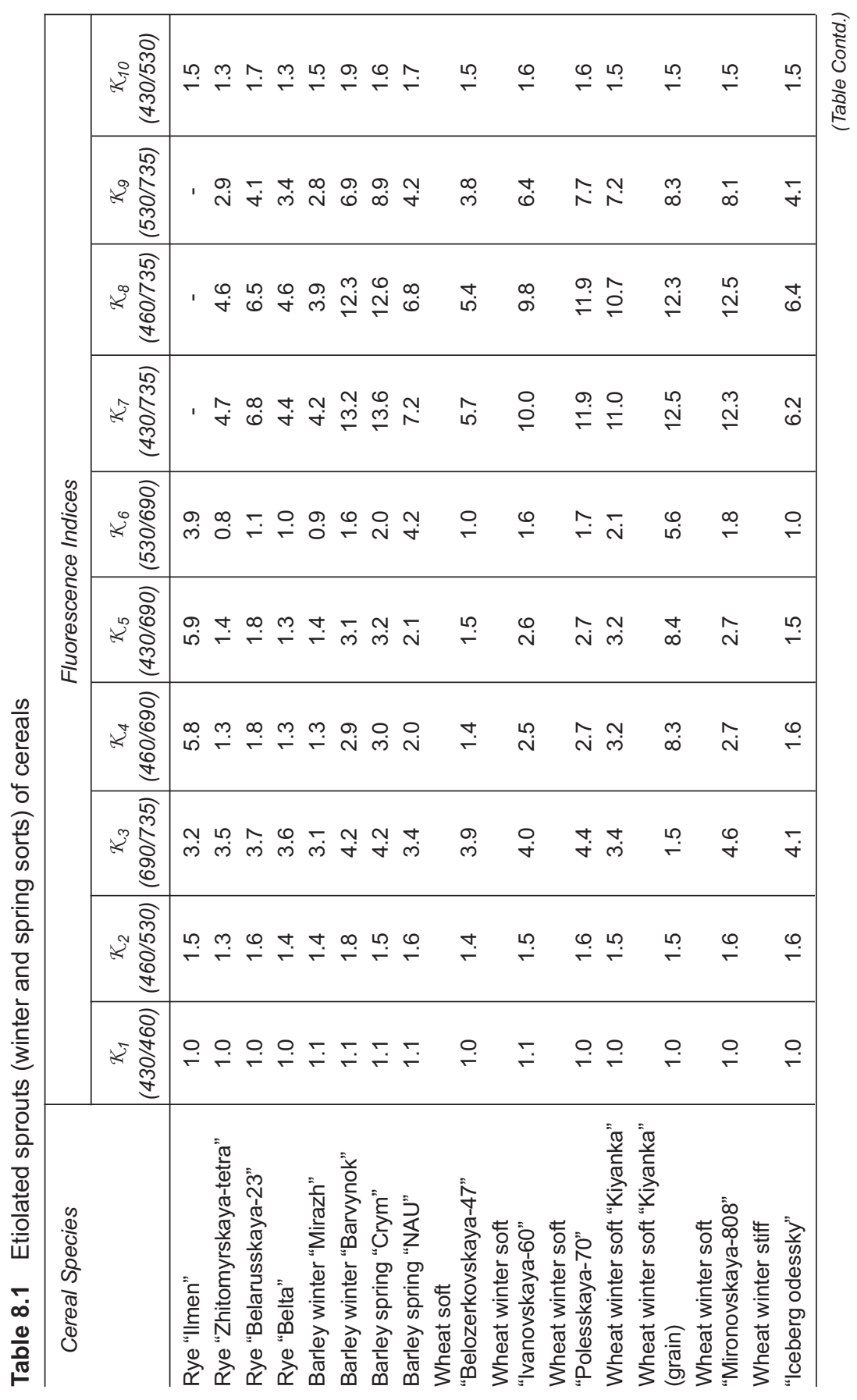


142 Practical Spectroscopy in Agriculture and Food Science

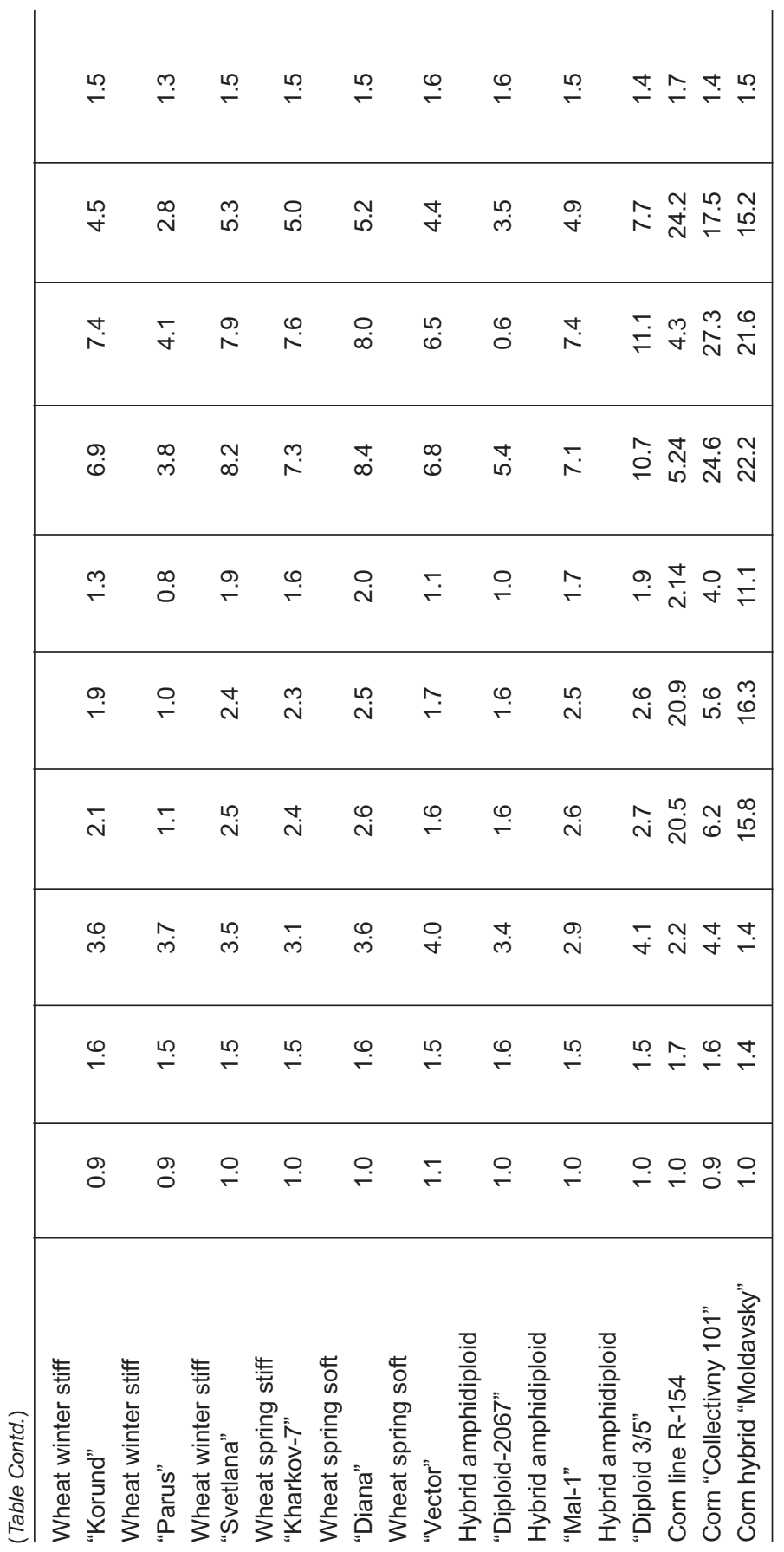


Table 8.2 Fluorescence indices of different species of etiolated sprouts of winter soft wheat

\begin{tabular}{l|c|c|c}
\hline Species & $\begin{array}{c}\mathcal{K}_{2} \\
(460 / 530)\end{array}$ & $\begin{array}{c}\mathcal{K}_{3} \\
(690 / 735)\end{array}$ & $\begin{array}{c}\mathcal{K}_{4} \\
(460 / 690)\end{array}$ \\
\hline "Belozerkovskaya-47" & 1.4 & 4.4 & 1.0 \\
& 1.7 & 4.1 & 1.3 \\
& 1.4 & 3.4 & 0.8 \\
& 1.5 & 4.2 & 1.6 \\
"Polesskaya-70" & 1.5 & 4.3 & 1.6 \\
& 1.4 & 3.9 & 1.4 \\
& 1.5 & 4.3 & 2.0 \\
& 1.7 & 3.3 & 5.9 \\
& 1.6 & 4.1 & 3.1 \\
"Kiyanka" & 1.6 & 4.4 & 4.9 \\
& 1.6 & 3.8 & 4.7 \\
"Ivanovskaya-60" & 1.6 & 4.4 & 2.7 \\
& 1.5 & 4.0 & 3.6 \\
& 1.6 & 4.4 & 1.9 \\
& 1.5 & 3.4 & 3.2 \\
"Mironovskaya-808" & 1.6 & 4.6 & 2.9 \\
& 1.8 & 4.6 & 2.6 \\
& 1.9 & 4.4 & 2.8 \\
\hline
\end{tabular}

\subsection{RESULTS OF LASER SPECTROFLUOROMETRY OF A SINGLE LEAF}

\subsubsection{Fluorescence Indices}

The following fluorescence intensity ratios have been used as the vegetation indices [Ivanitskaya and Posudin, 1989]: $\mathcal{K}_{1}=430 / 460, \mathcal{K}_{2}=$ $460 / 530, K_{3}=690 / 735, K_{4}=460 / 690, K_{5}=430 / 690, K_{6}=530 / 690, K_{7}=$ $430 / 735, K_{8}=460 / 735, K_{9}=530 / 735, K_{10}=430 / 530$.

\subsubsection{Fluorescence Emission Spectra during Greening of Etiolated Leaves}

The values of the fluorescence indices for the etiolated sprouts of cereals (soft and stiff species, winter and spring sorts) are presented in Tables 8.1 and 8.2. It is shown that the values of $\mathcal{K}_{1} \approx 1, \mathcal{K}_{2}-\mathcal{K}_{10} \approx 1.5$, and $\mathcal{K}_{3}$ $\approx 3-4$ are constant. However, the ratio of blue/red bands is different. 
144 Practical Spectroscopy in Agriculture and Food Science

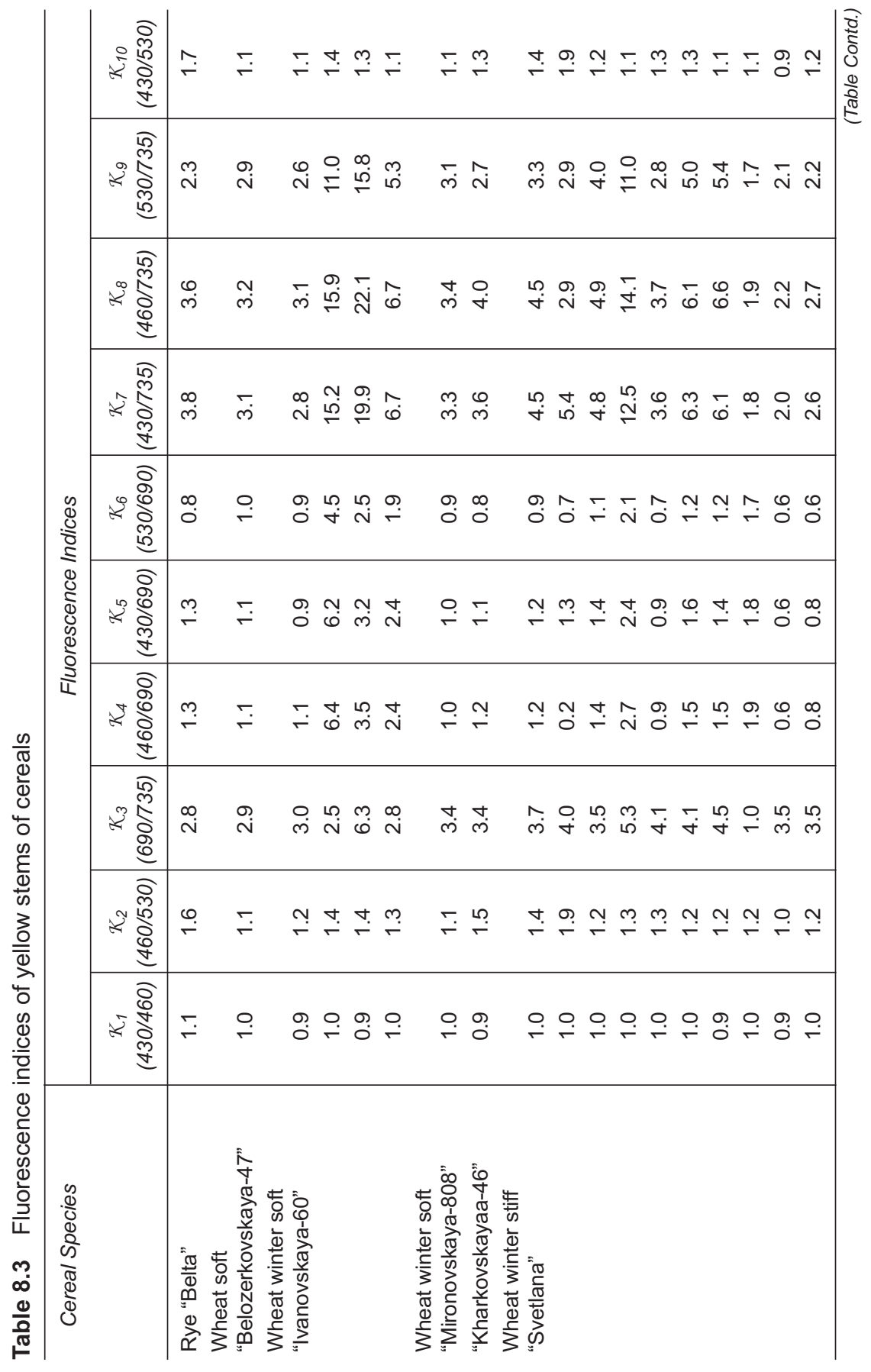


Spectroscopic Analysis of Agronomic Plants $\mathbf{1 4 5}$

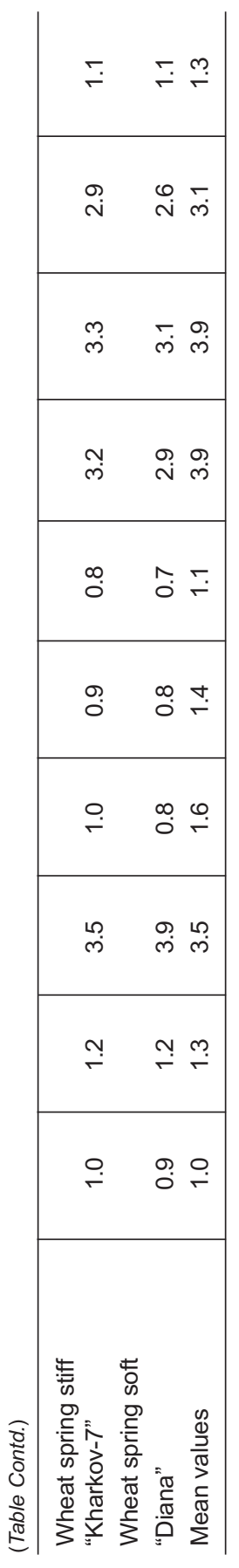




\section{Practical Spectroscopy in Agriculture and Food Science}

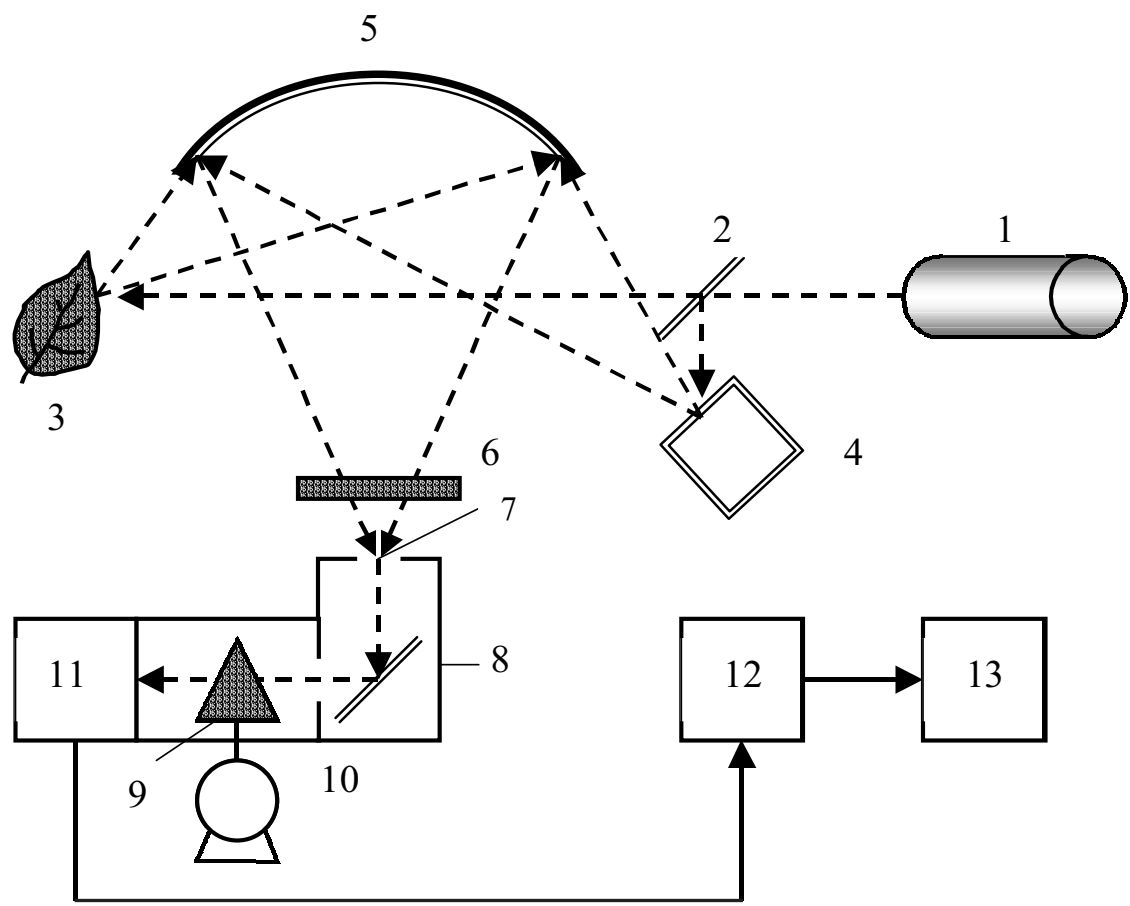

Fig. 8.1 Schematic representation of laser spectrofluorometer (explanation in text) [Posudin, 1998]

Wheat, rye, barley and hybrids have the following values: $K_{4}, K_{5} \approx 1-8$, $K_{6} \approx 1-6, K_{7}, K_{8} \approx 1-13, K_{9} \approx 3-9$, while the value of corn is one order higher than these ratios which can reach values of 11-27.

It was interesting to study these ratios for the different species of the same family (wheat). The results (Table 8.2) demonstrate the narrow interval of the values of such fluorescence indices as $K_{2} \approx 1.4-1.9, K_{3} \approx$ 3.3-4.6, and a wider interval for $K_{4} \approx 0.8-5.9$.

The growth of the plant from sprout to yellow stem is characterized by the decrease of mean values of fluorescence indices such as $K_{4} \approx 1.6$, $\mathcal{K}_{5} \approx 1.4, \mathcal{K}_{6} \approx 1.1, K_{7}, \mathcal{K}_{8} \approx 4, \mathcal{K}_{9} \approx 3$, while the values of $\mathcal{K}_{1} \approx 1$ and $\mathcal{K}_{3} \approx$ 3-4 remained constant (Table 8.3).

The dependence of these ratios on the length of stems (for wheat winter soft "Ivanovskaya-60" and wheat spring stiff "Svetlana") is also demonstrated in Table 8.3. The values of fluorescence indices $K_{1}, K_{2}, K_{3}$, $\mathcal{K}_{10}$ did not depend on the length of the stem, while the values of $\mathcal{K}_{4}$ and $K_{9}$ demonstrated a wider interval. 
In such a way, the fluorescence indices $\mathcal{K}_{4}=F(450) / F(690), K_{8}=$ $F(450) / F(735), K_{3}=F(690) / F(735), K_{2}=F(450) / F(530)$ are decreasing from etiolated to the green leaves. Such modifications of fluorescence spectra during greening of the leaves can be explained with difference of absorption spectra of etiolated and green leaves [Stober and Lichtenthaler, 1993]: the etiolated leaf demonstrates the broad absorption band of carotenoids near 425, 450 and $485 \mathrm{~nm}$, while the green leaf exhibits an absorption maximum at $430 \mathrm{~nm}$, a broad shoulder near 470 $500 \mathrm{~nm}$ and a maximum at $676 \mathrm{~nm}$.

The first stage of the dynamics of plant growth is characterized by the formation of etiolated sprouts which have a whitish-yellow colour at the top, bright-yellow in the middle part, and bluish-white at the base of the sprout. The etiolated (one to three days) sprouts demonstrate the intensive fluorescence in the blue part of the spectrum and weak fluorescence in the red part. The germinated seeds and etiolated sprouts have the identical shape of spectra; the seed spectra has a non-resolved blue band and a smaller red band. The etiolated leaves demonstrate the more intense blue fluorescence and the weakest red fluorescence at 690 $\mathrm{nm}$. The transformation of the etiolated sprout to yellow stem is accompanied by the decrease of the intensity of the blue-green fluorescence, increase of shoulder at $530 \mathrm{~nm}$, increase in the intensity of red band and appearance of maximum at $735 \mathrm{~nm}$; the most prolonged illumination induces the decrease of red fluorescence (Fig. 8.2).

The presence of only one maximum at $690 \mathrm{~nm}$ in fluorescence emission spectrum of etiolated leaves can be explained by a low (trace) amounts of chlorophyllide and/or chlorophyll $a$; the decrease in the intensity of fluorescence in the blue part of the spectrum can be provoked by a partial re-absorption of the emitted fluorescence by the photosynthetic pigments such as chlorophylls $a$ and $b$, and carotenoids; the disappearance of fluorescence in the green part of the spectrum can be explained by the re-absorption effects of chlorophyll $b$ and carotenoids [Lichtenthaler et al., 1981; Lichtenthaler, 1987; Stober and Lichtenthaler, 1992]. Appearance of maximum at $735 \mathrm{~nm}$ in the fluorescence spectrum of greening sprouts means that far-red chlorophyll fluorescence is slightly affected by the re-absorption processes [Stober and Lichtenthaler, 1993].

The same results were obtained by Stober et al. (1994). The blue-green fluorescence is a typical signature of yellowish etiolated leaves, white chlorophyll, and carotenoid-free white leaves. These leaves exhibited blue fluorescence 1.5 times stronger than the green leaf, and a distinct shoulder at $530 \mathrm{~nm}$ after illumination for two minutes with white light. The treatment of the leaves with the herbicide nonfluorazone (SAN 


\section{Practical Spectroscopy in Agriculture and Food Science}

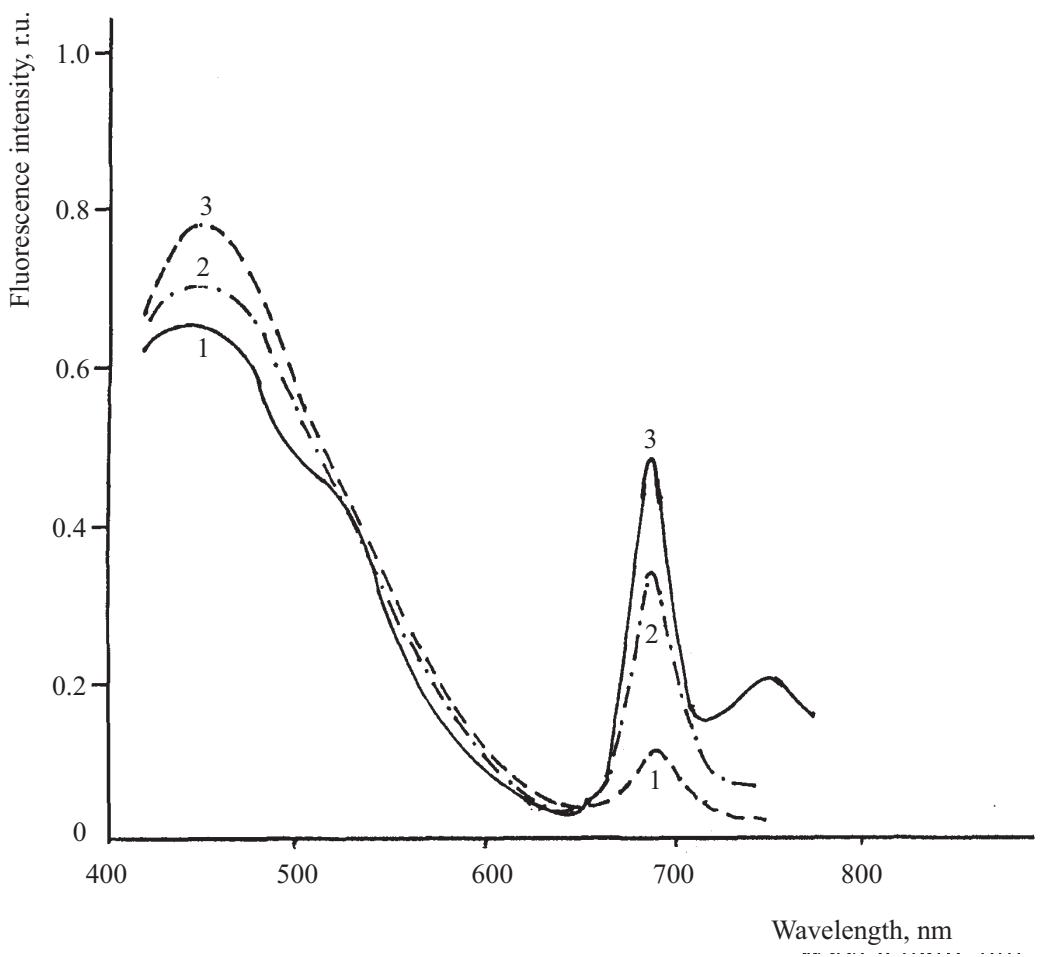

Fig. 8.2 Transformation of the fluorescence emission spectra from etiolated sprout to yellow stem: $1-1$ hour, $2-5$ hours, and $3-36$ hours of illumination with white light [Ivanitskaya and Posudin, 1989]

9789), which blocked the synthesis and accumulation of the carotenoids in wheat and bleached chlorophyll, leads to a fourfold increase of the blue fluorescence as compared to green leaves. A similar effect was obtained after acetone-washing of the leaves and removing of chlorophyll and carotenoids.

The dependence of fluorescence properties of the leaves on the phase of growth can be explained in the following way. It is known that the biosynthesis of chlorophyll $a$ in the leaves of the higher plants presents the complex process, which consists of the chain of photochemical transformations of chlorophyll from protochlorophyll. There are several dark and light stages of this process [Litvin and Belyaeva, 1971] which are presented in Fig. 8.3. The initial pigment form, protochlorophyll, participates in the photochemical reactions and undergoes a number of transformations, which are accompanied by a change of spectral properties (here the upper index corresponds to the fluorescence 
Spectroscopic Analysis of Agronomic Plants

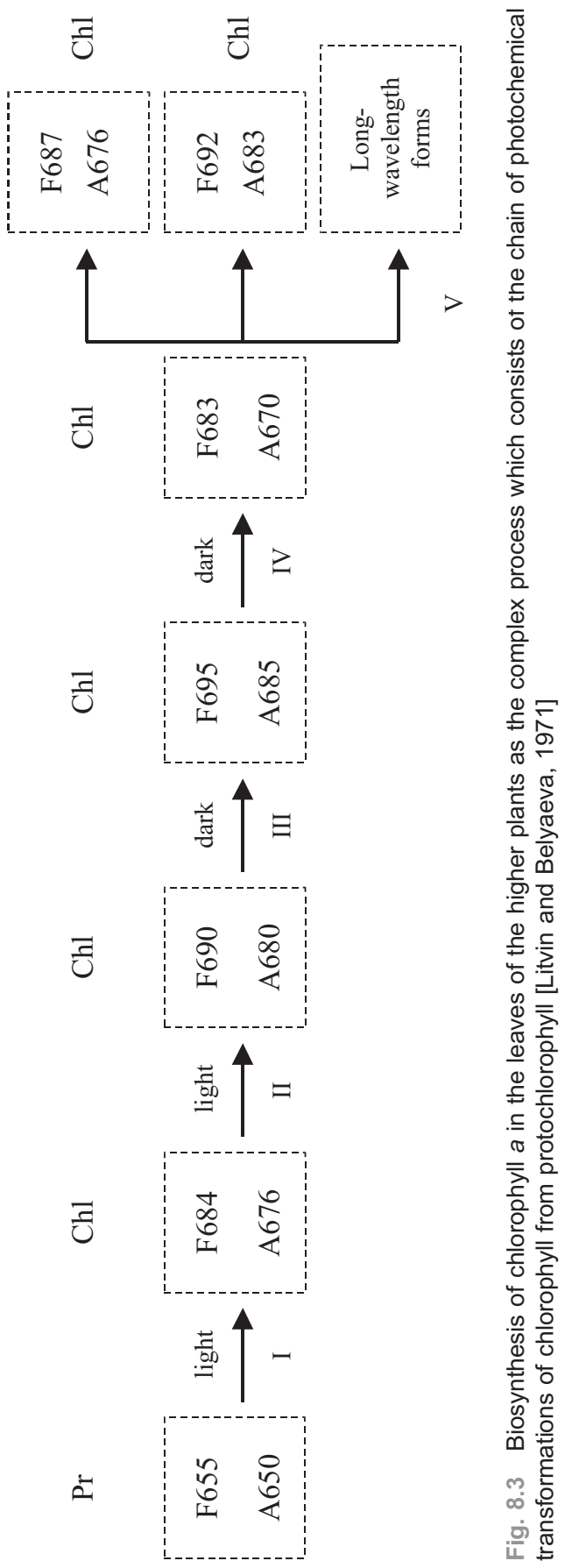




\section{Practical Spectroscopy in Agriculture and Food Science}

maximum, and the lower one to the absorption maximum). Hence, the first stage is the fast photoreaction of hydration and dark reorganization of pigment-protein complex of aggregated chlorophyll; the second stage presents the formation of phytol molecules and desegregation of pigment forms; the third stage is characterized by the accumulation of chlorophyll and increase in pigment concentration; the fourth stage is related to the appearance of the majority of pigment forms typical of the green leaf; and finally, the fifth stage demonstrates the consequent formation and accumulation of the long-wavelength forms of chlorophyll. All these stages are accompanied by corresponding spectral changes: the increasing intensities of red and far-red bands, and spectral shifts from 655 to $683-692 \mathrm{~nm}$ and to $722-738 \mathrm{~nm}$.

The fluorescence intensity depends on the part of sprout; the intensity of blue and red bands is minimal for the middle segment of the leaf and maximal for the upper and lower segments (Figs. 8.4-8.6).

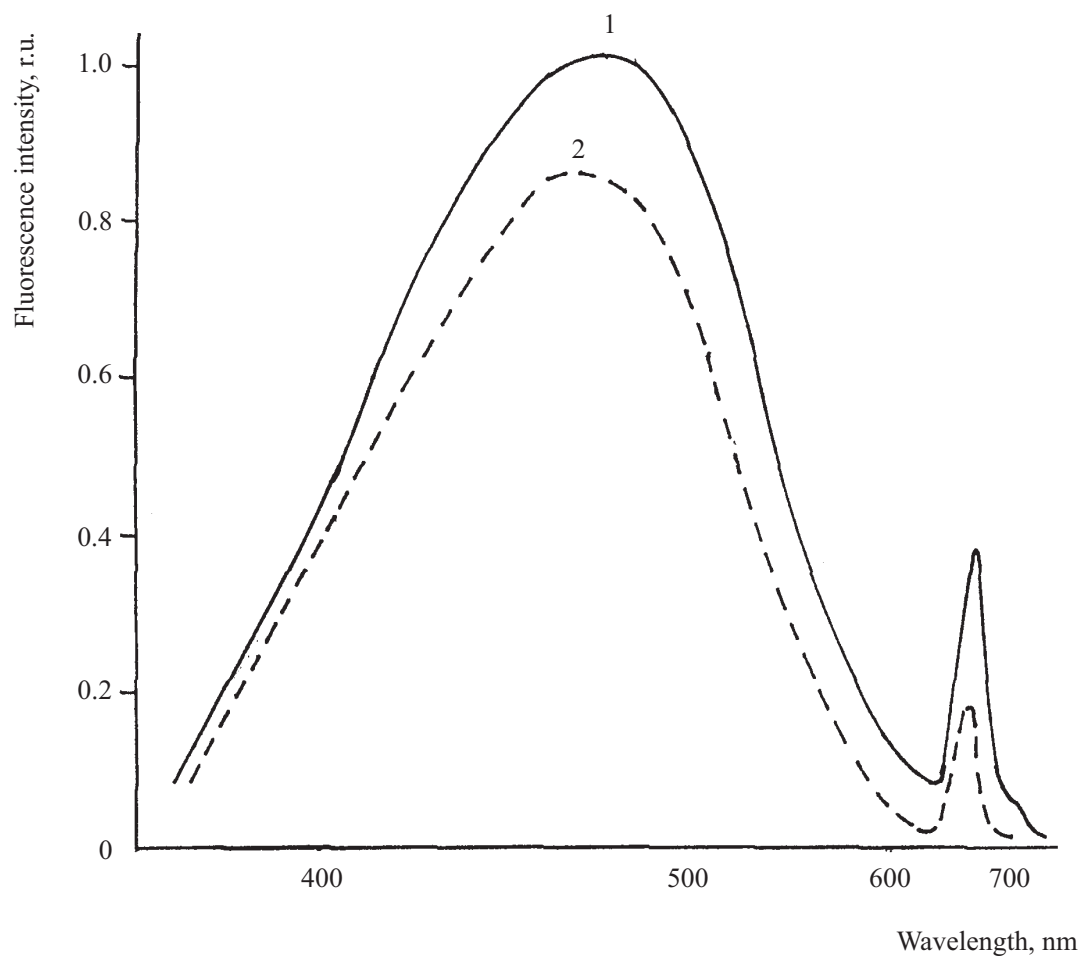

Fig. 8.4 Dependence of fluorescence intensity of winter stiff wheat "Korund" on parts of the leaf: 1 - lower segment; 2 - middle segment [Ivanitskaya and Posudin, 1989] 


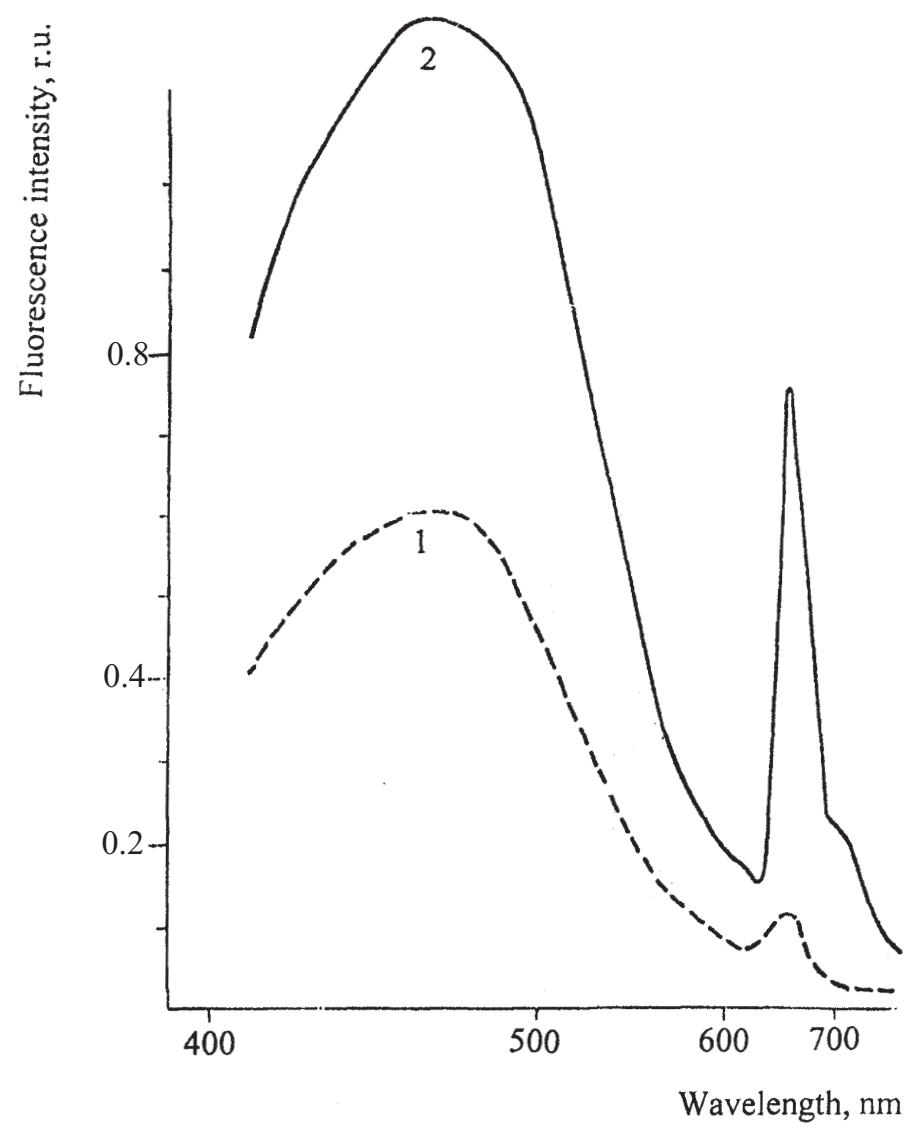

Fig. 8.5 Dependence of fluorescence intensity of rye "Zhitomyrskaya-tetra" on parts of the leaf: 1 - upper segment; 2 - middle segment [Ivanitskaya and Posudin, 1989]

\subsubsection{Effect of Pre-illumination}

The effect of pre-illumination of the etiolated stems of winter soft wheat ("Ivanovskaya-60" and "Mironovskaya-808") by white light (4000 lx during five hours) is presented in Table 8.4. There is a certain decrease in the fluorescence indices such as $K_{3}-K_{4}$.

Longer illumination (during 36 hours) of spring wheat provokes the greening of stems; the fluorescence indices $\mathcal{K}_{3}-\mathcal{K}_{4}$ decrease (Fig. 8.7) after irradiation (Table 8.5).

Such a behaviour of the fluorescence spectra of etiolated leaves during greening correlates with the total chlorophyll content and can be explained by partial re-absorption of the emitted fluorescence by the 


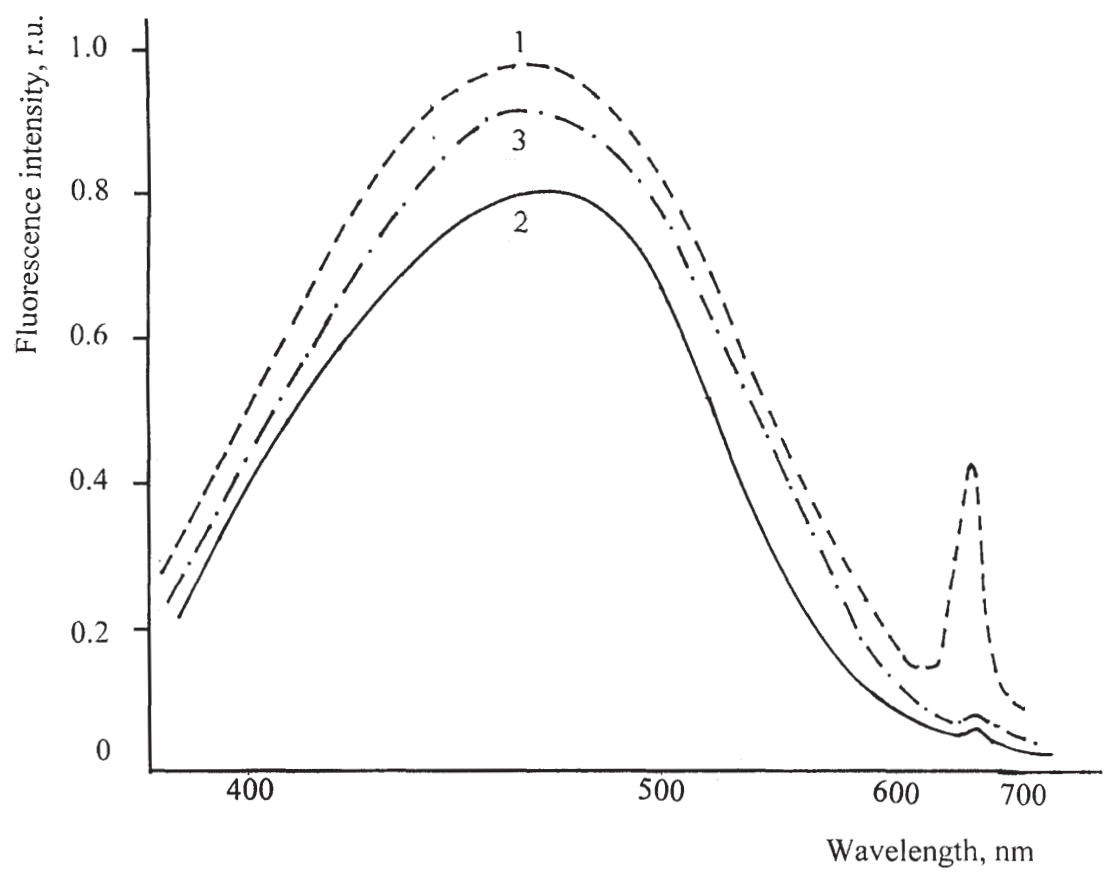

Fig. 8.6 Dependence of fluorescence intensity of hybrid amphidiploid "Diploid 3/5" on parts of the leaf: 1 - upper segment; 2 - middle segment; 3 - lower segment [lvanitskaya and Posudin, 1989]

photosynthetic pigments [Stober and Lichtenthaler, 1992; Lichtenthaler et al., 1993].

\subsubsection{Fluorescence Emission Spectra of Vegetables}

It is very informative to find the specific spectral features of different plant species; such spectral "fingerprints" can be used in the remote sensing vegetation for identification and inventory of agricultural fields.

The fluorescence characteristics of leaves of the following vegetables have been investigated: potato, cucumber, tomato, bean, vegetable marrow, pumpkin, pepper, haricot and horse-radish (Table 8.6).

The most prominent difference in the fluorescence spectra is obtained accurately for vegetables; the species demonstrate the different fluorescence intensities in blue, red and far-red parts of the spectrum (Figs. 8.8-8.16). 
Spectroscopic Analysis of Agronomic Plants

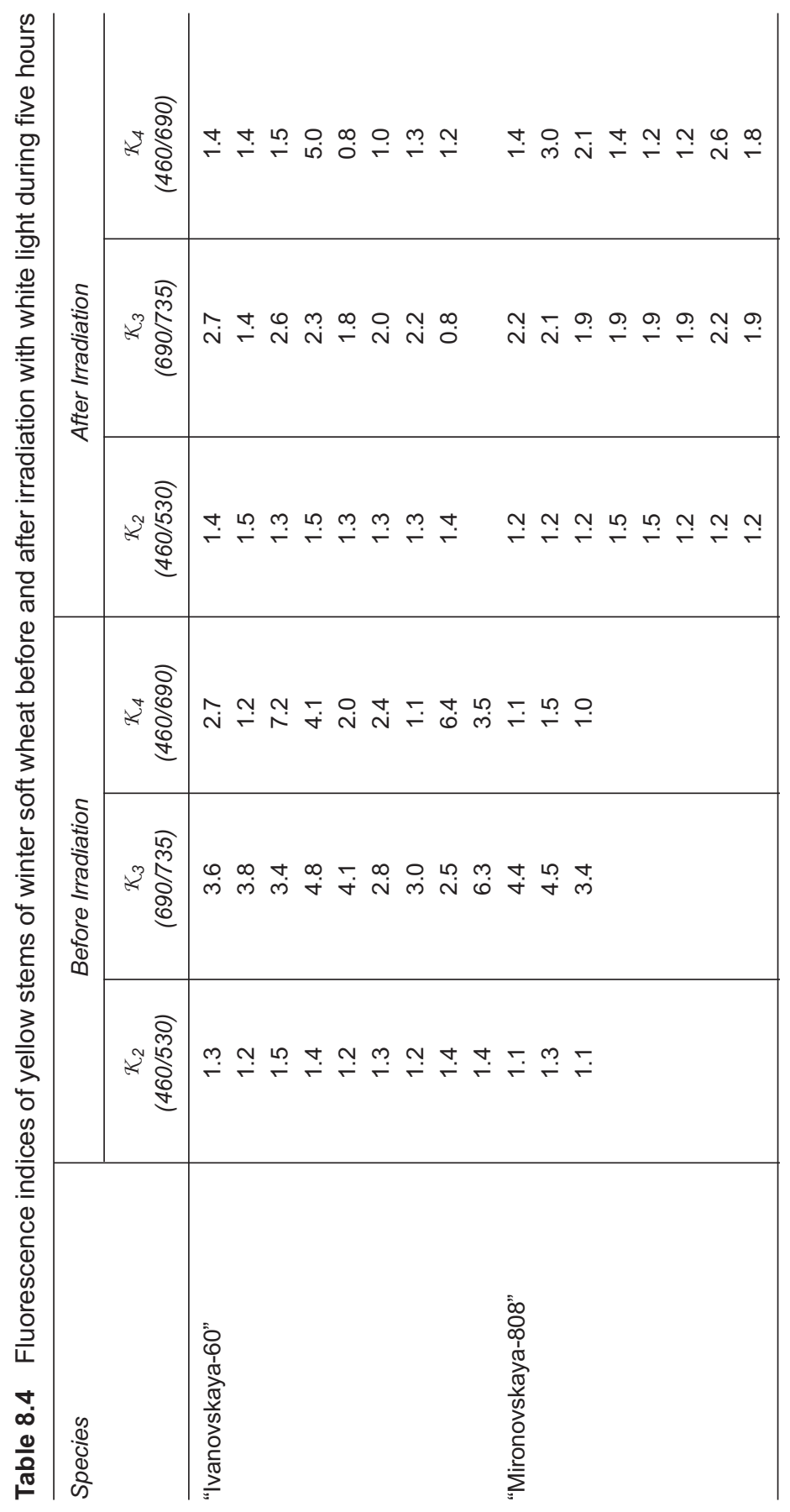




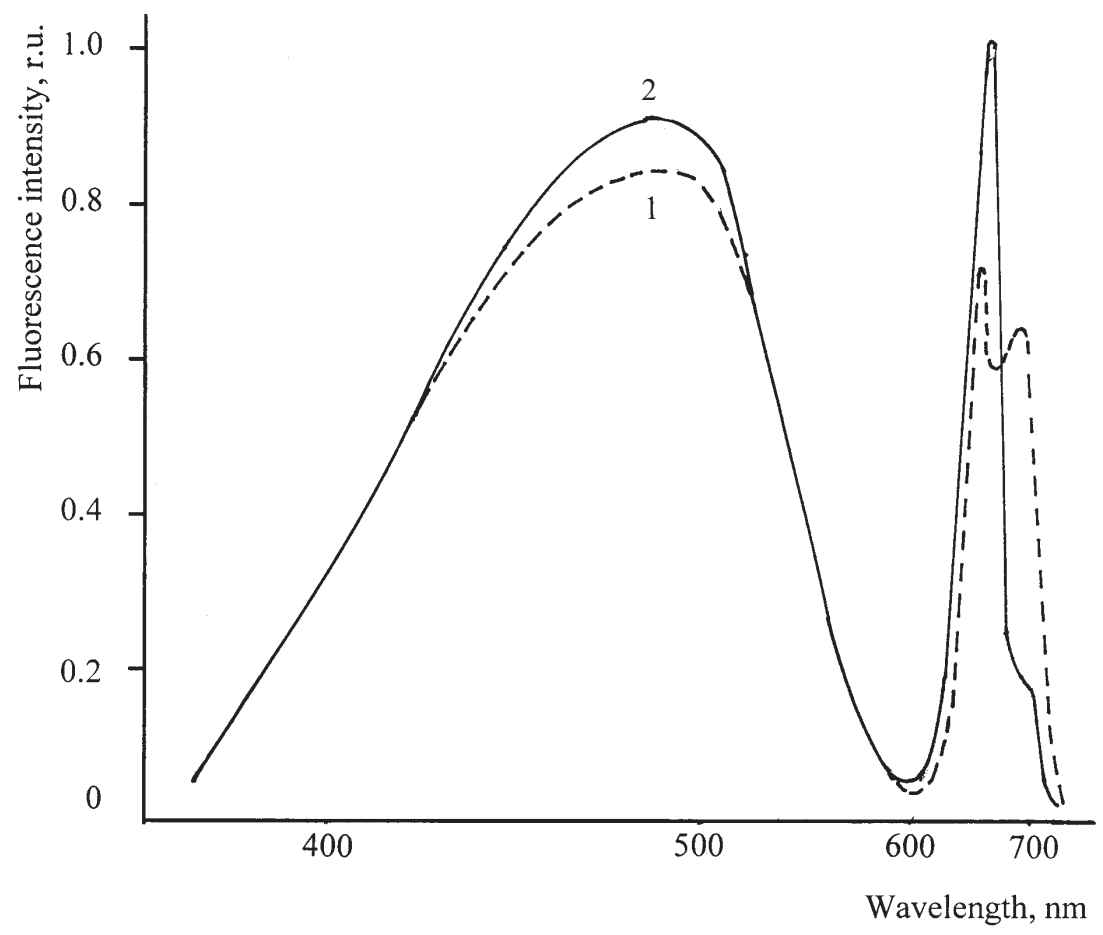

Fig. 8.7 Dependence of fluorescence intensity of wheat winter stiff "Svetlana" on the illumination of the sample during 36 hours with white light $(4,000 \mathrm{~lx}): 1$ - before, 2 - after illumination [Ivanitskaya and Posudin, 1989]

\subsubsection{Effect of the Leaf Side}

An overwhelming majority of the agronomic plants have bifacial leaves: the upper (ventral) side of the leaf includes palisade parenchyma with densely packed cells; the lower (dorsal) side contains spongy parenchyma with less quantity of cells. Such leaf morphology is typical for $\mathrm{C}_{3}$-plants. In that way the upper side of the leaf presents a higher concentration of chlorophyll than the lower one. It was shown [Lichtenthaler and Rinderle, 1988] that when the fluorescence is excited and sensed from the upper leaf side, the maxima of emission at 690 and $735 \mathrm{~nm}$ are approximately equal in height; the value of the maximum at $690 \mathrm{~nm}$ is higher than the value of the maximum at $735 \mathrm{~nm}$ during excitation of fluorescence from the lower side of the leaf.

The $\mathrm{C}_{4}$-plants are characterized by the so-called Kranz-anatomy - the concentric disposition of mesophyll cell layers; the difference in the 
Spectroscopic Analysis of Agronomic Plants

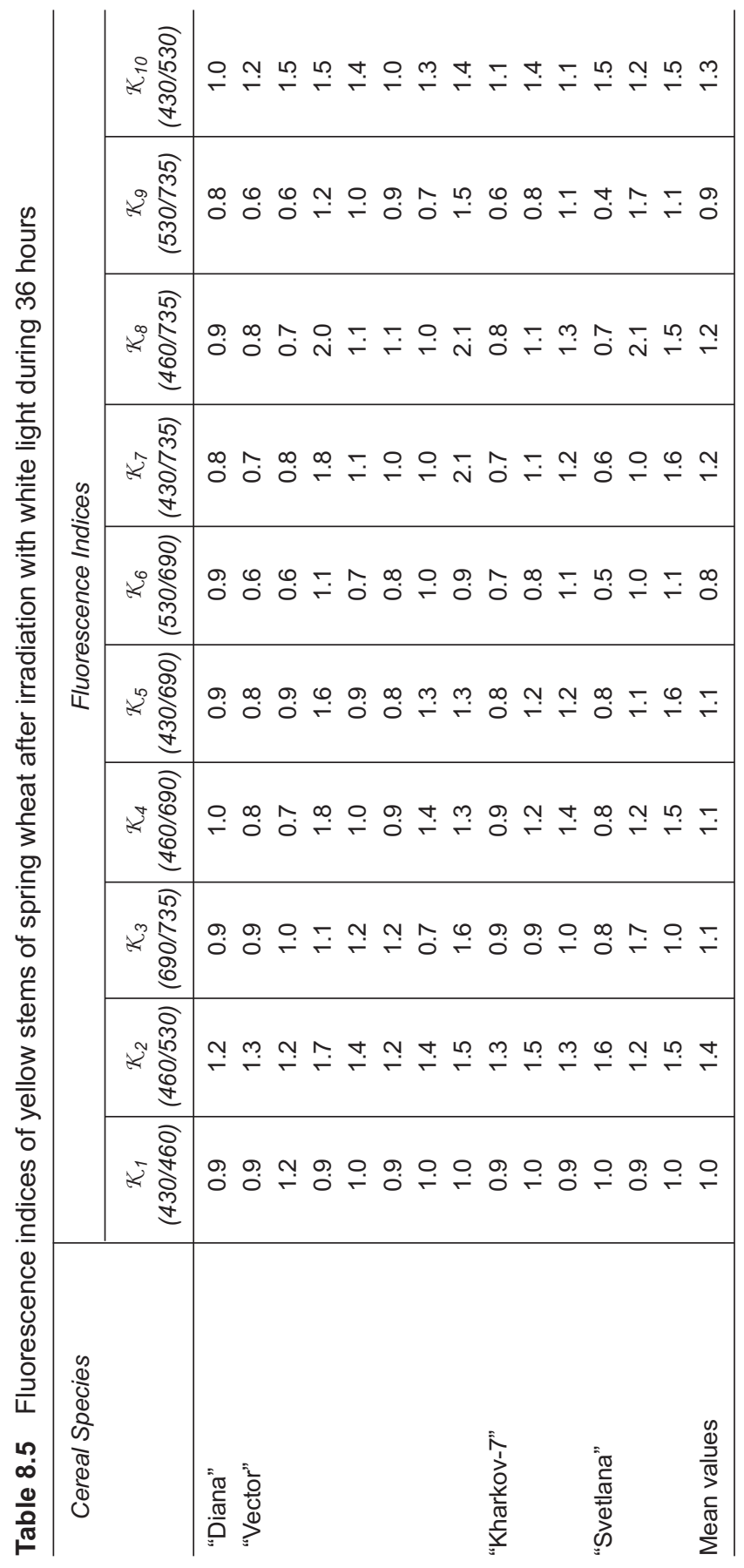




\section{Practical Spectroscopy in Agriculture and Food Science}

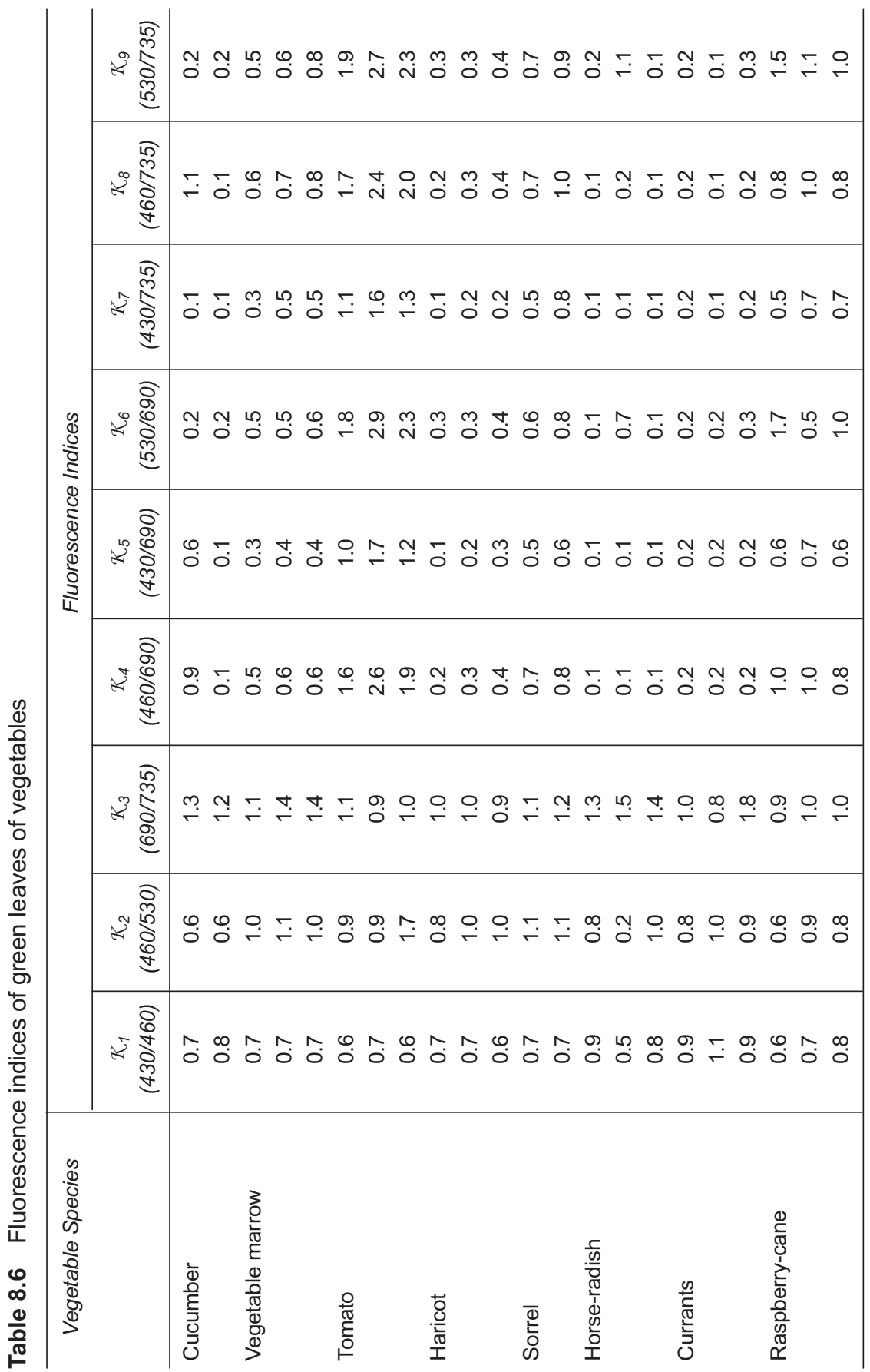




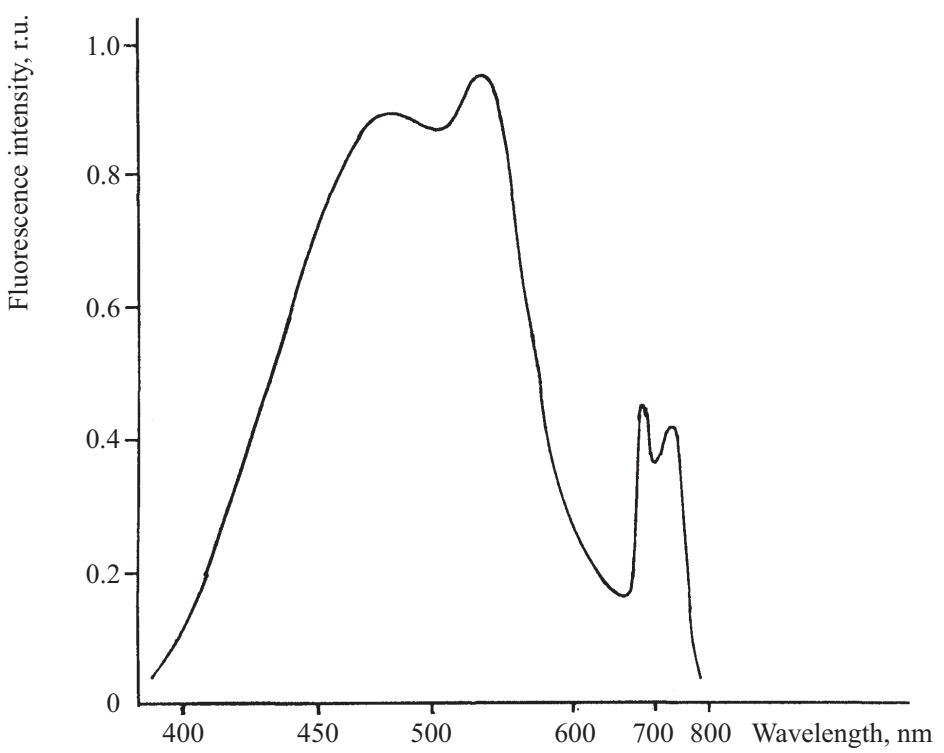

Fig. 8.8 Fluorescence emission spectrum of tomato [Ivanitskaya and Posudin, 1989]

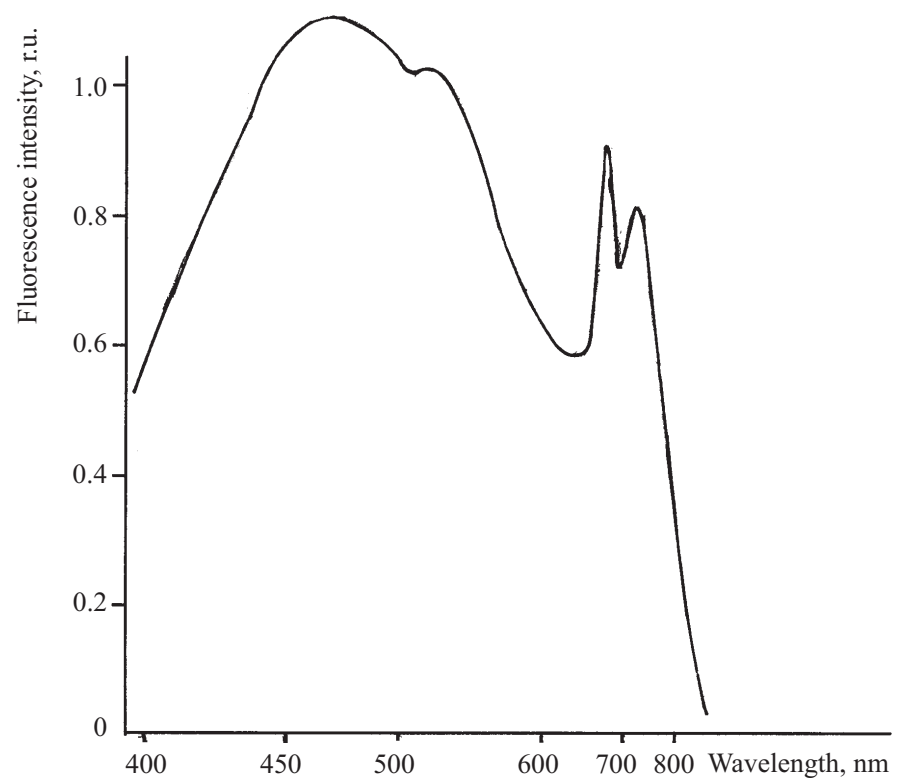

Fig. 8.9 Fluorescence emission spectrum of potato [Ivanitskaya and Posudin, 1989] 


\section{Practical Spectroscopy in Agriculture and Food Science}

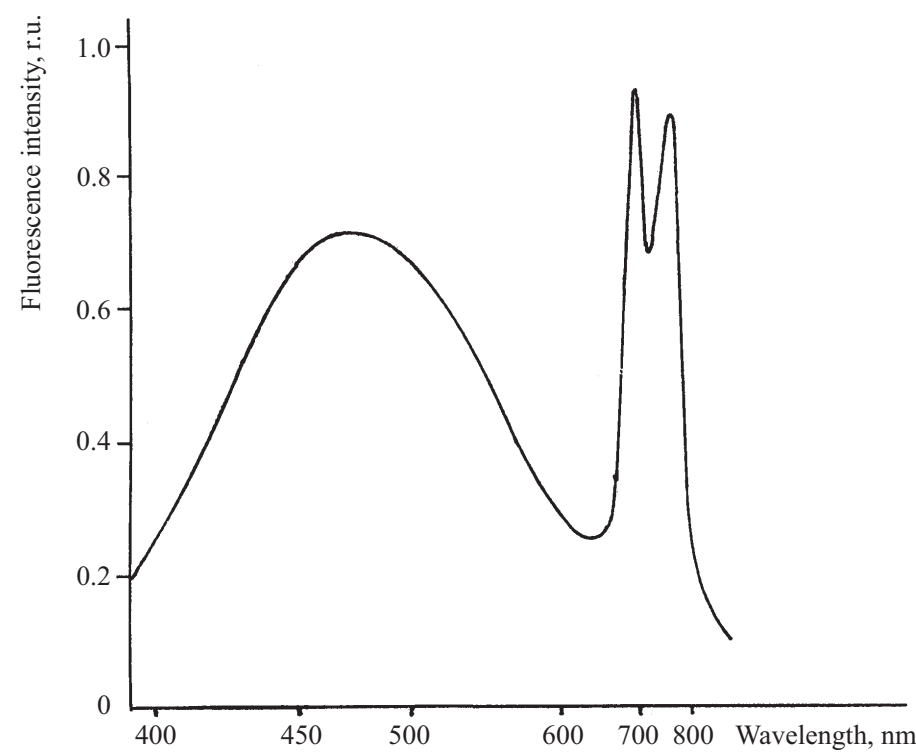

Fig. 8.10 Fluorescence emission spectrum of pumpkin [Ivanitskaya and Posudin, 1989]

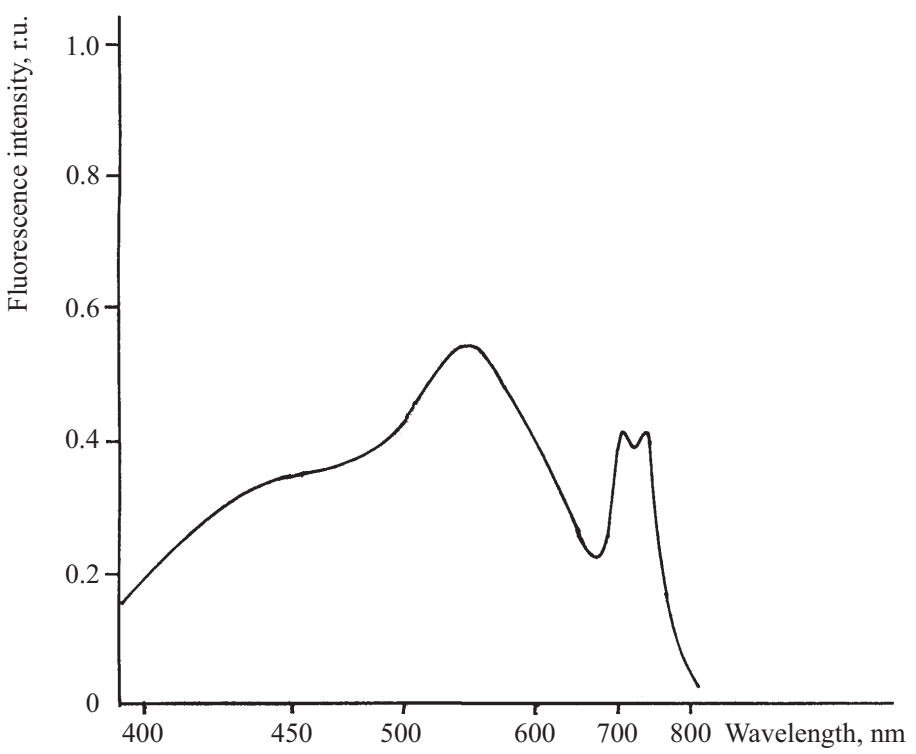

Fig. 8.11 Fluorescence emission spectrum of pepper [Ivanitskaya and Posudin, 1989] 


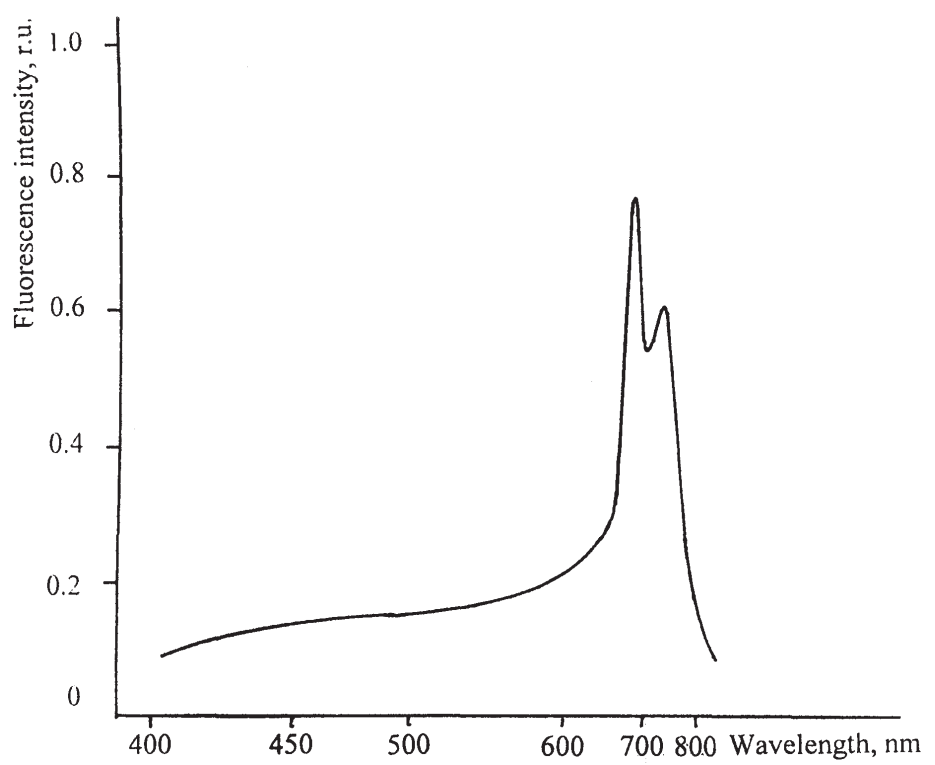

Fig. 8.12 Fluorescence emission spectrum of horse-radish [Ivanitskaya and Posudin, 1989]

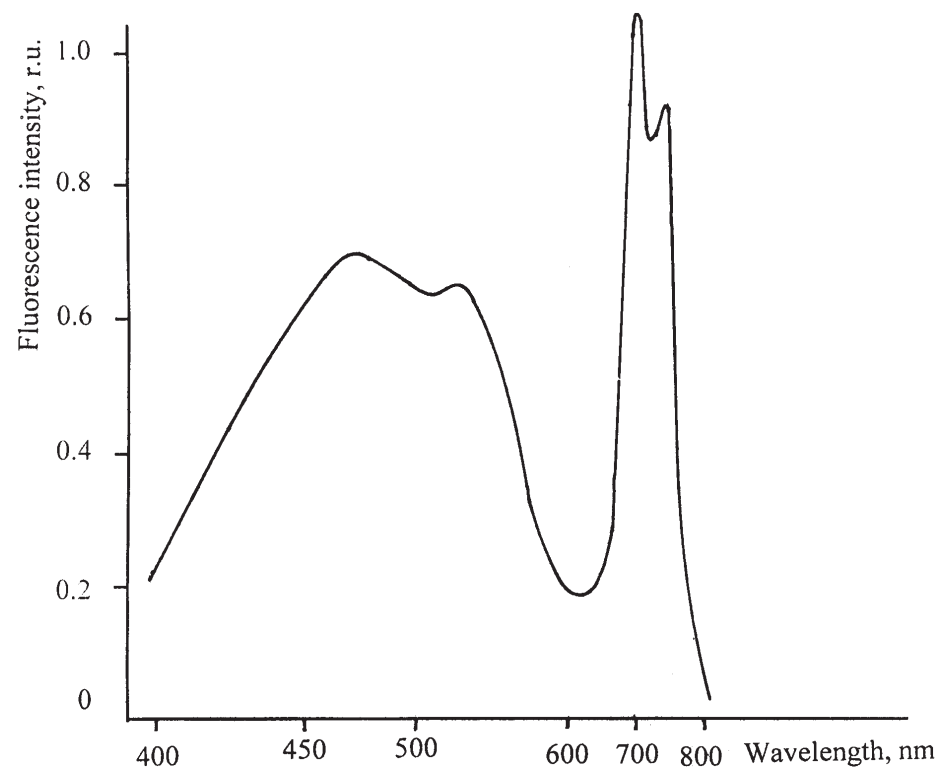

Fig. 8.13 Fluorescence emission spectrum of sorrel [Ivanitskaya and Posudin, 1989] 


\section{Practical Spectroscopy in Agriculture and Food Science}

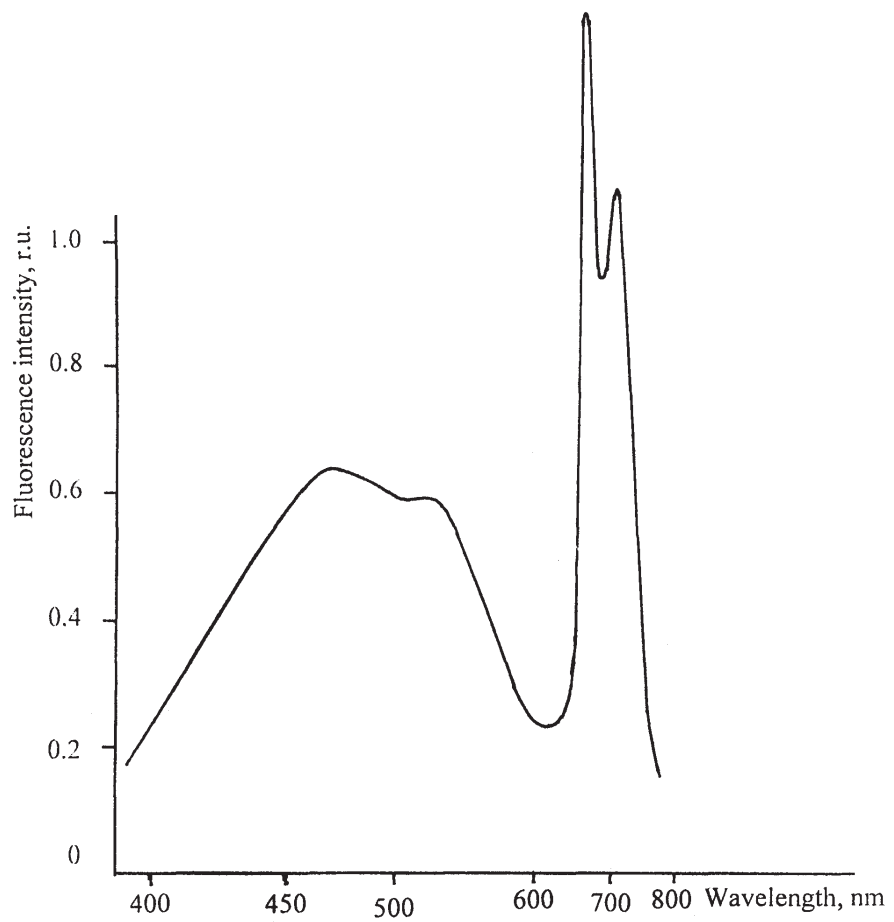

Fig. 8.14 Fluorescence emission spectrum of vegetable marrow [Ivanitskaya and Posudin, 1989]

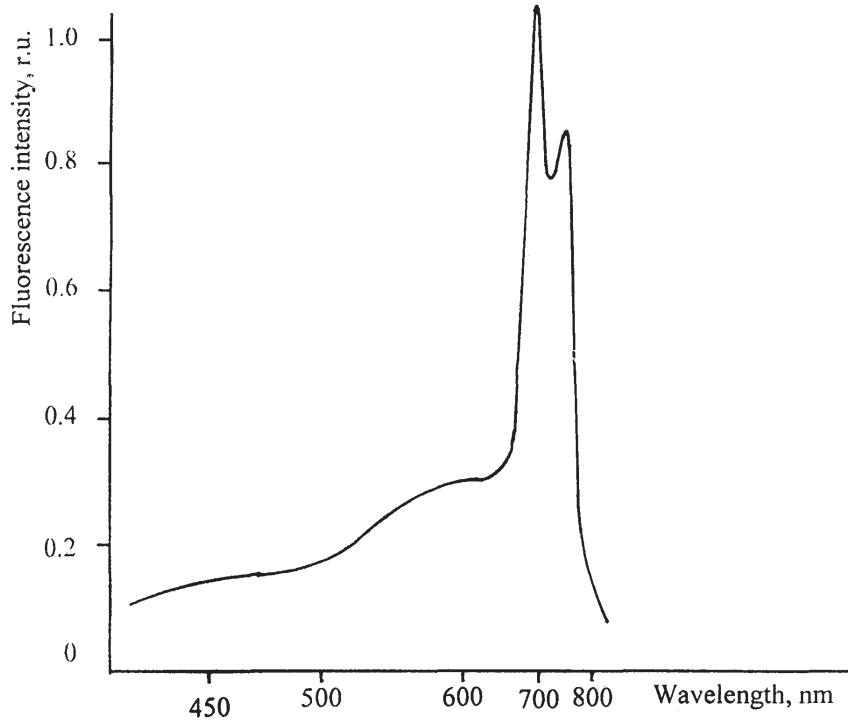

Fig. 8.15 Fluorescence emission spectrum of cucumber [Ivanitskaya and Posudin, 1989] 


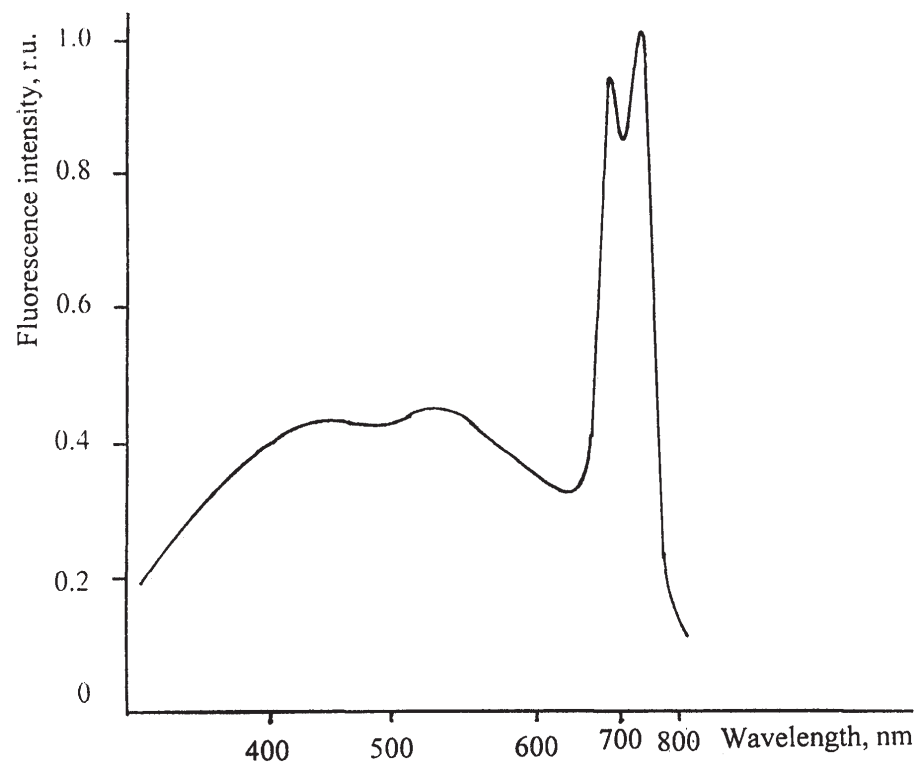

Fig. 8.16 Fluorescence emission spectrum of haricot [Ivanitskaya and Posudin, 1989]

fluorescence spectra of such equifacial leaves (for example, the leaves of maize) between the upper and lower side is rather small. The authors consider that if the concentration of chlorophyll is higher in the upper side, the probability of re-absorption of the emitted fluorescence is also higher; correspondingly, the values of the ratio F690/F735 are: 0.85-1.10 for upper side and 1.20-1.70 for the lower side. Chappelle et al. $(1984 a, b)$ established that laser-induced fluorescence of the back side of the soybean leaf is greater than that of the front side. These fluorescence distinctions are related to the differences either in morphological structure, or in the photosynthetic properties of both sides.

The difference in the fluorescence spectra between upper and lower leaf sides is also demonstrated by several $\mathrm{C}_{4}$ plants such as maize (Fig. 8.17).

\subsubsection{Effect of Leaf Nodal Position and Age}

The effect of nodal position of leaves of the vegetables is presented in Table 8.7. This effect of the leaf position on the plant stem on the fluorescence characteristics can be related to the age of the leaf (the youngest dark green leaves are located in the upper nodes) and to the different levels of photosynthetic activity. The older, yellow-green leaves 


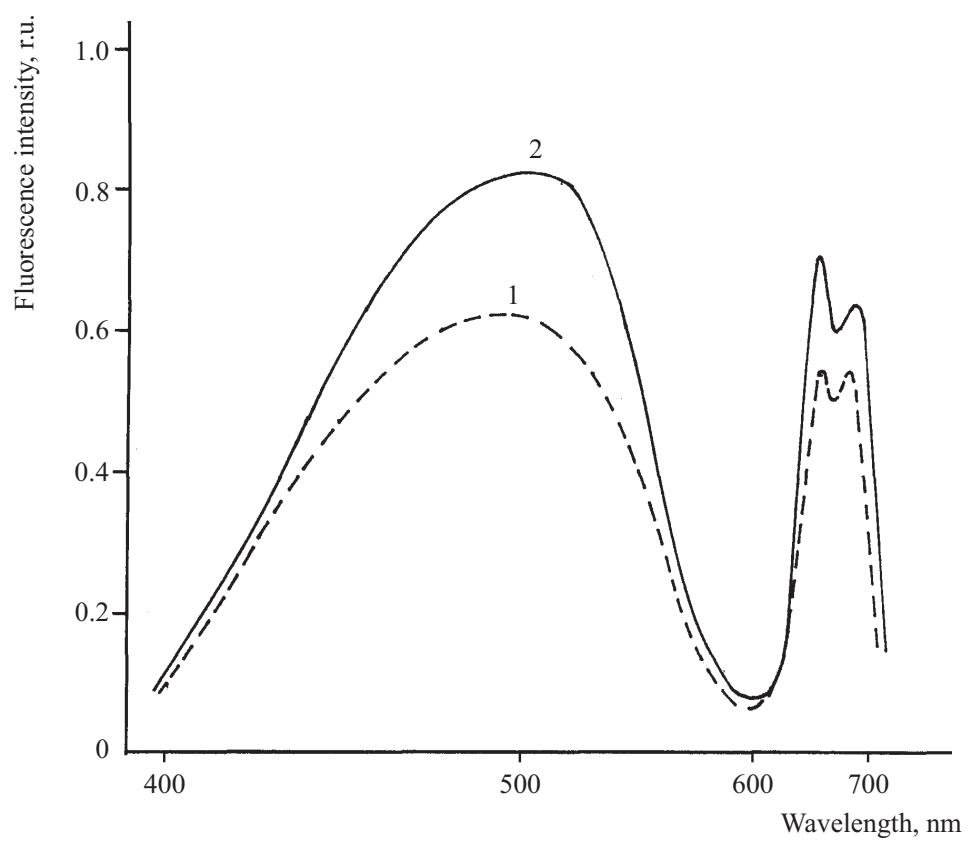

Fig. 8.17 Fluorescence emission spectra of upper (1) and lower (2) sides of maize representative of $\mathrm{C}_{4}$ plants [Ivanitskaya and Posudin, 1989]

of potato demonstrate lower intensities of fluorescence than the younger dark green leaves (Fig. 8.18). These results are in complete agreement with those observed by Lang et al. (1992), i.e. decrease of the blue fluorescence emission from young to an old leaf. The reason is the same - different concentrations of chlorophyll during leaf development and correspondingly different levels of re-absorption of fluorescence.

\subsubsection{Effect of Fertilization}

The effect of different natural and artificial fertilizers, such as manure, $N_{30} P_{30} K_{30}$ and $N_{15} P_{15} K_{15}$, and "biohumus" (product of worm activity in the soil), just as the combinations of these fertilizers, on the fluorescence of tomato leaves (sort "Kechkemeti 549") was observed by the author and his colleagues [Gorodny et al., 1992]. It was shown that the fluorescence parameters could be used for estimation of fertilization efficiency.

\subsubsection{Effect of Plant Diseases}

It was very interesting to establish the effect of plant pathology on the chlorophyll fluorescence of leaves. Bean plants with affected root system [Posudin et al., 1992] have been used in this experiment. The level of the 
Spectroscopic Analysis of Agronomic Plants

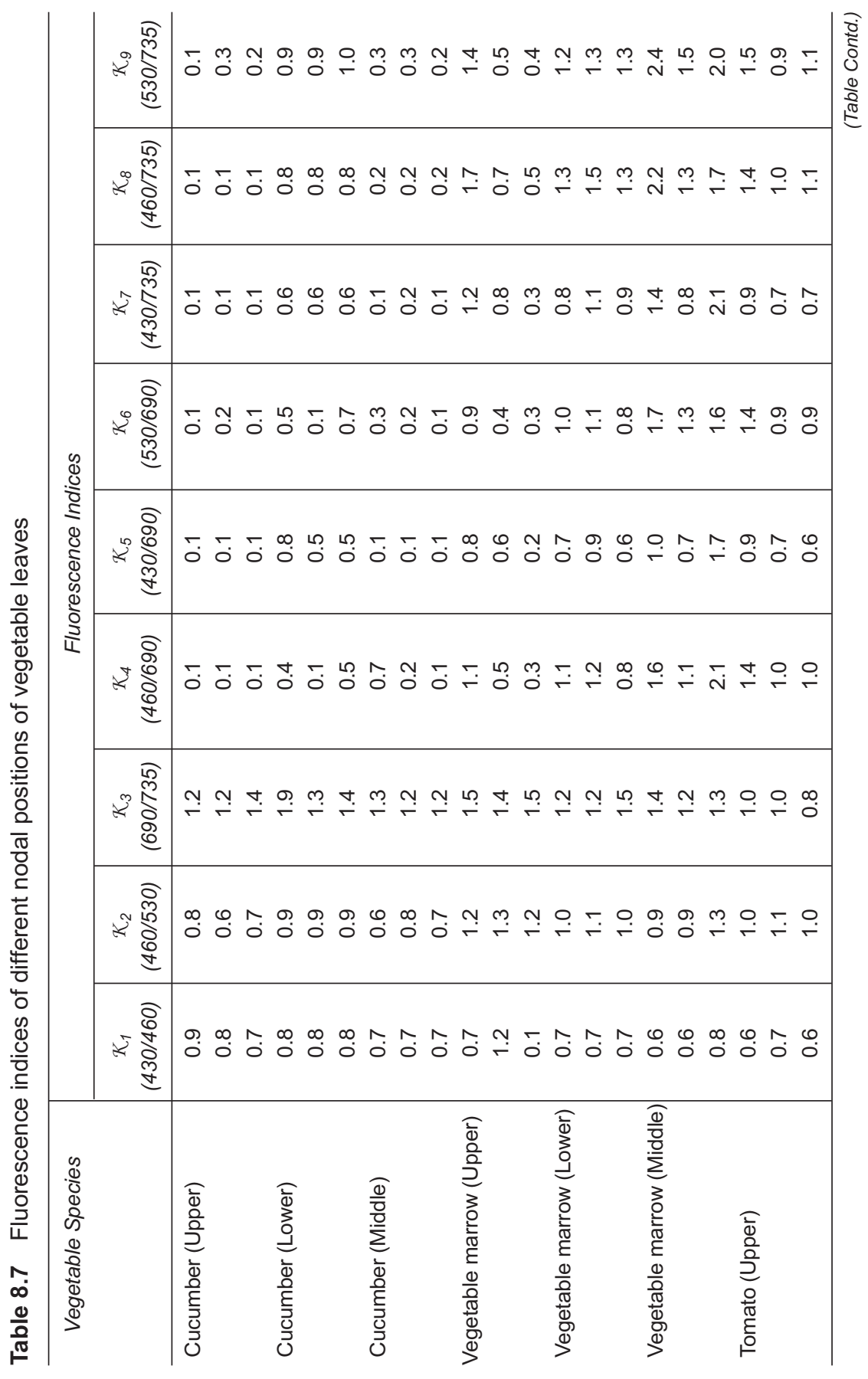




\section{Practical Spectroscopy in Agriculture and Food Science}

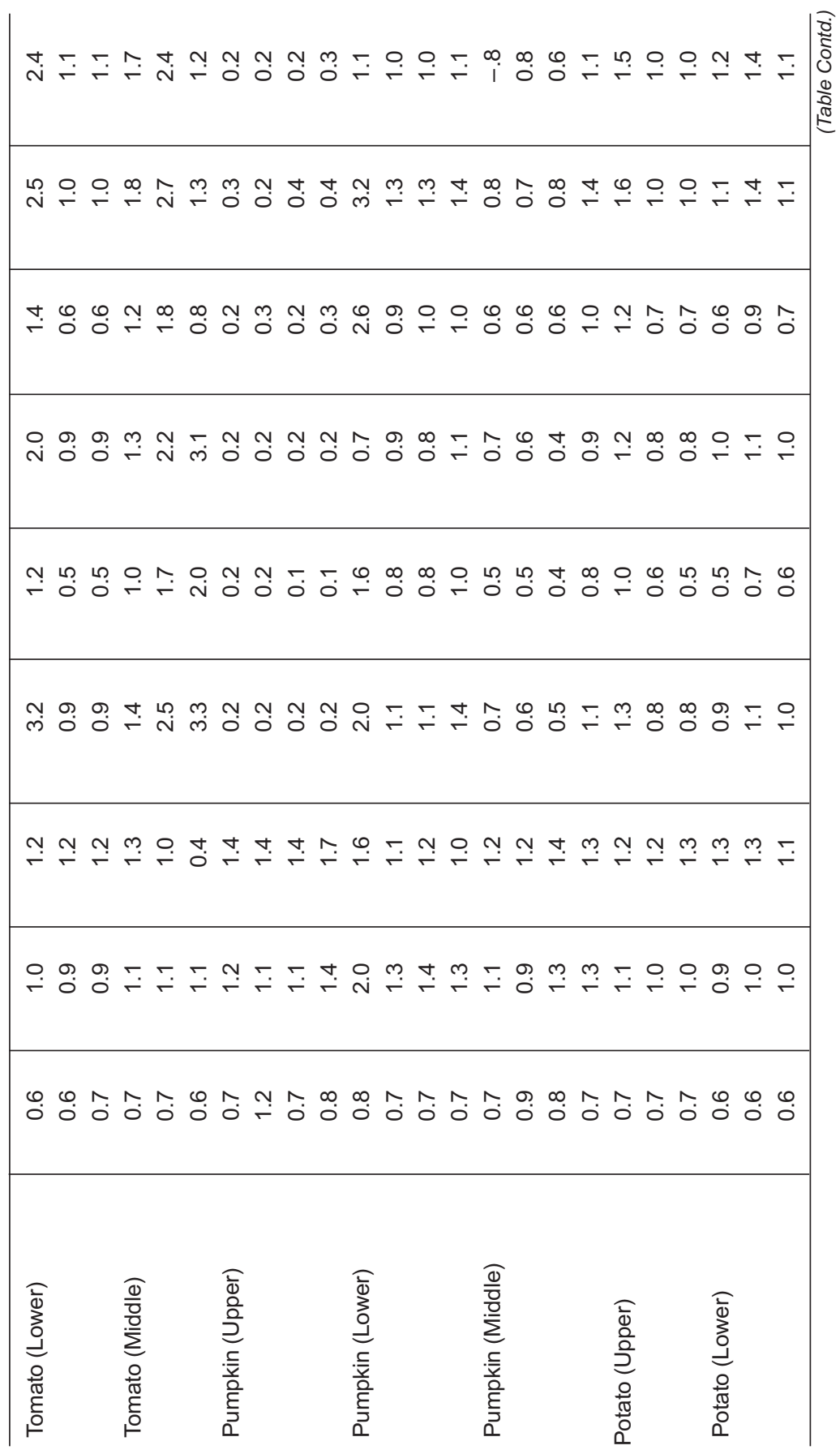




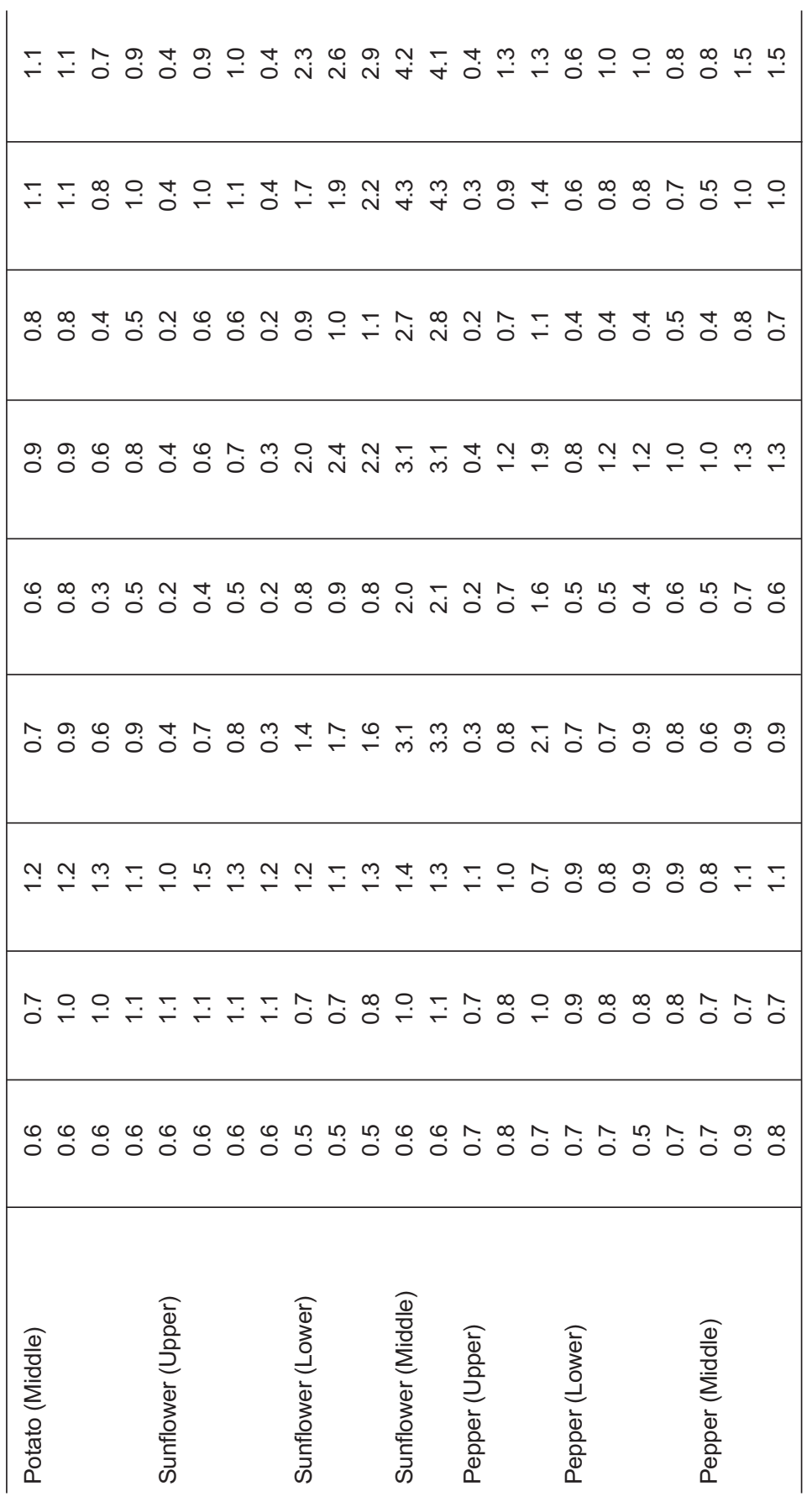




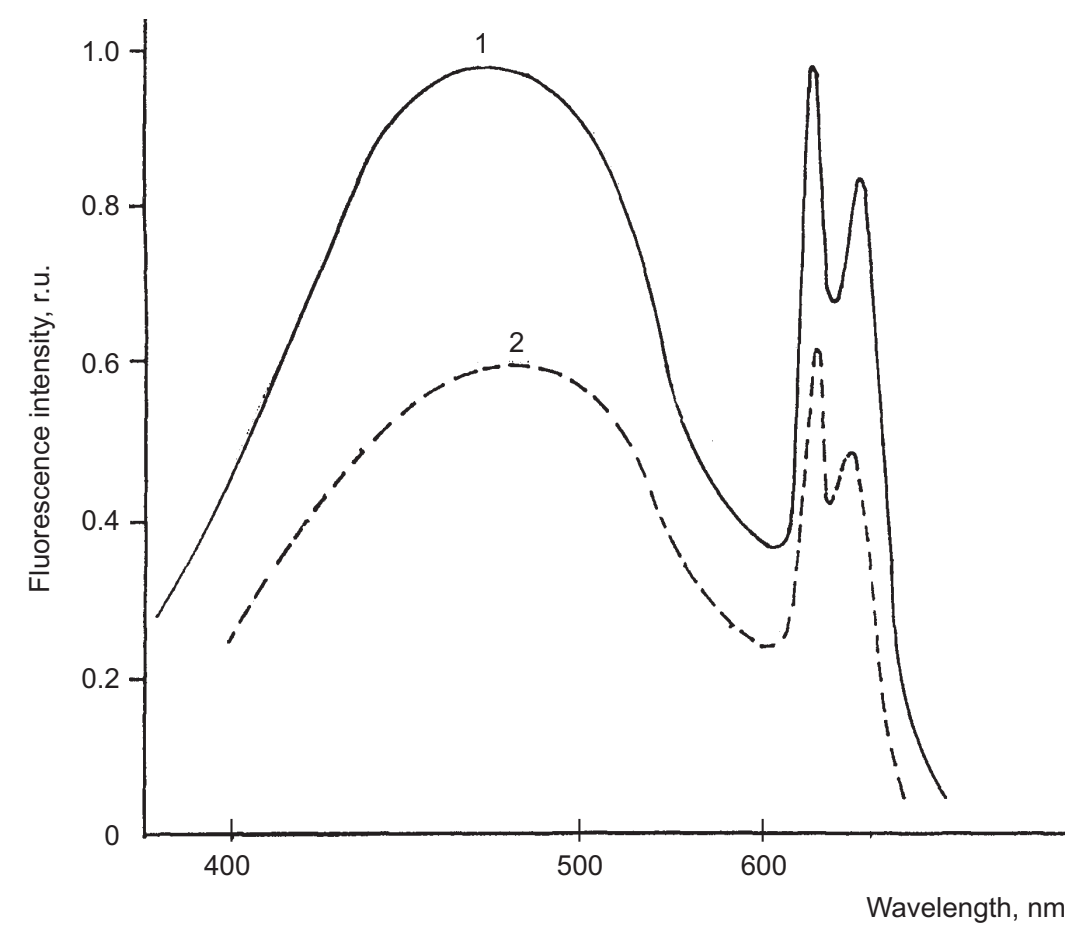

Fig. 8.18 Fluorescence emission spectra of leaves of potato: 1 - older yellow-green; 2 younger dark green [Ivanitskaya and Posudin, 1989]

lamage, injury was estimated quantitatively due to 5 -ball system $(0=$ healthy roots, 1 = small, 2 = middle, 3 = strong, $4=$ utter defeat of the roots). The typical dependence of fluorescence intensity of the leaf on the level of the root defeat for different parts of leaf is presented in Fig. 8.19. Probably, fluorescence parameters can be applied as diagnostic criteria in the science of plant protection.

\subsection{FLUORESCENCE INDUCTION KINETICS}

\subsubsection{Theory of Fluorescence Induction Kinetics}

If a green photosynthetically active leaf is adapted to darkness during a certain period (15-20 minutes) of time, the cooperation of photosystems PSI and PSII is disturbed. The fact is that the illumination of the darkadapted leaf provokes the so-called fluorescence induction (Kautsky effect) [Kautsky and Hirsch, 1931]. It is possible to distinguish two parts of the temporal behaviour of chlorophyll fluorescence: fast increase of 


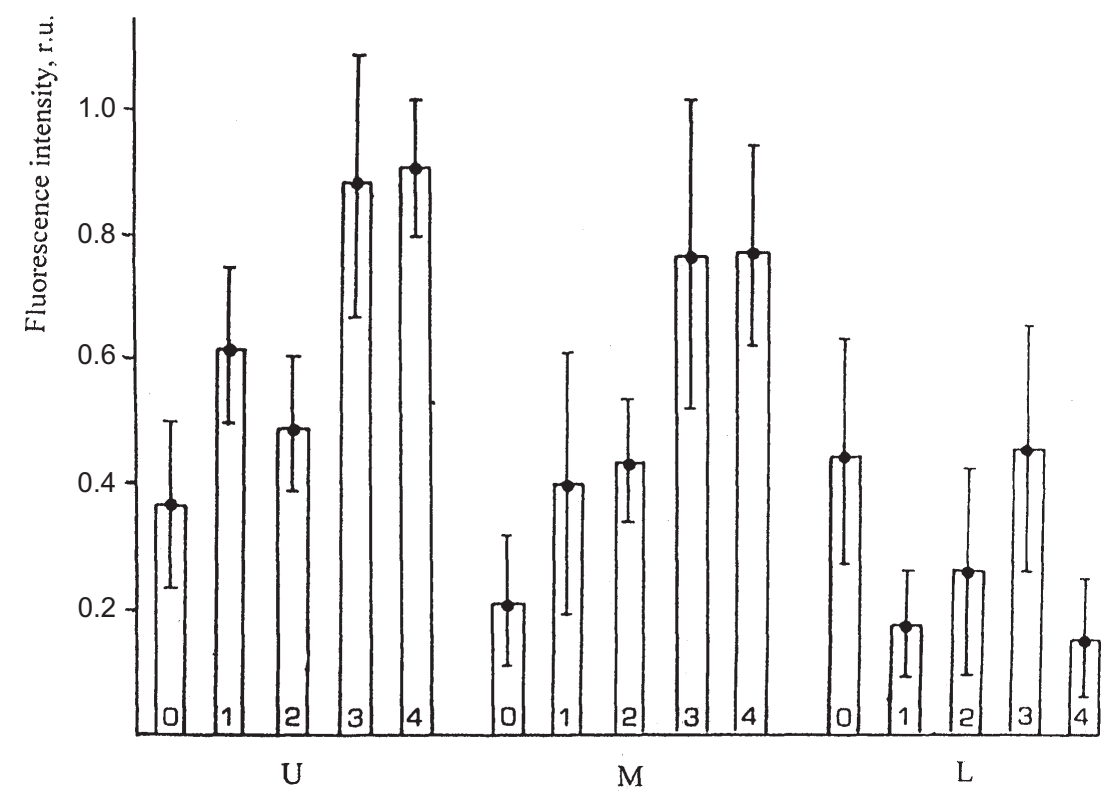

Fig. 8.19 Typical dependence of fluorescence intensity of the leaf on the level of the root $\equiv$ amage, injury which was estimated with different (from 0 to 4 ) points and from parts of leaf $\square$ J - upper, M - middle, L - lower) [Posudin et al., 1992]

fluorescence intensity up to maximal value $f_{m}$ during $100-500 \mathrm{~ms}$, and slow decrease of fluorescence up to stationary level $f_{s}$. The photosynthetic apparatus of the dark-adapted leaf is in state I, while the illumination induces its transfer to state II. A typical fluorescence induction curve is presented in Fig. 8.20.

\subsubsection{Plant Samples}

It is informative to provide the comparative analysis of fluorescence characteristics of different varieties of the same specie of the plants. The objective of this investigation is to study the changes in chlorophyll fluorescence during leaf development and under stress conditions of two varieties of green pea Pisum sativum, and to use chlorophyll fluorescence parameters as indices of plant status and taxonomic criteria. Two varieties of green pea Pisum sativum, Determinantny and Bakara, from the collection of National Agricultural University of Ukraine (Kiev), were used in these experiments. The plants were grown in the green-house of Botanical Garden of the University of Karlsruhe (Germany) at an ambient temperature of $22^{\circ} \mathrm{C} \pm 4^{\circ}$ of on a peat containing mineral nutrients (TKS II). Pieces of $9 \mathrm{~mm}$ diameter were cut off from the pea 


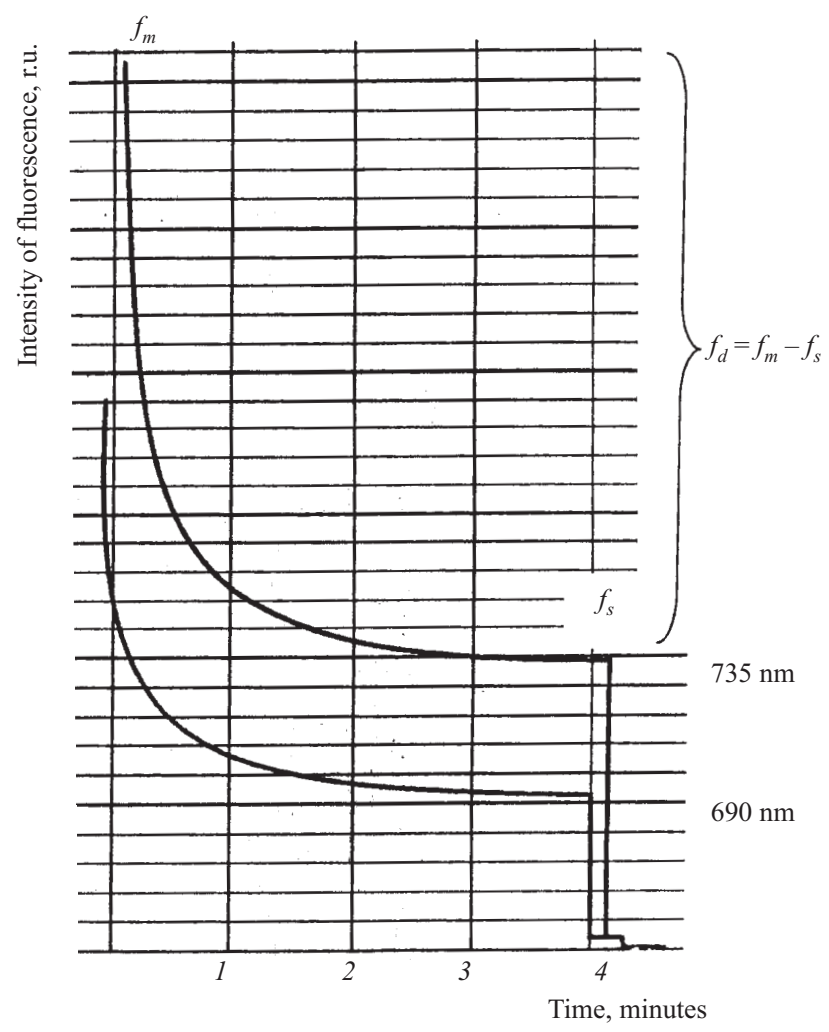

Fig. 8.20 Chlorophyll-fluorescence induction curves of 15-min predarkened leaves of green pea Pisum sativum recorded in the $690 \mathrm{~nm}$ region (lower curve) and $735 \mathrm{~nm}$ region (upper curve) [Posudin, 1997, 2002]

leaves and kept in darkness for 15 minutes before the chlorophyll fluorescence measurements.

\subsection{INSTRUMENTATION}

A principal set-up for measuring parameters of fluorescence induction is presented in Fig. 8.21. Two-wavelength fluorometer (National Agricultural University, Kiev, Ukraine) was used for illumination of the predarkened leaves and control of fluorescence emission at two wavelengths at 690 and $735 \mathrm{~nm}$. It consists of mercurial lamp 1 DRK-120 of superhigh pressure which is used as the source of fluorescence excitation. The chlorophyll fluorescence kinetics of dark adapted pea leaves was analyzed during five minutes. The radiation of the lamp 1 


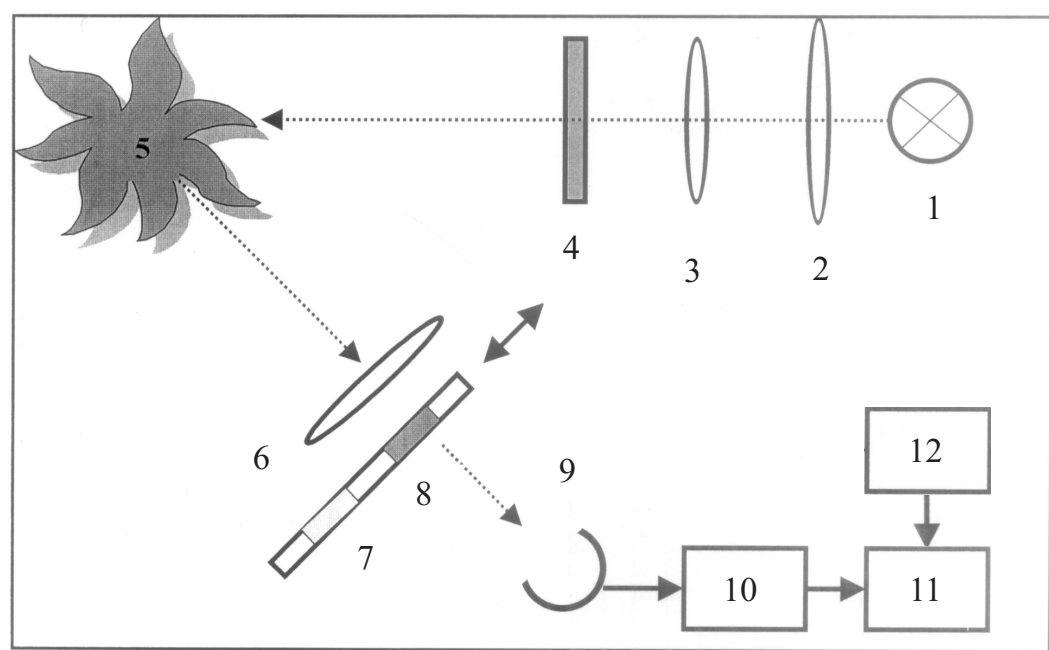

Fig. 8.21 Two-wavelength fluorometer (National Agricultural University, Kiev, Ukraine) used for illumination of the predarkened leaves and control of fluorescence emission at two wavelengths at 690 and $735 \mathrm{~nm}$ [Posudin and Kozhemjako, 2001; 2002]

passes through a condensor which is formed by two quartz lens 2 and 3 , excitation filter 4 (FS-1-2 and SZS-24-2) and enters the sample 5 - green leaf. The registering of fluorescence radiation of the sample is realized at an angle of $30^{\circ}$ to the direction of excitation radiation in order to avoid the influence of it on the readout system. Lens 6 provides the focusing of fluorescence emission through filters $7(690 \mathrm{~nm})$ and $8(735 \mathrm{~nm})$ on the photomultiplier 9. A signal from the photomultiplier enters the input of the amplifier 10 (F136) and recorder 11 (KSP-4). The photomultriplier, amplifier and recorder are linked with the source of voltage 12.

\subsection{RESULTS OF DETECTION OF CHLOROPHYLL FLUORESCENCE KINETICS}

\subsubsection{Fluorescence Indices}

The following fluorescence indices were used for quantitative estimation of the changes of fluorescence parameters of the plant under stress conditions: maximum fluorescence $f_{m}$; steady-state fluorescence $f_{s}$; fluorescence decrease $f_{d}=f_{m}-f_{s}$; vitality index $R f d=f_{d} / f_{s}$, which is measured in the 690- and 735-nm region: $R f d(690) / R f d(735) ;$ ratios $f_{m} 690$ $/ f_{m} 735$ and $f_{s} 690 / f_{s} 735$, and the stress adaptation index $A_{p}=1-[\operatorname{Rfd}(735)$ $+1] /[\operatorname{Rfd}(690)+1]$. 


\subsubsection{Determination of Pigments}

Photosynthetic pigments [chlorophyll $(a+b)$ and total carotenoids $(x+c)$ ] were determined spectrophotometrically (UV2001 PC, Shimadzu, Duisburg, Germany) using the method of Prof. H.K. Lichtenthaler (1987). The pigments were extracted in 100\% acetone and determined due to extinction coefficients at 750, 661.6, 644.8, and $470 \mathrm{~nm}$.

The content of chlorophyll and carotenoids in the leaves of different levels of greening is presented in Table 8.8.

Table 8.8 Results of calculation of the pigment content (extract with 100\% Acetone) in leaves of Pisum sativum

\begin{tabular}{l|c|c|c|c}
\hline \multirow{2}{*}{ Variety of Plant } & \multicolumn{3}{|c}{ Output Per Area } \\
\cline { 2 - 5 } & $\begin{array}{c}\mathrm{Chl} \mathrm{a} \\
{\left[\mathrm{mg} / \mathrm{m}^{2}\right]}\end{array}$ & $\begin{array}{c}\mathrm{Ch} / \mathrm{b} \\
{\left[\mathrm{mg} / \mathrm{m}^{2}\right]}\end{array}$ & $\begin{array}{c}\text { Carotenoids } \\
x+c\left[\mathrm{mg} / \mathrm{m}^{2}\right]\end{array}$ & $\begin{array}{c}\text { Chlorophylls } \\
a+b\left[\mathrm{mg} / \mathrm{m}^{2}\right]\end{array}$ \\
\hline $\begin{array}{l}\text { 1. Determinantny, dark green, } \\
\text { 1 node }\end{array}$ & 248.05 & 83.82 & 63.63 & 331.87 \\
$\begin{array}{l}\text { 2. Determinantny, dark green, } \\
\text { 2 nodes } \\
\text { 3. Determinantny, light green, } \\
\text { 3 nodes }\end{array}$ & 228.59 & 76.16 & 59.05 & 304.75 \\
$\begin{array}{l}\text { 4. Bakara, dark green, } \\
\text { 1 node }\end{array}$ & 173.01 & 54.25 & 48.01 & 227.26 \\
\hline
\end{tabular}

The dark green leaves (sample N1) of the type Determinantny contained a higher pigment content than the green leaves (sample N2) and light green leaves (sample N3). The dark green leaves of two kinds, Determinantny and Bakara, of the same age and nodal position showed considerable difference in pigment content (samples N1 and N4).

The parameters of chlorophyll fluorescence induction kinetics such as maximum fluorescence $f_{m}$, steady-state fluorescence $f_{s^{\prime}}$ fluorescence decrease $f_{d}=f_{m}-f_{s}$ and vitality index $R f d^{\prime}$ (at $735 \mathrm{~nm}$ ), $R f d^{\prime \prime}$ (at $690 \mathrm{~nm}$ ), $f_{m} 690 / f_{m} 735$ and $f_{s} 690 / f_{s} 735$, and stress adaptation index $A_{p}$ of the samples N1-N4 with different pigment content are presented in Table 8.9.

It is shown that the $R f d$ values measured in the $690 \mathrm{~nm}$ region were higher than in the $735 \mathrm{~nm}$ region. The values of ratios $f_{m} 690 / f_{m} 735$ and $f_{s} 690 / f_{s} 735$ decreased during greening of the leaves. Increase in the vitality indices $R f d^{\prime}$ and $R f d^{\prime \prime}$ from light green to dark-green leaf was demonstrated. 
Spectroscopic Analysis of Agronomic Plants $\mathbf{1 7 1}$

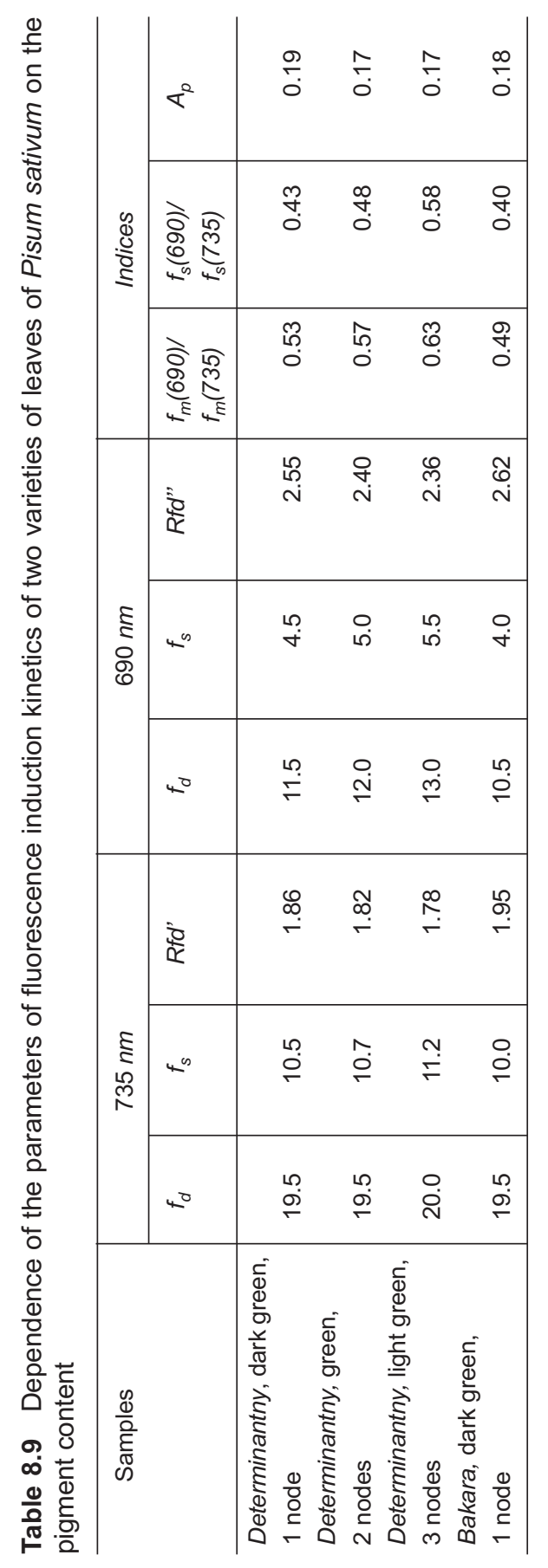


Both the contents of the main plant pigments, chlorophyll and total carotenoids, increased during greening. In the leaves with normal photosynthetic apparatus, the main part of the absorbed light energy is used for photochemical work to drive photosynthesis, and a small portion (about 2-5\%) of this energy is emitted as chlorophyll fluorescence. The dependence of the chlorophyll fluorescence parameters on the chlorophyll content can be explained in the following way [Lichtenthaler and Rinderle, 1988]: with increasing chlorophyll content, the short wavelength fluorescence becomes increasingly suppressed due to re-absorption of the emitted fluorescence by the chlorophyll which originates from the partial overlapping of the absorption and fluorescence emission spectra of the leaves between 640 and $740 \mathrm{~nm}$. The influence of re-absorption of the emitted fluorescence is less in leaves with low chlorophyll content; at such low chlorophyll content the values of the main fluorescence parameters, such as $f_{d}, f_{s}$ and $R f d$ exhibit higher values than in fully developed dark-green leaves. Besides, the changes in the pigment composition of the photosynthetic apparatus can also be taken into account. The difference of pigment content between two varieties of the same specie can be explained with the different levels of photosynthetic activity of these types.

\subsubsection{Effect of Leaf Nodal Position and Age}

The effect of nodal position of the leaves on chlorophyll fluorescence parameters is demonstrated in Table 8.10.

The values of fluorescence ratios $f_{m} 690 / f_{m} 735$ and $f_{s} 690 / f_{s} 735$, just as $R f d$ values declined with increasing leaf age from the youngest leaf of the first node to the older leaf (sixth node). The values of stress adaptation index $A_{p}$ do not demonstrate the significant changes in the limits of errors of measurements.

There was a considerable difference between the fluorescence parameters such as $f_{m} 690 / f_{m} 735$ and $f_{s} 690 / f_{s} 735$ of two kinds of green peas of the same age and nodal position.

The effect of nodal position and correspondingly the age of the leaves on chlorophyll fluorescence parameters can be explained by the formation of photosynthetic apparatus during leaf development. There are a number of factors which can be taken into account in the course of plant ontogeny [Sestak, 1985; Sestak and Siffel, 1988]: anatomical parameters, size, number and ultrastructure of chloroplasts, contents of pigments and pigment-protein complexes, composition and activities of enzymes of the carbon fixation pathways, rates of gas exchange and biomass production. These factors change from the time of leaf 


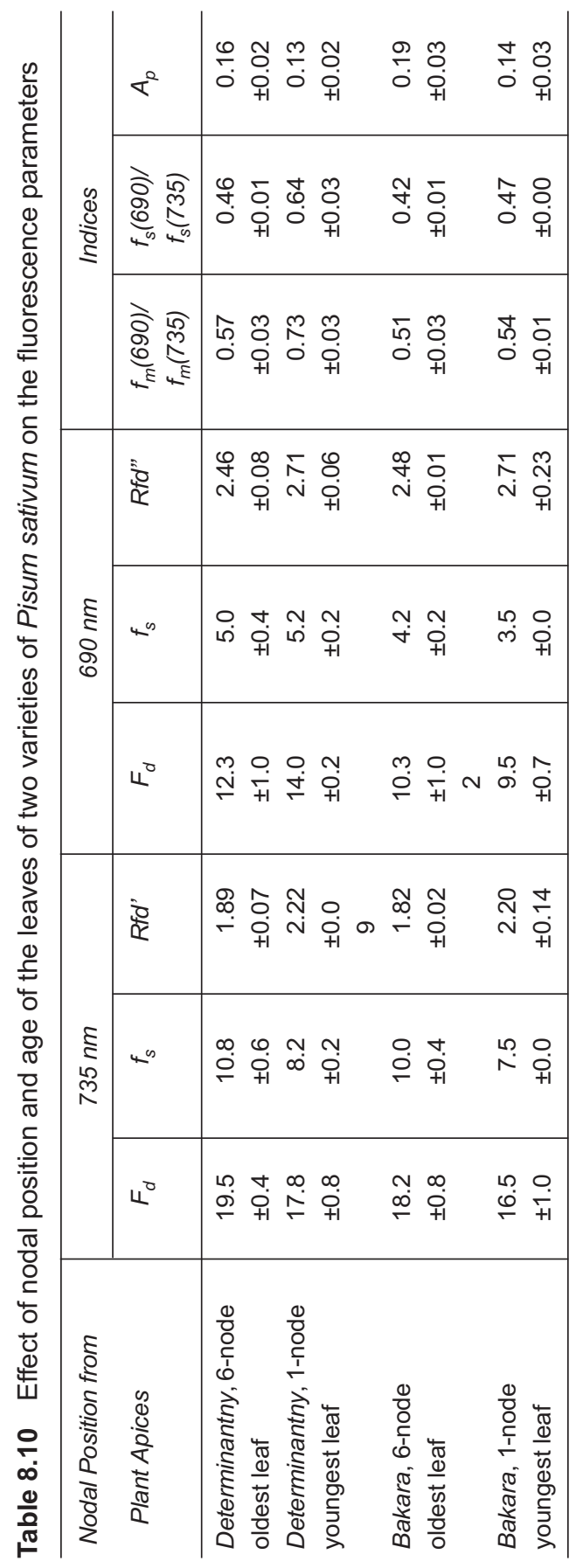




\section{Practical Spectroscopy in Agriculture and Food Science}

unfolding till the phase of leaf photosynthetic maturity and provoke the corresponding alterations of spectral properties of chloroplasts and leaves.

\subsubsection{Development of a Leaf}

The process of development of a leaf consists of two main phases - leaf synthesis and leaf degradation [Sestak, 1985]. The first phase is characterized by the ratios $f_{m} 690 / f_{m} 735<1$ and $f_{s} 690 / f_{s} 735<1$. Values above 1 are a clear sign of leaf degradation (Fig. 8.22). With increasing leaf age and during leaf senescence the $R f d$ values and $A_{p}$ values decrease (Table 8.11).

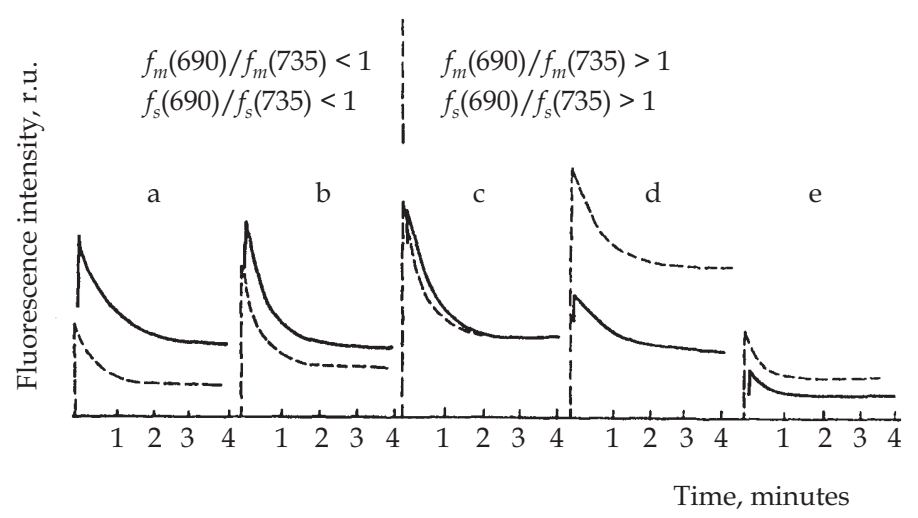

Fig. 8.22 Changes in the chlorophyll fluorescence induction kinetics of dark-adapted leaves of Pisum sativum (variety Determinantny) during leaf development $(a, b, c)$ and degradation $(d, e)$. $a$ - fully green, $b$ - light green, $c$ - green-yellowish, $d$ - yellow-greenish, e-yellow leaves; - $735 \mathrm{~nm},---690 \mathrm{~nm}$. Fluorescence excitation: He:Ne-laser, $632.8 \mathrm{~nm}$. The leaves were darkened for 15 minutes prior to illumination [Posudin, 1997, 2002]

The process of leaf development can provoke the changes of chlorophyll fluorescence also. The first stage of leaf development is accompanied by the increase of chlorophyll content. During leaf senescence, the photosynthetic quantum conversion decline, chlorophyll is broken down and the chlorophyll content per leaf area unit decreases. The process of leaf senescence is caused by factors such as progressive mineral eficiency, enhanced water and light stress, air pollution, virus infection and injury by insects [Lichtenthaler and Rinderle, 1988].

Changes in the fluorescence induction kinetics of the leaves under stress conditions (leaf abscission) can be explained by the hydration of the cytoplasma and the chloroplast stroma [Lichtenthaler and Rinderle, 


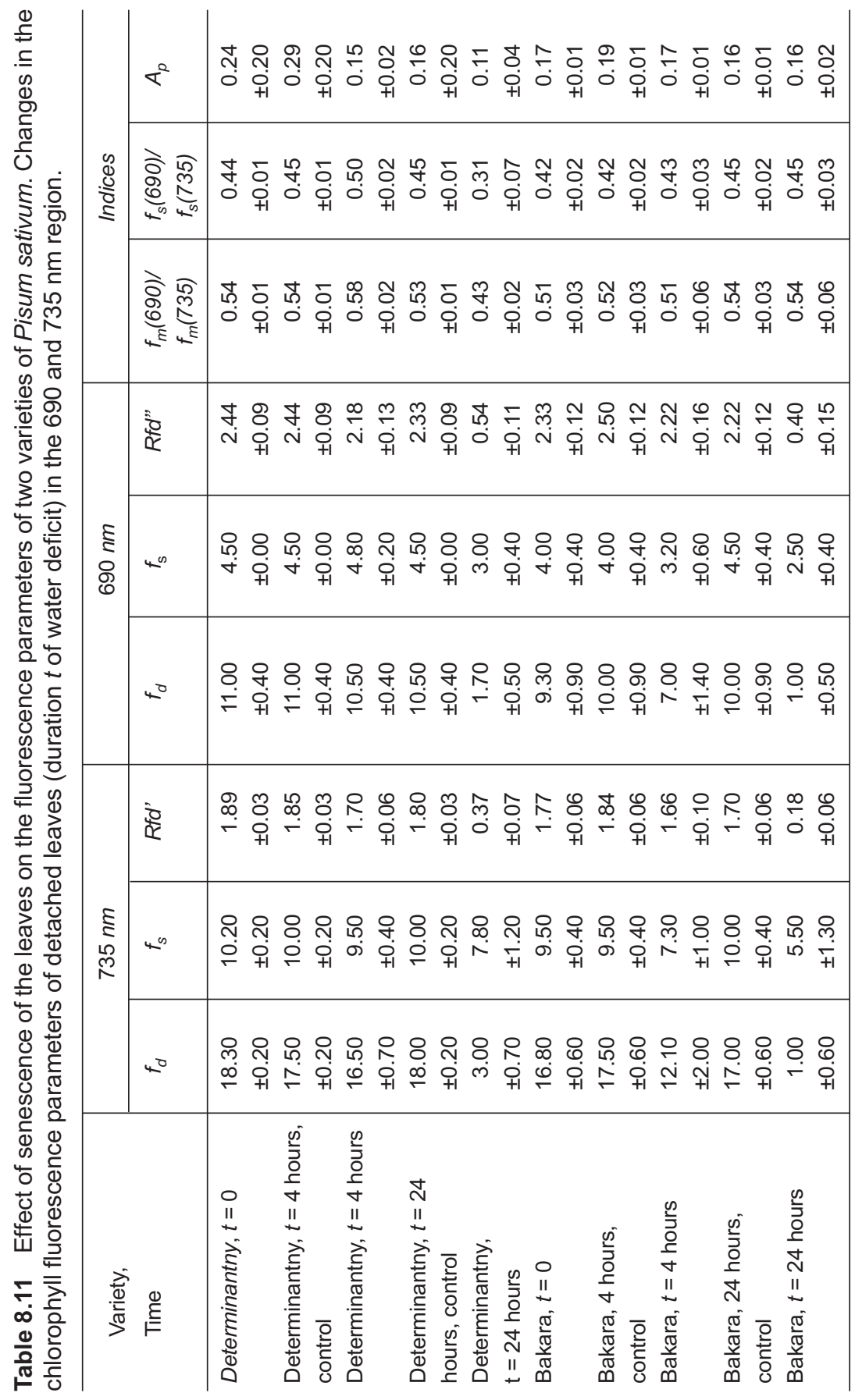




\section{Practical Spectroscopy in Agriculture and Food Science}

1988]. This water deficit provokes the desiccation of a cell and lowers the level of chlorophyll fluorescence emission because of reabsorption of the emitted fluorescence. Besides, the increasing dehydration of leaves causes the changes of the reflection properies of the leaves. Under stress conditions (leaf abscission) the leaf loses the photosynthetic function due to damage of the photosynthetic apparatus. It is shown [Lichtenthaler, 1990] that leaves with lower values of stress-daptation index $A_{p}$ suffer from stress earlier than those with a higher $A_{p}$-value.

\subsubsection{Upper and Lower Leaf Sides}

The results of chlorophyll fluorescence measurements from upper and lower leaf sides are presented in Table 8.12.

It is shown that the values for the ratios $f_{m} 690 / f_{m} 735$ and $f_{s} 690 / f_{s} 735$, $R f d$ values in $690 \mathrm{~nm}$ and $735 \mathrm{~nm}$ regions at the lower leaf side exceed the values at the upper leaf side for both species.

The dependence of chlorophyll fluorescence on the leaf side is related to the cross-sectional leaf structure. This structure in a typical dicotyledonous $\left(C_{3}\right)$ plant like a green pea (Pisum sativum) consists of the upper and lower epidermis which are separated by the mesophyll; the latter includes the palisade parenchyma cells and the spongy parenchyma cells (spongy mesophyll) [Gausman, 1985]. There is a difference in the structure and cell arrangement of the upper and lower sides of the leaves of $C_{3}$ plants - the upper side is characterized by more densely packed palisade parenchyma cells, and higher chlorophyll content and density than the lower one. That is why the re-absorption of emitted chlorophyll fluorescence of the lower side is much lower and fluorescence intensity is higher than in the upper side of the leaf. The fluorescence intensity near $690 \mathrm{~nm}$ is higher than in the $735 \mathrm{~nm}$ region because of the overlapping absorption and fluorescence spectra at 640$740 \mathrm{~nm}$ part of the spectrum.

\subsection{VECTOR METHOD OF FLUORESCENCE ANALYSIS OF PLANTS UNDER STRESS CONDITIONS}

\subsubsection{Introduction}

One of the main problems of biomonitoring is related to the search for effective test-objects and test-functions for the determination and estimation of the toxicity of different pollutants in aquatic media. It is possible to use bacteria, algae, protozoa, invertebrates, fish, and plants as test-objects during biomonitoring. Different test-functions such as biomass, growth-rate, photosynthesis, fluorescence, etc., are examined during toxicity testing of plants. 
Spectroscopic Analysis of Agronomic Plants

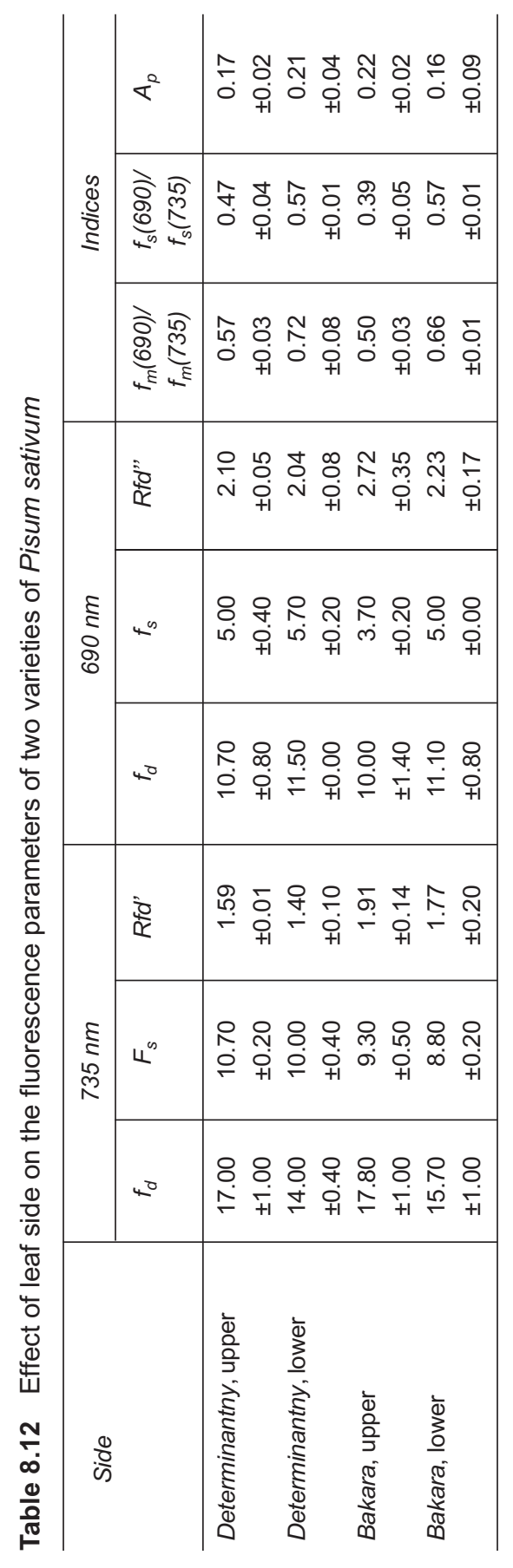




\section{Practical Spectroscopy in Agriculture and Food Science}

Nondestructive spectroscopic analysis of plants can be realized on the basis of two methodological approaches. The first one provides the examination of fluorescence emission spectra of green leaves which exhibit fluorescence maxima near 440-450 nm (blue region), at $690 \mathrm{~nm}$ and at $735 \mathrm{~nm}$ (red region). The ratio of the fluorescence intensities F690/ F735, which depends strongly on the chlorophyll content of the leaf, can be used as the fluorescence index that correlates with the rate of photosynthesis and the effect of different stress factors on photosynthetic activity of leaves.

The second approach is based on the detection of fluorescence kinetics of dark-adapted leaves (induction of fluorescence, Kautsky effect). Fluorescence kinetics reflects the sum total of processes which are linked with photosynthesis activity of a plant and also depends on the effect of different stress factors. The measurements of several chlorophyll fluorescence parameters such as vitality index $R f d=f_{d} / f_{s}$, particularly, $R f d(735)$ and $R f d(690)$, and stress adaptation index $A_{p}=1-[R f d(735)+1] /$ $[R f d(690)+1]$ provide useful information about healthy status of the leaf under stress conditions.

The vector method of quantitative estimation of stress factors on fluorescence parameters of chlorophyll was proposed for the first time by Posudin (1992). This method is based on excitation of chlorophyll fluorescence, simultaneous control of several fluorescence indices, and estimation of value and direction of the vector $P_{R}$, which is constructed in $N$-dimensional system of coordinates ( $N$ is a number of fluorescence indices which are recorded simultaneously); each axis of this system presents the ratio of fluorescence index under stress conditions and control fluorescence index. It is shown that the value and direction of the vector depend strongly on the status of the plant and the effect of different stress factors on it.

\subsubsection{Principle of Vector Method}

In the two-dimensional system of coordinates $(N=2)$, the value $r$ and the direction $\theta$ are defined as follows:

$$
\begin{aligned}
& r=\sqrt{\left(F_{1} / F_{1 c}\right)}+\left(F_{2} / F_{2 c}\right)^{2} \\
& \theta=\arctan \left[\left(F_{1} / F_{1 c}\right) /\left(F_{2} / F_{2 c}\right)\right]
\end{aligned}
$$

where $F_{i}=$ fluorescence index of the sample in stress conditions; $F_{c}=$ fluorescence index of control sample.

In the three-dimensional system of coordinates $(N=3)$, the value $r$ and the directions $\left(\theta_{1}\right.$ and $\left.\theta_{2}\right)$ are determined as follows: 


$$
\begin{aligned}
r & =\sqrt{ }\left(F_{1} / F_{1 c}\right)^{2}+\left(F_{2} / F_{2 c}\right)^{2}+\left(F_{3} / F_{3 c}\right)^{2} \\
\theta_{1} & \left.=\arccos \left[F_{1} / F_{1 c}\right) / r\right] \\
\theta_{2} & =\arctan \left[\left(F_{3} / F_{3 c}\right) /\left(F_{1} / F_{1 c}\right)\right]
\end{aligned}
$$

The value $r$ and the direction $\left(\theta_{1}, \theta_{2}\right.$ and $\left.\theta_{3}\right)$ of the vector $\vec{R}$ in the four-dimensional system of coordinates $(N=4)$ are determined from the following equations:

$$
\begin{aligned}
& r=\sqrt{ }\left(F_{1} / F_{1 c}\right)^{2}+\left(F_{2} / F_{2 c}\right)^{2}+\left(F_{3} / F_{3 c}\right)^{2}+\left(F_{4} / F_{4 c}\right)^{2} \quad \text { (Eqn. 8.6) } \\
& F_{1} / F_{1 c}=r \cos \theta_{1} \\
& F_{2} / F_{2 c}=r \sin \theta_{1} \cos \theta_{2} \\
& F_{3} / F_{3 c}=r \sin \theta_{1} \sin \theta_{2} \cos \theta_{3} \\
& F_{4} / F_{4 c}=r \sin \theta_{1} \sin \theta_{2} \sin \theta_{3}
\end{aligned}
$$

The $N$-dimensional system of coordinates can also be used, but in such situations $(N>4)$ the graphical description of vector $\vec{R}$ is impossible; its value and direction can only be tabulated.

\subsubsection{Application of Vector Method}

The concrete examples of application of vector method on the basis of fluorescence indices such as $\operatorname{Rfd}(690), \operatorname{Rfd}(735)$ and $A_{p}$ (method of fluorescence kinetics), and $F(690) / F(735)$ (method of spectrofluorometry) are detailed below:

\begin{tabular}{|c|c|c|c|c|}
\hline Colour & $\begin{array}{l}\operatorname{Rfd}(690) / \\
\operatorname{Rfd}(690)_{c}\end{array}$ & $\begin{array}{l}\operatorname{Rfd}(735)_{i} / \\
\operatorname{Rfd}(735)_{c}\end{array}$ & $r$ & $\theta,^{\circ}$ \\
\hline Dark green (control) & 1.00 & 1.00 & 1.41 & 45.00 \\
\hline Green & 0.70 & 0.80 & 1.06 & 41.20 \\
\hline Light green & 0.54 & 0.65 & 0.84 & 39.70 \\
\hline Yellow green & 0.30 & 0.37 & 0.48 & 39.00 \\
\hline
\end{tabular}

1. Registration of two fluorescence indices $R f d(690)$ and $R f d(735)$ of the leaves of green pea Pisum sativum of different chlorophyll content:

Value $r$ and direction $\theta$ of vector $\vec{R}$ in the two-dimensional system of coordinates that are determined by the formulae (8.1)-(8.2), are given in Table 8.13.

Table 8.13 Relative values of fluorescence indices, value $r$ and direction $\theta$ of vector $\vec{R}$ for leaf of green pea Pisum sativum of different chlorophyll content 
The dependence of value $r$ and direction $\theta$ of vector $\vec{R}$ in the twodimensional system of coordinates on the colour of leaves is presented in Fig. 8.23.

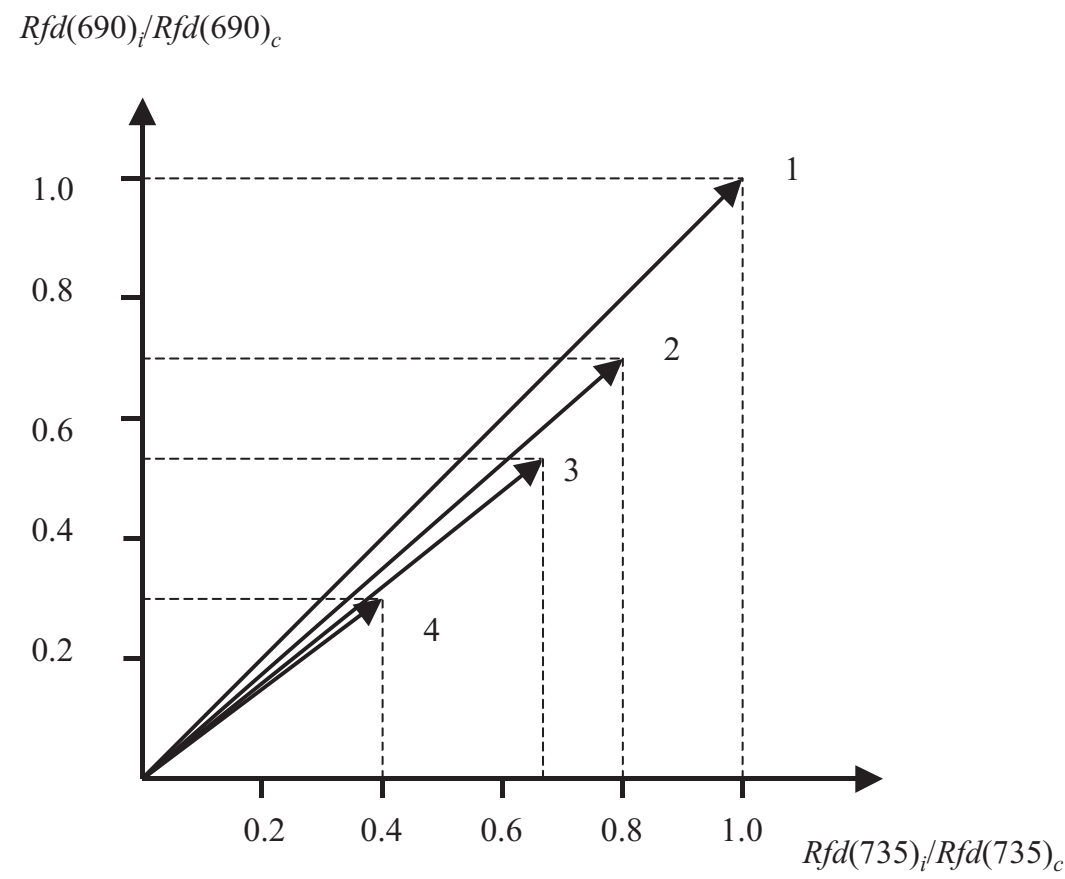

Fig. 8.23 Dependence of value $r$ and direction $\theta$ of vector $\vec{R}$ in the two-dimensional system of coordinates on the colour of leaves of green pea Pisum sativum: 1 - dark green leaf (control); 2 - green; 3 - light green; 4 - yellow green leaf

2. Registration of three fluorescence indices $R f d(690), R f d(735)$ and $A_{p}$ for the leaves of green pea Pisum sativum of different chlorophyll content:

Value $r$, direction $\left(\theta_{1}\right.$ and $\left.\theta_{2}\right)$ of vector $\vec{R}$ in the three-dimensional system of coordinates that are determined by the formulae (8.3)-(8.5), are given in Table 8.14.

The dependence of value $r$ and direction $\left(\theta_{1}\right.$ and $\left.\theta_{2}\right)$ of vector in the three-dimensional system of coordinates on the colour of leaves is presented in Fig. 8.24.

3. Registration of two fluorescence indices $R f d(690)$ and $R f d(735)$ of the leaves of green pea Pisum sativum under water deficit conditions: 
Table 8.14 Relative values of fluorescence indices, value $r$ and direction $\left(\theta_{1}\right.$ and $\theta_{2}$ ) of vector $\vec{R}$ in the three-dimensional system of coordinates for leaf of green pea Pisum sativum of different chlorophyll content

\begin{tabular}{|c|c|c|c|c|c|c|}
\hline Colour & $\begin{array}{l}R f d(690)_{i} / \\
\operatorname{Rfd}(690)_{c}\end{array}$ & $\begin{array}{l}R f d(735) f \\
\operatorname{Rfd}(735)_{c}\end{array}$ & $A_{p i} / A_{p c}$ & $r$ & $\theta_{1}^{\circ}$ & $\theta_{2}^{\circ}$ \\
\hline Dark green (control) & 1.00 & 1.00 & 1.00 & 1.73 & 54.68 & 45.00 \\
\hline Green & 0.70 & 0.80 & 0.54 & 1.19 & 53.96 & 37.64 \\
\hline Light green & 0.54 & 0.65 & 0.37 & 0.92 & 54.06 & 34.42 \\
\hline Yellow green & 0.30 & 0.37 & 0.20 & 0.52 & 54.76 & 33.69 \\
\hline
\end{tabular}

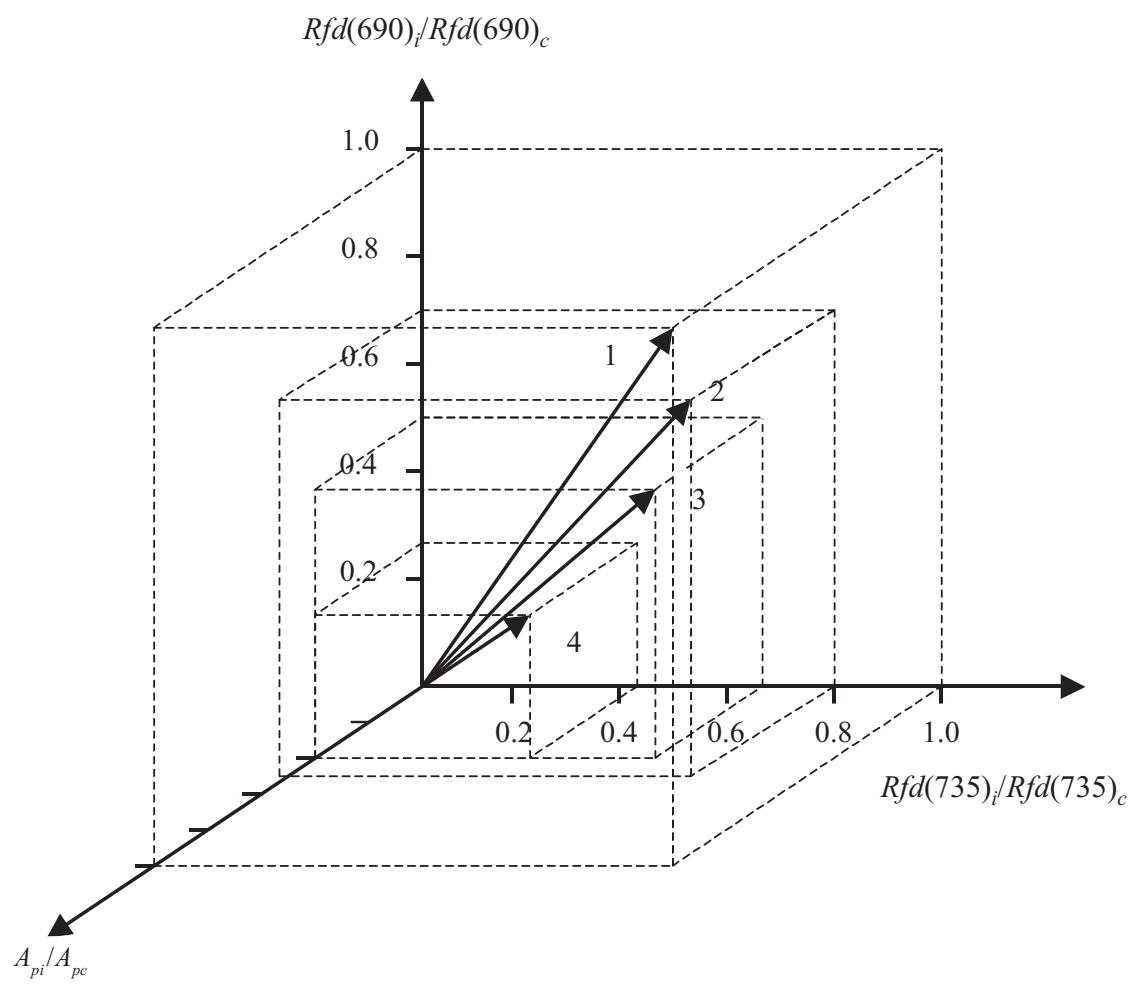

Fig. 8.24 Dependence of value $r$ and direction $\left(\theta_{1}\right.$ and $\left.\theta_{2}\right)$ of vector $\vec{R}$ in the threedimensional system of coordinates on the colour of leaves of green pea Pisum sativum: 1 - dark green leaf (control); 2 - green; 3 - light green; 4 - yellow green leaf

Value $r$ and direction $\theta$ of vector $\vec{R}$ in the two-dimensional system of coordinates that are determined by the formulae (8.1)-(8.2), are given in Table 8.15 . 
182 Practical Spectroscopy in Agriculture and Food Science

Table 8.15 Relative values of fluorescence indices, value $r$ and direction $\theta$ of vector $\vec{R}$ for leaf of green pea Pisum Sativum under water deficit conditions

\begin{tabular}{|c|c|c|c|c|}
\hline Water Deficit, Hours & $\begin{array}{l}\operatorname{Rfd}(690)_{i} / \\
\operatorname{Rfd}(690)_{c}\end{array}$ & $\begin{array}{l}\operatorname{Rfd}(735)_{i} / \\
\operatorname{Rfd}(735)_{c}\end{array}$ & $r$ & $\theta,^{\circ}$ \\
\hline$t=0$ (control) & 1.00 & 1.00 & 1.41 & 45.0 \\
\hline$t=1.5$ & 0.83 & 0.91 & 1.23 & 42.4 \\
\hline$t=4$ & 0.57 & 0.64 & 0.86 & 41.7 \\
\hline$t=24$ & 0.07 & 0.09 & 0.11 & 37.9 \\
\hline
\end{tabular}

The dependence of value $r$ and direction $\theta$ of vector $\vec{R}$ in the twodimensional system of coordinates on the water deficit of leaves is presented in Fig. 8.25.

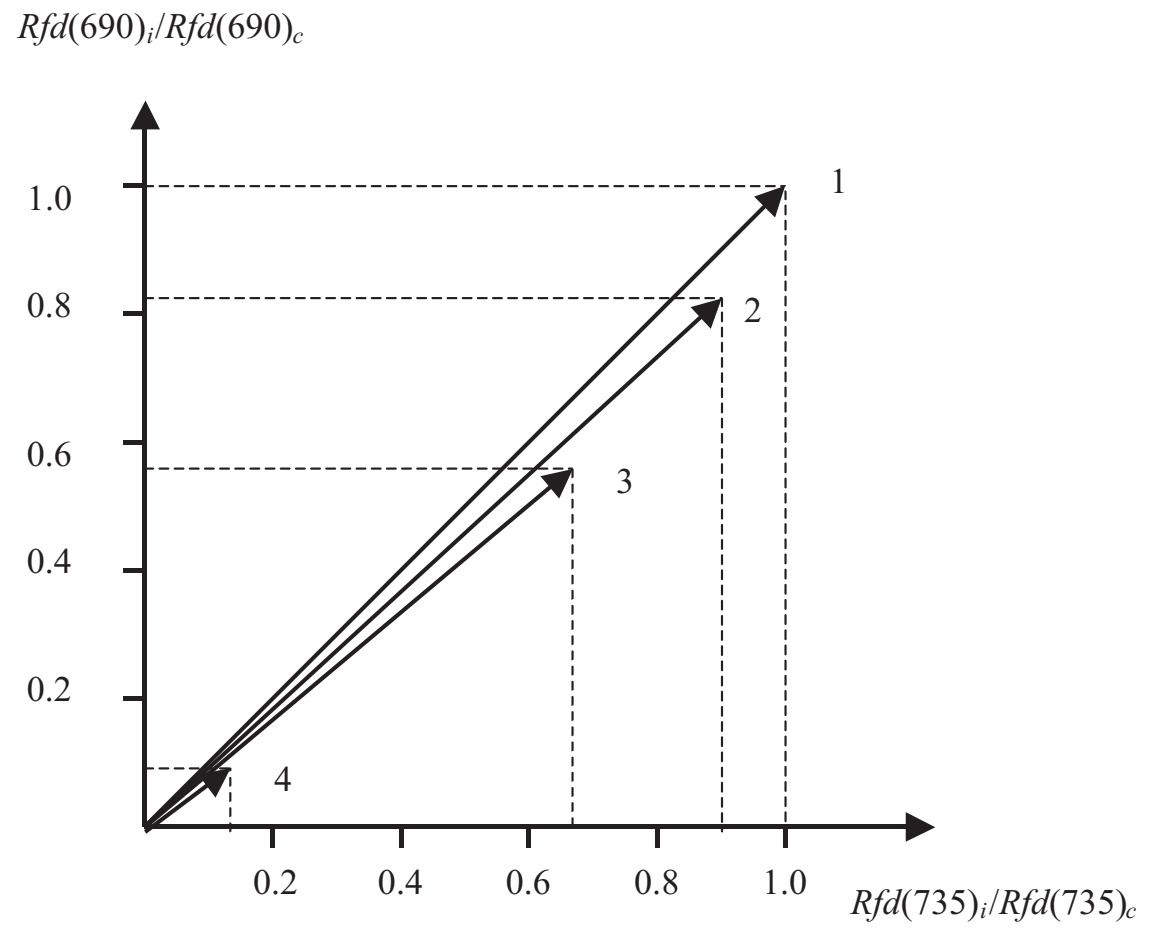

Fig. 8.25 Dependence of value $r$ and direction $\theta$ of vector $\vec{R}$ in the two-dimensional system of coordinates on the water deficit of leaves of green pea Pisum sativum: 1 control; 2 - after 1.5 hours of water deficit; 3 - after 4 hours; 4 - after 24 hours

4. Registration of three fluorescence indices $\operatorname{Rfd}(690), \operatorname{Rfd}(740)$ and $A_{p}$ for the leaves of green pea Pisum sativum under water deficit conditions: 
Value $r$, direction $\left(\theta_{1}\right.$ and $\left.\theta_{2}\right)$ of vector $\vec{R}$ in the three-dimensional system of coordinates that are determined by the formulae (8.3)-(8.5), for the leaves of green pea Pisum sativum with normal water supply and under water stress are given in Table 8.16.

Table 8.16 Relative values of fluorescence indices, value $r$ and direction $\left(\theta_{1}\right.$ and $\theta_{2}$ ) of vector $\vec{R}$ in the three-dimensional system of coordinates for leaves of green pea Pisum sativum with normal water supply and under water stress

\begin{tabular}{l|c|c|c|c|c|c}
\hline $\begin{array}{l}\text { Water Deficit, } \\
\text { Hours }\end{array}$ & $\begin{array}{r}R f d(690)_{i} / \\
\operatorname{Rfd}(690)_{c}\end{array}$ & $\begin{array}{c}R f d(740)_{i} / \\
\operatorname{Rfd}(740)_{c}\end{array}$ & $A_{p i} / A_{p c}$ & $r$ & $\theta_{1}{ }^{\circ}$ & $\theta_{2}{ }^{\circ}$ \\
\hline$t=0$ (control) & 1.00 & 1.00 & 1.00 & 1.73 & 54.69 & 45.0 \\
$t=2$ & 0.83 & 0.79 & 0.96 & 1.49 & 56.15 & 49.15 \\
$t=4$ & 0.47 & 0.42 & 0.92 & 1.12 & 65.19 & 62.94 \\
\hline
\end{tabular}

The dependence of value $r$ and direction $\left(\theta_{1}\right.$ and $\left.\theta_{2}\right)$ of vector in the three-dimensional system of coordinates on the water deficit of leaves of green pea Pisum sativum is presented in Fig. 8.26.

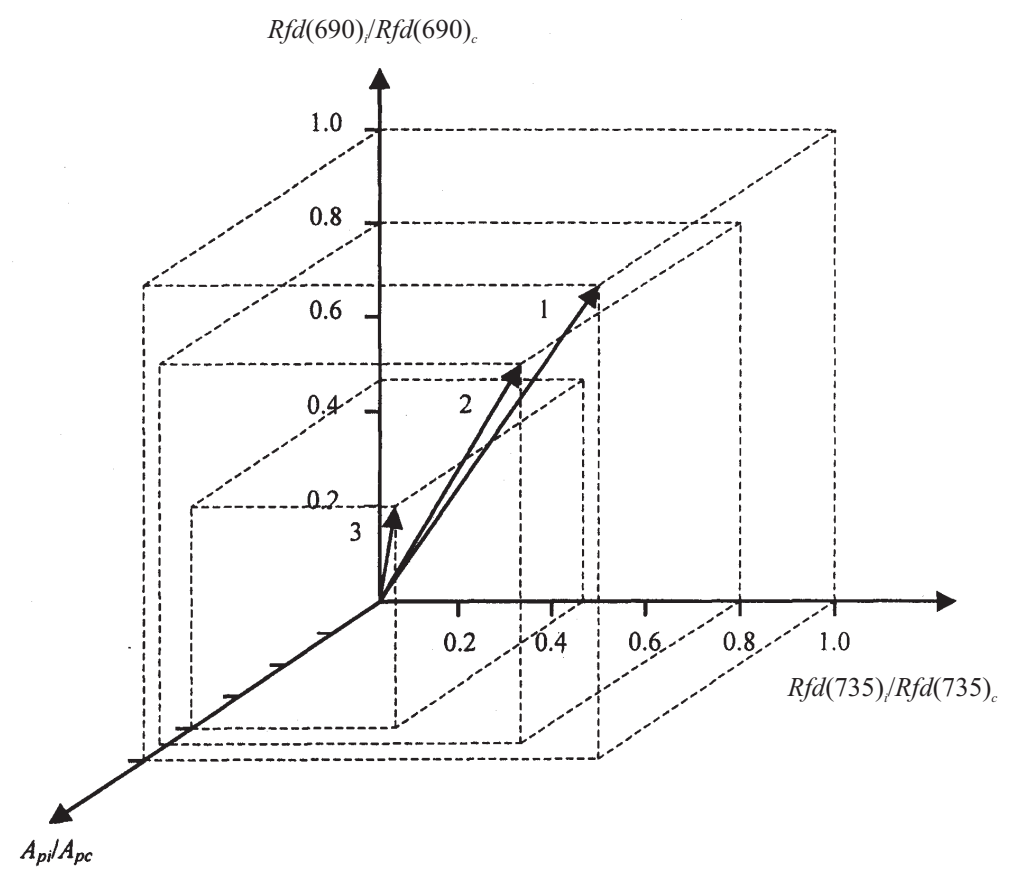

Fig. 8.26 Dependence of value $r$ and direction $\left(\theta_{1}\right.$ and $\left.\theta_{2}\right)$ of vector $\vec{R}$ in the threedimensional system of coordinates on the water deficit of leaves of green pea Pisum sativum: 1 - control; 2 - after 2 hours of water deficit; 3 - after 4 hours 


\section{Practical Spectroscopy in Agriculture and Food Science}

5. Registration of four fluorescence indices $R f d(690), \operatorname{Rfd}(740), A_{p}$ and F690/F735 for the leaves of green pea Pisum sativum under water deficit conditions:

Value $r$, direction $\left(\theta_{1}, \theta_{2}\right.$ and $\left.\theta_{3}\right)$ of vector in the four-dimensional system of coordinates that are determined by the formulae (8.6)-(8.10), for the leaves of green pea Pisum sativum with normal water supply and under water stress are given in Table 8.17.

\subsection{SUMMARY}

Method of laser spectrofluorometry in vivo of the leaves is rather promising for laboratory investigations of the effect of different agrochemical treatments and external physical factors; it is possible to control all the stages of growth and development of the plant, to study the effects of side and segment of the leaf, its nodal position, and the age. In some cases it is possible to achieve the species identification of the plants on the basis of the relevant spectral indices analysis.

The main perspective is the possible application of the results from single leaf investigation to the monitoring of agronomic plants at the canopy level and remote sensing of stressed agronomic fields on the basis of laser-induced fluorescence measurements.

Analysis of fluorescence induction kinetics of chlorophyll provides useful information about the process of the development of a leaf. Formation of internal leaf structure, increase of pigment content and intense fixation of photosynthetic activity accompany the first phase of leaf dvelopment. The next phase of leaf development is characterized by a prevalence of degradative processes such as declination of photosynthetic quantum conversion and decrease of chlorophyll content per leaf area unit. Both these processes provoke the relative changes of spectral properties of the leaf.

It has been found in these experiments that two varieties of the same species of green pea demonstrated certain differences between the fluorescence indices.

The measurements of several chlorophyll fluorescence parameters such as the fluorescence decrease $f_{d}$, maximum fluorescence $f_{m}$, steadystate fluorescence $f_{s}$, ratios $f_{m} 690 / f_{m} 735, f_{s} 690 / f_{s} 735$ and $F 690 / F 735$, vitality indices $R f d^{\prime}$ at $735 \mathrm{~nm}$ and $R f d^{\prime \prime}$ at $690 \mathrm{~nm}$ and stress adaptation index $A_{p}$ provide useful information about the development of a leaf and its healthy status under stress conditions. 
Spectroscopic Analysis of Agronomic Plants

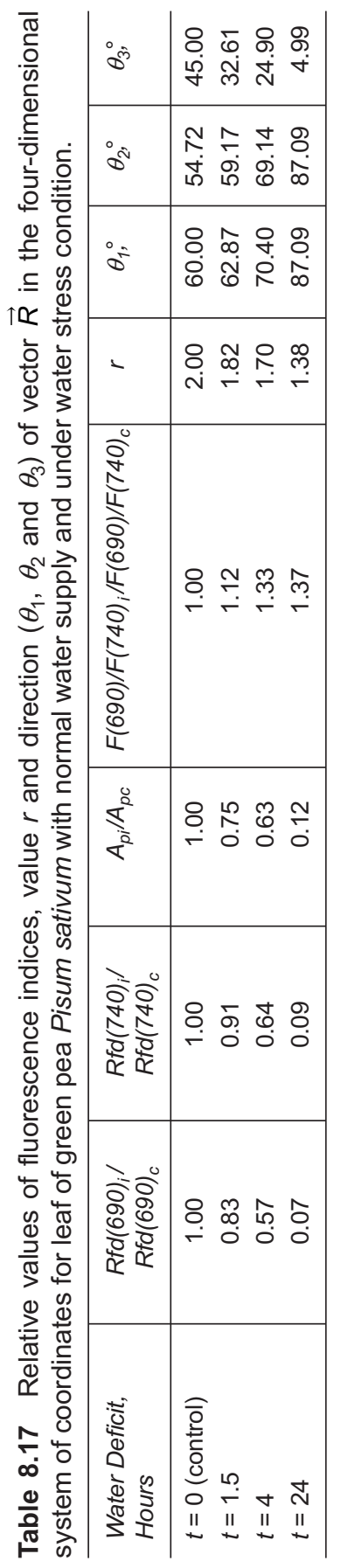




\section{Practical Spectroscopy in Agriculture and Food Science}

It is possible to use some of these fluorescence parameters as taxonomic criteria during investigation of the effect of age and nodal position of the leaves $\left(f_{m} 690 / f_{m} 735\right.$ and $\left.f_{s} 690 / f_{s} 735\right)$ and of leaf side $\left(f_{m} 690\right.$ $/ f_{m} 735, f_{s} 690 / f_{s} 735, R f d^{\prime}$ and $\left.R f d^{\prime \prime}\right)$.

The vector method of quantitative estimation of stress factors on fluorescence parameters of chlorophyll was applied to study the status of the plant and the effect of different stress factors on it. The simultaneous recording of several fluorescence parameters of chlorophyll and application of the vector method of biomonitoring can make it possible to realize the more precise quantitative estimation of these stress factors.

\subsection{REFERENCES}

Chappelle, E.W., Wood, F.M., McMurtrey, J.E. and Newcomb, W.W. 1984a. Laser-induced fluorescence of green plants. 1. A technique for remote detection of plant stress and species differentiation. Applied Optics 23: 134-138.

Chappelle, E.W., Wood, F.M., McMurtrey, J.E. and Newcomb, W.W. 1984b. Laser-induced fluorescence of green plants. 2. LIF caused by nutrient deficiencies in corn. Applied Optics 23: 139-142.

Chappelle, E.W., McMurtey, J.E. III and Kim, M.S. 1991. Identification of the pigment responsible for the blue fluorescence band in the laser-induced fluorescence (LIF) spectra of green vegetation plants, and the potential use of this band in remotely estimating rates of photosynthesis. Remote Sens. Environ. 36: 213-218.

Gausman, H.W. 1985. Plant Leaf Optical Properties in Visible and Near-Infrared Light. Graduate Studies Texas Tech University, 29: 78.

Gorodny, N.M., Povkhan, M.F., Posudin Yu.I. and Bykin, A.V. 1992. Fluorescence methods of control of the fertilizers efficiency. Agrochemistry. 4: 133-138.

Goulas, Y., Moya, I. and Schmuck, G. 1990. Time-resolved spectroscopy of the blue fluorescence of spinach leaves. Photosynth. Res. 25: 299-307.

Goulas, Y., Moya I., and Schmuck, G. 1991. Time-resolved spectroscopy of spinach leaves. In: Proc. 5th Intern. Colloq. Phys. Measurements and Signatures in Remote Sensing. Courchevel, pp. 715-718.

Hall, D. 1983. Photosynthesis. Moscow, Mir. p 132.

Ivanitskaya, S.A., and Posudin, Yu.I. 1989. Laser spectrofluoremetry of cereals. Biol. Sciences, Depon. VINITI, N5350-B89, p 10.

Ivanitskaya, S.A. and Posudin, Yu.I. 1989. Laser spectrofluorometry of vegetables. Biol. Sciences, Depon. VINITI, N5351-B89, p 9.

Kautsky, H. and Franck, U. 1943. Chlorophyll fluorescenz und kohleusäure assimilation. Biochemische Zeitschrift. 315: 139-232.

Kautsky, H. and Hirsch, A. 1931. Das Fluoreszenzverhalten gruner Pflanzen. Biochem. Z. 274: 422 .

Kautsky, H. and Hirsch, A. 1931. Neue Versuche zur Kohlenstoffassimilation. Naturwissenschaften. 19: 964.

Kays, S.J. and Paull, R.E. 2004. Postharvest Biology. Exon Press, Athens. p 568. 


\section{Spectroscopic Analysis of Agronomic Plants 187}

Kochubey, S.M. 1986. Organization of pigments of photosynthetic membranes as the basis of energy supply of photosynthesis. Kiev: Naukova Dumka, p 192.

Lang, M., Stober, F. and Lichtenthaler, H.K. 1991. Fluorescence emission spectra of plant leaves and plant constituents. Padiat. Environ. Biophys. 30: 333-347.

Lang, M., Siffel, P., Braunova, Z. and Lichtenthaler, H.K. 1992. Investigation of the Bluegreen Fluorescence Emission of Plant Leaves. Bot. Acta. 105: 435-440.

Lichtenthaler, H.K. 1987. Chlorophylls and carotenoids, the pigments of photosynthetic biomembranes. Methods Enzymol. 148: 350-382.

Lichtenthaler, H.K. and Rinderle, U. 1988. The role of chlorophyll fluorescence in the detection of stress conditions in plants. CRC Critical Reviews in Analytic Chemistry. 19: 29-85.

Lichtenthaler, H.K. 1988b. In vivo chlorophyll fluorescence as a tool for stress detection in plants. In: Application of Chlorophyll Fluorescence in Photosynthesis Research, Stress Physiology, Hydrobiology and Remote Sensing (Lichtenthaler H.K., ed.), Kluwer Academic Publishers, Dordrecht, pp. 129-142.

Lichtenthaler, H.K., Burkard, G., Kuhn, G. and Prenzel, U. 1981. Light-induced accumulation and stability of chlorophylls and chlorophyll proteins during chloroplast development in radish seedlings. Z. Naturforsh, 36: 421-430.

Lichtenthaler, H.K. 1990. Applications of chlorophyll fluorescence in stress physiology and remote sensing. In: Applications of Remote Sensing in Agriculture (Steven, M. and Clark, J.A., eds.) Butterworths Scientific Ltd., London, pp. 287-305.

Lichtenthaler, H.K., Lang, M. and Stober, F. 1991. Laser-induced blue and red chlorophyll fluorescence signatures of differently pigmented leaves. In: Proc. 5th Intern. Colloq. Phys. Measurements and Signatures in Remote Sensing. Courchevel. pp. 727-730.

Lichtenthaler, H.K., Stober, F. and Lang, M., 1993. Laser-induced fluorescence emission signatures and spectral fluorescence ratios of terrestrial vegetation. In: Intern. Geoscience and Remote Sensing Symposium, IGARSS' 93, Tokyo, Japan. V.III, pp. 1317-1320.

$\equiv$ , F.F. and Belyaeva, O.B. Characteristics of certain reactions and general scheme of biosynthesis of native forms of chlorophyll in etiolated plant leaves. Biochemistry (Russian) 36: 615-622.

Posudin, Yu.I. Instrument for measurement of fluorescence decay. Invention N 1263039 USSR, 1986.

Posudin Yu. I. 1988. Lasers in Agriculture. Science Publishers, Inc: Enfield, NH, USA. p 188.

Posudin Yu.I. 1992. Optical Methods of Investigation of Photobiological Reactions of Higher and Lower Plants. Thesis for a Doctor's degree. St. Petersburg: Agrophysical Institute, 1992. p 433.

Posudin, Yu.I. 1996. Methods of Optical and Laser Spectroscopy in Agriculture. Seminar Series of visiting Fulbright Scholar. University of Georgia, USA. January-April 1996.

Posudin, Yu.I. 1997. Spectroscopic monitoring of agronomic plants and canopies. Bull. Nat. Agr. Univ. 1: 14-21.

Posudin, Yu.I. and Kozhemjako, Y.V. 2001. Method of spectroscopic analysis of plants under stress conditions. Bull. Uzhgorod Nat. Univ. 10: 120-123.

Posudin, Yu.I. 2002. Fluorescence analysis of plants during development under stress conditions. Bull. Nat. Agr. Univ. 58: 42-48.

Posudin, Yu.I. and Kozhemjako, Y.V. 2002. Fluorometer for analysis of agronomic plants under stress conditions. Bull. Nat. Agr. Univ. 58: 39-41.

Posudin, Yu.I. and Kozhemyako, Y.V. 2003. Method of Analysis of Plants under Stress Conditions. Patent of Ukraine, 61148, N2001042240, 2003. 


\section{Practical Spectroscopy in Agriculture and Food Science}

Posudin, Yu.I. 2003. Vectorial method of fluorescence analysis of plants under stress conditions. Bull. Nat. Agr. Univ. 63: 45-49.

Posudin, Yu.I., Kucherov, A.P. and Olkhovskaya, G.P. 1991. Decomposition of spectral curves. Biol. Sciences, N254-B91, p 14.

Posudin, Yu.I., Ivonchik, P.N., Ivanitskaya, S.A. and Lilitskaya, G.G. 1992. Physical and biological methods of pesticides control. Bull. Agr. Science. 12: 36-38.

Sestak, Z. 1985. Photosynthesis during leaf development. Academia, Praha, Dr. W. Junk, Dordrecht-Boston-Lancaste.

Sestak, Z. and Siffel, P. 1988. Changes in chloroplast fluorescence during leaf development. In: Application of Chlorophyll Fluorescence in Photosynthesis Research, Stress Physiology, Hydrobiology and Remote Sensing (Lichtenthaler H.K., ed.), Kluwer Academic Publishers, Dordrecht, pp. 85-91.

Stober, F. and Lichtenthaler, H.K. 1992. Changes of the laser-induced blue, green and red fluorescence signatures during greening of etiolated leaves of wheat. J. Plant. Physiol. 140: 673-680.

Stober, F. and Lichtenthaler, H.K. 1993. Studies of the localization and spectral characteristics of the fluorescence emission of differently pigmented wheat leaves. Bot. Acta, 106: 365-370.

Stober, F., Lang, M. and Lichtenthaler, H.K. 1994. Blue, Green, and Red Fluorescence Emission Signatures of Green, Etiolated, and White Leaves. Remote Sens. Environ. 47: 65-71.

Subhash, N. and Mohanan, C.N. 1994. Laser-induced red chlorophyll fluorescence signatures as nutrient stress indicator in rice plants. Remote Sens. Environ. 47: 45-50. 


\section{About the Book}

This book deals with the practical applications of the methods of optical and laser spectroscopy to evaluation of state, content and quality of agricultural and food production, particularly, to:

- determination of content of fat, protein, and lactose in milk and dairy products;

- eggand eggshell quality evaluation, estimation of spoilage in eggs;

- laser control of bird feathering;

- honey quality evaluation and detection of adulteration;

- optical and laser spectroscopy of animal hair and use of spectral characteristics of the hair as genotypic indices of animal diversity;

- spectroscopic analysis of the status of agronomic plants under state conditions.

This book is meant for agricultural and food scientists, teachers, and students.

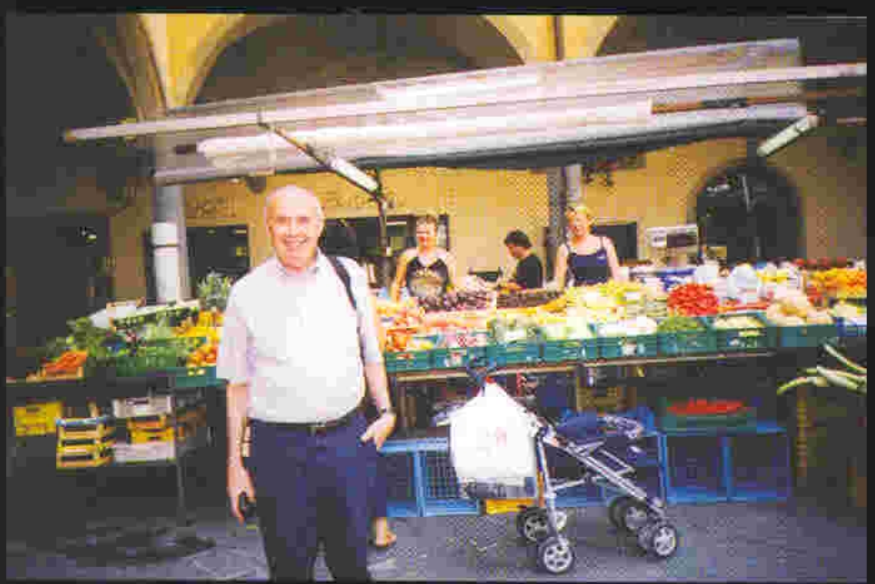

\section{About the Author}

Yuriy I. Posudin is a Professor at the National Agricultural University of Ukraine and Head of the Biophysics Department. He studied at the Kiev State University (Radiophysical University) 1964-1969, at the Institute of Radiotechnique and Electronics, Moscow (1972-1975), and at the Agrophysical Institute, St. Petersburg (1992).

His major scientific and educational interests include the applications of optical and laser spectroscopy to non-destructive quality evaluation of agricultural and food products. He delivered lectures at the University of Georgia, Athens, USA in 1996.

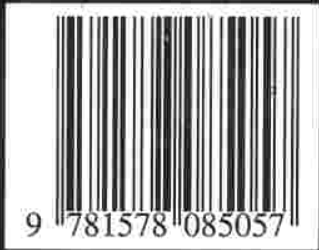

